\title{
OPTIMUM DESIGN OF COMPOSITE WING SPAR SUBJECTED TO FATIGUE LOADINGS
}

\author{
A Thesis \\ presented to \\ the Faculty of California Polytechnic State University, \\ San Luis Obispo
}

\author{
In Partial Fulfillment \\ of the Requirements for the Degree \\ Masters of Science in Aerospace Engineering
}

by

Juan Reuben Lazarin

June 2017 
(C) 2017

Juan Reuben Lazarin

ALL RIGHTS RESERVED 


\section{COMMITTEE MEMBERSHIP}

TITLE:

AUTHOR:

DATE SUBMITTED:

COMMITTEE CHAIR:

COMMITTEE MEMBER:

COMMITTEE MEMBER:

COMMITTEE MEMBER:
Optimum Design of Composite Wing Spar Subjected to Fatigue Loadings

Juan Reuben Lazarin

June 2017

Faysal Kolkailah, Ph.D., P.E Professor of Aerospace Engineering

Eltahry Elghandour, Ph.D.

Associate Professor of Mechanical Engineering

Eric Kasper, Ph.D., P.E.

Professor of Civil Engineering

Dianne DeTurris, Ph.D.

Professor of Aerospace Engineering 


\begin{abstract}
Optimum Design of Composite Wing Spar Subjected to Fatigue Loadings Juan Reuben Lazarin
\end{abstract}

Composites are now being incorporated into aircraft designs because of their high strength to weight ratio compared to traditional metal materials. Due to the complexity of the material, composite parts are presently being over designed to satisfy static and fatigue requirements. A greater understanding of composite fatigue behavior will allow for even greater weight savings leading to increased fuel economy. A critical part of an aircraft that is subjected to fatigue bending loads are its wings. The forces acting on the wings include its lift distribution, powerplant, and fuel which can be carried in the wing body. When in flight these forces repeatedly cause cyclic displacements which could ultimately lead to failure. It is important to design the wing spars which carry the bending loads, to be fatigue resistant so that damage or expensive inspections could be avoided.

Wing models were be made from composite materials with a NACA 0016 airfoil shape, chord length of 9.25", and a span of $15.25 "$. The C - channel spars were located at $22 \%$ and $72 \%$ of the chord. Strain gages on the wing model were used to measure strain at different locations. Static test were conducted on the specimens in order to validate a finite element analysis(FEA) model to be used for simulations. Overall, the strain measurements on the leading edge from two of the wings matched the model within $9 \%$ of the simulation results. Additional spar designs were then analyzed to determine the optimal one for static and fatigue bending loads. The wings were fatigue tested under displacement control at a test frequency. A model 8801 servo-hydraulic Instron machine and Wave Matrix software was used to fatigue the wings. After 100,000 cycles the test would be deemed a success and concluded. 


\section{ACKNOWLEDGMENTS}

I would like to thank my thesis advisors Dr. Faysal Kolkailah and Dr. Eltahry Elghandour for allowing me to work on such a challenging and interesting project. They were always there to support and motivate me throughout my time as a graduate student. Dr. Kolkailah was always there to offer advice on different aspects of the project from the simulation models to testing procedures. Dr. Elghandour's experience with composite materials and experimental testing made it possible for such a complex structure be manufactured and tested.

I want to also extend my gratitude to my committee members Dr. Dianne DeTurris and Dr. Eric Kasper for giving their suggestions throughout different phases of the project. They also spent much time from their schedules to review my work to insure that the highest quality is achieved. Dr. Eric Kasper helped to review various FEA models that were used in this project to insure accurate simulations. Dr. Dianne DeTurris provided assistance to insure that the process that I had taken throughout the project was methodical and well thought-out.

I would also like to thank our Aerospace Department Technician Cody Thompson, and assistants Kyle Rosenow and Greg Ritter for taking the time to machine various essential parts that were needed for this project. Sal Estévez, another assistant also provided great suggestions when initial fixture designs were being developed. Without this invaluable help these studies would not have been able to be conducted.

Many thoughts and advice were exchanged between my fellow graduate students which I am grateful for. They provided design ideas, manufacturing assistance, and support throughout this project. Mateja Andrejic helped early on in the design process and manufacturing of various part molds. Yanina Hallak provided her $\mathrm{CNC}$ router so that I could make aluminum parts. Sam Moss made multiple 
3D printed parts that were used for fast prototyping of fixture parts. Richard de Luna who was working on a similar project provided ideas for manufacturing aswell as testing. Jane Xiao was always there to provide feedback on different aspects of the project. Fritz Kaminski also gave much help by offering ideas on the project. Allen Capatina also helped by taking the time to machine aluminum metal ribs for this project.

Finally, I would like to thank my family including parents, grandparents, uncles and cousins for all the support and motivation throughout my schooling. I am grateful for my grandparents which would go out of their way to fill up a care package of food for me take to SLO after visiting. My parents have always pushed me to do the best in everything which allowed me to complete this challenging accomplishment. 


\section{TABLE OF CONTENTS}

Page

LIST OF TABLES ...................... . . . . . . . . .

LIST OF FIGURES . . . . . . . . . . . . . . . . . xii CHAPTER

1 INTRODUCTION $\ldots \ldots \ldots \ldots$.......... 1

1.1 Overview of Composites . . . . . . . . . . . . . 1

1.2 Types and Manufacturing . . . . . . . . . . . . . . . . . . 2

1.3 Wing Structure Background . . . . . . . . . . . . . . 6

1.4 Composite Fatigue Behavior . . . . . . . . . . . . . . . . 7

1.5 Previous Research on Fatigue Behavior of Composites . . . . . . . . 10

1.6 The Main Objective of this Study . . . . . . . . . . . . . 13

1.7 Scope of the Study . . . . . . . . . . . . . . . . . . 13

2 DESIGN METHODOLOGY OF WING AND FIXTURE $\quad$. . . 15

2.1 Wing Design Thought Process . . . . . . . . . . . . . 15

2.2 Wing Design . . . . . . . . . . . . . . . . . 18

2.3 Wing Fixture Design Process . . . . . . . . . . . . . 21

2.3.1 Fixture Design Considerations . . . . . . . . . . . 21

2.3.2 Initial Designs . . . . . . . . . . . . . . . . . 22

2.3.3 Final Fixture Design . . . . . . . . . . . . . . 27

3 WING MANUFACTURING . . . . . . . . . 35

3.1 Curing Cycle for Cycom and Hexcel Carbon Fiber . . . . . . . . . . 35

3.2 Wing Mold Design and Manufacturing . . . . . . . . . . . . 37

3.2.1 Female Mold . . . . . . . . . . . . . . . . . . 37

3.2 .2 Male Mold . . . . . . . . . . . . . . . . . . . . . 40 
3.2.3 Leading Edge Spar Mold . . . . . . . . . . . . . . . . 43

3.2.4 Trailing Edge Spar Mold . . . . . . . . . . . . . . . 44

3.3 Manufacturing of the Wing Skin . . . . . . . . . . . . . . 44

3.4 Manufacturing of the Spars . . . . . . . . . . . . 46

3.5 Manufacturing of the Ribs . . . . . . . . . . . . . . 48

3.6 Assembly of Wing Specimen . . . . . . . . . . . . . . . . 51

3.7 Strain Gage Mounting . . . . . . . . . . . . . . 57

4 TEST METHODOLOGY _........ 63

4.1 DAQ Selection Process . . . . . . . . . . . . . . 63

4.2 Model $8000 \mathrm{DAQ} \ldots \ldots \ldots$. . . . . . . . . . . . . . 64

4.2 .1 Connection and Setup . . . . . . . . . . . . 66

$4.2 .2 \quad$ Strain Smart Software _ . . . . . . . . . . . . 70

4.3 Material Properties . . . . . . . . . . . . . . . . . . 72

4.3 .1 Uni-axial Test Setup . . . . . . . . . . . . . . . . 72

$4.3 .2 \quad$ Fiber Volume Fraction . . . . . . . . . . . . . . . . 79

4.4 Validation for Experimental Static Test . . . . . . . . . . . 83

4.4.1 FEA Model Validation . . . . . . . . . . . . . 83

4.5 Experimental Static Test of the Wing . . . . . . . . . 90

4.6 Experimental Fatigue Test of the Wing $\ldots \ldots \ldots$

4.6.1 Experimental Fatigue Test Setup _. . . . . . . . . . 97

4.6.2 Fatigue Behavior at Different Test Frequency . . . . . . . 98

4.6.3 Strain Behavior under Static and Fatigue Loading _... 100

4.6.4 Fatigue Life Test . . . . . . . . . . . . . . . . . . 101

4.7 Static Test after Fatigue Test . . . . . . . . . . . . . . 102

5 EXPERIMENTAL RESULTS AND DISCUSSION $\ldots . . .103$

5.1 Material Property Results . . . . . . . . . . . . . . . 103 
5.1.1 Uni-axial Test Results . . . . . . . . . . . . . . . . . 103

5.1.2 Fiber Volume Fraction Results . . . . . . . . . . . . . . . 109

5.2 Experimental Static Test Results . . . . . . . . . . . . . . 111

5.2 .1 Validation Test Results . . . . . . . . . . . . . . . 112

5.2.2 Adjusted Fixture Constraint Results . . . . . . . . . . . . 115

5.2 .3 Wing Static Test Results . . . . . . . . . . . . . . 116

5.3 Fatigue Test Results . . . . . . . . . . . . . . . . . . . 122

5.3.1 Fatigue Behavior at Different Test Frequency Results . . . 122

5.3.2 Strain Behavior under Static and Fatigue Loading Results 124

5.3.3 Fatigue Life Test Results . . . . . . . . . . . . . . . . 126

5.4 Static Test after Fatigue Test Results _ . . . . . . . . . . . 129

6 FEA SETUP AND RESULT DISCUSSION $\ldots \ldots \ldots$

6.1 Selecting an FEA Solver . . . . . . . . . . . . . . 131

6.2 Model Validation FEA Results . . . . . . . . . . . . . . . . . . 132

6.3 Building the Wing Model . . . . . . . . . . . . . . . . 136

6.4 Static Simulation Results . . . . . . . . . . . . . . . . . . 140

6.5 Fatigue Simulation Results . . . . . . . . . . . . . . . . 149

7 RESULTS COMPARISON AND CONCLUSION $\ldots \ldots$

7.1 Model Validation Results Comparison . . . . . . . . . . . . . . 154

7.2 Static Simulation Results Comparison . . . . . . . . . . . 158

7.3 Conclusion . . . . . . . . . . . . . . . . . 163

7.4 Future Work . . . . . . . . . . . . . . . . . . . . . 164

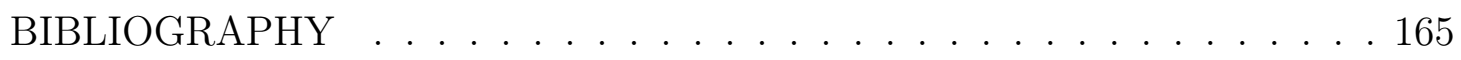

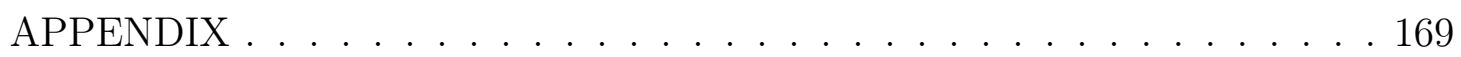




\section{LIST OF TABLES}

Table

5.1 Summary of aluminum sample material properties and percent errors from data sheet . . . . . . . . . . . . . . . . . . 104

5.2 Summary of Cycom tensile material properties and percent error from data sheet . . . . . . . . . . . . . . . . . 107

5.3 Summary of Hexcel tensile material properties and percent error from data sheet .................... 109

5.4 Measured Poisson's ratio for both composite materials used . . . . 109

5.5 Summary of the Hexcel volume fraction results . . . . . . . . . . . 110

5.6 Summary of the Cycom volume fraction results . . . . . . . . . 111

5.7 Comparison of experimental and analytical strain values of aluminum beam . . . . . . . . . . . . . . . . . . . 112

5.8 Test frequency study results . . . . . . . . . . . . . . . . . . . 123

5.9 Differences between test frequencies . . . . . . . . . . . . . . 123

6.1 Summary of the mesh densities used in the mesh convergence study 139

6.2 Summary of strain results from the 5 different spar designs simulated with the FEA model . . . . . . . . . . . . . . . . . . . . . . 148

7.1 Strain at strain gage location comparison for all three methods . . 155

7.2 Maximum displacement of aluminum beam comparison of FEA and analytical results . . . . . . . . . . . . . . 155 
7.3 Summary of strains along the Cycom Cantilever Beam . . . . . . 156

7.4 Summary of strains along the Hexcel Cantilever Beam . . . . . . 157 


\section{LIST OF FIGURES}

Figure

Page

1.1 Composition of materials that make up concrete . . . . . . . . . 2

1.2 Schematic of a wet layup and the materials used in the process [3] 3

1.3 Machines used to make two different types of composite parts . . 4

1.4 Diagram of a layup schedule with $0,-45,+45$, and $90^{\circ}$ plies . . . 5

1.5 Diagram of a honeycomb sandwich plate layup . . . . . . . . . . . 6

1.6 Parts on the Boeing 787 made out of composite materials are shown in blue $[6] \ldots \ldots \ldots \ldots$

1.7 Example of a stress life curve $(\mathrm{S}-\mathrm{N})$ along with its fatigue limit [9] 9

1.8 Example of a stress driven fatigue cycle and its amplitude definitions 10

1.9 Shaker used to apply a resonance on a test sample so that the modulus could be calculated $[7] \ldots \ldots$. . . . . . . . . . . . . 11

1.10 Visual of the warp and weft directions in a fabric [11] . . . . . . 11

1.11 DP-5/3 bending fatigue machine and its test diagram . . . . . . 12

2.1 Metal wing used in experimental analysis course at Cal Poly - San Luis Obispo . . . . . . . . . . . . . . . . . . . . 16

2.2 I beam spars enclosing on part mold f . . . . . . . . . . . . . 17

2.3 Different types of spars used in aircraft . . . . . . . . . . 18

2.4 Exploded view of the wing . . . . . . . . . . . . . 19

2.5 Spar design . . . . . . . . . . . . . . . . . . . 19

2.6 Spar dimensions . . . . . . . . . . . . . . . . 20 
2.7 Summary of wing design details . . . . . . . . . . . . 20

2.8 Initial hanging fixture design . . . . . . . . . . . . . . . 22

$2.950 \mathrm{lb}$ angle plate used to hold the final wing fixture $\ldots \ldots . . .23$

2.10 Picture of prototype wing failure at the root and spar connection 24

2.11 Solidworks model of the gripping fixture design $\ldots \ldots \ldots$

2.12 Locking key insert in final fixture part . . . . . . . . . 26

2.13 Prototyping front mount . . . . . . . . . . . . . . 27

2.14 Mounting fixture for the wing . . . . . . . . . . . 28

2.15 Fixture part dimensions . . . . . . . . . . . . . . . . 29

2.16 Root side of wing model mount . . . . . . . . . . . . . . . . 29

2.17 Exploded view of fixture $\ldots \ldots \ldots \ldots \ldots$

2.18 Fixture assembly . . . . . . . . . . . . . . . . 31

2.19 Wing tip mount assembly . . . . . . . . . . . . . . 32

2.20 Front mount dimensions . . . . . . . . . . . . . . . . 32

2.21 Wing tip mount $\ldots \ldots \ldots \ldots \ldots \ldots \ldots \ldots$

2.22 Final fatigue fixture assembly CAD model . . . . . . . . . . . 33

2.23 Final fatigue fixture assembly $\ldots \ldots \ldots \ldots \ldots$

3.1 Hexcel cure cycle . . . . . . . . . . . . . . . . . . 36

3.2 Cycom cure cycle . . . . . . . . . . . . . . . . . . 37

3.3 3D model of female mold . . . . . . . . . . . . . . . . . 38

3.4 Machining of female wing skin mold in progress . . . . . . . . . 39

3.5 Finished female mold ready to be used for skin layup . . . . . . 40

3.6 Bonding of aluminum sheet onto the porous foam mold . . . . . 41

3.7 Finished female mold ready to be used for skin layup . . . . . . 42

3.8 Cross-sectional dimensions of the leading edge spar $\ldots \ldots \ldots$

3.9 Cross-sectional dimensions of the trailing edge spar $\ldots \ldots \ldots 4$

3.10 Wing skin layup . . . . . . . . . . . . . . . . 45 
3.11 Wing skin layup . . . . . . . . . . . . . . 46

3.12 Spar layup . . . . . . . . . . . . . . . . . . . 47

3.13 Cured leading edge and trailing edge spars . . . . . . . . . 48

3.14 Applying templet on carbon fiber plate $\ldots \ldots \ldots$. . . . . 49

3.15 Cutting carbon fiber ribs $\ldots \ldots \ldots \ldots \ldots$

3.16 Finished rib part $\ldots \ldots \ldots \ldots \ldots \ldots \ldots \ldots$

3.17 Spar layup . . . . . . . . . . . . . . . . . . . 52

3.18 Method used to bond t-nuts onto ribs . . . . . . . . . . . 53

3.19 Bonding spars on to first rib . . . . . . . . . . . . . 54

3.20 Inner brackets bonded to provide pressure when bonding spars . 54

3.21 Bonding spars on to first rib . . . . . . . . . . 55

3.22 Internal structure goes into skin for bonding . . . . . . . . 56

3.23 Bonding wing skin onto internal structure $\ldots \ldots \ldots \ldots$

3.24 Finished wing model after sanding $\ldots \ldots \ldots \ldots$

3.25 Initial strain gage bonding steps . . . . . . . . . . . 58

3.26 Rosette strain gage ready for catalyst to be applied to it $\ldots . .59$

3.27 Uni-directional strain gage and terminal ready to be applied to bond

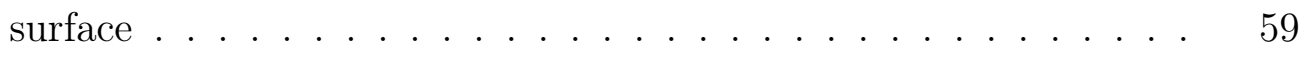

3.28 Bonded uni-directional strain gage and its terminal pad on specimen 60

3.29 Strain gage covered by tape to protect it from heat during soldering 61

3.30 Solder added to the terminals and strain gage tabs $\ldots \ldots \ldots 6$

3.31 Strain gages bonded and wired onto wing specimen $\ldots \ldots \ldots 6$

4.1 Model 8000 DAQ $[17] \ldots \ldots \ldots \ldots$

4.2 Close up of the RJ-45 cable and its 8 wires used for strain gage

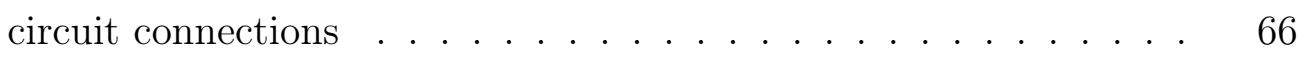

4.3 RJ- 45 circuit breakout $\ldots \ldots \ldots \ldots \ldots$

4.4 DAQ and computer for data collection $\ldots \ldots \ldots . \ldots 67$ 
4.5 Breakouts with 2 wire and 3 wire connections for the instron and strain gage signals respectively . . . . . . . . . . . . 68

4.6 Two of the connection types used with Model 8000 DAQ [20] . . . 69

4.7 Close up of uni directional strain gage used in experiments . . . . 70

4.8 Wheatstone bridge circuit showing the active strain gage and the shunt calibration resistor f. . . . . . . . . . . . 71

4.9 Two types of ways that Strain Smart software can display results $\quad 72$

4.10 Drawing of tensile test specimen . . . . . . . . . . . . 73

4.11 Abraded Tensile Specimens and Metal Tabs . . . . . . . . . . 74

4.12 Tensile specimens . . . . . . . . . . . . . . . . . 75

4.13 Specimen with strain gage . . . . . . . . . . . . . 75

4.14 Instron extensometer on tensile specimen . . . . . . . . . . . 77

4.15 Test specimens in Instron grips . . . . . . . . . . . . . . 78

4.16 Sample used in volume fraction testing per ASTM D2584 . . . . 80

4.17 Oven used to burn the epoxy out of the carbon sample for volume faction calculations . . . . . . . . . . . . . . . . . 81

4.18 Example of specimen after being cooked in the oven . . . . . . 82

4.19 Aluminum cantilever beam experimental test setup . . . . . . . . 84

4.20 Free body diagram of aluminum beam . . . . . . . . . 85

4.21 Equation used in analytical calculation of maximum displacement $\quad 86$

4.22 Close up of strain gages bonded onto cantilever beam specimen with wires ........................... 87

4.23 Carbon fiber cantilever testing . . . . . . . . . . . . . . 88

4.24 Matrix equations used in the analytical calculation . . . . . . 89

4.25 Free body diagram of cantilever beam problem . . . . . . . . . 89

4.26 Experimental test schematic . . . . . . . . . . . . . . . 91

4.27 Static testing of wing model . . . . . . . . . . . . . . . 91 
4.28 Strain gages on the wing model . . . . . . . . . . . . . . . . . 92

4.29 Dimensions between strain gages . . . . . . . . . . . . . . 93

4.30 Static test setup of wing . . . . . . . . . . . . . . . 94

4.31 Force and displacement test setup . . . . . . . . . . . . . 95

4.32 Test schematic . . . . . . . . . . . . . . . 96

4.33 Fatigue test setup . . . . . . . . . . . . . . . 97

4.34 Error at different fatigue test frequencies . . . . . . . . . . . . . 99

4.35 Data collection laptop collecting strain data during fatigue test . . 99

4.36 Fatigue test setup including Instron, wing, fixture, DAQ, latop . . 100

4.37 Position controled fatigue cycle . . . . . . . . . . . . . . 101

5.1 Stress stain curve of a failed composite tensile specimen . . . . . . 105

5.2 Extensometer and stain gage stress - strain curves . . . . . . 106

5.3 Stress stain curve of the Cycom specimens tested until failure . . 107

5.4 Stress stain curve of the Hexcel specimens tested till failure . . . . 108

5.5 Carbon fiber strain distribution along beam . . . . . . . . . . 114

5.6 Schematic of carbon fiber beam test . . . . . . . . . . . 115

5.7 Boundary condition filled in with epoxy and comparison results for

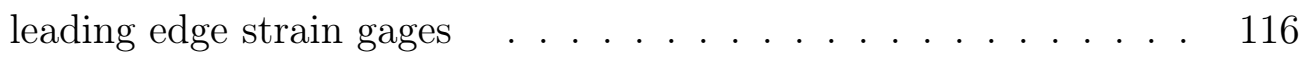

5.8 Comparison of carbon spacer and epoxy boundary condition adjust-

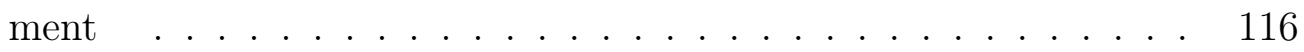

5.9 Strain values from the three wing models along the leading edge spar 117

5.10 Strain values from the three wing models along the trailing edge spar 118

5.11 Strain values from the three wing models along the chord-wise direction ................................ 119

5.12 Displacement curves for the wing with and without the mount . . 121

5.13 Comparison of the force and displacement study with and without

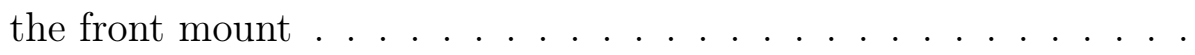


5.14 Displacement curves for the wing with and without the mount . .

5.15 Comparison of the force and displacement study with and without

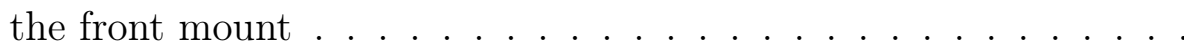

5.16 Fatigue force derivation from static and fatigue stain comparison . 125

5.17 Stain measurements during fatigue test . . . . . . . . . . . . 127

5.18 Displacement curves for the wing with and without the mount . . 130

5.19 Percent difference between initial and post fatigue testing of wing 3130

6.1 Strain distribution along the aluminum cantilever beam from FEA

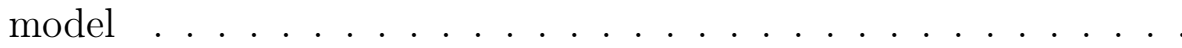

6.2 Cantilever beam model setup in ANSYS software . . . . . . . . . 134

6.3 Cantilever beam mesh and strain results . . . . . . . . . 135

6.4 Strains along the cantilever beams for both materials used in wing

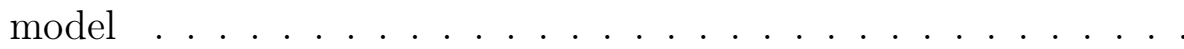

6.5 Strains along the cantilever beams for both materials used in wing

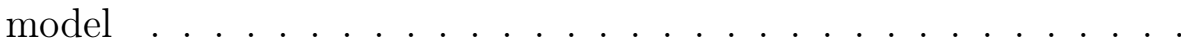

6.6 Shell model made in SolidWorks ready to be imported into ANSYS software ........................... 137

6.7 Setup of FEA wing model in ANSYS software . . . . . . . . . . . 138

6.8 Convergence of strain result due to mesh density . . . . . . . . . . 139

6.9 Comparison of mesh sizes used in the convergence study . . . . . 140

6.10 Strain distribution along the leading edge spar from the FEA sim-

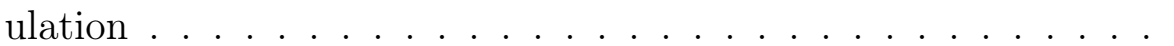

6.11 Strain result path for the leading edge spar results . . . . . . . . . 142

6.12 Strain distribution along the trailing edge spar for FEA and experimental data . . . . . . . . . . . . . . . . . 143

6.13 Strain result path for the trailing edge spar results . . . . . . . . . 143 
6.14 Strain distribution in the chord-wise direction for FEA and experimental data . . . . . . . . . . . . . . . . . . 144

6.15 Strain result path for the chord-wise results . . . . . . . . . . . 144

6.16 Two additional spar designs that utilized a one vertical web . . . . 145

6.17 Two additional spar designs that utilized a two vertical webs . . . 146

6.18 Strain distribution along the leading edge for each spar design simulated .......................... 147

6.19 Stress life curve model applied to both carbon fiber materials used in this paper $[38] \ldots \ldots \ldots \ldots \ldots$. . . . . . . . . . . . . . 149

6.20 Location where most of the spar designs showed initial failure . . 150

6.21 Location where the Z - spar design showed initial failure . . . . . 151

6.22 Stress life curves of each spar design . . . . . . . . . . . . . . 152

7.1 Strain distribution comparison along the aluminum cantilever beam 155

7.2 Strains along the cantilever beams for both materials used in wing model ...................... 157

7.3 Strain distribution comparison along the leading edge spar . . . . 159

7.4 Strain distribution along the trailing edge spar for FEA and experimental data . . . . . . . . . . . . . . . . . . . 160

7.5 Strain distribution in the chord-wise direction for FEA and experimental data . . . . . . . . . . . . . . . . . . 162 
Chapter 1

INTRODUCTION

\subsection{Overview of Composites}

Composites are made up of multiple materials that when combined offer great strength, lightweight, and customizable properties. First examples of this was Egyptian settlers using straw in mud to reinforce pottery or boat structures. In these early times, the fiber, which is the straw, and the matrix, in this case the mud, were derived from natural resources. These sources would be plants or even animals, which were readily available.

Not until the 1900's when plastics were invented did the modern era of composites start. These new synthetic resins were a lot stronger than the nature based binders that were used before, although the resin alone does not make the composite strong due to its brittle characteristics. In 1935, Owens Corning invented the first glass fiber, and when combined with synthetic resins, provided a strong and lightweight structure. This resembles the materials used today where a fiber is encased in a matrix. The fiber in the material has exceptional strength in tension, although by itself, it would not have any support. When the resin is added, its purpose is to transfer the load between the fibers, making a light and strong material together.

In WWII, lightweight applications such as aircraft, took composite materials from an idea into actual production. To this day, these materials are widely used in the aerospace field. In the 1970's, composites only improved due to even stronger 
resin and fibers. At this time, DuPont invented the aramid fiber, which is highly resistant to impacts, and therefore commonly used in bullet proof armor. Carbon fiber was also invented, which was even stronger than the other fibers, and is now replacing metal as the preferred material in many applications.[1]

\subsection{Types and Manufacturing}

One of the simplest composites that we encounter every day is concrete. It is classified as a composite, since it is made up of sand or aggregate (crushed stone) and incorporated together with a type of cement. In Fig.( 1.1) the constituents of the concrete can be seen. This material is inexpensive and can be used in many applications where weight is not an issue such as in foundations, buildings, and walls. Since airplanes must be lightweight to fly through the air, this is not an ideal material for the application.[2]

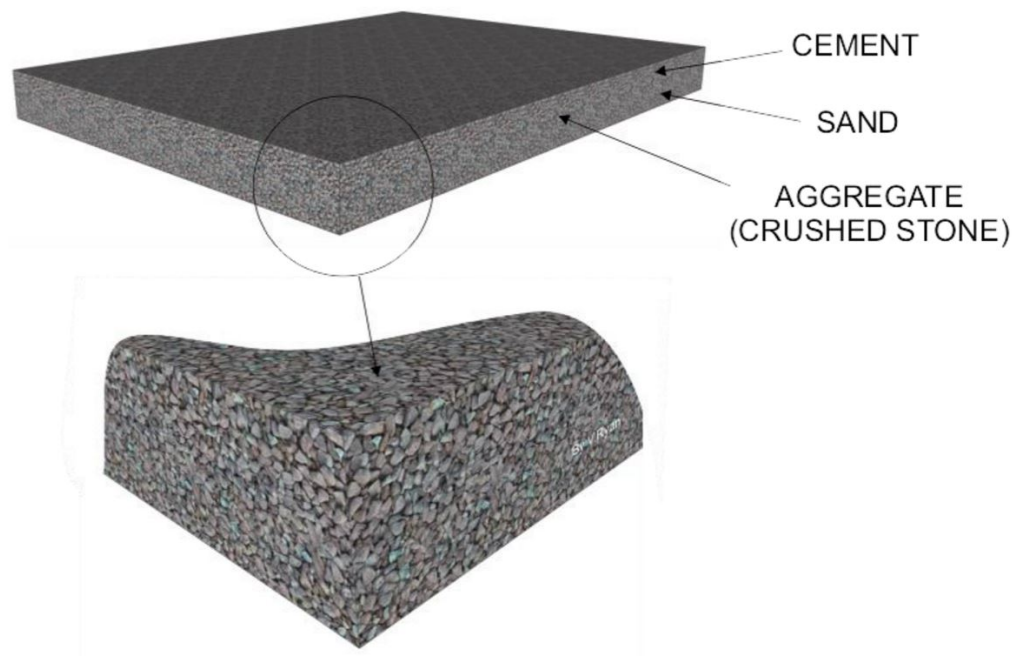

Figure 1.1: Composition of materials that make up concrete

Another form of composites is a dry fabric mat made of either uni-directional fibers or woven fibers. Weaving the fibers in different directions provides strength in those directions since the resin lacks strength for tensile forces. To make a part, the dry fabric is laid on top of a mold and an epoxy is poured on it and manually 
infused in a process called a wet layup. In Fig.( 1.2) a diagram of a wet layup is shown with the composite material shown in black. Temperature is then added to the part to expedite the curing process, and pressure is added in order to remove excess epoxy and air bubbles from the part. Air bubbles in the material would be voids that greatly reduce the strength of the material. The advantages of this method are that the fiber and the resin can be stored at room temperature for an extended period of time. This method is messy and requires careful measurements and procedures to yield an optimum fiber - matrix ratio. Even with a skilled technician, it can be difficult to yield a consistent ratio. Due to this inconsistency, it does not provide the confidence in the materials properties that is needed for safety concerns in some applications.

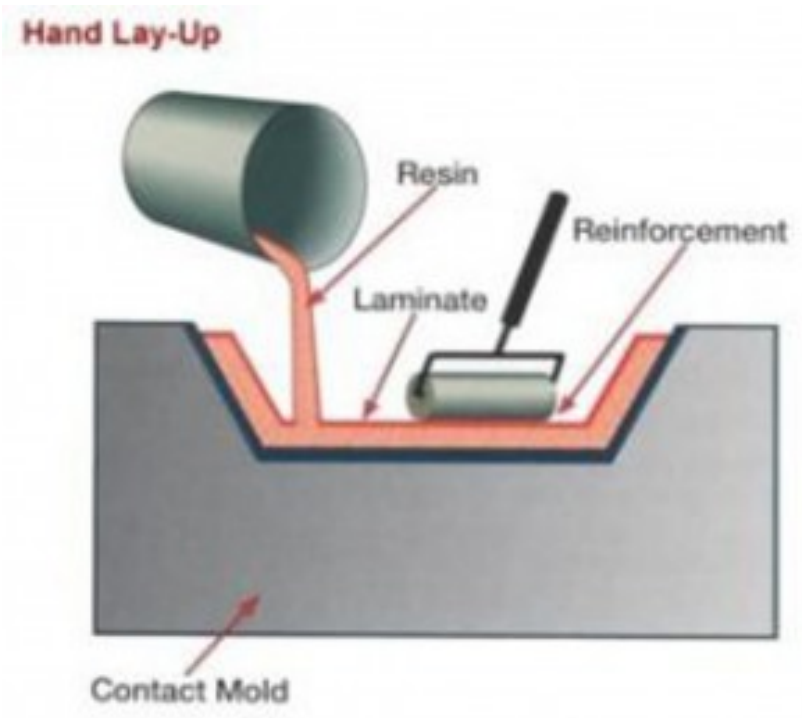

Figure 1.2: Schematic of a wet layup and the materials used in the process [3]

In order to have greater control over the fiber - matrix ratio, pre-impregnated composite fabrics were invented. In these materials, epoxy is introduced into dry fibers by the manufacturer. The resin is taken to a stage B level of cure making it easier to handle at room temperature. Since the epoxy is active in these materials, the rolls of composite need to be stored in a freezer below $0^{\circ} \mathrm{F}$ to stop the curing process. They are also sold in sealed bags to protect it from moisture. When a part 
is made, the material is laid into a mold and then vacuum bagged similarly as in the wet layup method. The temperatures required for pre-impregnated composites to be cured are higher, which can be applied by a heated press or an autoclave. Both of these items can be seen in Fig.( 1.3) below. All of the fiber and fabrics types can be found as pre-impregnated material. This type of composite is ideal for applications that require consistent material properties.

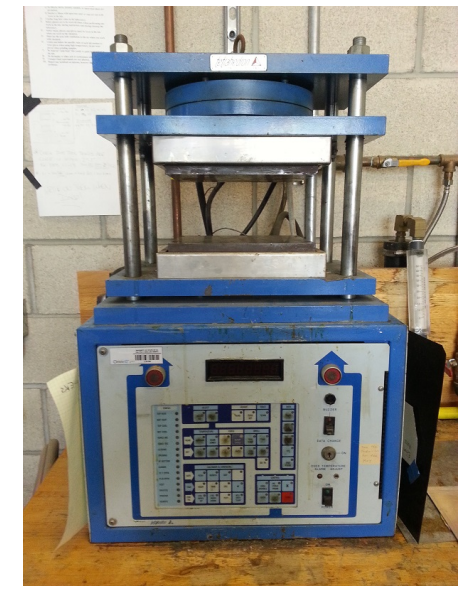

(a) Heated press to make composite laminates

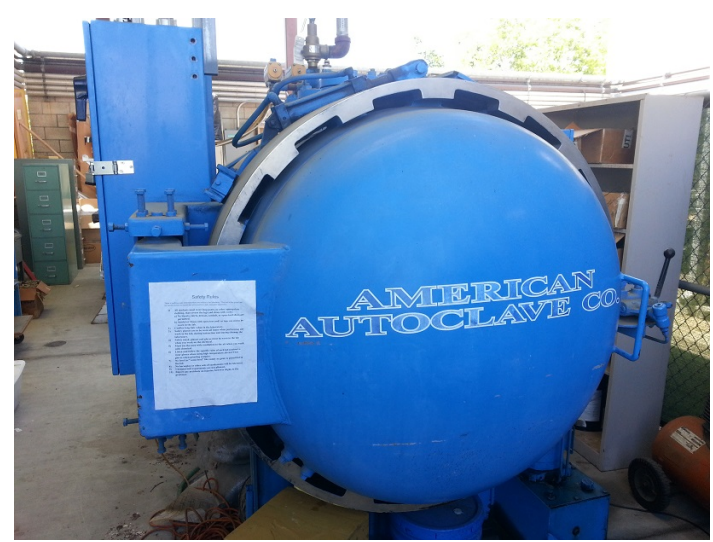

(b) Autoclave to make composite parts with complex geometries

Figure 1.3: Machines used to make two different types of composite parts

When multiple layers of a fabric are stacked to a certain thickness and cured together, it is considered a laminate. The plies of fabric in the laminate can be orientated in different directions for different applications. Since the fiber direction is most resistant to axial loads, it is beneficial to align it accordingly. Common angles used are $0^{\circ}, 45^{\circ}$, and $90^{\circ}$, where $0^{\circ}$ is in the direction of the axial load. The $45^{\circ}$ plies are best for resisting shear loads, where $90^{\circ}$ is beneficial when perpendicular loads are expected. In Fig.( 1.4) an example layup can be seen where different orientations are used to build a laminate. Having this ability to change the ply orientations allows for a customized layup for the application, effectively creating a new material.[4] 


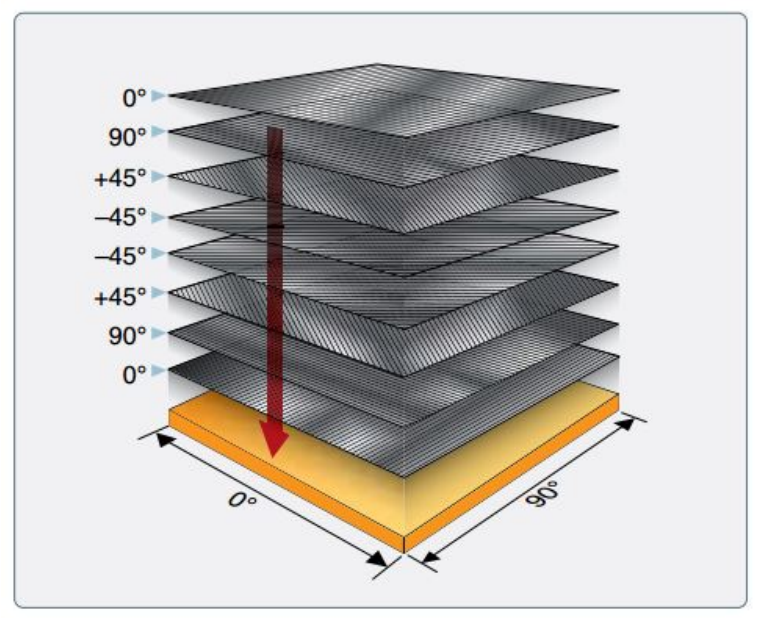

Figure 1.4: Diagram of a layup schedule with $0,-45,+45$, and $90^{\circ}$ plies

Another form of composite structures are sandwich panels, which are made up of two laminates with a thick lightweight core bonded between them. Foam and honeycomb materials are commonly used in sandwich structures to increase its stiffness. It does this by supporting the face sheets from buckling and out of plane forces. Honeycomb is a material made up multiple hexagon cells, making it light weight. Sandwich structures also have high bending stiffness at a low weight compared to having a pure carbon laminate of the same thickness. To layup a sandwich plate, carbon fiber is layered on either side of the core. The layup is then cured with heat and pressure in a press or autoclave. An example of the layup can be seen in Fig.( 1.5). Foam cores are cheaper alternatives to honeycomb, and are used on home built aircraft, but not in commercial applications, since it is heavier and not as strong.[4] 


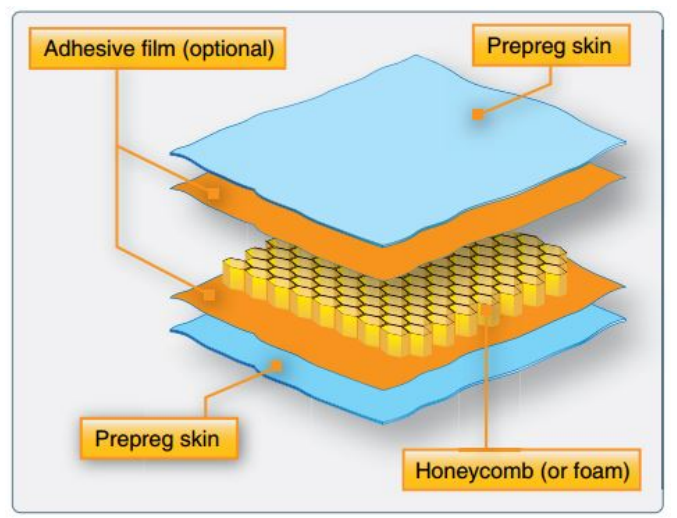

Figure 1.5: Diagram of a honeycomb sandwich plate layup

\subsection{Wing Structure Background}

An aircraft's wings are its main source of lift which allow it to fly through the air. In the process of doing this, the wings undergo different types of loads, but primarily bending. Even in steady level flight the wings undergo repeated deflections throughout its flight and service life. For this reason, the wing needs a structure that can resist considerable bending loads as well as fatigue loadings.

The wing's main resistance to bending loads is its spars, which are essentially beams that run from the body of the aircraft to the wing tip. The shape of the spars is designed to be stiff as well as lightweight. For this reason, shapes such as I - beams, C - channels, and box beams are used. These types of cross-sections maximize the moment of inertia by increasing the height rather than its width. Since their webs and flanges are made by thin sections these beams are made lightweight, increasing the airplanes fuel efficiency. Most commercial aircraft are designed to have two spars; one located closer to the nose of the airfoil, and another near the trailing edge.

The spars alone do not effectively resist all loads, such as in the case of torsion. Torques on the wing can come from control surfaces or disturbances in flight. In order to resist these types of loads, ribs are placed perpendicular to the spars. 
Multiple ribs are placed along the wing at equal distances. By creating a load path between the two spars, it minimizes the twist on the wing. The ribs effectively create a box shaped structure which has better torsional stiffness. Although, where these ribs connect to the spars tends to be a critical area for damage. These joints must be designed not only for static loadings, but also for fatigue scenarios. Recently, the A380 designed by Air Bus had a fatigue problem at these locations. This not only is a safely concern, but it can also increase repair and inspections cost, since they must be done more frequently.[5]

The last critical wing part is the wing skin, which is shaped as an airfoil so that it can produce lift. It is made of a thin sheet and bonded to the outside of the internal structure. The skin is thinner compared to the internal structure, although it does assist the spars and ribs by transferring loads between them.[4]

These parts in the past have been made of natural and metallic materials such as wood and aluminum respectively. A drive to have the lightest structure possible has given rise to composite materials being used. Since composite materials are relatively new, they need to be fully understood as metallic materials are now. One area where there is a need for greater understanding is composites fatigue behavior.

\subsection{Composite Fatigue Behavior}

The Boeing 787 was designed with more than $50 \%$ composites in its airframe, because it was driven by fuel efficiency, lightweight and high stiffness structures[6]. With the increasing use of composites in commercial aircraft, it is important to have a greater understanding of its fatigue behavior.[7] Composite materials are known to have great fatigue resistance, but there is much uncertainty when dealing with the fatigue of a large scale structure [6]. This makes certification challenging

because of the lack of confidence due to the lack of testing on assemblies. Fig.(1.6) 
shows a color plot of different materials that the Boeing 787 aircraft is made out of. On this aircraft, $50 \%$ of it is made out of composite materials.

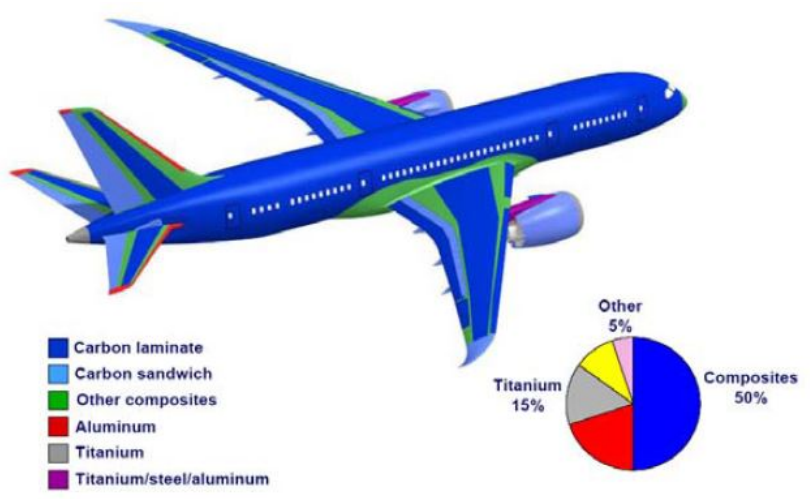

Figure 1.6: Parts on the Boeing 787 made out of composite materials are shown in blue [6]

Another hindrance that the limited fatigue testing on assemblies has caused is higher allowable margins needed for composite parts. Presently, in order to avoid complications, allowable strains for these parts are held to low magnitudes, although this limits the weight savings that could have been achieved by using the composite material. In the future, to further optimize aircraft, the allowables could be increased, but this would mean that the fatigue behavior would need to be researched extensively for parts and assemblies.[8]

The fatigue failure modes that are associated with composites are: matrix cracking, delamination, fiber breakage and interfacial debonding. The multiple failure modes, the material being anisotropic, and non linear characteristics make it difficult to fully understand its fatigue behavior.[7] Established fatigue models are based on a S-N curve which shows how many cycles the material can survive at a corresponding stress. An example of one of these plots is shown in Fig.( 1.7) along with the fatigue limit, which is where the cyclic stress is not enough to cause damage after infinite time. In fatigue, even though the stress applied for one cycle is not damaging, after so many repeated loadings, the matrix could develop cracks 
leading to progressive damage.

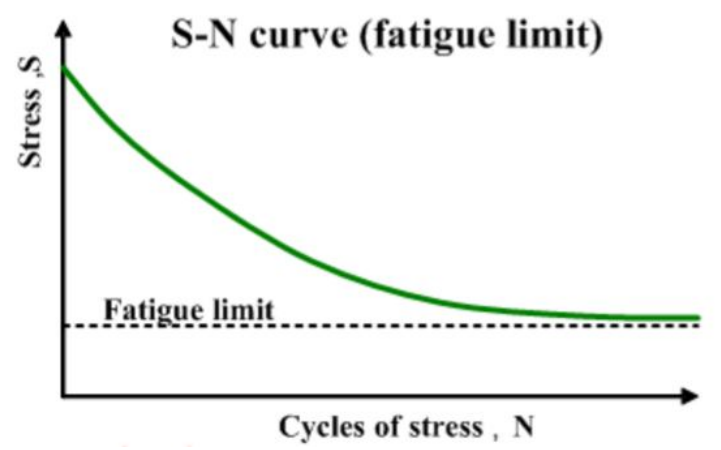

Figure 1.7: Example of a stress life curve (S-N) along with its fatigue limit [9]

Joints have also been part of the problem, and reducing the number of them could lead to greater weight and cost reductions. It is also important in design to know what kind of loads are being applied, such as tension, compression, or shear in these areas . [8] Peeling forces in these areas must also be avoided since adhesives are optimized for shear loads.

Different fatigue cycle profiles can be studied such as tension - tension, and tension - compression loadings. The most common studied is the fully reversed loading, where the structure is loaded by the same magnitude in either direction. It can be seen in Fig.( 1.8) that the stress goes up to $\sigma_{\max }$ and then reverses back to $\sigma_{\min }$ for a certain amount of cycles.[10] Note that the cyclic load can also be driven by displacement, which in that case, the equivalent to stress would be strain. It also needs to be decided at what test frequency these loading cycles would be applied to the structure. The loading, which could be a force or displacement driven, may also be scaled by a certain value. 


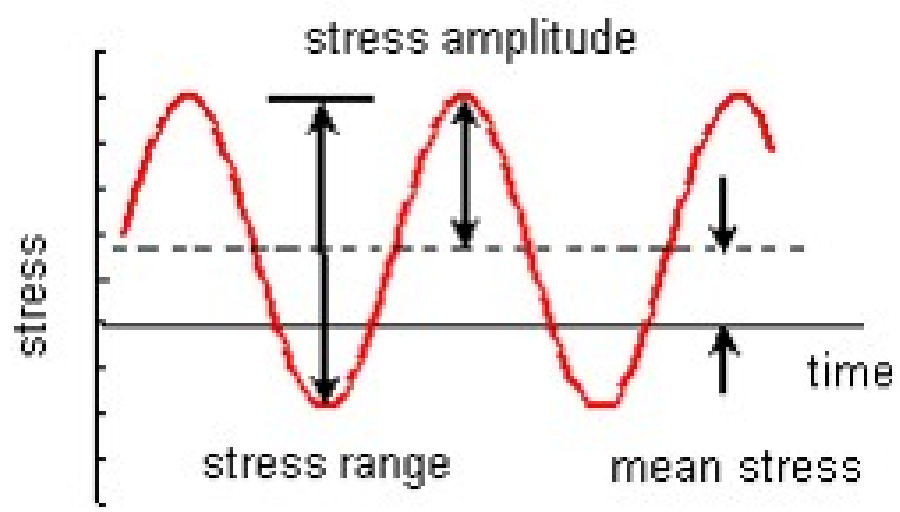

Figure 1.8: Example of a stress driven fatigue cycle and its amplitude definitions

\subsection{Previous Research on Fatigue Behavior of Composites}

In the study by Movaghghar at the Kharkov State Polytechnic University in Ukraine theoretical and experimental results for fatigue strength of a composite were compared. An energy based model for predicting fatigue life was developed using analytical equations. The equation developed for the number of cycles to failure was a function of the stress amplitude, modulus, and constants to define damage progression. The modulus was found by non destructive testing by a resonance frequency method using a shaker as picture in Fig.( 1.9). Bubble one in the figure shows the specimen that was shaken and bubble two shows where the shaker applied the cyclic displacement. After the theoretical results were calculated based on the material properties the test samples were made. 


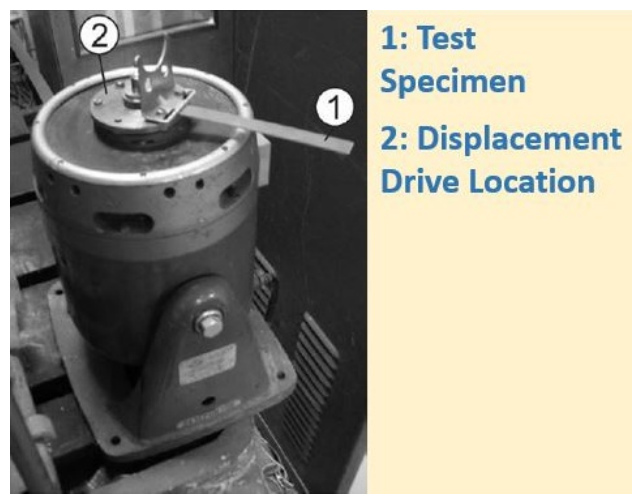

Figure 1.9: Shaker used to apply a resonance on a test sample so that the modulus could be calculated [7]

The samples that were studied were made of 10 layers of glass fibers impregnated with a phenolic resin. The cross-sectional dimensions were $2 \mathrm{~mm}$ by $15 \mathrm{~mm}$ and with a length of $175 \mathrm{~mm}$. Two groups of specimens were studied; one being cut in the warp direction of the material and the other in the weft. The two directions described can be seen in Fig.( 1.10) for clarification.

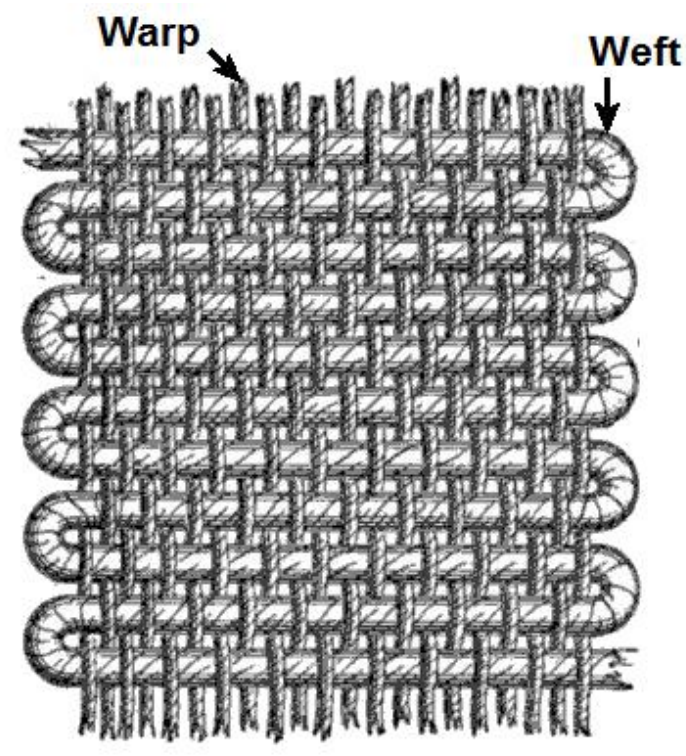

Figure 1.10: Visual of the warp and weft directions in a fabric [11]

The testing apparatus that was used is a DP-5/3 bending fatigue machine. It holds one side of the specimen fixed at location A in Fig.( 1.11b), while the moving 
clamp applies oscillating bending angles at location B. The bending angles that were tested for each group were $60,50,45,40,35$ and $30^{\circ}$. Five specimens were tested at each angle, at a test frequency of 100 fully reversed cycles per minute until failure. The results from the experimental testing were then compared to the theoretical results that were calculated.

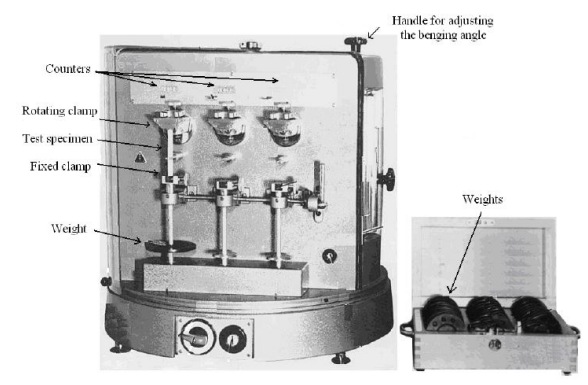

(a) DP-5/3 machine used to apply bending fatigue on test specimen

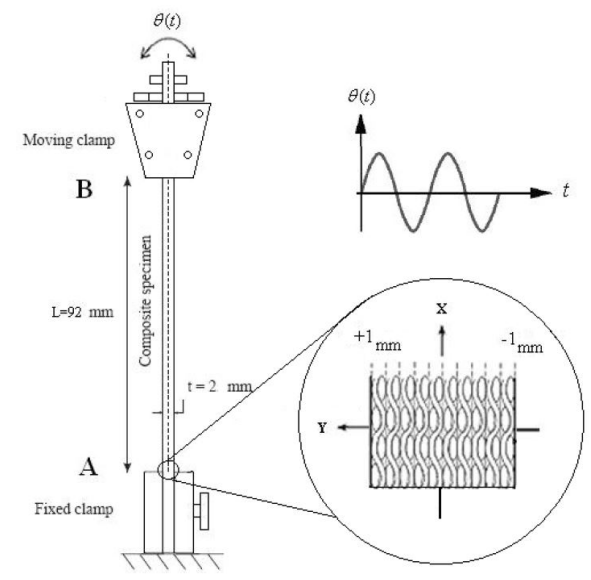

(b) Schematic of bending fatigue test setup

Figure 1.11: DP-5/3 bending fatigue machine and its test diagram

The model was able to predict fatigue because it took into account the stresses in the principal directions relative to the planes of elastic symmetry. The paper concluded the model was reliable because the theoretical and experimental curves were in good agreement.[7]

A student at Cal Poly San Luis Obispo, Ahmad Amini, also conducted a study on fatigue loaded test specimens. In Ahmad Amini's paper, he studied the effects of having notches on static and fatigue testing of composite specimens under tension. The testing was done at Cal Poly San Luis Obispo at the Aerospace Composites Lab. The specimens were made out of woven carbon fiber manufactured by Advanced Composites Group. Notches were cut into some of the specimens using a saw blade and tested statically and under fatigue.[12]

It was found in Ahmad's paper that double edge notched specimens followed a 
Griffith's model, which relates notch size to the fracture stress. This was strange to the author because the carbon fiber specimens were not homogeneous which the model is intended for. The S-N curve for the fatigued un-notched samples did follow a logarithmic relationship, which is expected. The notched samples showed a lot of scatter in the results for the fatigue life. The author concluded that it was difficult to predict the fatigue life of the specimens, since some of them would last a million cycles, while others survived only a couple thousand cycles.[12]

\subsection{The Main Objective of this Study}

The goal of this paper was to study how changing the internal structure of the wing would affect bending resistance for static and fatigue testing. The design of the wing was based on models found in the Aerospace Composites Lab as well as historical references for aircraft. The wing model consisted of a wing skin, two spars, and ribs at each end. Three wing models of the same design would be made to check consistency of the testing. Strain gages placed on the wing skin were used to check the validity of the ANSYS FEA model compared to the experimental testing. Once the FEA model was validated, it was used to simulate the testing of additional spar deigns created in SolidWorks software. The optimal spar design for static and fatigue bending loads was then determined.

\subsection{Scope of the Study}

In this study, the wing and fixture design, manufacturing, testing, and experimental and numerical results are covered.

The second chapter goes over the process of designing the wing model and the associated fixtures and mounts for the fatigue testing. Also described are preliminary fixture designs that were not produced because of issues and 3D printed prototype parts that were made. 
In chapter 3 , the process of making the molds and wing model parts is covered, along with how each part was bonded for final assembly. Following this, the method that was used to apply the strain gages onto the wing models is described. Strain gages were bonded in three distinct areas on the wing skin including on top of the leading edge spar, trailing edge spar, and along the chord, 2.5 inches away from the root.

The fourth chapter covers details of the DAQ hardware and software, since strain gages are used throughout the testing process. It also covers the testing conducted including material properties, FEA validation, static testing of the wing model, and of the fatigue testing. It also explains the analytical solution for the composite cantilever beam, so that the results could be compared to the experimental and numerical solutions.

Chapter 5 goes over the results of various static test that were conducted on the wing models as well as the composite cantilever beams. The cantilever beam testing was done in order to be assured that the material properties, boundary conditions, and loads are modeled well in the FEA model. Then, the results of various dynamic test are covered including the fatigue life test until 100,000 cycles or failure.

In Chapter 6, the thought process, model creation, and results regarding the FEA models are covered. Specifically the FEA models for the composite cantilever beams, static and fatigue testing for the wing model were explained.

In the last chapter, the experimental and numerical results are compared for the validation studies, and static testing. In this chapter, the conclusion and future work are covered as well. 
Chapter 2

DESIGN METHODOLOGY OF WING AND FIXTURE

Designing the wing parts and fixtures used for testing involved multiple processes. Design ideas were taken from historical aircraft, models in the Cal Poly Composite Lab, and advisor recommendations. This chapter covers the design process for the wing design, presents the final wing dimensions and CAD models, and closes explaining the wing fixture design process.

\subsection{Wing Design Thought Process}

A symmetrical airfoil would be used in order to simplify the manufacturing process. This allows the upper and lower wing surfaces to be fabricated using the same mold. A NACA 0016 airfoil was selected for the reason that it provides a maximum thickness under 1.5 inches. Limiting the maximum thickness of the wing profile limits the wing mold size, and in turn limits the material and machining costs of the fixture.

Overall length of the wing was limited due to space limitations in the composites lab. Since the wing was cantilevered off a fixture, and attached to the Instron, excessive length is not ideal.

Chord length was largely influenced from existing models in the composites lab. The metal wing pictured below Fig. ( 2.1) was used as an example for the chord length of the composite wing. The metal wing is regularly used for experimental testing at Cal Poly, in an aerospace department lab course. 


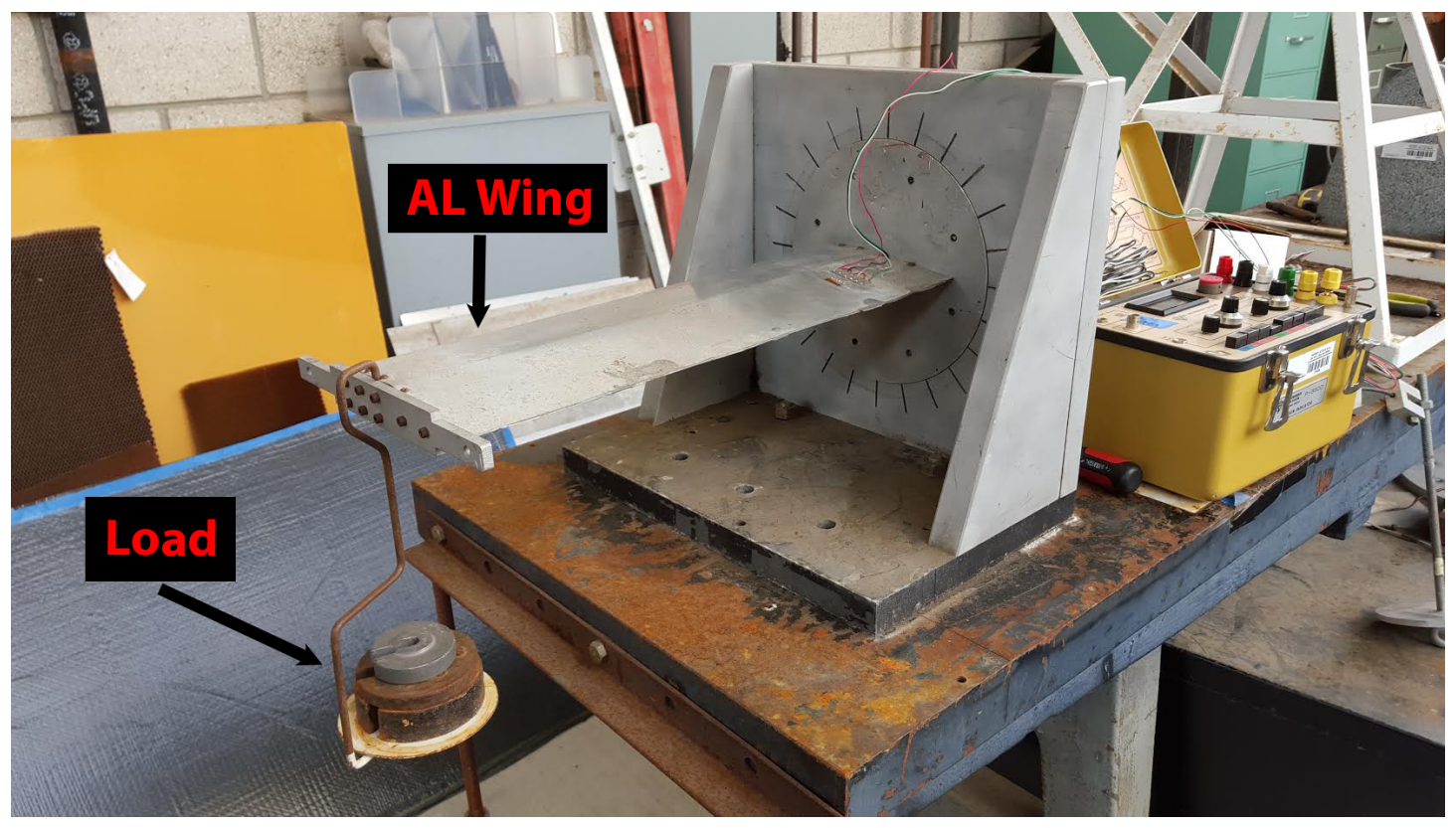

Figure 2.1: Metal wing used in experimental analysis course at Cal Poly - San Luis Obispo

The leading and trailing edge spar locations were chosen, based on historical reference passenger transport aircraft. Since the spars fit inside the skin, their length and height were driven by its dimensions.

Many different types of spars can be used in aircraft, and each has its own pros and cons. The cross-sectional shape of the spar was chosen based on what is used on aircraft. Considerations for the spar design was also influenced by the manufacturing process. In order to make the spars, a mold would have to be made that would allow for the part to be removed after curing. Initially an I beam design was going to be used, but complications arose from needing the flanges to match the angle of the wing skin.

It can be seen in Fig.( 2.2) below that one side of each spar would close at an inward angle on the mold, while the other side would flare outward. The inward angle would make it extremely difficult to remove the mold from the cured part, even if mold release was used. The I beam cross-section spars are shown in black, while the molds with the issue are shown in blue. Since the purpose of the physical 
model was to solely validate the FEA model, it was decided to make the spars a $\mathrm{C}$ - channel design. This type of spar is also used by many aircraft manufactures, and would make the demolding process far easier.[13]

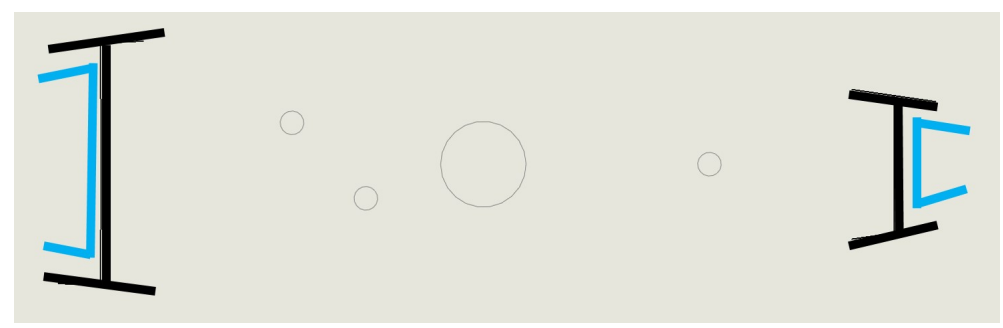

Figure 2.2: I beam spars enclosing on part mold

It was not beneficial for the wing to be either too stiff, or too flexible. Making the parts too thick, would have made the strains and displacements a small magnitude, making it difficult for data collection. On the contrary making the wing flexible, would have raised the likely hood of the wing not breaking due to the fatigue loads. Flexibility would have allowed the wing to freely deflect with the Instron actuator, and not be stressed as much as a stiffer design.

The number of layers of carbon fiber that were used in the various wing parts was determined for different reasons. The average cured thickness of this material was found to be around 0.008 inches. While the cured thickness of the Hexcel material was thicker at around 0.01 inches.

The structural purpose of the wing skin, is to not allow the internal structure to buckle or twist during various types of flight loads. The wing skin also needs to be a smooth surface for aerodynamics, but was not of concern in this thesis. It was decided that the wing skin would be two layers, allowing it to provide support without being excessively stiff.

The spars purpose is to provide bending stiffness of the wing, in order withstand the wing aerodynamic forces. Most of these forces are in the vertical directions making the wing bend upwards or downwards. Therefore, this is the most promi- 
nent load path to the fixed constraint at the metal fixture. Different types of shapes can be used for spars, as shown in Fig.( 2.3). The leading edge spar was made using three composite plies, since this is where the wing would be loaded. The secondary load path would be the trailing edge spar, which was made with 2 layers of carbon fiber. Not as much load would travel through the trailing edge spar unless there is considerable twist in the model. Both of the spars were made of the Cycom carbon fiber.[4]

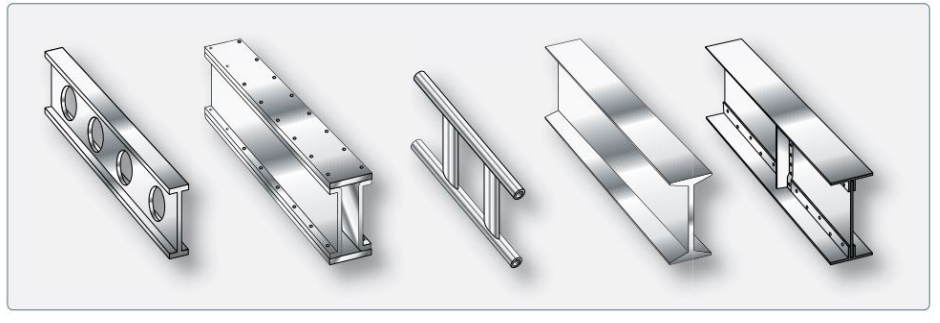

Figure 2.3: Different types of spars used in aircraft

Lastly, the end cap ribs were made extra thick to provide support at the constraints and mounting areas. Reaction bending moments and forces would be centered at the root rib therefore it was important to reinforce this area. Screw holes and T-nuts were also bonded on this part which would lower its nominal strength. For this part 10 layers of Hexcel material was used to make the flat plates from which the ribs were cut out of. Thicknesses did differ between layups, but it was not significant enough to introduce considerable error in calculations, and FEA analysis. After testing, it was found that the wing part dimensions yielded a moderately stiff wing but lightweight.

\subsection{Wing Design}

The final wing design dimensions, based on considerations, are outlined in this section. The NACA airfoil that was chosen, was a 0016 symmetrical airfoil, with a maximum thickness of 1.48 inches. The wing length was chosen to be 15.25 " 
from root to tip. The maximum thickness of the wing was calculated from the chord length of the airfoil which was 9.25 inches. The leading and trailing edge spar locations were $22 \%$ and $72 \%$ respectively. These percentages equated to a distance of 2 inches and 6.7 inches from the nose of the rib respectively. An exploded view of the wing can be seen in Fig.( 2.4).

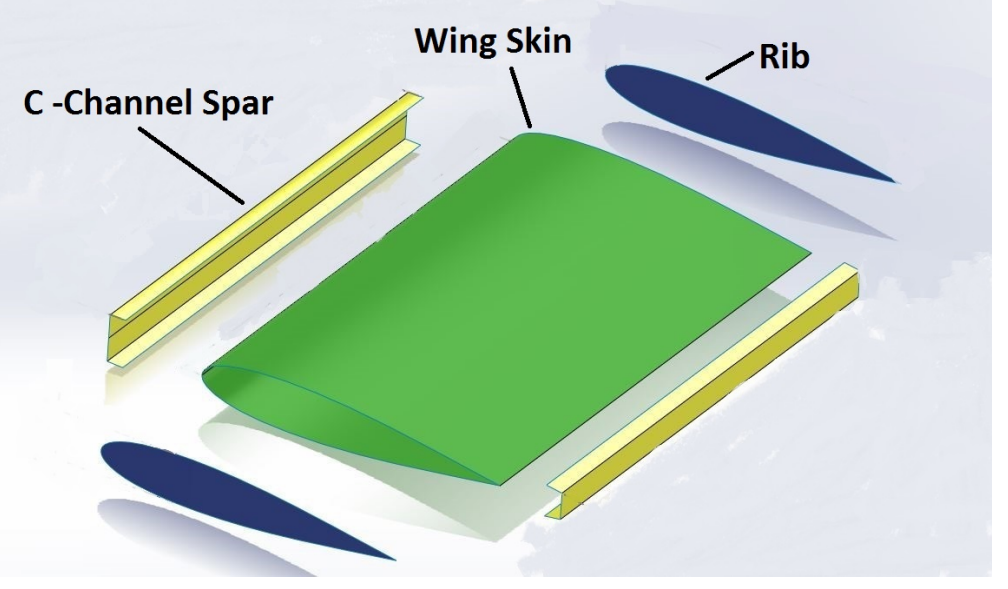

Figure 2.4: Exploded view of the wing

A C- channel spar design was chosen to ease demolding of the cured part. The open side of the C - channel was faced inward for each spar. A SolidWorks representation of the spars orientation, and placement is shown in Fig.( 2.5). The location of the spars can also be seen with reference to the leading edge of the wing.

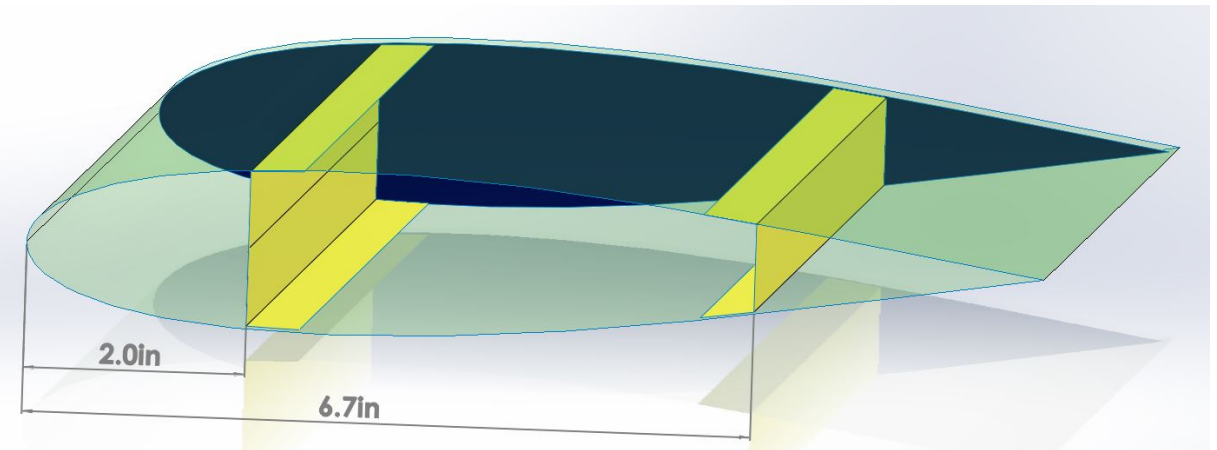

Figure 2.5: Spar design

The dimensional thicknesses of the wing skin, leading edge spar, trailing edge 
spar, and end ribs were $0.016,0.024,0.016,0.1$ inches respectively. Height of the spars and flange widths can be seen in Fig.( 2.6). The angle of the trailing edge spar flange, with respect to the horizontal plane is also shown. The flange was angled so that it would follow the contour of the wing skin. Since the leading edge spar was placed near the maximum thickness of the wing, there was a small angle at this location.

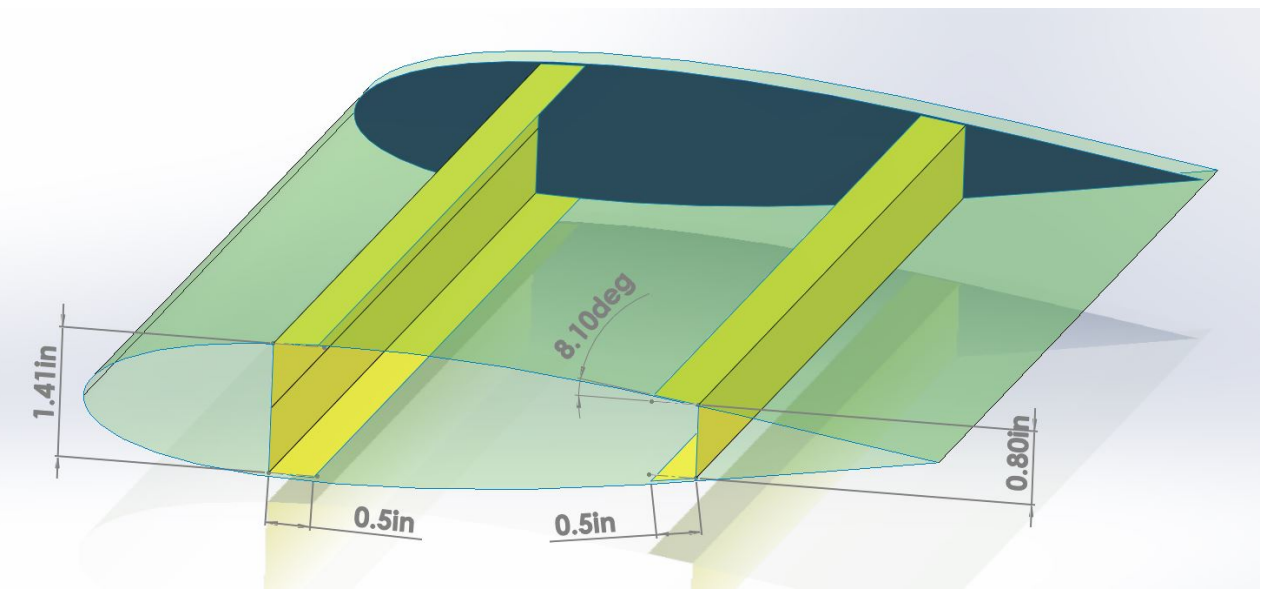

Figure 2.6: Spar dimensions

A summary of the wing details can be seen in Fig.( 2.7). It also shows a representation of the wing model, with a section cut of the skin, so that the internal structure would be visible. All fixtures would then be designed for this geometry and shape.
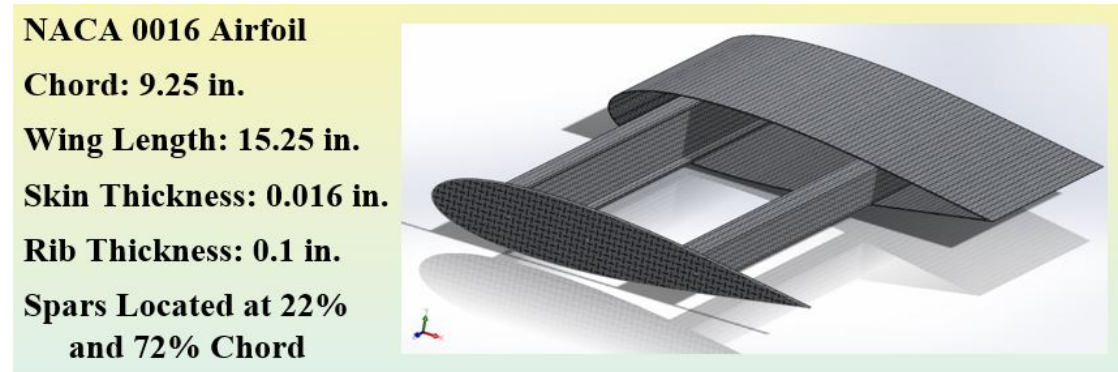

Figure 2.7: Summary of wing design details 


\subsection{Wing Fixture Design Process}

Many iterations of the wing fixtures were designed, and even made, before the final iteration. The first subsection shows what thoughts went into the design

process of the fixture, for this thesis project. Following this the initial design of test fixtures is covered, even though they were not used for testing. A 3D printer was also utilized in order to fast prototype fixture parts. This practice has been put into use by engineering companies in recent times. Then the final fixture used for data collection is explained.[14]

\subsubsection{Fixture Design Considerations}

To test the wing as a cantilever structure under static and fatigue loads a fixture would need to be made. The fixture will consist of a rigid base, and a mount for the root and tip ribs. Since small displacements and forces would be put on the wing, rigidity in the fixture was highly important. If the fixture was not rigid, the displacement of it would have introduced considerable errors into the data. Therefore the fixture must be able to take much more load than what would be applied to the wing. The fixture also needs to have an attachment point for the wing, such as a flat plate with screw holes or some type of clamping area. This attachment would need to have an interface with the rest of the fixture. The fixture would also need some type of machine work that would need to be done, which would add time to its manufacturing process. Although, since metal is the preferred material for rigid fixtures, machining is inevitable.

Since the wing tip needed to be attached to the Instron grips, a mount would need to be made to accomplish this. The mount would needed a way of being attached to the end of the wing model, without adding considerable load onto it. This mount needed a flat area so that the grips on the machine could hold the mount, and apply a force or displacement. This flat area was made out of a 
material that would not be crushed by the hydraulic grips. The mount needed a hinge or pivot point that would allow the wing to move laterally, when displaced vertically. Since the actuator on the Instron could only move in a vertical manner, it would apply an axial force to the wing, if it is not allowed to pivot. This would introduce additional stress, besides the bending stress which is of interest in this paper. Due to vibration from the fatigue testing, any bolts or screws would need locking washers, so that they do not loosen. This was an easy solution to a problem but important nonetheless.

\subsubsection{Initial Designs}

The initial fixture design was intended to be bolted to the Instron base, and hang off of it with the use of steel beams. This design can be seen in Fig.( 2.8), where a frame structure would be bolted or welded at the joints. The wing would be simply bolted to the aluminum plate between the vertical supports. The tip of the wing would be attached to the Instron, with use of a door hinge so that the it would be allowed to displace horizontally.

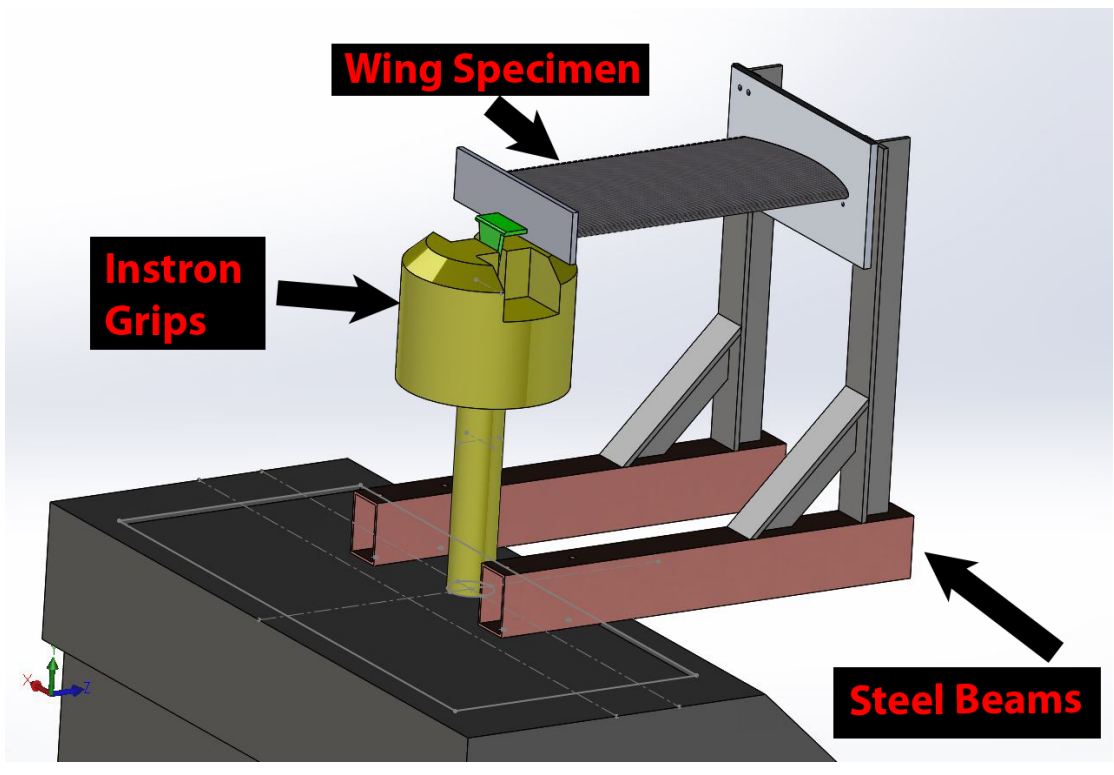

Figure 2.8: Initial hanging fixture design 
This design was ultimately not made, because the steel beams to be used, were excessively heavy. The concern is that the steel beams would displace from its own weight. The beam member lengths were considerable, that they would deflect due to the weight of the beams, as well as the testing loads. Different iterations of this type of frame, were designed to solve these issues. Although, it was found that a completely different design would need to be used. Due to this problem it was decided to place the fixture on a rigid stand. This was a great solution and after research it was decided that a $50 \mathrm{lb}$ angle plate would be bought, and used to hold the wing fixture. The angle plate had a 10 in by 10 in face, where holes could be drilled to bolt onto. The angle plate that was acquired, is shown in Fig.( 2.9) below.

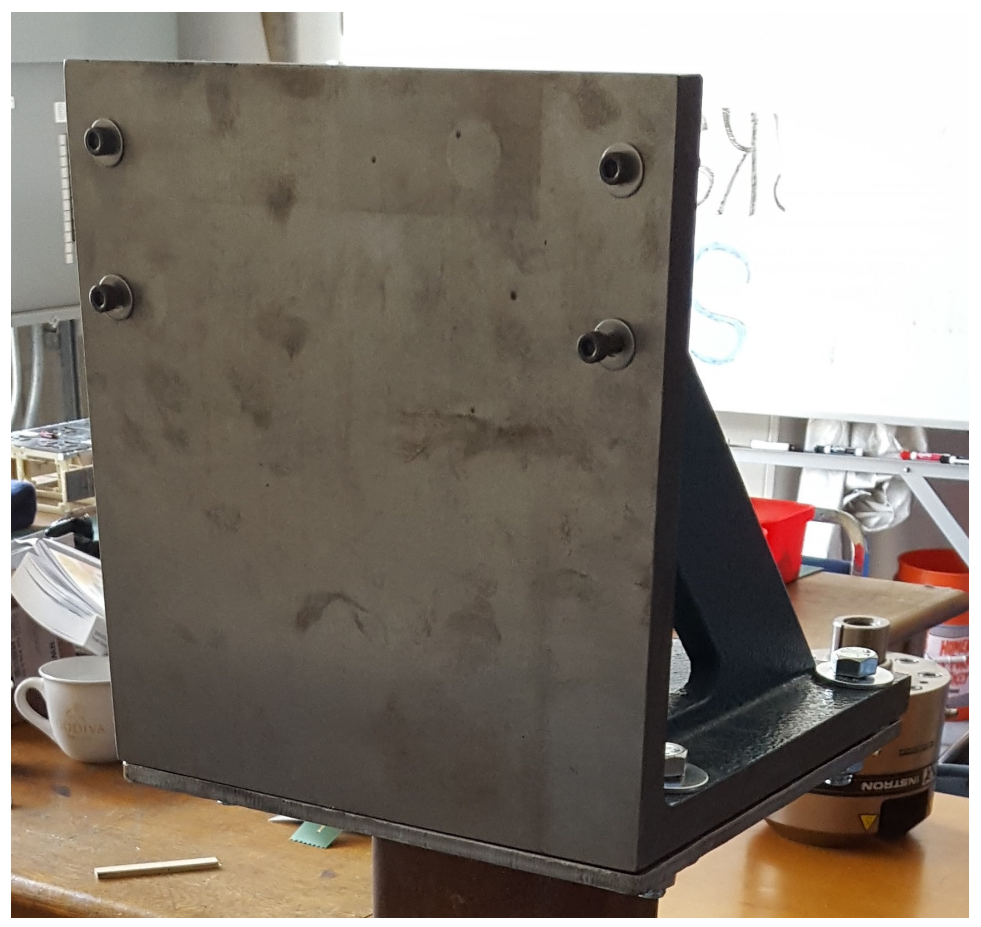

Figure 2.9: $50 \mathrm{lb}$ angle plate used to hold the final wing fixture

The interface with the wing root, was found not to be ideal, based on experimental testing. This iteration merely had 5 bolts that would screw into $\mathrm{T}$ - nuts bonded onto the inside of the wing. A test was done using the circular metal plate, 
used for the metal wing from the aerospace experimental stress analysis course at Cal Poly. The wing was screwed into the plate and a force was added until failure as shown in Fig.( 2.10).

After conducting this test, it was observed that the wing broke at the root connection after a relatively small force. It was decided that the peel condition on spar to the rib face bond area caused the failure. Adhesives are optimized for shear conditions, so it is beneficial to design bonded areas to be loaded along surfaces. The actual carbon fiber material would have withstood a much higher load, if it weren't for the bond failure. In order to solve this issue it was determined that the spars or skin, would need to be constrained instead of solely the rib face. This initial wing also did not have a wing skin helping to resist the torsional loads. It can be seen in the picture that the leading edge spar was twisted off, because of the torsion. For any future test the wing skin would be included in the assembly to prevent this issue.

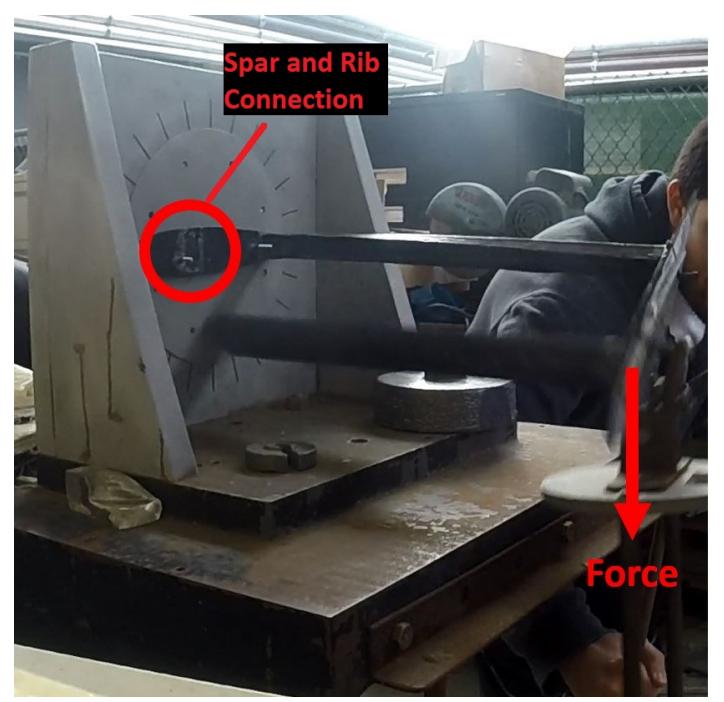

Figure 2.10: Picture of prototype wing failure at the root and spar connection

The next proposed design to constrain the wing at the fixed side, was a type of gripping fixture. Two blocks on each spar would be tightened down onto the spars with bolts. This would solve the bonding issue altogether since the root rib 
would be removed. The protrusions of the blocks would clamp onto the spar web by friction during testing, as shown in Fig.( 2.11) below. However, this design was not made because of issues with stress concentrations at the gripping area and the edges. This design also did not constrain the wing skin in any way, which would basically removed its load path all together. Therefore the final fixture design used a clamping design that would clamp down on the outside of the wing, constraining the skin, spars and rib.

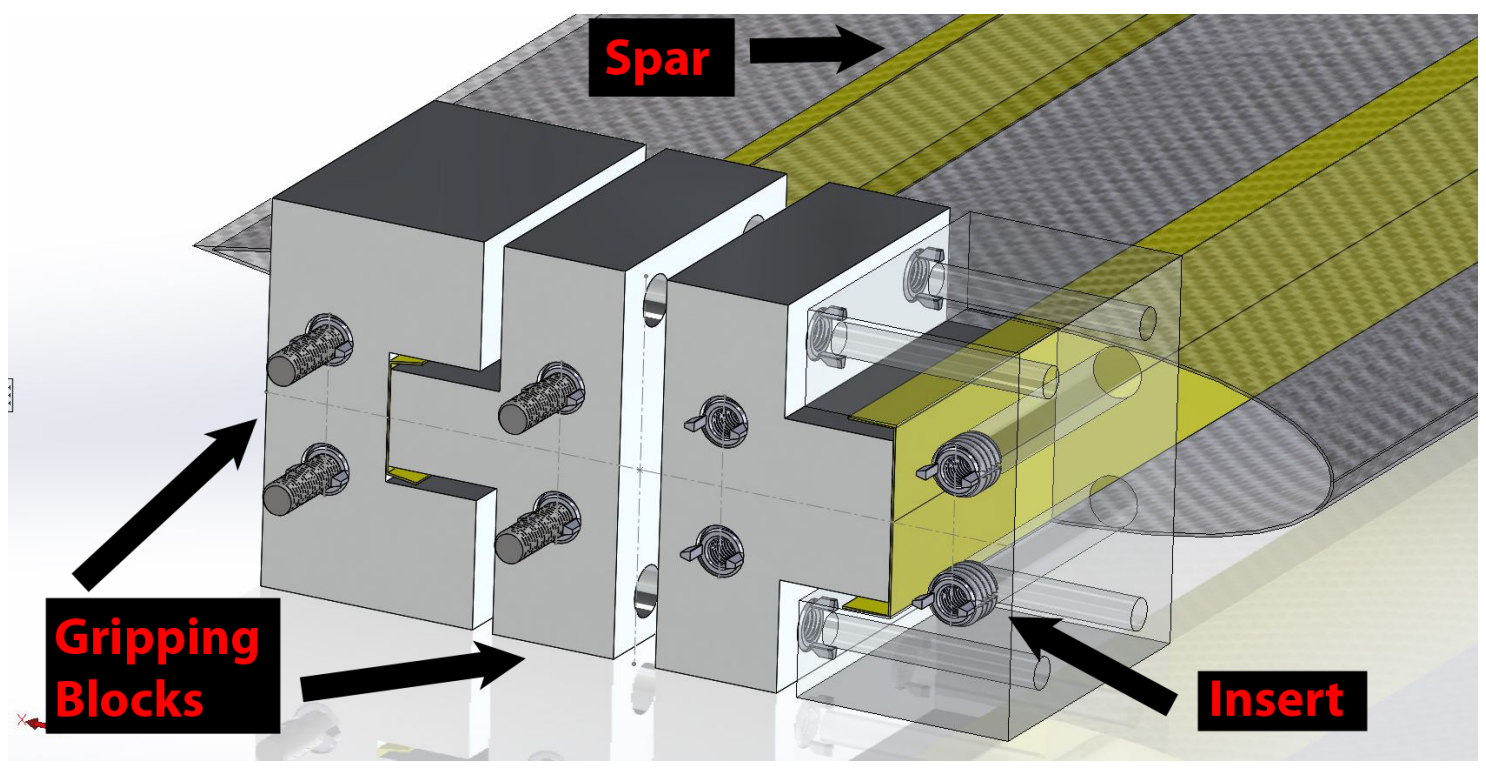

Figure 2.11: Solidworks model of the gripping fixture design

An aspect of this design was implemented in the final fixture, which was the use of locking key inserts for the screws. Since the aluminum is far easier to machine, it would be the best material for the fixtures. Although, since the screws were made out of steel which is a harder metal, it would cause damage to the aluminum threads in the fixture. This is especially true for fatigue testing, where there are vibrations induced and the load is repeated many times. The inserts allow for the bolt and the threads to be the same material so that they do not get worn out. The key inserts are threaded into the fixture material and its pins are pressed in to lock it into place. The inside diameter of the insert uses the threads of the bolt 
that is desired. A key insert before being thread in, as well as locked in can be seen in Fig. ( 2.12). Washers were also used for all bolts in the fixture to provide adequate fastener pressures.

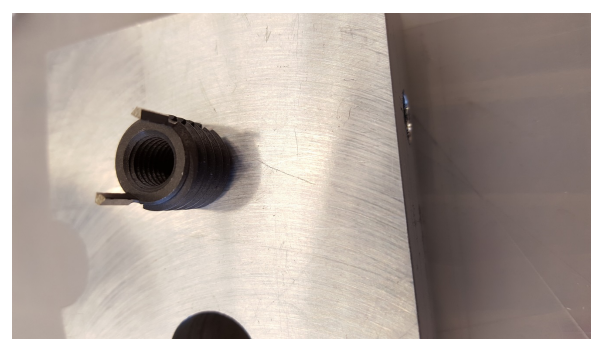

(a) Insert being threaded into part

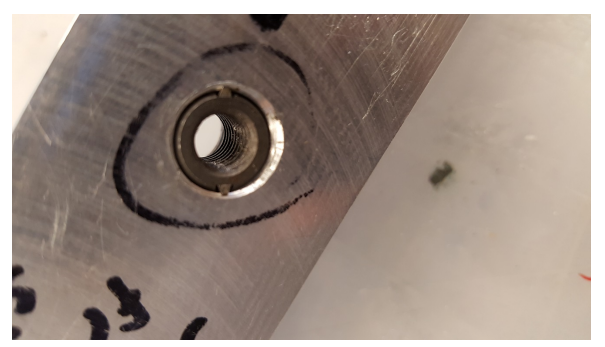

(b) Installed key locking insert

Figure 2.12: Locking key insert in final fixture part

In order to make sure that the wing mount, would fit as desired it was decided to make 3D printed prototype parts. Considerations that had to be taken into account when printing, are tolerances of dimensions for through holes and slots. Since on the first prints the tolerances were not taken into account, parts did not fit together well. Since the fit between the parts was important the solution was to begin to print parts but stop them a quarter of the way through. This allowed for the fit between parts such as a dowel and a through hole. If the fit was not ideal then the part would be reprinted with a different dimension until the fit was adequate. This was not the most time efficient process but allowed for the fit that was desired.

A part in progress and the prototype mount can be seen in Fig.( 2.13) directly below. The mount consisted of a flat plate with holes for screws, so that it could be used to attach to the wing tip. A mount for the pivot point at the free end of the wing was also made. This pivot piece was attached to the plate by $\mathrm{T}$ - nuts which fit into the horizontal slots. Lastly a flat paddle piece was made, where the square are was intended for the Instron grips to clamp onto. At the opposite end there was a hole so that it could be held onto the mount with a pin, allowing the 
wing to pivot. The final mount fixture was very similar to the prototype that was made by $3 \mathrm{D}$ printing. The only change for the final mount was the material for increased rigidity, and dimensions because of the higher strength.

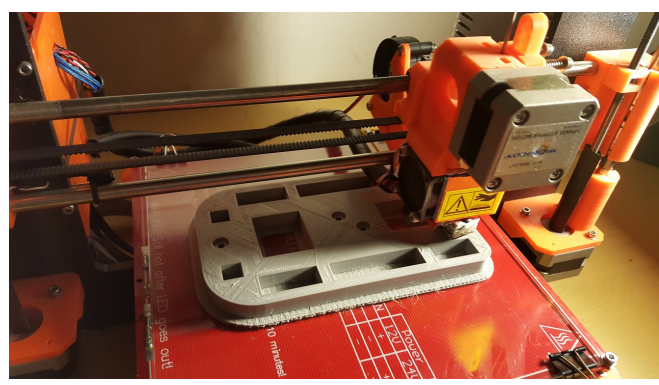

(a) Prusa 3D printer

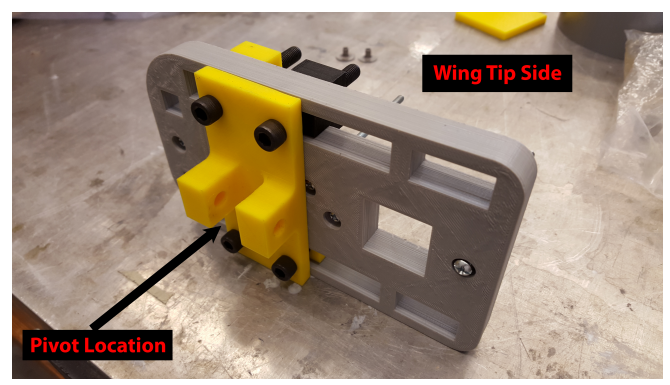

(b) Prototype of front mount

Figure 2.13: Prototyping front mount

\subsubsection{Final Fixture Design}

As mentioned in the previous section, an angle plate was ultimately used for mounting of the root rib mount. Four holes were drilled into it so that bolts would be used to attach the fixture. The next issue was what the $50 \mathrm{lb}$ angle plate would rest on. The solution was to weld two steel plates onto a steel square column for the base. The base was made to a height where the wing tip mount would fit into the Instron grips half way through its travel. Therefore, the Instron would be able to be displaced to its maximum in either vertical direction. Then holes were drilled holes in the floor, so that inserts could be used for the bolts that were used to secure the base. There were also holes on the top plate, so that the angle plate could be secured, as shown in Fig.( 2.14). 


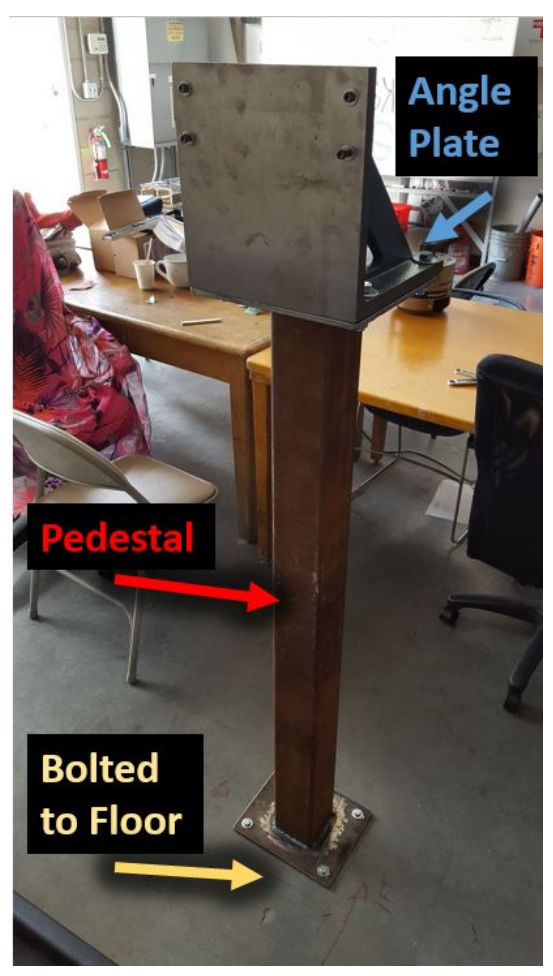

Figure 2.14: Mounting fixture for the wing

The final design for the root rib mount, ended up being a thick aluminum flat plate, that would be bolted to the angle plate. Four bolts and nuts were used to attach the plate to the $50 \mathrm{lb}$ angle plate. Washers were used throughout the fixture to distribute the bolt pressure efficiently. This plate would also have 5 holes, so that screws could be threaded into $\mathrm{T}$ - nuts inside the wing. This would constrain the rib against the plate.

Constraining the face of the wing to the plate, would not solve the problem of the peel condition on the adhesive. Therefore it was important to figure out how to change or remove the stress at the bond. The best way to do this was to make two halves of the airfoil shape, which would be used to clamp down onto the wing with bolts. Since the clamping parts were 0.725 in thick, they would move the highest stress away from the bond area to the edge of the clamp. Since at the edge of the clamp there was only carbon fiber material, it would be able to take the reaction forces and stress at the constraint. An exploded view of the fixture 
parts, along with dimensions can be seen in Fig.( 2.15).

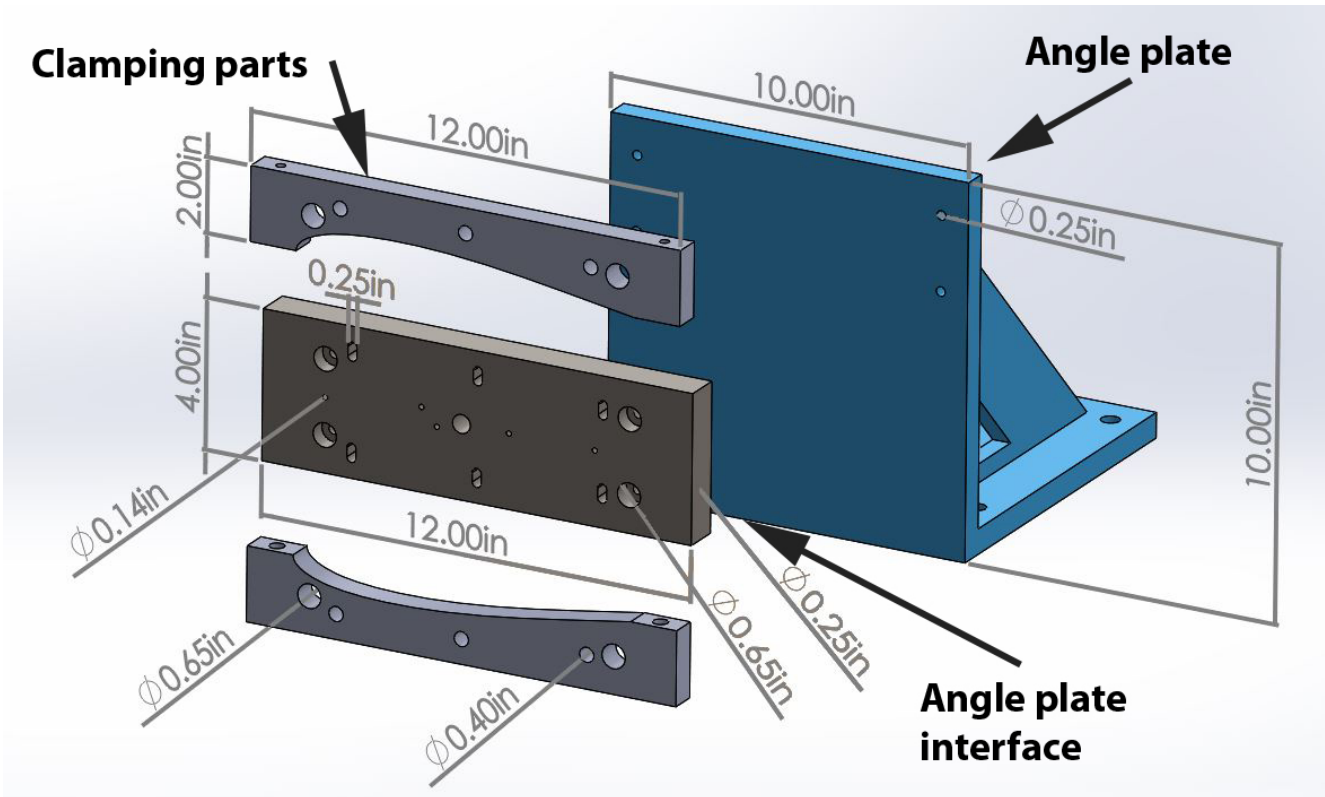

Figure 2.15: Fixture part dimensions

This aspect also allowed the skin to be fixed by friction on the clamping parts. The clamping parts, as well as the metal rib explained in the following paragraph, are shown in Fig.( 2.16a). In order to support the clamping force, an aluminum rib was placed at the root side. Fig. (2.16b) shows how the metal rib would rest up against the plate, and the carbon fiber rib would be offset from it. The wing model would have the carbon fiber rib bonded at a distance from the skin edge, so that the metal rib would fit.

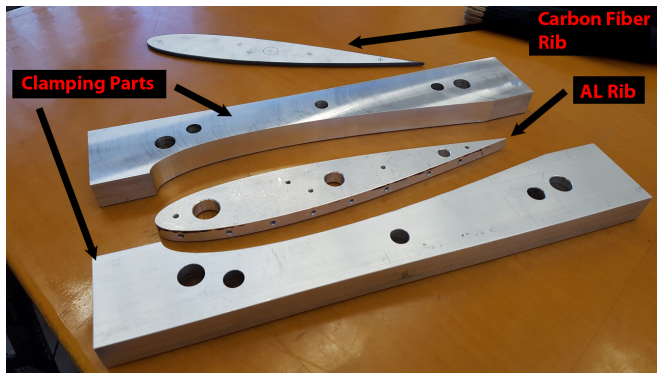

(a) Clamping parts and aluminum rib

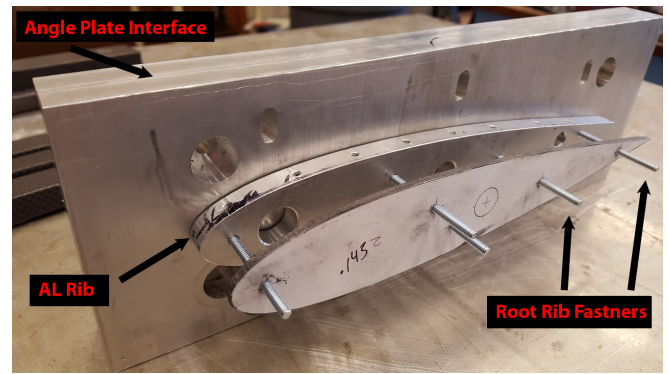

(b) Mounting plate and aluminum rib

Figure 2.16: Root side of wing model mount 
In Fig.( 2.17) an exploded view of the fixture can be seen showing how the wing interfaces with the clamping parts. It also shows all the bolts, nuts, and screw inserts that were used in the assembly. In Fig.( 2.18) the machined fixture parts are shown, spaced out with some of the interface bolts. From left to right the plate, clamping halves, aluminum rib and the root side of the wing model. Note that all of the holes on the clamping parts had locking key insert put into them. Nuts were only used for the bolts connecting this part of the fixture to the angle plate.

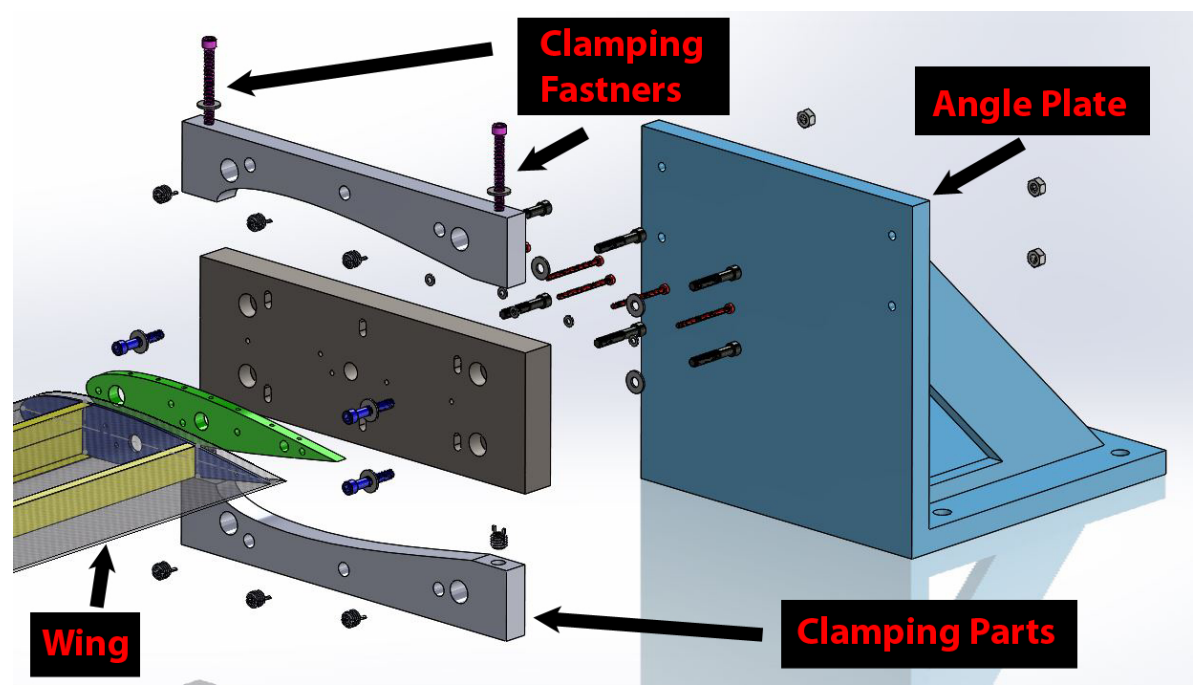

Figure 2.17: Exploded view of fixture 


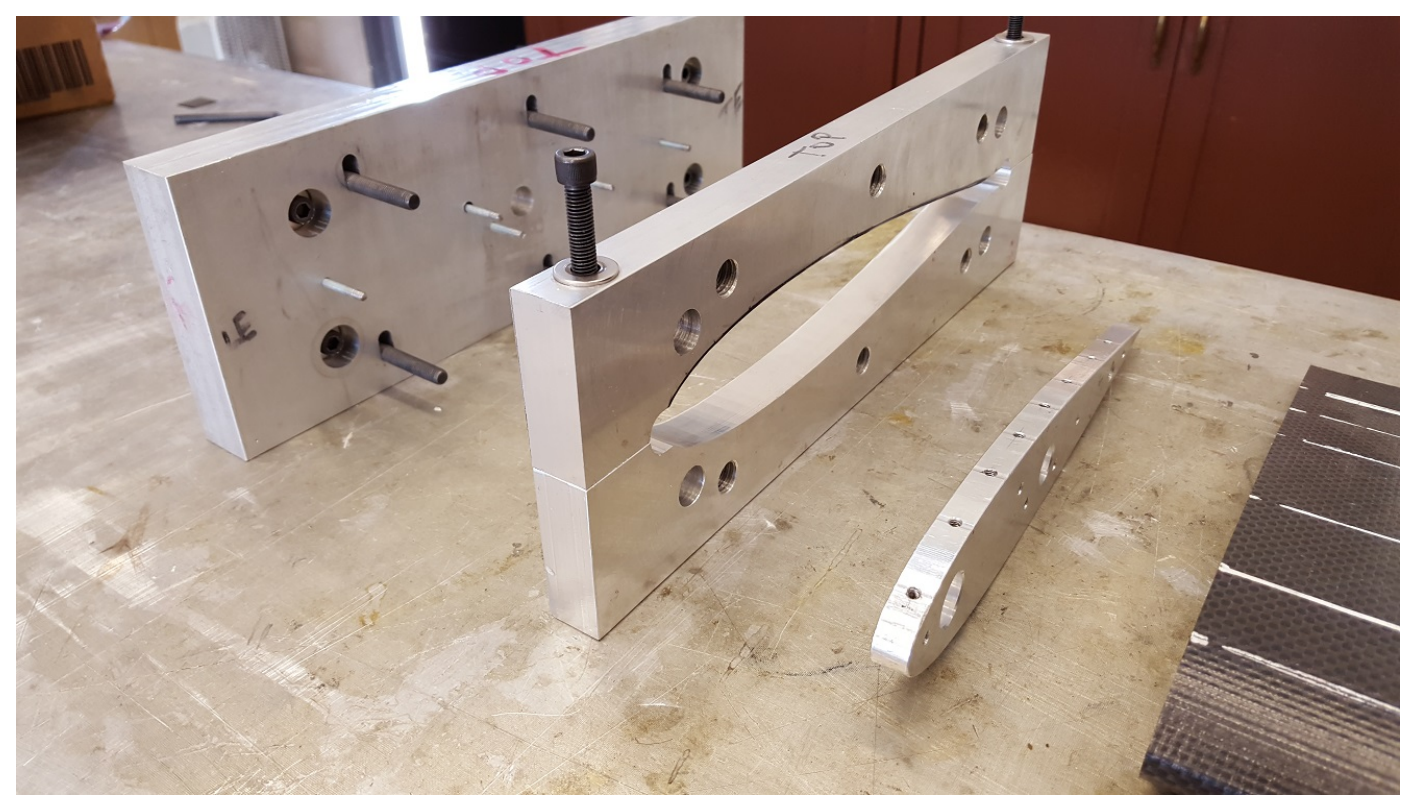

Figure 2.18: Fixture assembly

For the wing tip mount it was decided to use a thin aluminum plate with 5 holes similar to the root side connection. The final design that was made can be seen in Fig.( 2.19) along with the bolts holding the assembly to the wing. For reference the dimensions that the front mount parts were machined to, can be seen in Fig.( 2.20). In Fig.( 2.21a) the screw holes can be seen, as well as the the slots that were used to attach the pivot mount with $\mathrm{T}$ - slot nuts. The 3 square cuts were put into the plate to reduce the weight of the mount, so that it would limit the effect on the displacement results. The next part made for the fixture was the pivot mount, which consisted of two prongs with a hole through each side. A paddle shaped part was also made with a flat surface for the Instron to grip, and a thin part on the opposite end with a hole. The hole was placed between the two prongs, and a dowel was placed through it to make a hinge connection. The dowel was held in place by a nut, and the paddle was kept centered with safety wire. Even though not pictured here, lock washers were added to the screws in Fig.( 2.21b), so that they would not loosen from the vibration. 


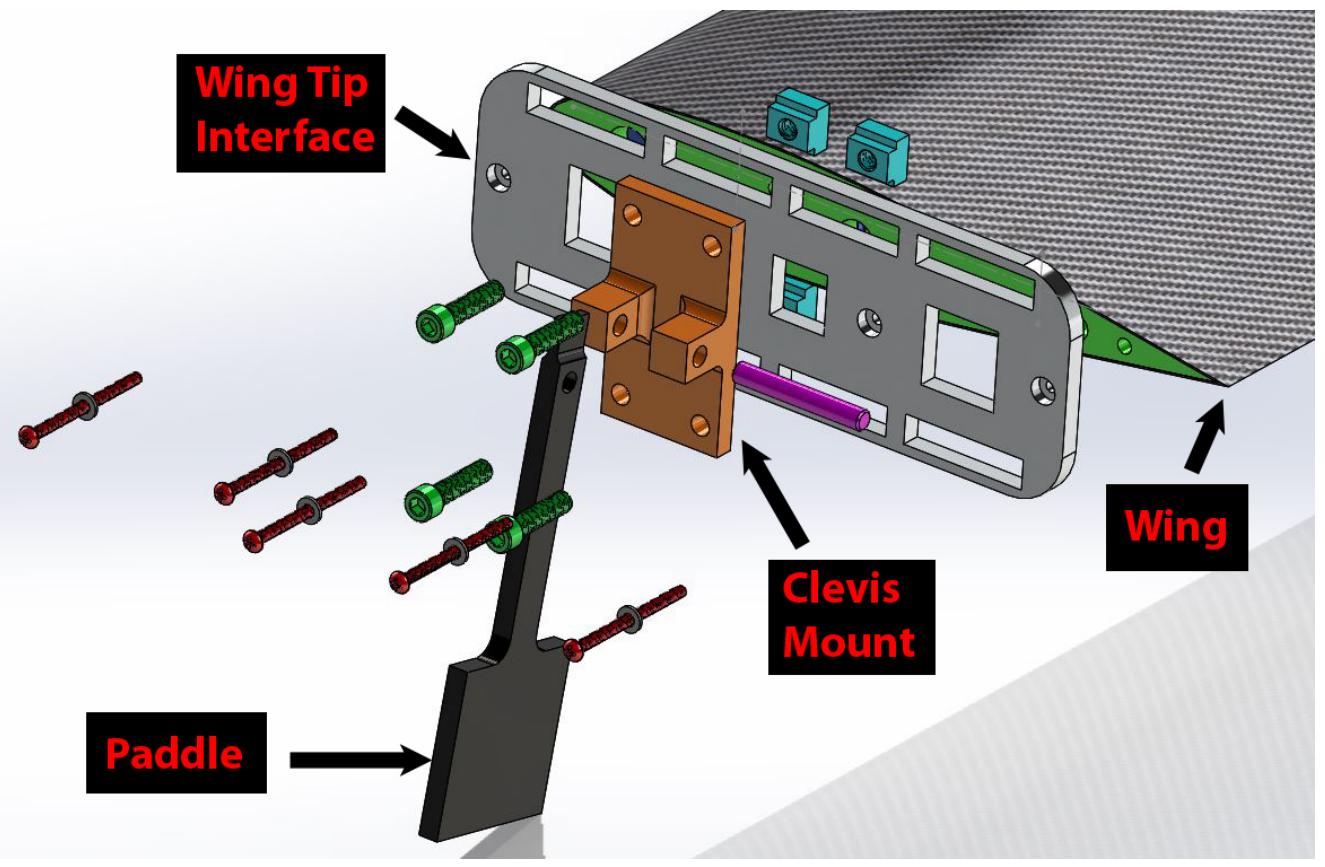

Figure 2.19: Wing tip mount assembly

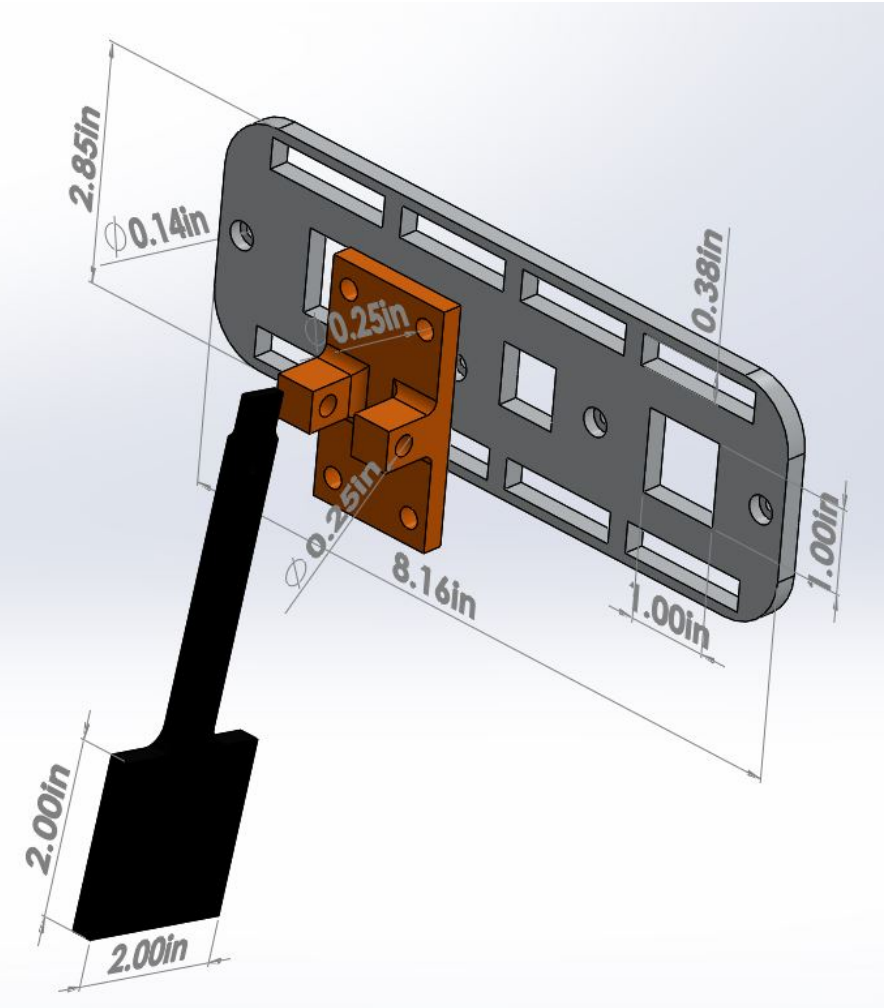

Figure 2.20: Front mount dimensions 


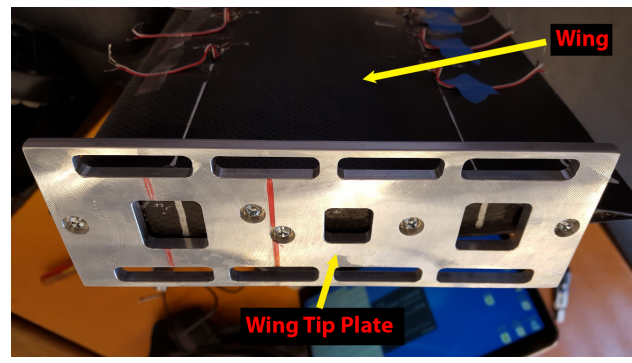

(a) Tip mounting plate

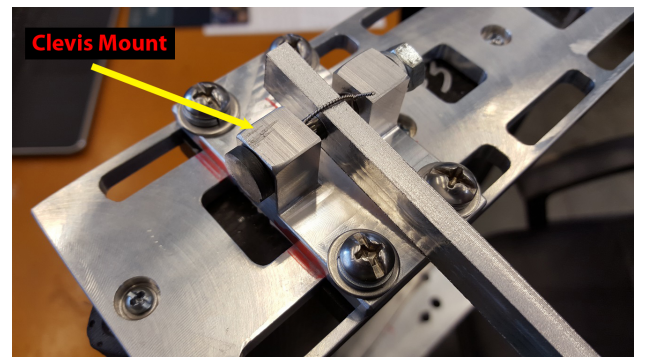

(b) Wing tip plate and pivot interface

Figure 2.21: Wing tip mount

The final design of the fixture worked exceptionally well, and closely resembled the designs made in the CAD software, shown in Fig.( 2.22). The parts ended up working, and fitting as they were intended to, when going through the design phase in SolidWorks. The final fixture assembly with a wing model can be seen in Fig.( 2.23).

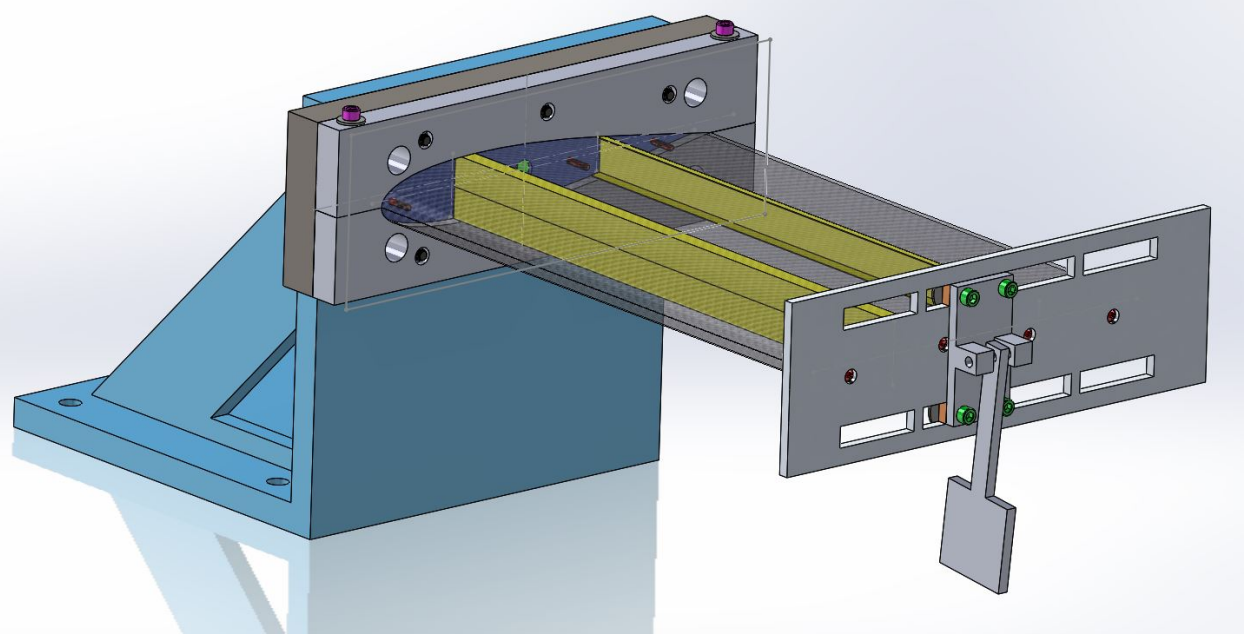

Figure 2.22: Final fatigue fixture assembly CAD model 


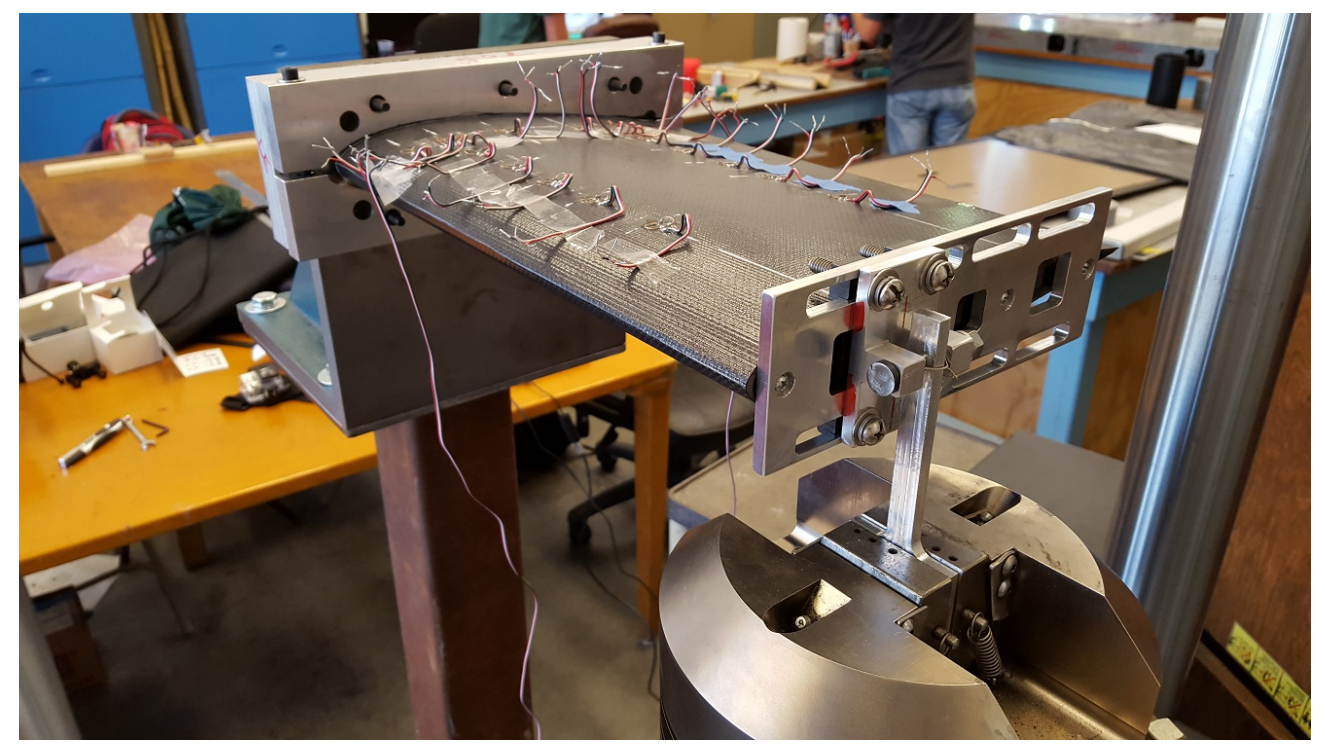

Figure 2.23: Final fatigue fixture assembly 
Chapter 3

WING MANUFACTURING

In this chapter, the manufacturing process of all the molds and parts used to make the wing model are covered. First, the curing cycles for both of the composite materials used is shown. Two different wing skin molds are explained along with the spar molds. Then, how the skin, spars, and ribs are manufactured is covered in detail. Then, how the parts are assembled and bonded to become the wing model is explained. Lastly, how the strain gages are bonded onto the wing skin is shown. 3.1 Curing Cycle for Cycom and Hexcel Carbon Fiber

For the wing parts, pre-impregnated carbon fiber weave was used from manufactures Cycom and Hexcel. In order to insure that the material is cured properly, the manufacture supplies the recommended cure cycle on its data sheet. On this sheet, the temperature and pressure that is recommended to be applied onto to the material for a full cure is shown. It also shows the duration that it needs to be under each condition. The temperature activates the resin in the raw material while the pressure removes excess resin. A heated press was used to cure flat geometry parts, while complex parts were cured in the American Autoclave Co. autoclave in the Cal Poly Aerospace composites lab. For the complex parts, a vacuum bag layup was used as well as vacuum ports to apply a vacuum.

Parts with a flat geometry, included the wing ribs and material property specimens which were made with Hexcel AGP 280-5H fabric. The carbon fiber plies were placed between two steel plates with non-porous film to provide release. Then, 
this stack up was placed in the heated press for curing. This material had a two stage cure process, where the material would be held at a certain temperature for a period of time and then increased to a higher temperature. The temperature was increased to $225 \mathrm{~F}$ at a rate of 3 degree $\mathrm{F} / \mathrm{min}$ then held constant for a dwell time of 60 minutes. After this, it was increased to $350 \mathrm{~F}$ at the same rate and held there for 120 minutes. It was then cooled to $140 \mathrm{~F}$ at the same rate as the heating, where the pressure was then released. Throughout the entire cure process, a force of $300 \mathrm{lb}$ was maintained on the flat plate. A plot of the cure cycle for the Hexcel material is shown in Fig. ( 3.1) below.

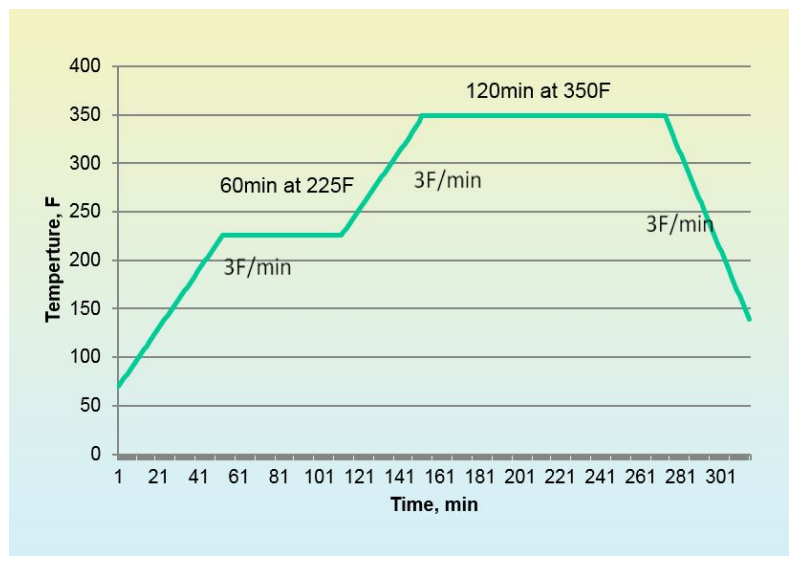

Figure 3.1: Hexcel cure cycle

In order to cure complex geometries, an autoclave would be needed since it can apply heat throughout. The limiting factor of the autoclave is that it has a maximum temperature of 250 degrees F. For this reason, complex geometries needed to be made out of the Cycom carbon fiber, which had a lower cure temp than the Hexcel. The temperature limit could be increased if nitrogen would be used, but this a costly upgrade.

The complex parts included the wing skin, leading edge and trailing edge spars. Once vacuum bagged, the layups were placed in the autoclave and connected to its vacuum hoses. Throughout the cure process, the pressure was held at 45 psi as specified by the manufacturer. The temperature was increased to $250 \mathrm{~F}$ at a 
rate of 5 degree $\mathrm{F} / \mathrm{min}$ at a dwell time of 90 minutes. After this time period, the temperature was cooled to $140 \mathrm{~F}$ at the same rate as the heating. At this point, the pressure was released and the part removed from the autoclave. Vacuum bagging materials were discarded and the parts removed from their molds. The plot for the Cycom material is shown in Fig.( 3.2) for reference.

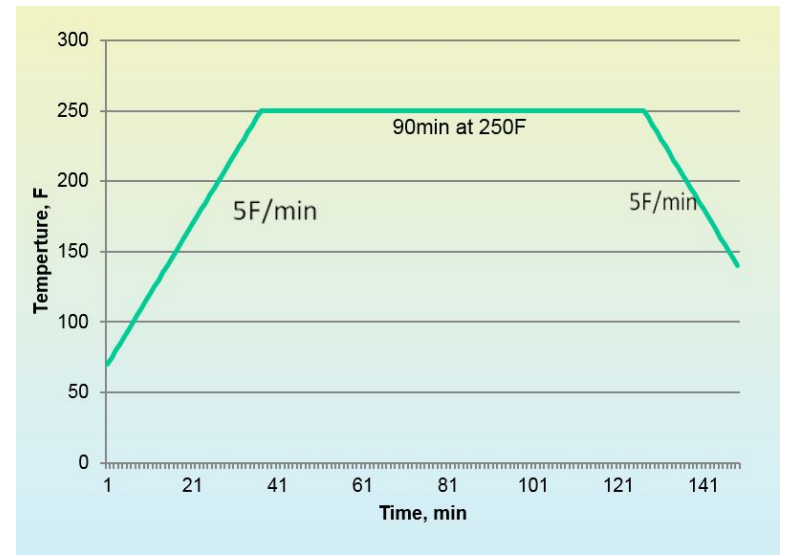

Figure 3.2: Cycom cure cycle

\subsection{Wing Mold Design and Manufacturing}

In this section, I will cover the design of all the molds. Multiple molds for the skin were made to determine which would be optimal. The optimal material for composites molds is aluminum, although it can be expensive. For this reason, materials such as medium density fiberboard, high density foam, and thin aluminum sheet were used. Molds for the spars that would span the wing length were also made. The molds were designed in SolidWorks, taking into account the thicknesses of the carbon fiber and vacuuming bagging materials. This would insure that the parts would be the dimensions that were desired.

\subsubsection{Female Mold}

It was discovered that the DFab lab had a CNC machine that could be used to cut wood and foam materials. A 0.75 inch thick medium density fiberboard 
(MDF) from Home Depot was bought and then Gorilla wood glue was used to bond together the multiple layers. After applying the glue and stacking the required amount of layers, weights were placed on top to evenly distribute the glue. Now that the mold stock is ready for cutting, the female mold was designed in SolidWorks software so that the half of the wing profile would be cut into the block. The SolidWorks representation of the mold is shown in Fig.( 3.3). Since the airfoil is symmetrical only one half of the mold needed to be made. Two identical skins would make up the skin and bonded at the leading and trailing edge during wing assembly.

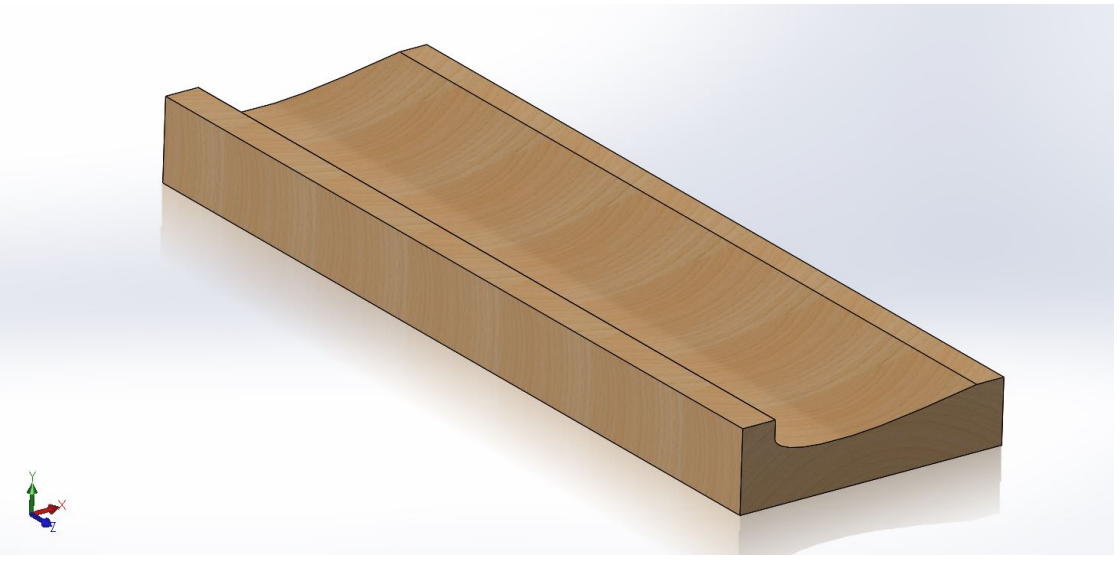

Figure 3.3: 3D model of female mold

Once the mold was finalized in SolidWorks, it was then imported into the RhinoCAM software on the computers at the dFab lab. This software would be used to design the pathing for the tools that would be used to cut the mold stock.

Once the pathing for the mold was done and approved by one of the technicians we then scheduled an appointment to come in and cut our part. With the help of a technician, the mold stock was screwed down onto the table in the right orientation about the origin. Then the RhinoCAM file was imported into the Haas $\mathrm{CNC}$ machine with the use of a flash drive. Before starting the machine we put on respirators to minimize the amount of dust and fumes that would enter our bodies. Once ready the start button on the machine was pressed and it immediately began 
following the specified paths designed in the software.

In order to keep the cutting area clear of wood shavings, compressed air was used to blow away excess debris. This was important in order to maintain a smooth finish throughout the cutting process. The female mold can be seen in Fig.( 3.4) halfway through the cutting process.

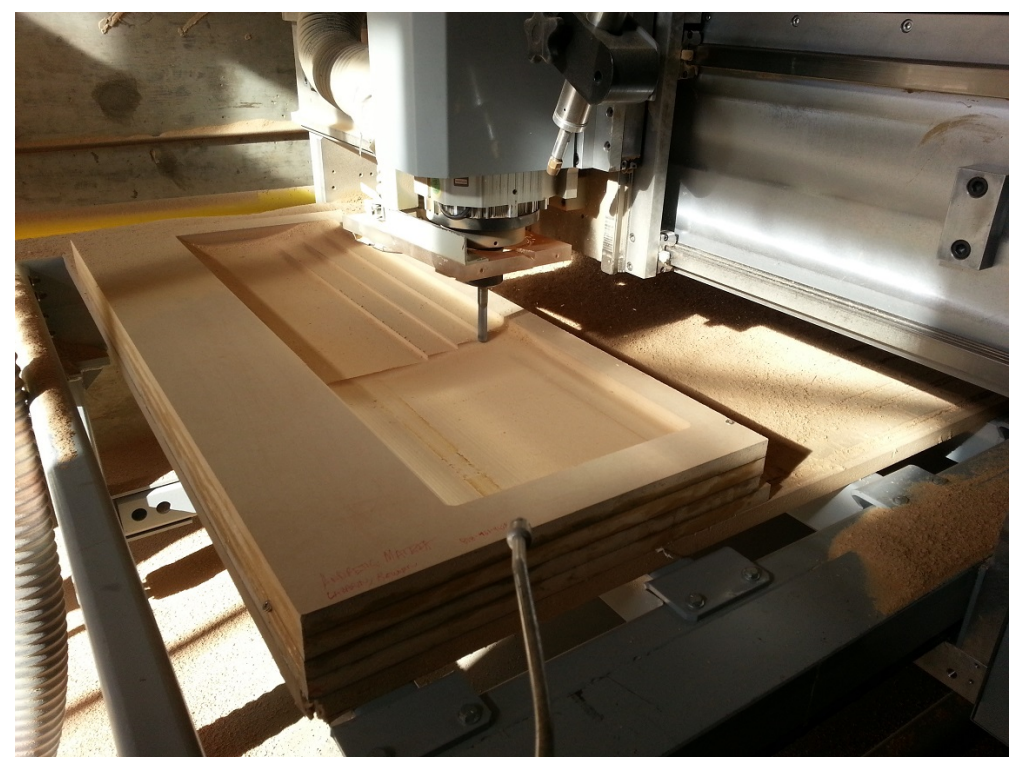

Figure 3.4: Machining of female wing skin mold in progress

Two cutting tools were used for the machining of the mold; one that was larger and a smaller one for finishing. When the $\mathrm{CNC}$ was done cutting with the first tool, it would return to its original position. Then, the finishing tool could be installed to provide a smooth finish. Next, the area was cleared for safety and the start button was pressed again to continue the machining. Once the CNC finished machining the mold the area was cleaned of all dust and the mold was removed from the table. The finished female mold can be seen in Fig.( 3.5) ready to be used for a skin layup.

Since the mold was made out of wood, the surface would need to be sealed because of its porosity. This would require a sealer called Duratech, which was not available to us and costly. Our solution was to use non-porous Teflon material 
or vacuum material between the carbon fiber and the mold. This solution allowed wing skin parts to be made in the autoclave, although there was concern with having a discontinuity at the leading edge in the final wing assembly.

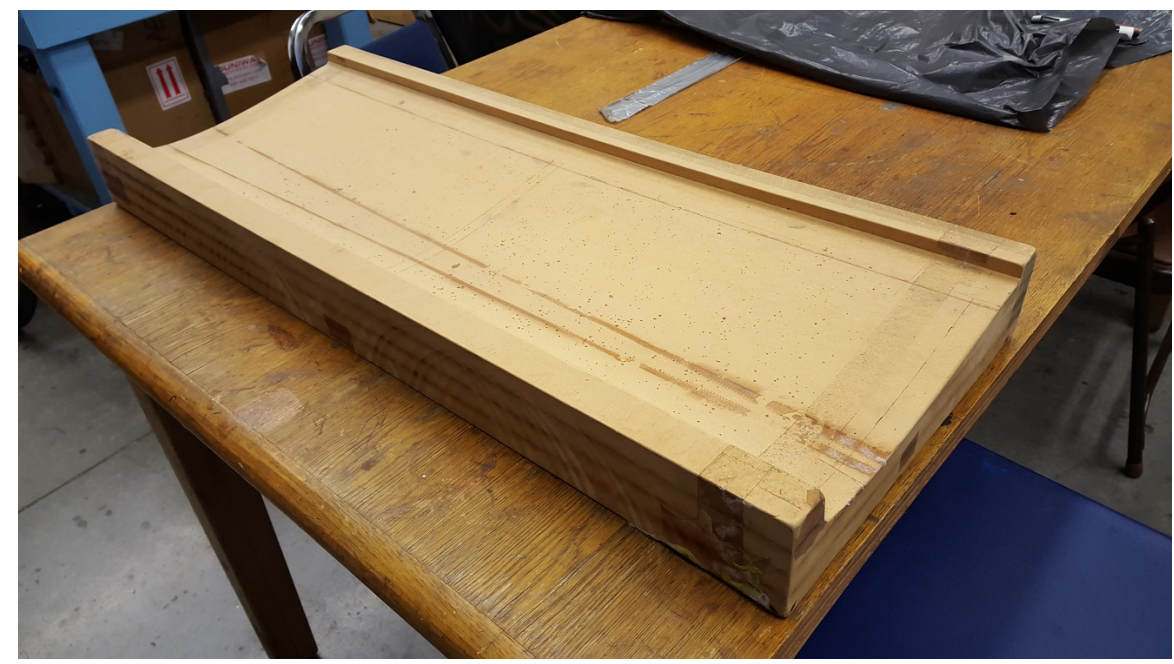

Figure 3.5: Finished female mold ready to be used for skin layup

\subsubsection{Male Mold}

In order to eliminate the problems that would occur from trying to bond the leading edge together between the two halves of the wing skin, a male mold was made. The male mold would be shaped like an airfoil so that uncured carbon fiber would be wrapped around it to form the wing skin. It was machined using a Haas CNC machine that is located in the Cal Poly Aerospace Water Tunnel in building 41.

The mold stock used was longer than the airfoil shape so that flat sides would remain on either side for stability. Once one half of the airfoil was machined, the stock would be flipped to machine the other side. Since the airfoil shape is symmetrical, the same program was used to machine the second half of the mold. Afterwards, the supports at either end were cut leaving an airfoil shaped mold.

Since the foam that the mold is made out of is porous, it does not provide a good surface to lay carbon fiber onto. Non-porous material would have to be put 
around the mold in order for the carbon fiber not to adhere to it. This makes the part susceptible to wrinkles from the non-porous material when vacuum bagging the part. To eliminate this problem, flexible aluminum sheet with a thickness of $1 / 16$ " was bonded to the foam mold with the use of a two part magnolia structural adhesive. Care was taken to measure out the specified amount of part A and B, which is 100:77 respectively, by mass. Both parts of the adhesive were weighed with the use of the scale inside the fume hood to allow ventilation. Next, the measured amounts of each part were mixed together with the use of a popsicle stick for a few minutes until the mixture was made homogeneous.

Then, it was placed on one half of the wing mold and spread evenly using a flexible plastic spreader. A metal sheet big enough to cover half of the mold was carefully placed onto the adhesive. In order to force the metal sheet to take the shape of the mold, it was vacuum bagged onto the heat table in the Aerospace Composites Lab. The mold along with the metal sheet can be seen in Fig.( 3.6), as well as how the mold was vacuum bagged to the heat table. The heat table was turned on to accelerate the curing process, but we found that the heat did not travel through the foam since it is meant to be for insulation. The mold was left under vacuum overnight for the adhesive to set. Then, the other side could be glued on.

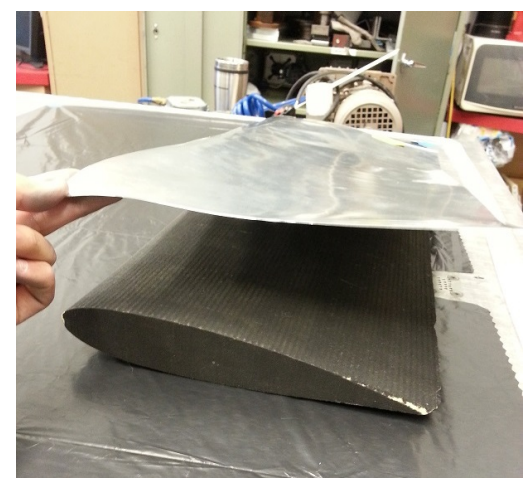

(a) Metal sheet on top of the foam male mold

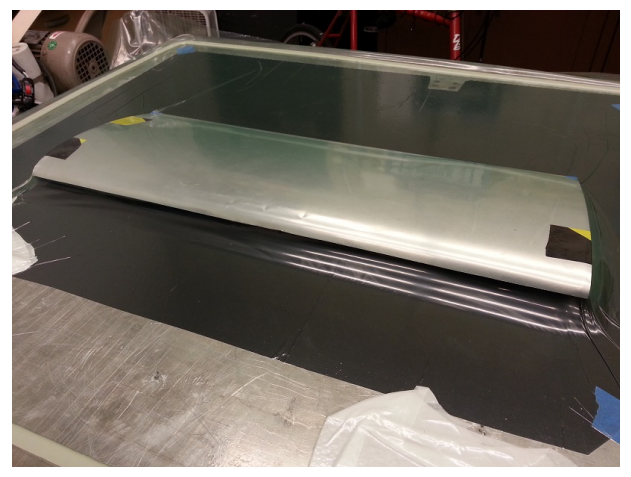

(b) Aluminum sheet vacuum bagged to the foam with the use of a pump

Figure 3.6: Bonding of aluminum sheet onto the porous foam mold 
The same procedure was used to glue the second side, but when we examined the mold after drying, we found that the initial side had delaminated. Since the mold could not rest flat onto the table, we presume that the pressure of the vacuum bag slightly deformed the mold. The bending made the aluminum sheet bubble where it dis-bonded from the foam. In order to fix the mold, a razor was used to cut across the delaminated section and bonded a new piece of sheet metal onto the exposed area. In order to insure a smooth transition between the two metal sheets extra adhesive was used in this discontinuity. Then the adhesive was sanded down to provide a smooth area between the two difference aluminum sheets. Extra release agent was used in this area when curing the wing skin to insure debonding.

What could have eliminated this problem was to use an envelope type vacuum bag when gluing the second sheet. By doing this, the pressure would be evenly distributed on both sides of the mold and no bending would occur.

At the point where the two edges of the sheet metal meet, there was excesses adhesive that would have to be sanded down using 150 grit sandpaper. After this area was smoothed out as best as possible, it would then be ready for a carbon fiber layup. Fig.( 3.7) shows the finished male mold along with two carbon sheets ready to be cured to the shape of the wing skin. This mold was ultimately used for the final wing models since it allowed the skin to be a single part.

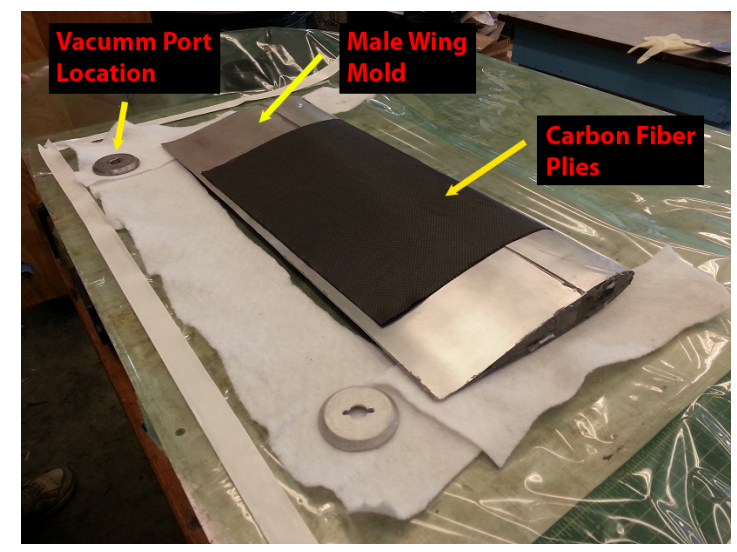

Figure 3.7: Finished female mold ready to be used for skin layup 


\subsubsection{Leading Edge Spar Mold}

In order to make the carbon $\mathrm{C}$ - channel spars, a mold would have to be made that could withstand the heat of the autoclave. The mold is relatively simple due to the simple shape of the spars. It was decided to use MDF for this mold, since its is low cost and easy to cut and shape. The length of the mold was made to be 34" so that two spar layups could be cured at the same time on one mold. The cross-sectional dimensions of the leading edge spar can be seen below in Fig.( 3.8). The angle cut was done by rotating the blade on the saw and then making cuts on the top and bottom of the MDF.

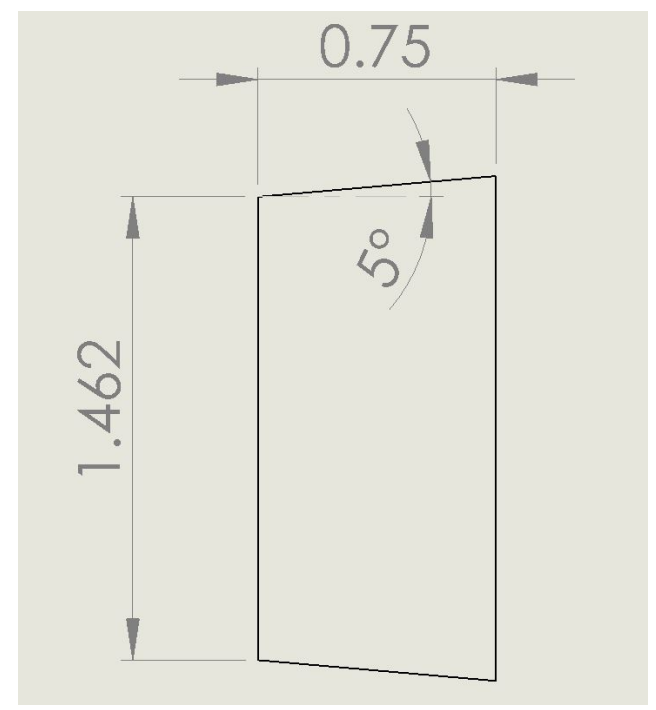

Figure 3.8: Cross-sectional dimensions of the leading edge spar

After the molds were cut to the right size, scotch tape was applied to provide a smooth surface for the carbon fiber layup. To provide even better release, Chemtrend release agent was used on the surface of the tape before the pre-impregnated carbon fiber was laid on the mold. This provided a slick surface so that the cured carbon fiber would not adhere to the mold. The instructions for the release agent were followed from the manual given by the manufacture.[15] The agent was poured onto a paper towel and then wiped on the surface of the 
mold. Then, a dry paper towel was used to wipe off the excess. It is recommend to apply at least 4 layers of the release with 15 minutes between each coat, so that the release agent has time to dry. After the last layer was applied, the agent was allowed to dry for about 45 minutes before the carbon fiber was laid on top of it.

\subsubsection{Trailing Edge Spar Mold}

A similar procedure was conducted to make the trailing edge mold including the taper on the two faces. Once again, this was done so that the flanges on the $\mathrm{C}$ - channel would follow the contour of the wing skin. This would provide a better bonding area between the spars and the wing skin. The dimensions of the trailing edge mold can be seen below in Fig.( 3.9).

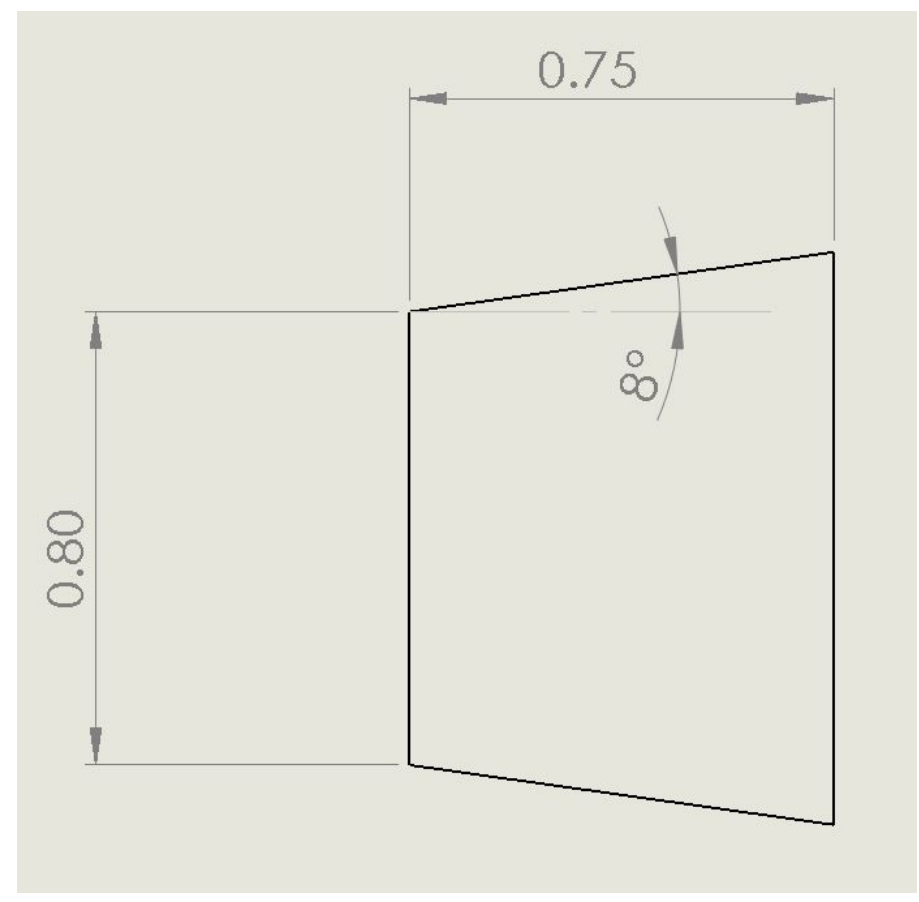

Figure 3.9: Cross-sectional dimensions of the trailing edge spar

\subsection{Manufacturing of the Wing Skin}

In order to eliminate possible problems with bonding the two halves of a wing skin together, it was decided to use a one piece skin part. To do this the male 
mold was used and the carbon fiber weave was wrapped around the mold in order to take its shape.

In order to prepare the mold for the layup, 4 layers of Chemtrend sealer were applied at 15 minutes intervals so that each layer would dry before the other. The sealer would fill in any holes present in the mold so that the excess epoxy from the pre-impregnated carbon fiber would not seep into them. After the sealer was applied, a Chemtrend release agent was used in order to provide a smooth slick surface in order to ease demolding. It is recommended to use about $4-6$ layers of release agent initially, following the same instructions explained above for the sealer. Since the release agent does not get removed when removing the finished part, subsequent layups only require adding 2 or 3 layers of release to the mold.

The plies for the wing skin were cut to be 19.5" long and 16" wide in order to wrap around the mold by about 0.5 " away from the trailing edge. Not bonding the trailing edge together would allow it to be removed from the mold by pulling the two halves apart. On top of the aluminum surface, two layers of carbon fiber weave were laid down and care was taken to remove as many air bubbles as possible. The layers on the wing mold and two vacuum ports are pictured in Fig.( 3.10a). On top of the carbon fiber, a non-porous Teflon sheet is placed in order to keep the cloth breather on top from adhering to the carbon fiber. The cloth breather allowed air to pass through causing greater pressure on the part.

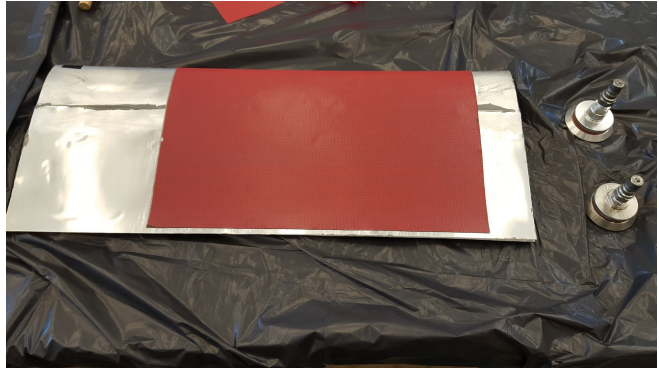

(a) Carbon fiber plies on wing skin mold

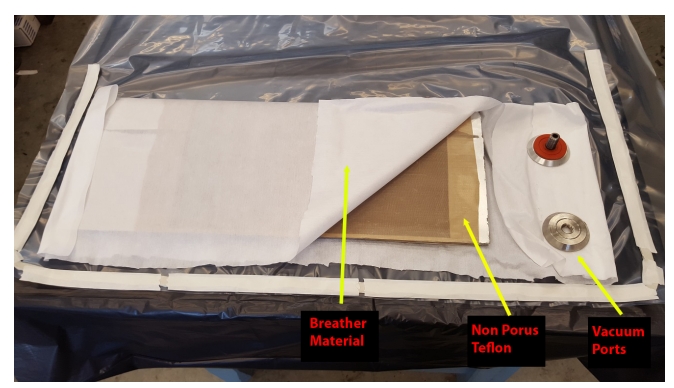

(b) Wing skin vacuum bag layup

Figure 3.10: Wing skin layup 
Now, the wing is ready to be put into an envelope type vacuum bag with two air valves that would be used for the autoclave and external pump pressure. The finished layup under vacuum with the two valves can be seen below in Fig.( 3.11a) while debulking. The finished part after curing the carbon fiber according to the manufactures specifications can be seen in Fig.( 3.11b). For assembly, the inside bonding area of the skin was abraded with 180 grit sandpaper.

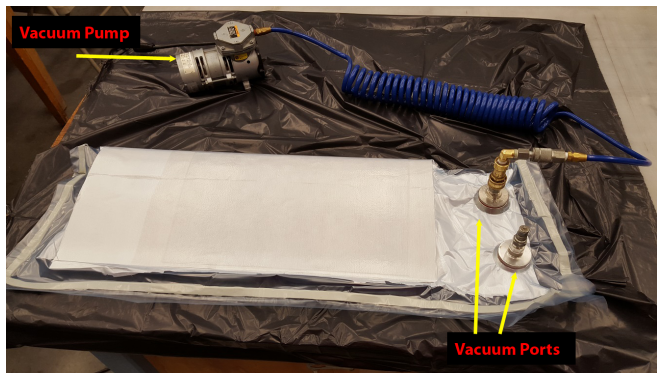

(a) Wing skin layup under vacuum

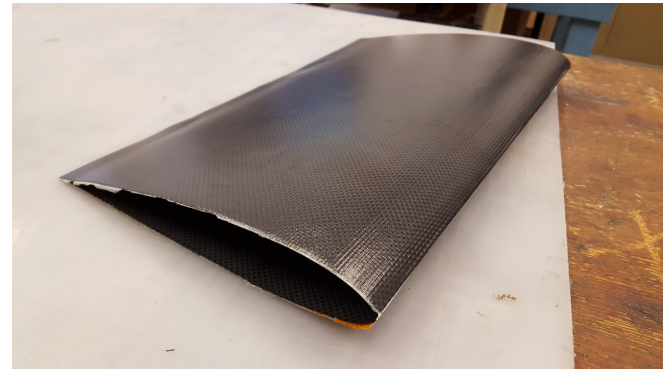

(b) Cured wing skin

Figure 3.11: Wing skin layup

\subsection{Manufacturing of the Spars}

After the spar molds were prepared with the release agent, they would be ready for carbon fiber to be laid onto it. The carbon fiber plies were cut so that they would have a flange length of 0.5 inches. This would make it so that at post-cure, little cleaning of the parts would be necessary. Once the fabric was cut to the dimensions required, they were laid on top of the spar molds carefully. Three and two layers of Cycom carbon fiber weave were used for the leading and trailing edge spars respectively. An example of the carbon fiber laid on top of the mold can is pictured in Fig.( 3.12a). Since most of the force would be concentrated over the leading edge, it was made thicker than the trailing edge.

Once the fabric was smoothed out and placed evenly on the molds, it would be ready for vacuuming bagging. In order to reduce warping of the mold, they were vacuumed onto a flat aluminum plate. A layer of release vacuum material was 
placed under the mold to protect the surface from excess adhesive. On top of the carbon fiber, non-porous Teflon material was placed, and then cotton breather, to allow for optimal pressure around the part. Then, the release vacuum bag was placed over the layup and sealed with sealant tape that was put on the metal plate. The spar layup is shown in Fig.( $3.12 \mathrm{~b})$ without the release bag or vacuum on the part.

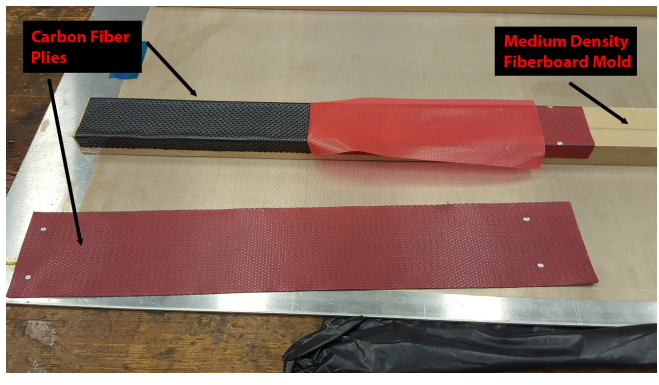

(a) Carbon fiber plys on spar mold

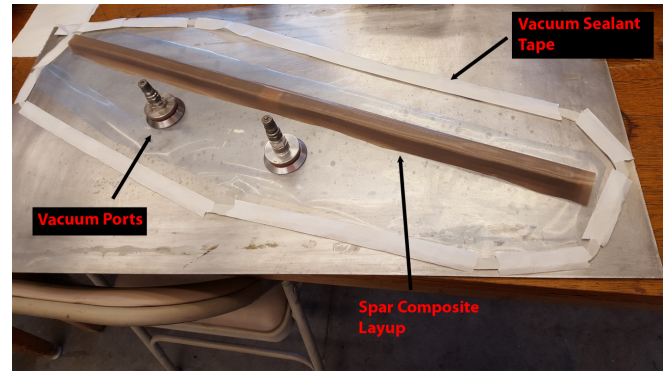

(b) Spar vacuum bag layup

Figure 3.12: Spar layup

After the vacuum was placed on the part, the layup was checked for leaks and let to debulk. Then, the autoclave was prepared and the layup was put into it. The layup was set to cook for the specified cure cycle for the Cycom material. After removing the part from the autoclave, the bagging materials were discarded and then part removed from the mold. Fig.( 3.13) shows the finished leading and trailing edge spars that were used in the wing models. The silver markings were used as a guide for cutting the parts to the desired length. 


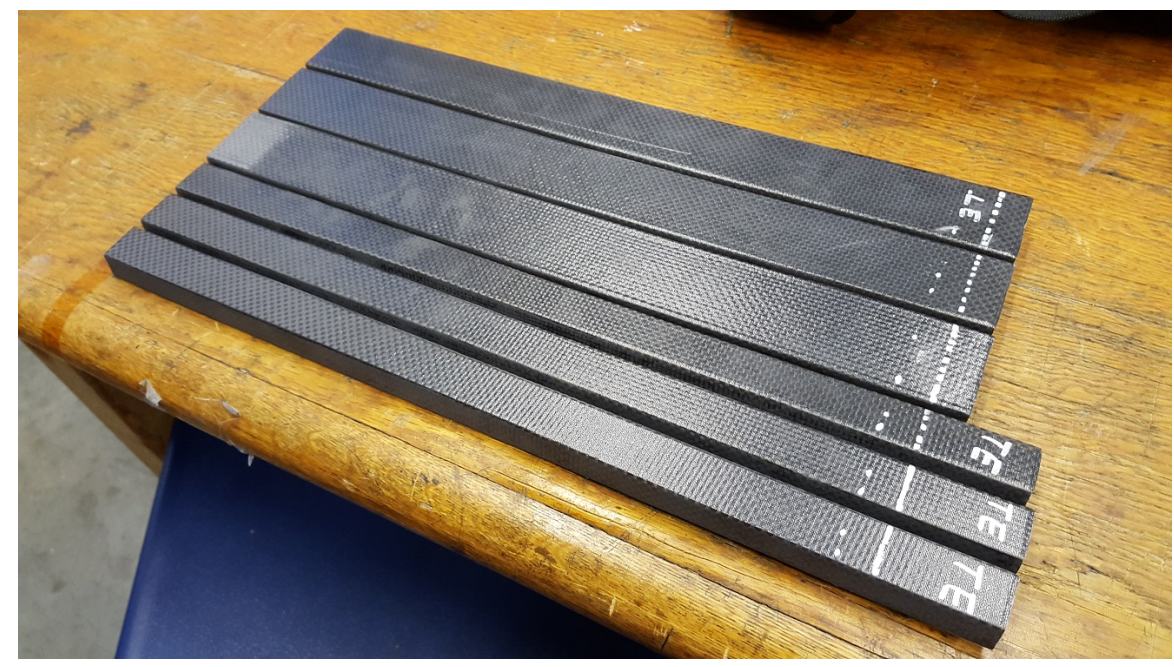

Figure 3.13: Cured leading edge and trailing edge spars

\subsection{Manufacturing of the Ribs}

At the end of the wing model, a rib was placed that would provide holes for mounting onto the fixture, as well as the hinge for the load from the Instron. In order to make the ribs, a laminate was made from 10 layers of Hexcel carbon fiber weave in the heated pressure plate. The plate was cured using the specified cure cycle given from the manufacture. Using the SolidWorks model of the rib, a drawing was made so that it could be printed and glued onto the plate using Elmer's brand glue stick. This type of glue was chosen since it is water washable and can easily be removed once the outline and holes have been cut. An example of the rib template being glued onto the laminate can be seen in Fig.( 3.14 ). Five holes were drilled into each rib so that bolts could be used to mount to the rigid fixture. The hole diameters in the rib were sized to be .145", which includes tolerance for the screw diameter. 


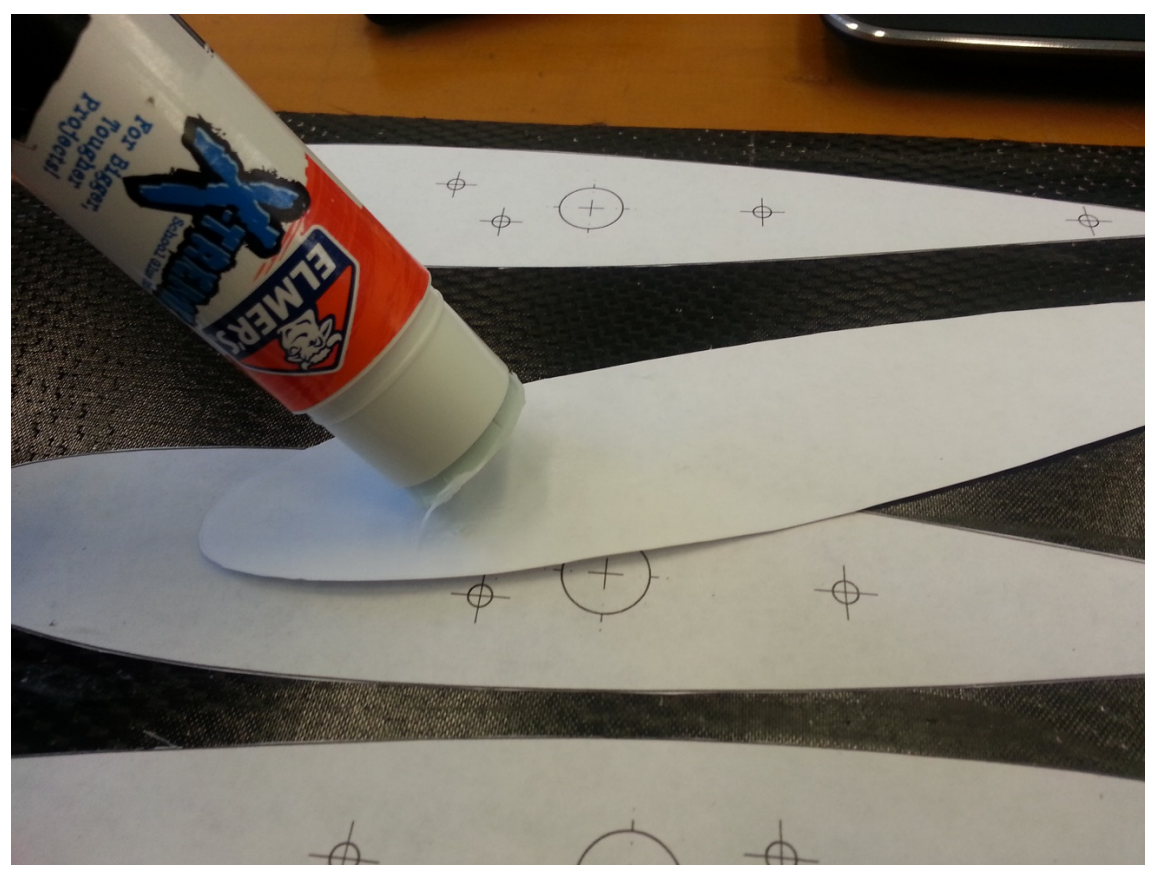

Figure 3.14: Applying templet on carbon fiber plate

After the templates were glued onto the 12 "x 12 " plate, each rib was cut by hand using a vertical band saw. Out of each laminated plate, 8 ribs were able to be cut from it, with enough room between for the cutting blade, which can be seen Fig.( 3.15). By gradually maneuvering the plate into the saw, the outside contour of the ribs were able to be cut close to their desired dimensions. The band saw left a smooth surface, except for some splinters on the bottom layer, which were easily sanded off using 220 grit sandpaper. An example of the rib partially cut from the carbon fiber laminate stock can be seen in Fig.( $3.15 \mathrm{~b})$. 


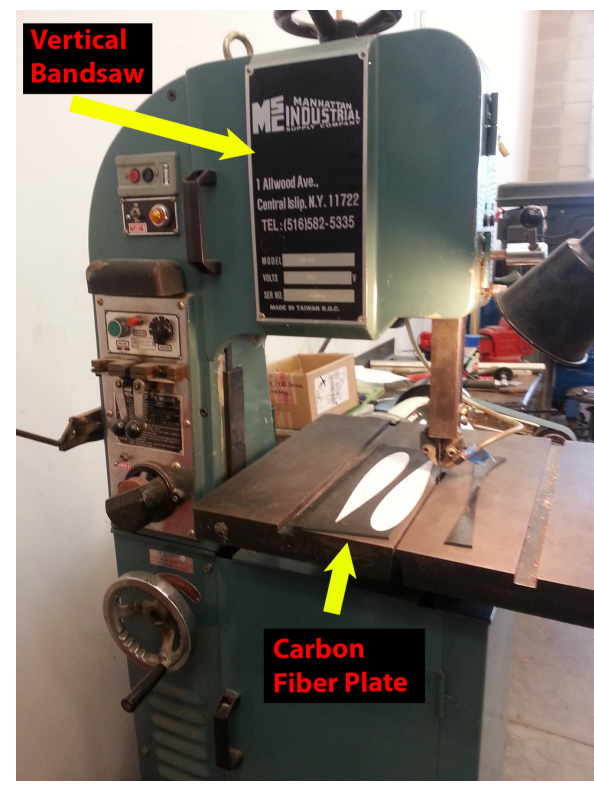

(a) Manhattan Idustrial Supply Co. band saw

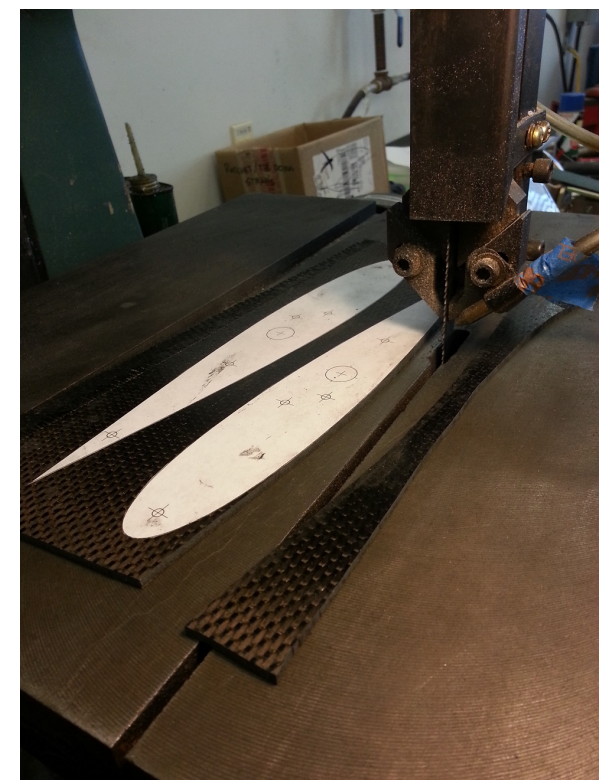

(b) Cutting of rib part

Figure 3.15: Cutting carbon fiber ribs

After the outside profile of the rib was cut, the paper template was kept on in order to locate the holes that need to be cut. The drill press in the composites lab was used to make the holes using steel drill bits, which provided a smooth cut without delaminating the laminate. The final cutout of the carbon fiber rib can be seen in Fig.( 3.16) without the holes drilled out yet. 


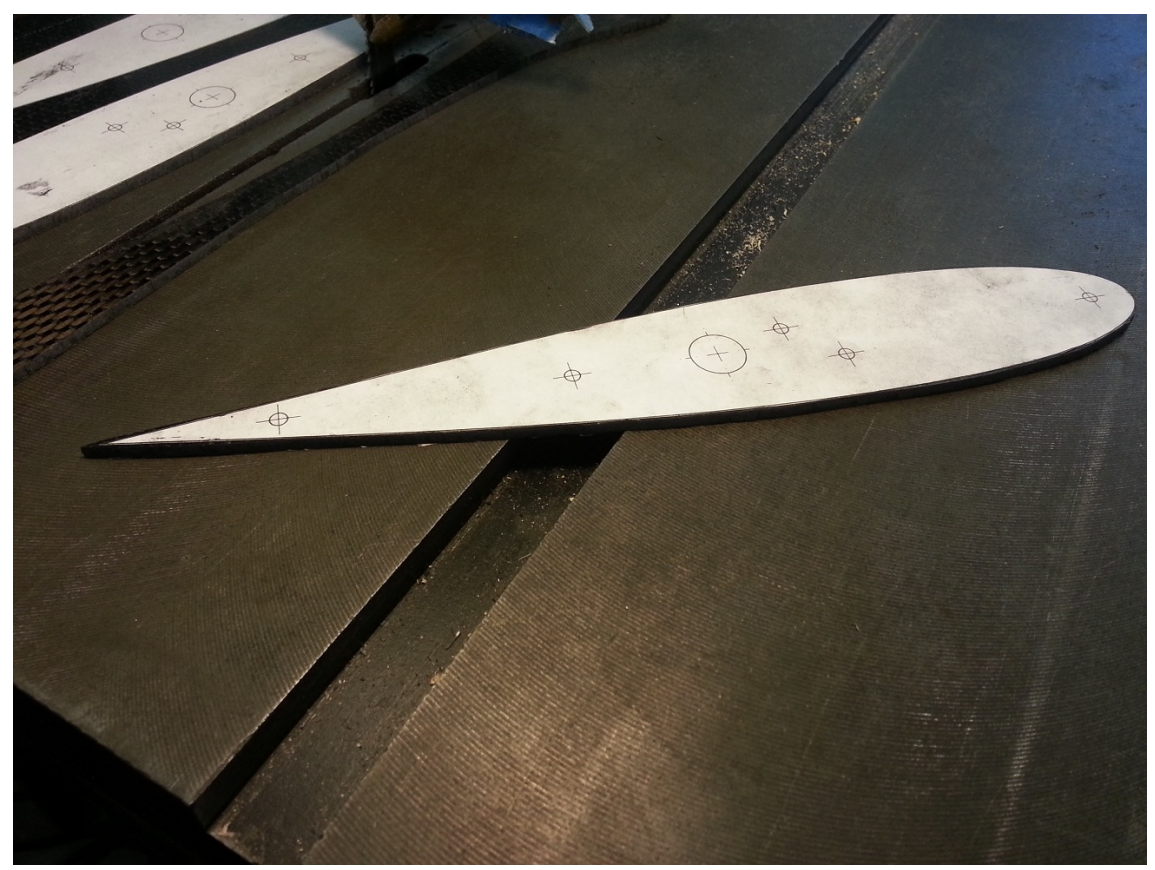

Figure 3.16: Finished rib part

\subsection{Assembly of Wing Specimen}

After all the carbon fiber wing parts were cured, it was time to assemble the wing by bonding joints and surfaces. Throughout the whole assembly process, bonding areas where abraded with 180 grit sandpaper. Adding abrasions greatly increases the bond strength by allowing the adhesive to seep into the cracks. An example of the abrasions can be seen in Fig.( $3.17 \mathrm{a})$. The adhesive was also mixed with chopped carbon fiber, adding to its viscosity, so that it would not move from the bonding area. The chopped fiber also added some rigidity to the joint areas once cured.

The first step was to glue carbon fiber L- brackets to the sides of the spars at the root and tip. Since the spars had such a small surface area, the brackets were used to increase this so that they could be bonded to the face of the rib. First, a layer of release was put down onto the heat table set to $160 \mathrm{~F}$ to expedite the curing process. The adhesive mixture was added to the brackets and pressed 
to both sides of the spar. Small pieces of release film were placed on top of the adhesive to apply pressure and to provide a smooth finish. Then, it was stood up vertically in the manner shown in Fig.( $3.17 b)$ to cure.

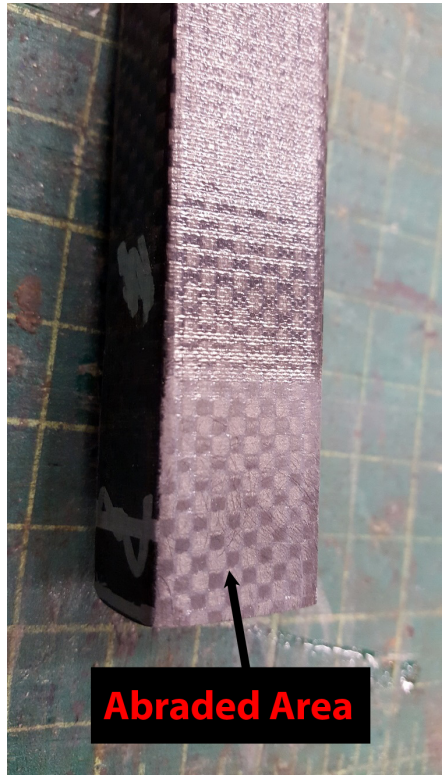

(a) Abraded bonding area on spar

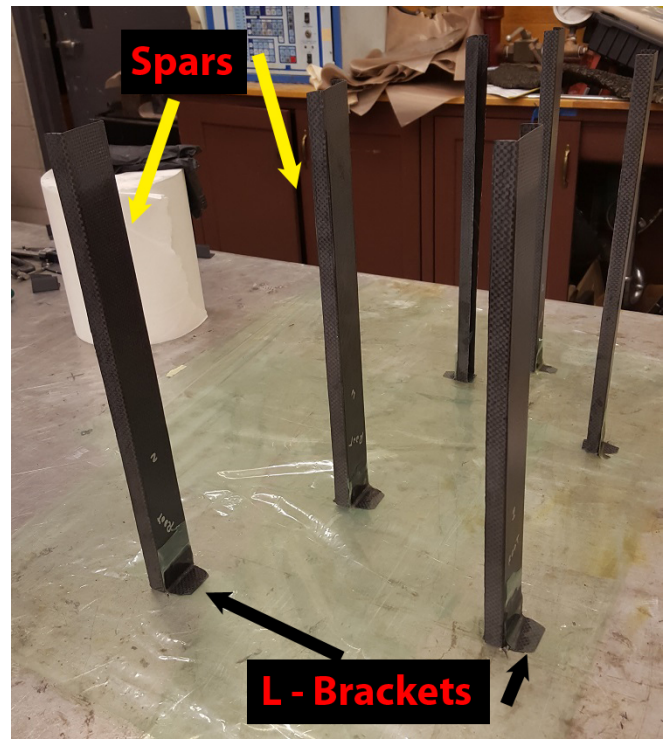

(b) L-brackets being bonded on to spars

Figure 3.17: Spar layup

The next parts that were bonded were the metal $\mathrm{T}$ - nuts onto the carbon fiber ribs. This was the only step where chopped fibers were not mixed into the adhesive, since there was no benefit. In Fig.( 3.18a), a test bond is shown, where a two T nuts are threaded into place with a screw. Adhesive was placed on one of the nuts and the other was used to apply a pressure on the area by torquing the screw. Care was taken to not have extra adhesive seep into the threads, although regardless, the screw would still be able to come out with moderate force. This method was adopted from Richard De Luna's thesis paper and worked just as well for this project as did it for him. All of the of $\mathrm{T}$ - nuts were bonded using the same method and the final bond can be seen in Fig.( 3.18b). Also note in this figure that the abrasions made for the spar brackets were deeper than the others since this was a critical joint. [16] 


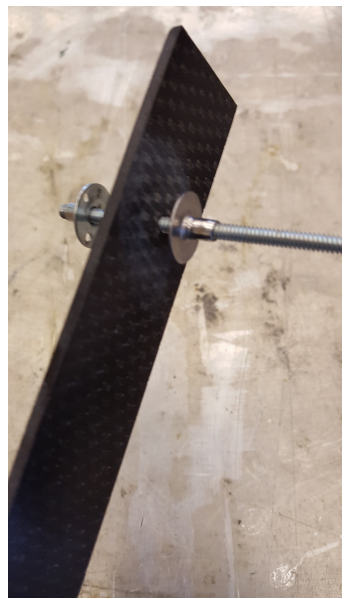

(a) Testing T-nut bonding method

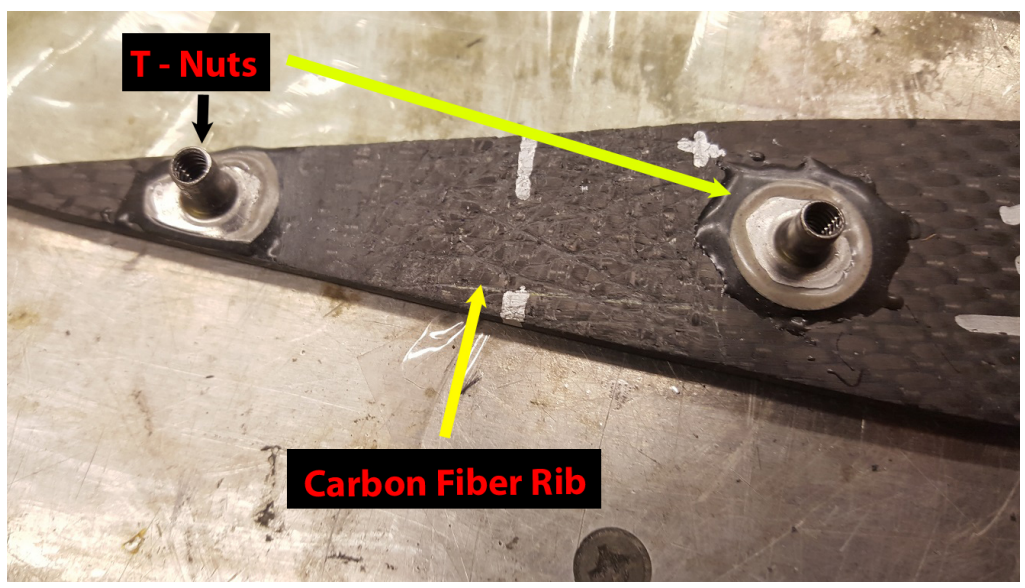

(b) T-nuts bonded onto rib parts

Figure 3.18: Method used to bond t-nuts onto ribs

Once all of the $\mathrm{T}$ - nuts had cured then the trailing edge spars and their brackets were bonded onto the root ribs. This was done by applying adhesive to the surface of the brackets and then pressing it against the rib. Care was taken to align the spars as straight as possible and where the spars were designed to be located. More adhesive was added on top of the brackets to over lap onto the rib to add reinforcement to the joint. Once the trailing edge spars, were cured the same procedure was used for the leading edge spars. The carbon fiber rib with just the trailing edge spar and also with both of the spars can be seen in Fig.( $3.19 \mathrm{a})$ and Fig.( 3.19b). 


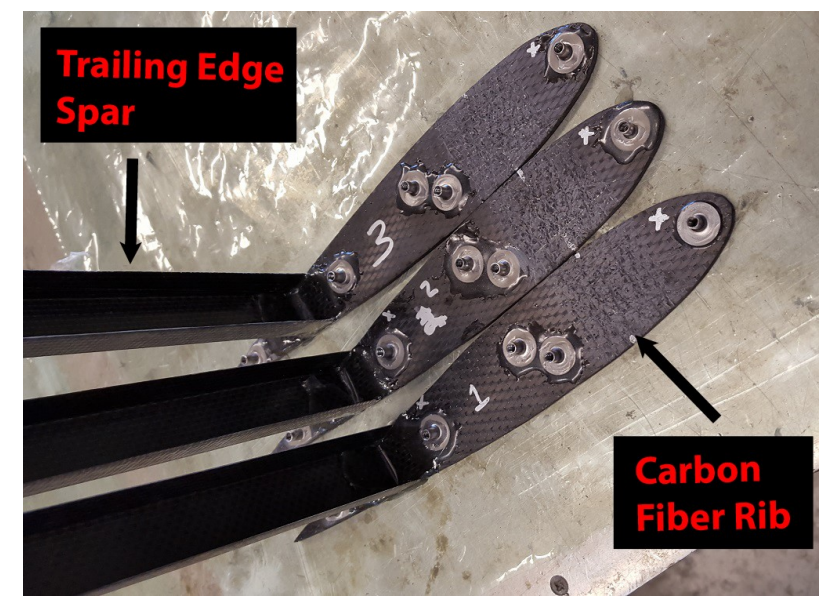

(a) Trailing edge spar bonded onto rib

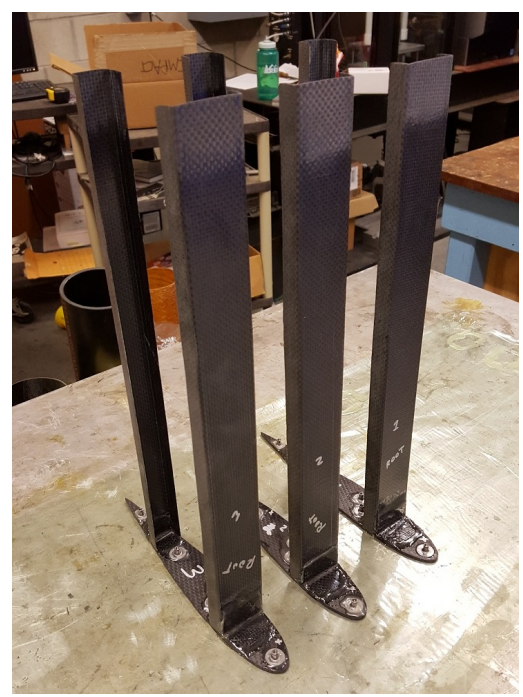

(b) Leading and trailing edge spars bonded on to rib

Figure 3.19: Bonding spars on to first rib

Then, the other half of the ribs intended for the tip would have only the inside L - brackets bonded onto them as shown in Fig.( 3.20). Since the spars glued to the root ribs had an inward angle, they would apply some pressure when bonding to the opposite rib. These brackets simply had adhesive applied to their surface and pressed onto the ribs in their intended location. Then, the outside brackets were pressed on the opposite side so that the joint would be made.

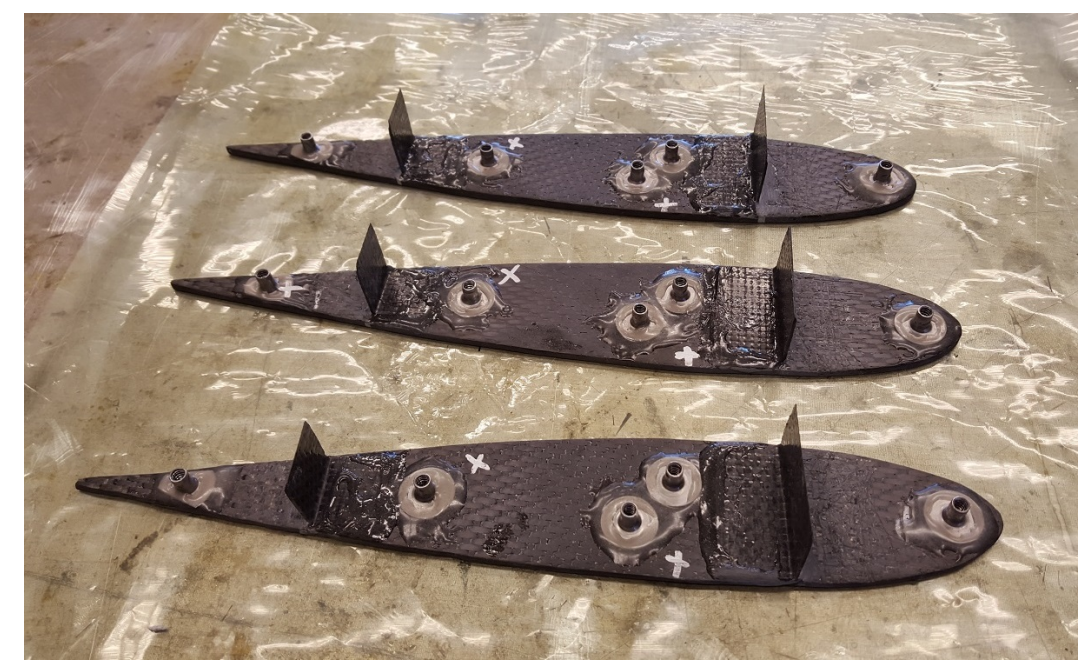

Figure 3.20: Inner brackets bonded to provide pressure when bonding spars 
Fig.( 3.21a) shows the final curing process on the heated table for the internal structure. After this was done curing, the internal structure was cleaned up by sanding any extra adhesive and bonding area. The final internal structure consisting of two spars, two ribs, T nuts and L - brackets is depicted in Fig.( 3.21b). The internal structure was light and stiff, but lacked in torsional stiffness which would be remedied by adding the skin part around it.

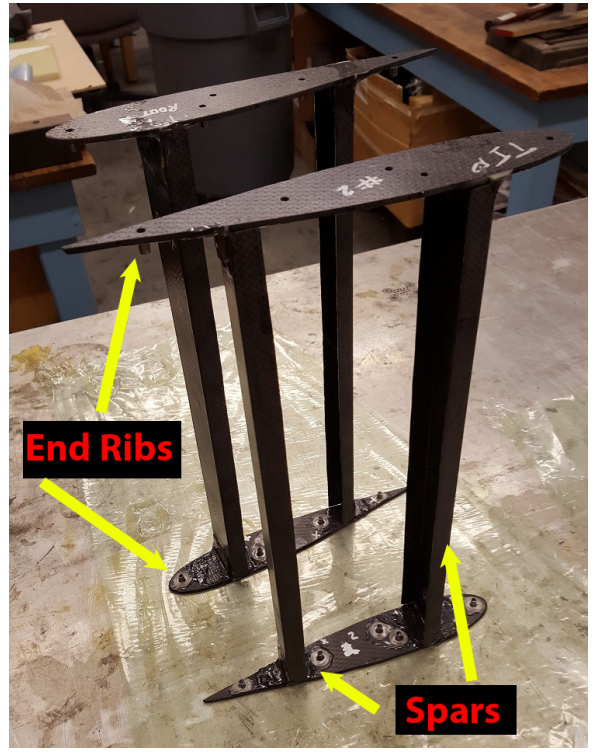

(a) Second rib is placed on spars and bonded

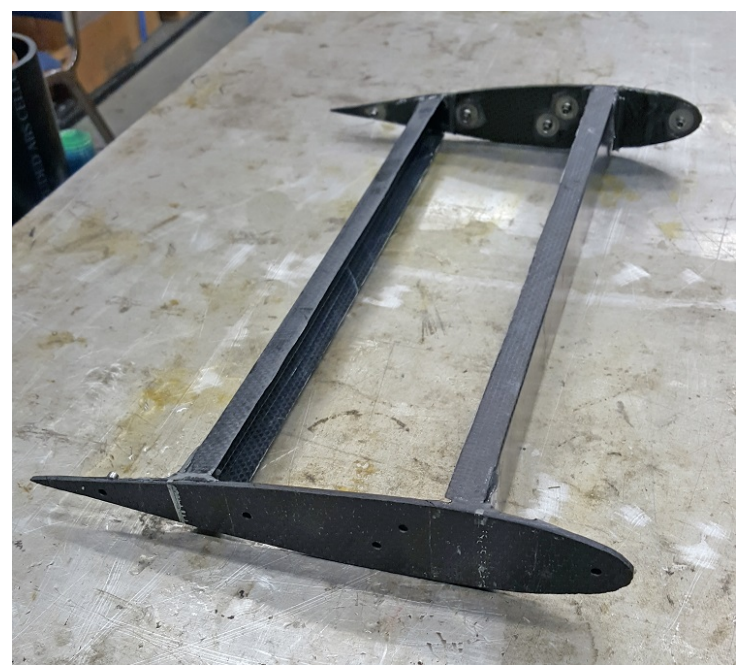

(b) Finished internal structure of wing model

Figure 3.21: Bonding spars on to first rib

Next, the skin was bonded to the internal structure. This was done by adding adhesive to the top and bottom of the spars as well as the ribs. To insure a good bond, extra adhesive was added to the inside of the skin where the internals would be placed. Three screws shown in Fig.( 3.22$)$ were attached to the side of the internals so that they could be used as handles. 


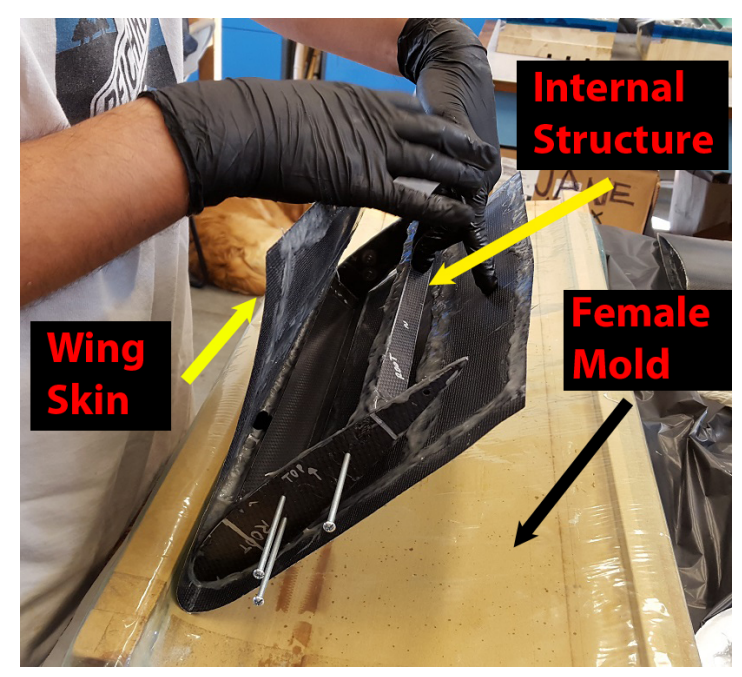

Figure 3.22: Internal structure goes into skin for bonding

In order to apply pressure to the wing skin during bonding to the internals, the assembly was placed into the female mold. Since it had the same dimensions as the current wing, it fit perfectly along its contour on top of release film. In Fig.( 3.23a) the wing was placed in the mold along with the screws used for handling. Another layer of release film was placed on top of the wing and then thick breather cotton that was folded up multiple times followed. The breather was used so that weights could be placed on top of the wing and it would be evenly distributed across the top of the wing skin. This was important so that there would not be gaps in between the skin and internals which could cause delaminations. The weights on top of the curing assembly can be seen in the bottom right in Fig.( 3.23b). Another method to insure the bonds have pressure applied is to vacuum bag the assembly after applying adhesive. This method was not used since adding weights was a straight forward method that was expected to apply sufficient pressure. 


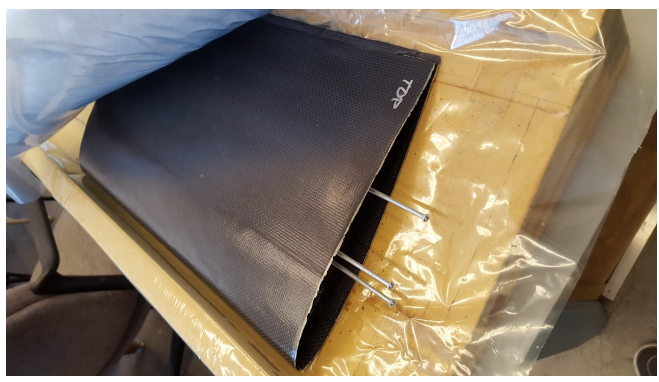

(a) Female skin mold used to hold assembly while curing

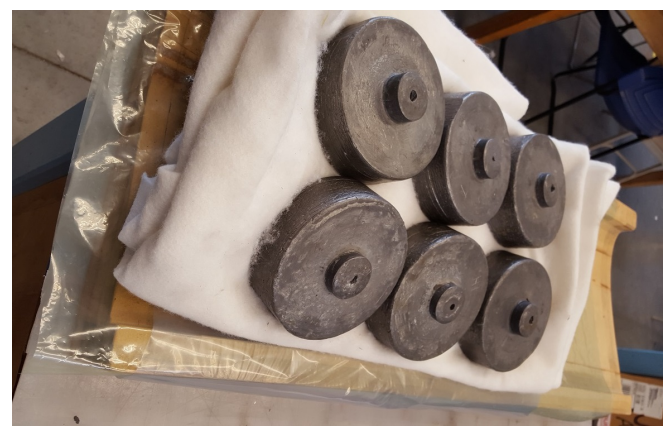

(b) Weights used to apply pressure for bonding

Figure 3.23: Bonding wing skin onto internal structure

After the wing was left to cure over night, the weights and extra materials were removed to uncover the bonded wing model. All that was left after this was to clean up the edges with sandpaper and a Dremel sanding tool and cut the wing to length. The final wing model along with some markings for strain gages can be seen in Fig. ( 3.24).

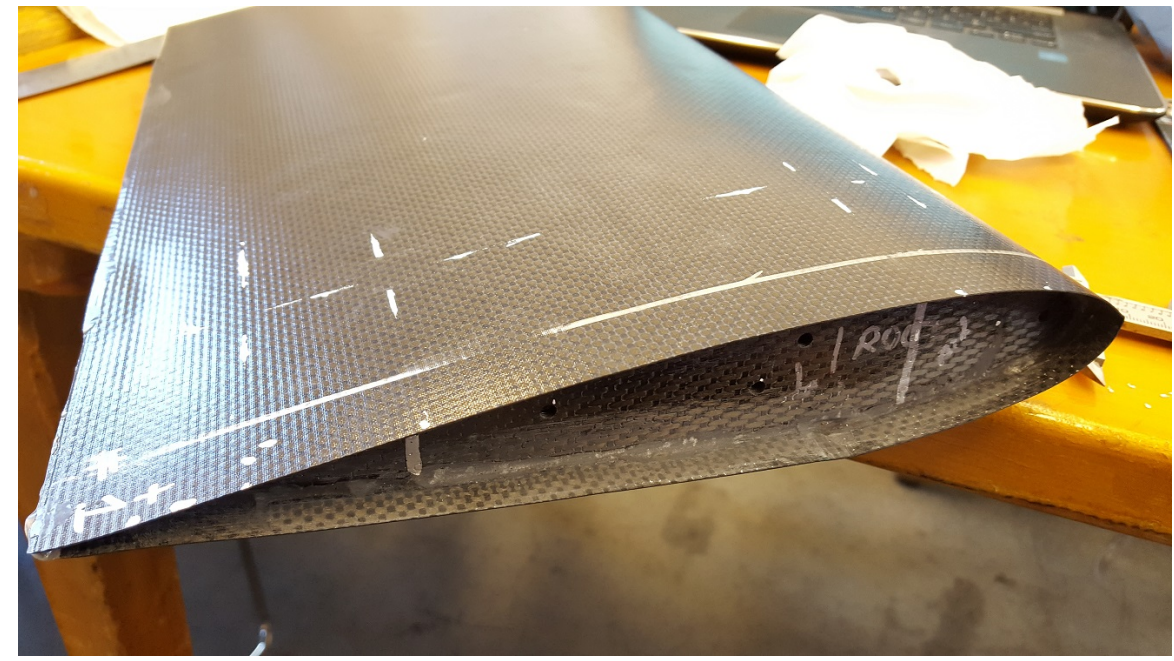

Figure 3.24: Finished wing model after sanding

\subsection{Strain Gage Mounting}

In order to bond the strain gages to the test specimens, the procedures from Vishay Micro Measurements was followed. A summary of their procedures will be 
depicted in this section, although it is not intended to be an instruction manual.

The first thing done was to abrade the bonding surface and to mark where the strain gage will be placed on the specimen. After this was done, an aluminum plate was cleaned so that a strain gage could be placed on top of it. Both of the previous steps can be seen in Fig.( 3.25). Then, a piece of strain gage installation tape from Micro Measurements was placed over the strain gage from one side smoothly. Care was taken to make sure that no air bubbles were trapped under the tape.

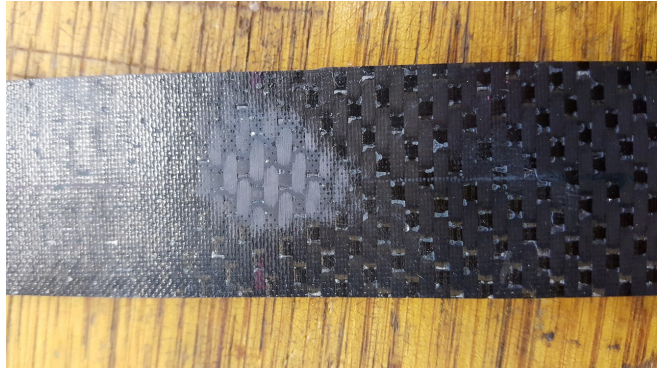

(a) Abraded bonding surface for strain gage on specimen

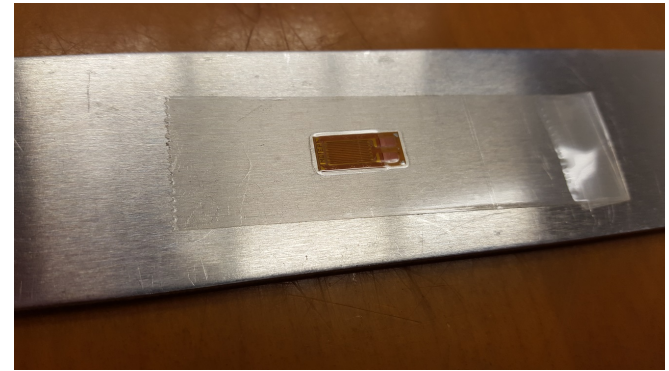

(b) Clean aluminum plate used to attach the strain gage to installation tape

Figure 3.25: Initial strain gage bonding steps

Then, the tape was lifted from one side with a shallow angle to prevent damage to the strain gage. After the tape and strain gage were completely removed from the plate, it was placed where the strain data is needed on the test specimen. Then, one side of the tape was lifted as before until .5 inch of tape was between the strain gage and the specimen surface. Then, catalyst from the M Bond 200 adhesive kit from Micro Measurements was put on the bottom of the strain gage in one smooth wipe. A rosette in this folded back position can be seen in Fig.( 3.26). 


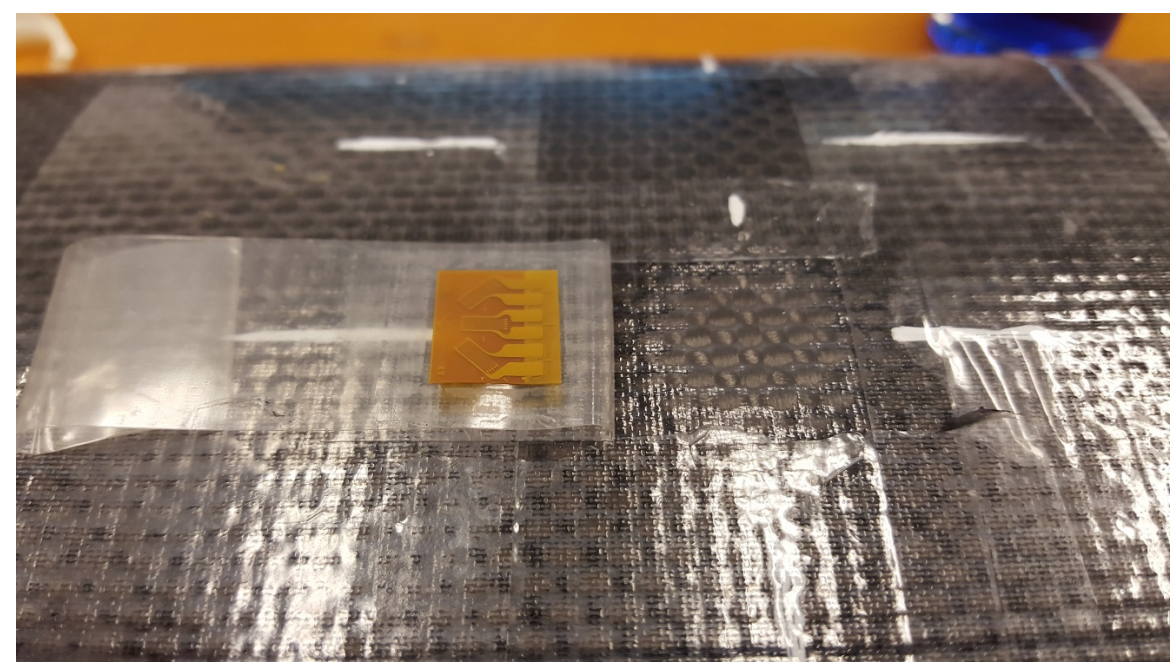

Figure 3.26: Rosette strain gage ready for catalyst to be applied to it

The application brush was wiped on the edge of the bottle 10 times so that there would not be excess catalyst on the strain gage. Having too much catalyst could lead to an uneven bond surface. After waiting one minute for the catalyst to dry, one drop of adhesive from the kit was placed at the edge of the tape. Carefully, the tape and strain gage were pressed down onto the specimen surface with the use of a folded gauze pad. An example of the strain gage and tape ready to be smoothed down onto its bond location can be seen in Fig.( 3.27 ).

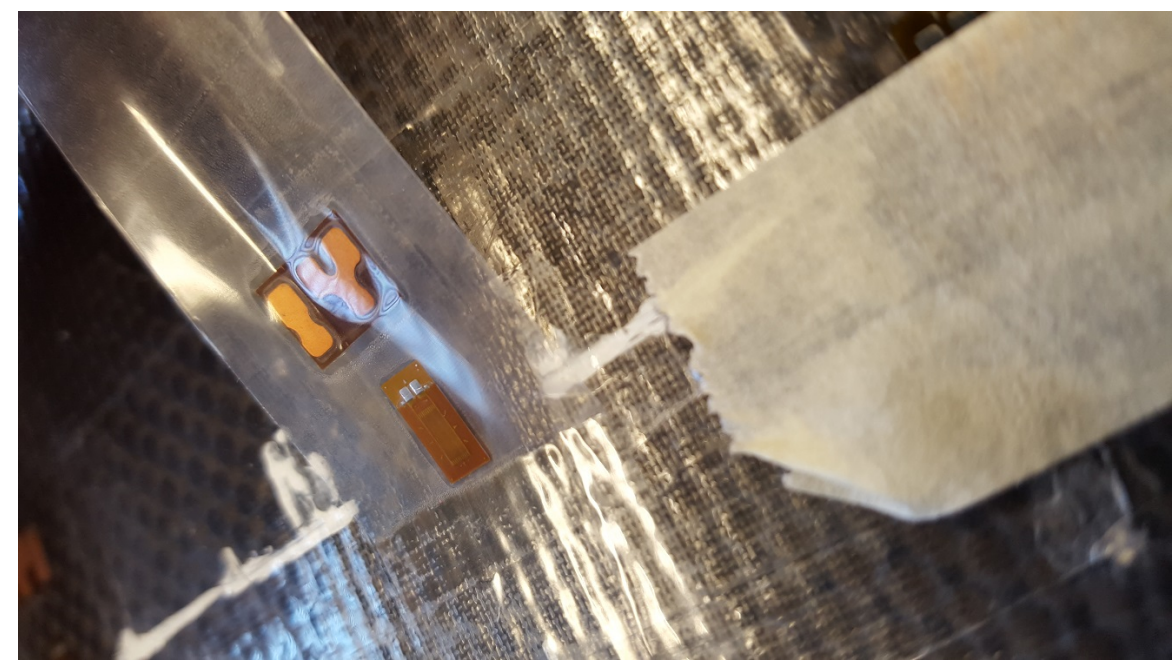

Figure 3.27: Uni-directional strain gage and terminal ready to be applied to bond surface 
Immediately after this, thumb pressure was placed on the strain gage for one minute. After a few minutes, the tape could be removed leaving the bonded strain gage on the specimen. The bonded strain gage and terminal can be seen in Fig.( 3.28) below.

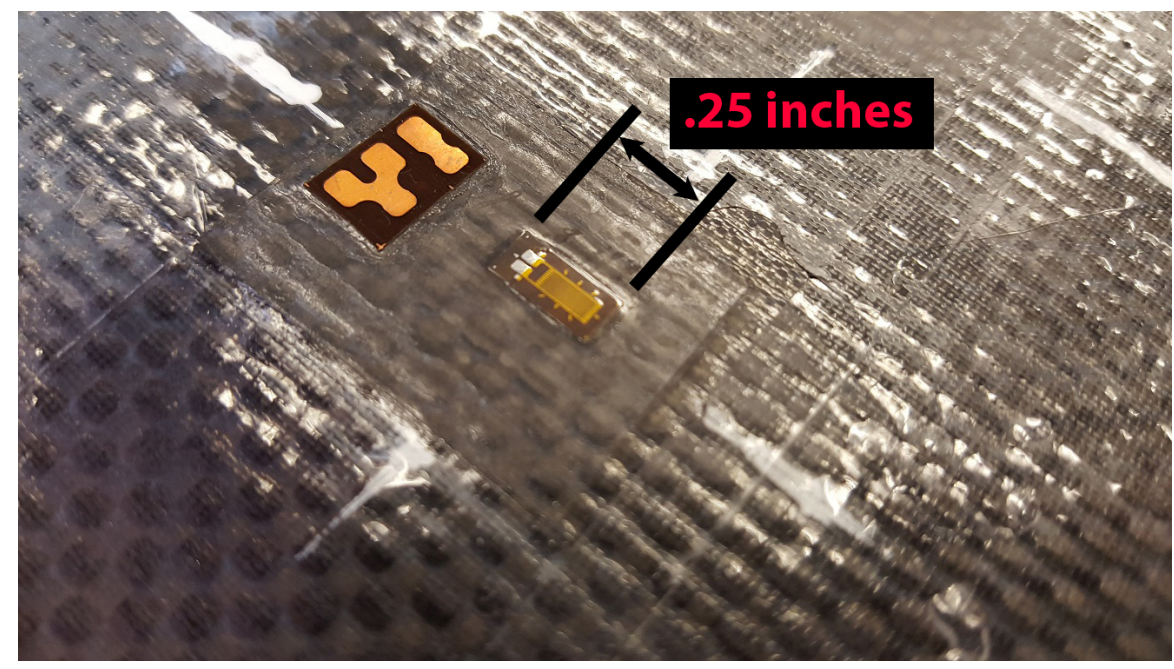

Figure 3.28: Bonded uni-directional strain gage and its terminal pad on specimen

The next steps would be to solder the wires onto the terminals and strain gage tabs. The terminals were added to provide extra security in case one of the lead wires was accidentally pulled enough to break the solder point. With the terminal on there, the strain gage would not be at total loss if this were to occur. The terminals also provide a larger surface for the lead wires to solder onto. It would be difficult to solder straight onto the tiny tabs on the strain gage. Micro measurements masking tape was placed onto of the strain gage in order to protect it from the soldering iron heat as shown in Fig.( 3.29). It was still recommended not to dwell too long on the tab when soldering close to the strain gage. 


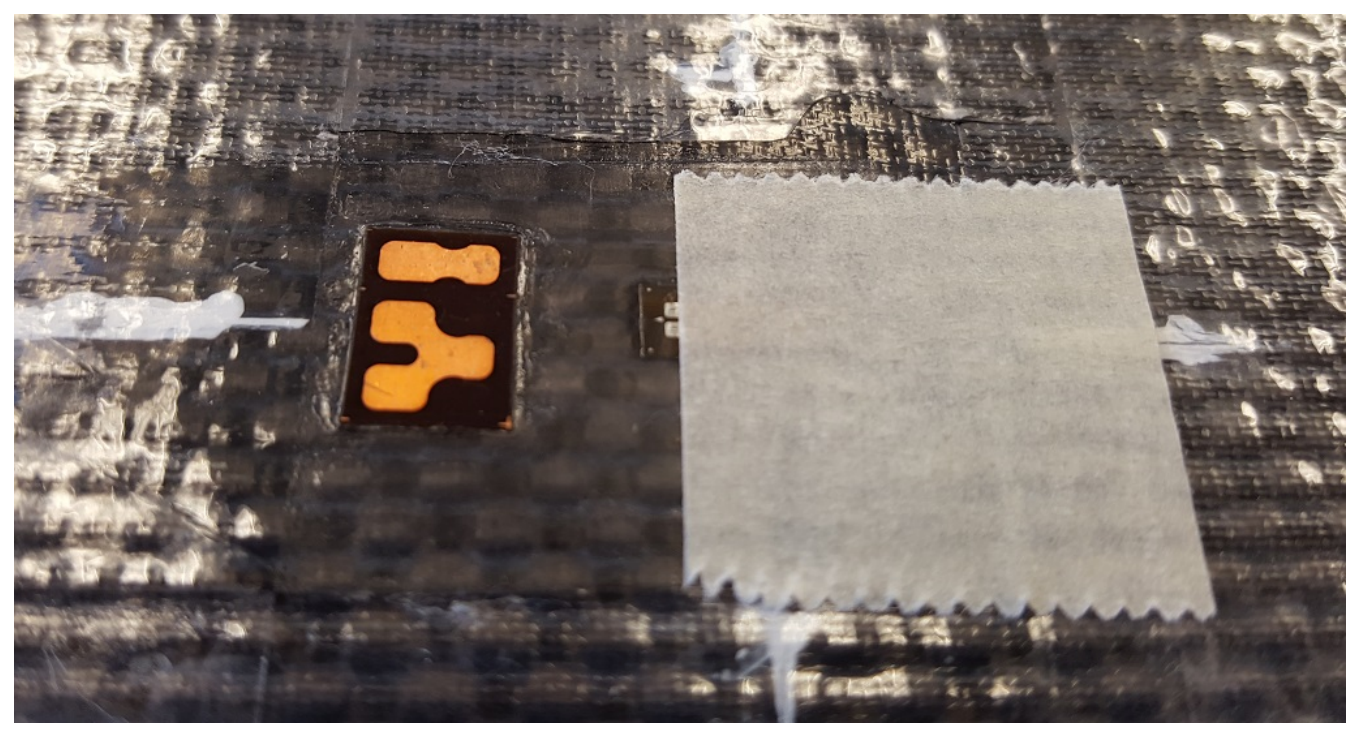

Figure 3.29: Strain gage covered by tape to protect it from heat during soldering

In Fig.( 3.30), the strain gage and terminals are shown with solder pads on their tabs ready for wires to added. A thin wire was used to connect the strain gage tabs to the terminals tabs. Care was taken so that the bridge wires were not in tension, or this could cause them to break when the specimen is loaded. After the lead wires were soldered, the 3 wire cable was soldered to the 3 pronged terminal. As explained in chapter 4 section 2.1, the 3 wire configuration was used to eliminate the lead wire resistance and to limit the strain effects of temperature which are not of interest in this thesis.

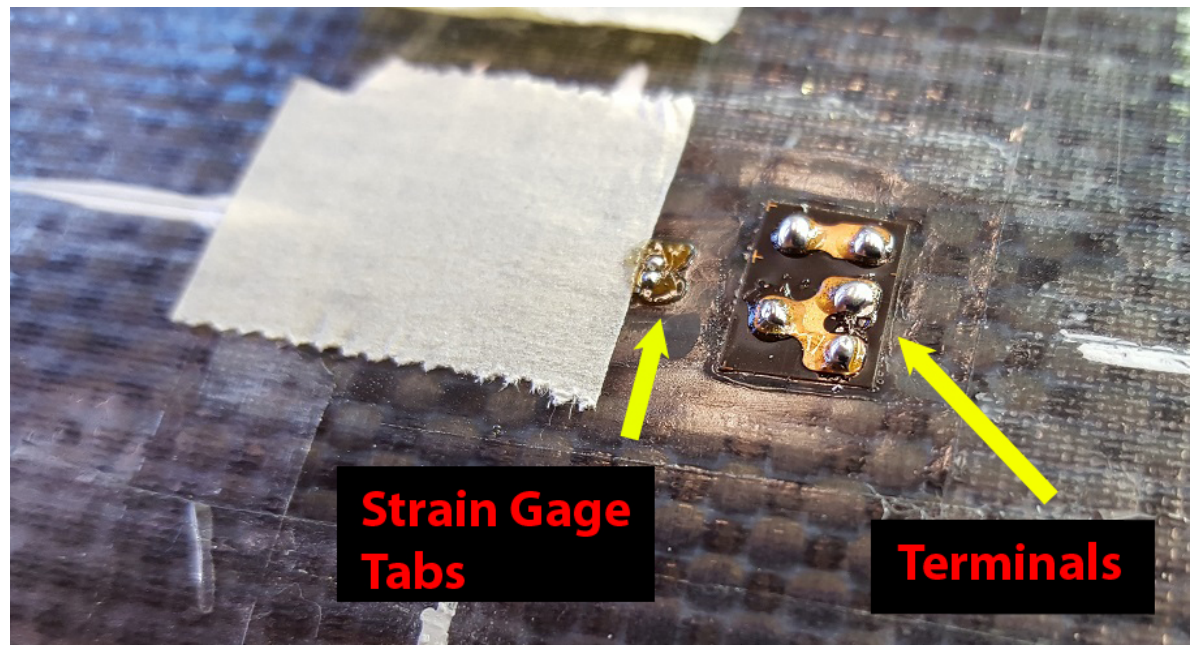

Figure 3.30: Solder added to the terminals and strain gage tabs 
The short lead wires coming from the terminals also had their ends soldered together so that they would not fray. These were short sections that would be twisted to the long wires coming from the DAQ for data collection. These wires can be seen in Fig.( 3.31a). Note that at this point, there is still corrosive flux from the solder that is surrounding the connections. So next, rosin solvent from Micro Measurements was used to remove the flux as well as the tape on the strain gages. After this step the strain gage was protected with liquid called M Coat from Micro Measurements. At this point, the strain gage would be ready for data collection after 20 minutes of letting the M Coat cure. The chord-wise strain gages ready for testing on the wing model can be seen in Fig.( 3.31b).

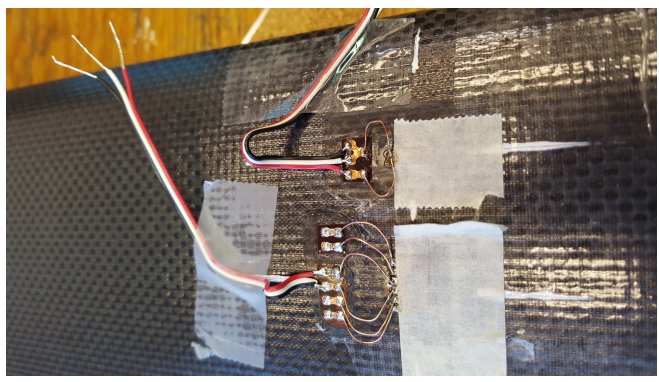

(a) Lead wires soldered into the strain gage and terminals

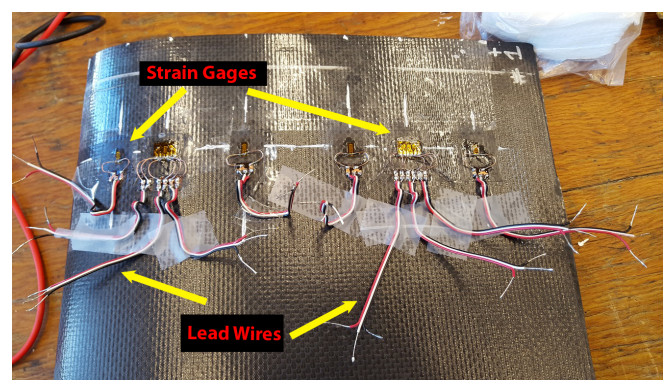

(b) Chord wise strain gages ready for data collection

Figure 3.31: Strain gages bonded and wired onto wing specimen 
Chapter 4

TEST METHODOLOGY

In this chapter different experimental test and supporting equipment are covered. It starts with an explanation of how the DAQ was chosen and its description. It then covers how the DAQ is setup and connected for data collection and recording. The last topic covered the system and software used to collect data.

The next section covers how the material properties of the composite materials were obtained through testing. These included modulus, ultimate strength, and volume fraction properties. Lastly, testing of the cantilever and wing model specimens were covered which included static and fatigue test setups.

4.1 DAQ Selection Process

In order to read the strain values, a measurement device would be needed. The simplest measurement device considered is the P-3500 strain gage indicators by Vishay Micro Measurements. These indicators are frequently used in the aerospace experimental stress analyst course at California Polytechnic San Luis Obispo for static testing. These have been proven to provide reliable data but the reason that they were not used is because the data recording frequency was not adequate. Since the fatigue testing would be done at $10 \mathrm{~Hz}$, it is recommended to have a scan rate of at least 10 times greater than that value. This strain indicator also does not have its own type of data collecting software nor utilize a LabView virtual interface (VI).

The next system considered was a National Instruments (NI) data acquisition 
device (DAQ) with a model number of NI USB - 6229. This DAQ has a sampling rate of $250 \mathrm{kS} / \mathrm{sec}$ for all connected channels. This means that if all 18 strain gages on the wing were connected, the sampling speed for each would be 13,888 $\mathrm{S} /$ sec. This rate would be more than sufficient for the anticipated test frequency. Although, since there is no internal signal amplification, Tacuna Systems strain gage amplifiers model EMBSGB200-M would have to be used. If a signal amplifier were not used, it would make it extremely difficult to register a change in resistance caused by the strain. The signal amplifiers would also need their own power supply adding complexity to the system. This DAQ also did not have its own signal noise suppression or associated software.

A prior student made a LabVIEW VI that would read and record voltages going through the strain gage. When the strain gage is placed in tension or compression the wire filament changes resistance due to its dimensions. This change in resistance would change the voltage passing through the strain gage and be converted to a strain value using its gage factor, excitation voltage and signal. The problem with this system was that the signal noise made it difficult to have a stable reading. This would have resulted in significant error in data collection. There were also only 4 signal amplifiers available for use so only 4 strain gages would be recorded at a time. It would have taken twice as long to gather data from the wing when compared to the DAQ that was ultimately chosen which had 8 channels. The system that was used also required less setup and data collection because of its plug and play aspect.

\subsection{Model 8000 DAQ}

The DAQ ultimately used was a Micro Measurements Model 8000 with 8 channels for data collection and a maximum scan rate of 1000 samples per second for each channel. This system is a plug and play system that also has a dedicated 
software that would calculate the strain measurements from the voltages automatically. It has internal signal filtering done by a Finite Impulse Response multi-stage filter. This filtering allows the signal to be extremely stable by rejecting noise and allowing high measurement accuracy. In the quarter bridge connection used in this thesis there is only $+/-.01 \%$ error and a drift of $2.8 \mathrm{ppm} /{ }^{\circ} \mathrm{C}$ maximum. This allowed exceptional accuracy when collecting data from the strain gages during testing. This system also came with internal signal amplification with gain accuracy of $0.1 \%$ and stability of $+/-30 \mathrm{ppm} /{ }^{\circ} \mathrm{C}$. The excitation voltage used for the strain gages could range from 0 to $+10 \mathrm{VDC}$ with a resolution of $3 \mathrm{mV}$ and accuracy of $+/-10 \mathrm{mV}$. A multi-view representation of the DAQ can be seen in Fig.( 4.1).[17]

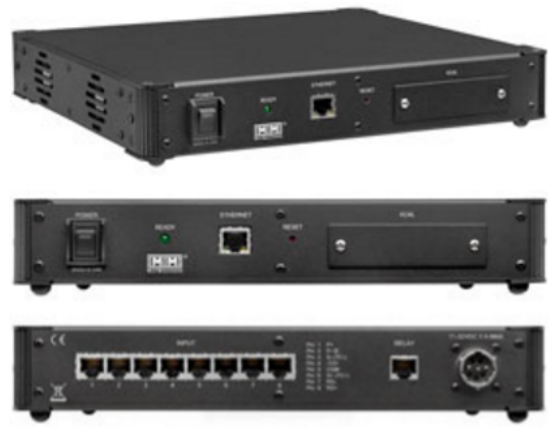

Figure 4.1: Model 8000 DAQ [17]

The DAQ was connected to a laptop with the Strain Smart software installed by the use of a RJ- 45 connector commonly called an Ethernet cable. On the opposite side of the system there were 8 Ethernet ports that could be used for data collection channels. This type of cable consist of 8 individual wires that could be used to connect a single strain gage in a quarter bridge, or for connections of up to 4 strain gages in a full bridge circuit. A close up of the 8 wires in the Ethernet cable can be seen in Fig.( 4.2).[18] 


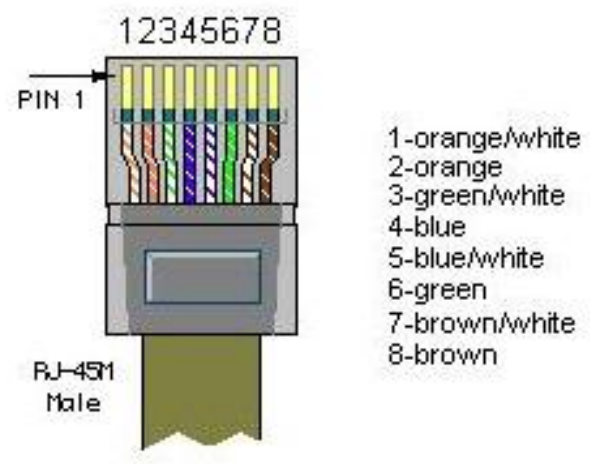

Figure 4.2: Close up of the RJ-45 cable and its 8 wires used for strain gage circuit connections

In order to ease circuit connections, a RJ-45 circuit breakout was used as pictured in Fig.( 4.3). On one side, the Ethernet cable from the machine was connected and on the other was screw locking pins where lead wires could be connected to build the quarter bridge circuits used in this project.

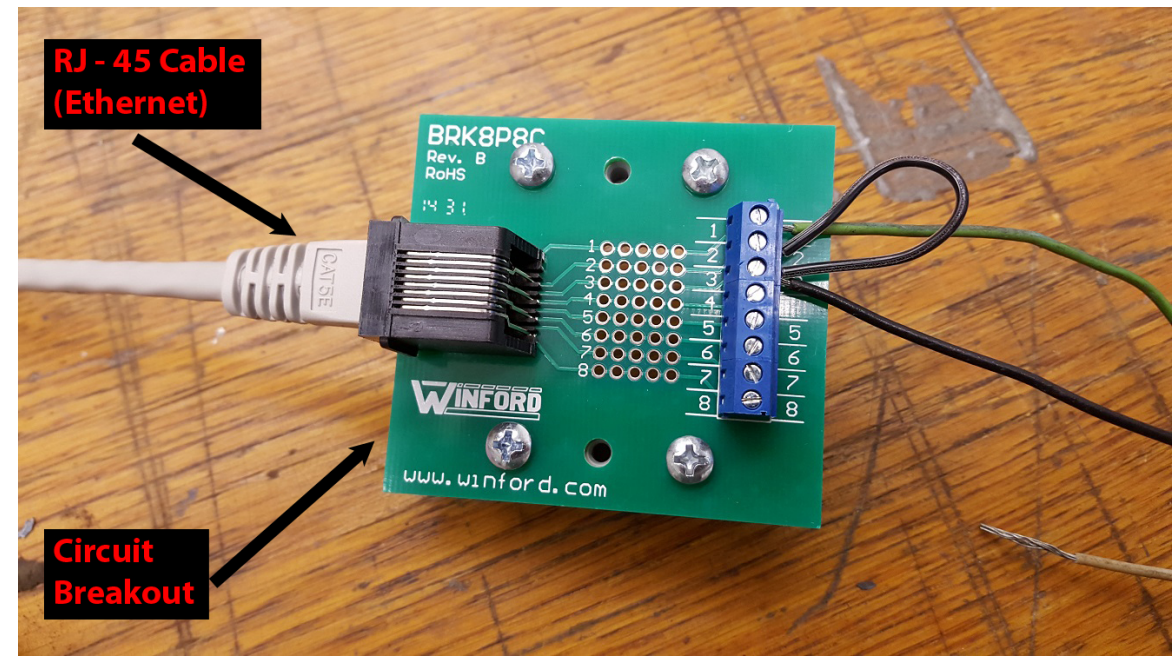

Figure 4.3: RJ- 45 circuit breakout

\subsubsection{Connection and Setup}

The system, connected to the laptop and circuit breakouts, can be seen in Fig.( 4.4). It can be seen that this system is extremely user friendly; only needing one power cable and one Ethernet cable for data transfer to the laptop. For data 
collection, RJ- 45 cables were connected from the DAQ to a circuit breakout which provided a good connection.

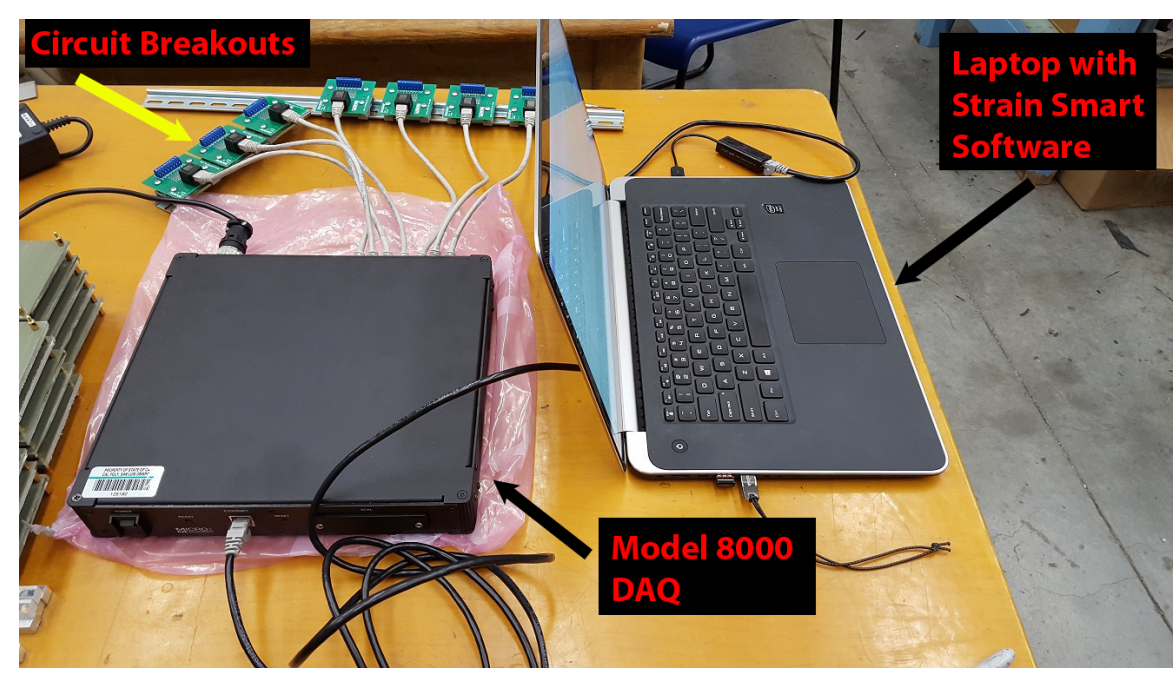

Figure 4.4: DAQ and computer for data collection

Wires were connected to the breakouts that would be connected to strain gages as well as signals from the Instron such as force and displacement. In Fig.( 4.5) the two right breakouts had 3 wires and the two left breakouts had two for the Instron connections. The 2 wire connections were connected to the back of the Instron controller so that force and extensometer readings could be recorded with the DAQ. This allowed for the Instron measurements and the strain gage data to be taken at the same moment in time. 


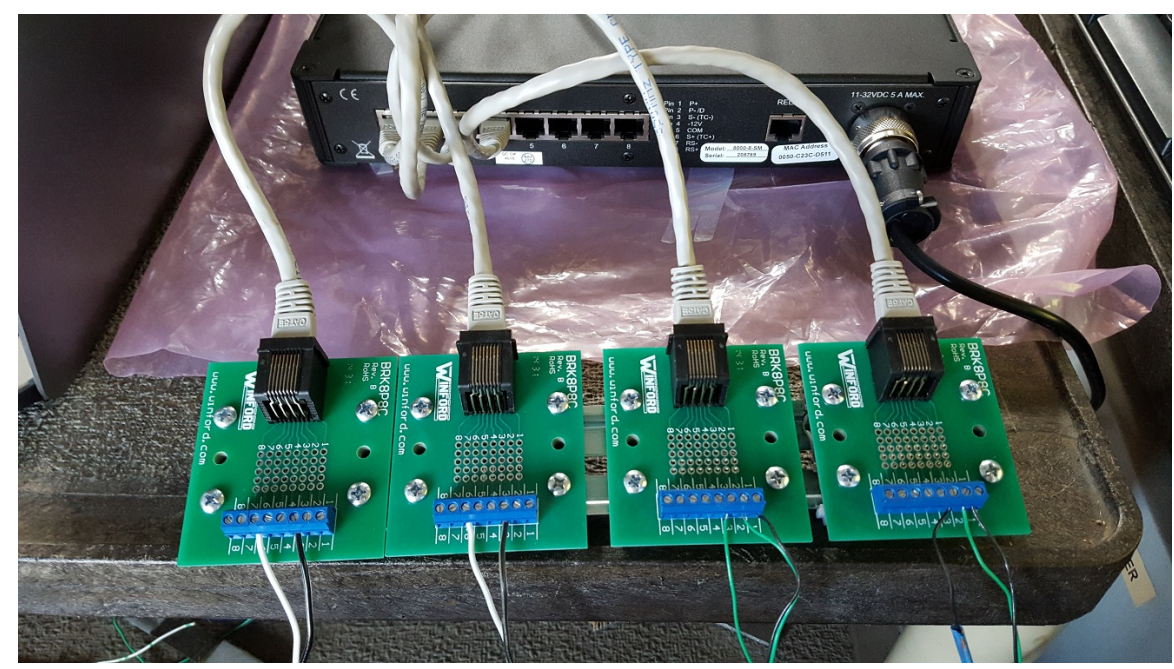

Figure 4.5: Breakouts with 2 wire and 3 wire connections for the instron and strain gage signals respectively

The 3 wire connection was used for the strain gages for its advantages over 2 wire connections. The advantages were compensation for temperature change in the testing environment and canceling out of lead wire resistance. 3 wire setups also allow for a more sensitive measurement allowing small strains to be detected. A demonstration of this was performed by Micro Measurements sales manager, Jim Johnson, where a $20 \mathrm{ft}$ lead wire was connected to a test specimen with a 2 wire connection. The specimen was left unloaded and as he warmed up the wires, the strain changed to a value of around $200 \mu \epsilon$. The same procedure was done to a specimen in a 3 wire connection and the value only deviated from the zero by about $6 \mu \epsilon$. Extreme temperature changes were not expected in my project but the confidence that temperature effects would be negligible was valued. In Fig. (4.6) the connection diagrams can be seen for both the Instron signals which only use 2 wires and the 3 wire connection advantageous for a strain gage.[19] 


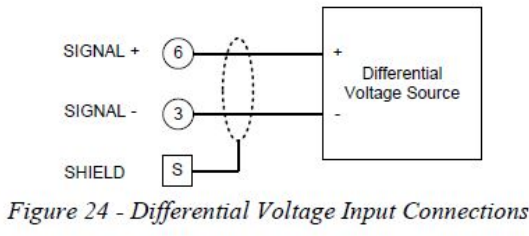

(a) Diagram for Instron signals from the controller

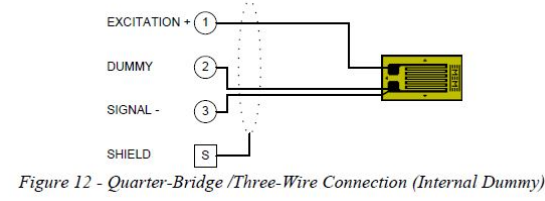

(b) Diagram for signals from strain gages used for data collection

Figure 4.6: Two of the connection types used with Model 8000 DAQ [20]

The strain gages used in this thesis project were manufactured by Vishay Micro Measurements and may be found in their main line of strain gages for engineering purposes. The company provided great support with installation, operation instructions and recommendations. The two strain gages types used were uni-axial and rosettes with nominal resistances of 350 and 120 ohms respectively. The uniaxial strain gage had a gage factor of 2.16 where the rosette had a gage factor of 2.08 for the outside strain gages and 2.1 for the center strain gage. Fig.( 4.7) shows a close up of the uni-axial strain gage used on various structures throughout this study. The $350 \mathrm{ohm}$ resistance strain gages are more sensitive to strain changes making them more accurate while the $120 \mathrm{ohm}$ are cheaper to manufacture. The $120 \mathrm{ohm}$ strain gages still offer exceptional accuracy and were used confidently in this project for data collection. 


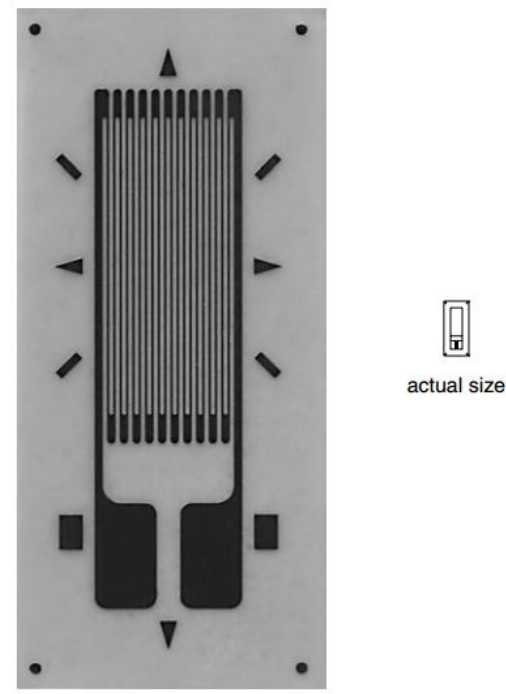

Figure 4.7: Close up of uni directional strain gage used in experiments

\subsubsection{Strain Smart Software}

The strain smart software that was designed to be used with the series 8000 DAQ is a windows based software used for collecting data from strain gages as well as presenting and storing it. It is made by the same company as the DAQ used therefore they work together seamlessly. It is a ready to use software that makes it easy to conduct a test and collect data from strain gages. The results from the test are automatically converted into engineering units so that the operator can analyze a test on the spot. Minimal post processing of data is required saving time for the operator.

If thermal affects would have been an issue in testing, the software would have the capability to adjust strain results to negate the thermal effects. This would be done by connecting a thermal couple to the DAQ and inputting the strain gage's strain and temperature relationships given by the manufacture. The DAQ would then adjust the strain value based on its temperature.

The software allowed for easy calibration of strain gages with a touch of a button and then zeroed just as easily. It also allowed for shunt calibration of 
strain gages which is used to scale the resistance reading of the active strain gage so that all the resistors in the Wheatstone bridge are balanced. This is done by replacing the active strain gage labeled as $R_{1}$ in Fig.( 4.8$)$ with a precise resistor of known value labeled as $R_{C}$. After forcing the active strain gage to be a known resistance value the rest of the completion resistors in the circuit can be measured and their deviation can be used to scale the active strain gage resistance. [21]

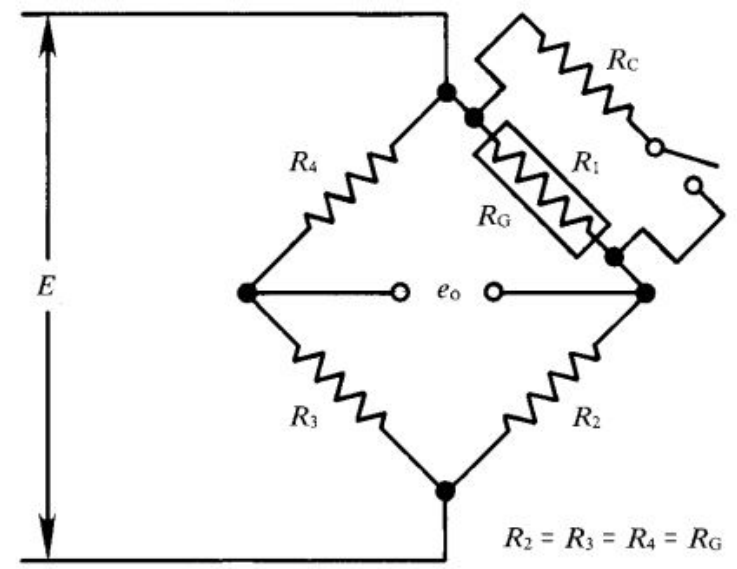

Figure 4.8: Wheatstone bridge circuit showing the active strain gage and the shunt calibration resistor

The software also allows for multiple channels of results to be displayed in real time in different ways. In Fig. (4.9) results for a test can be seen as a bar graph or as a time dependent signal. The bar graph plots were useful during static testing where it could be seen if the data was as expected and then recorded. To record, the user could decide between continuous data capture or by pressing a record button, one sample could be recorded at instant. For fatigue testing it was also possible to set time increments between data capture and select how many samples would be recorded at that time. This allowed the data files to be far smaller than continuously taking data throughout the 3 hour fatigue test. 


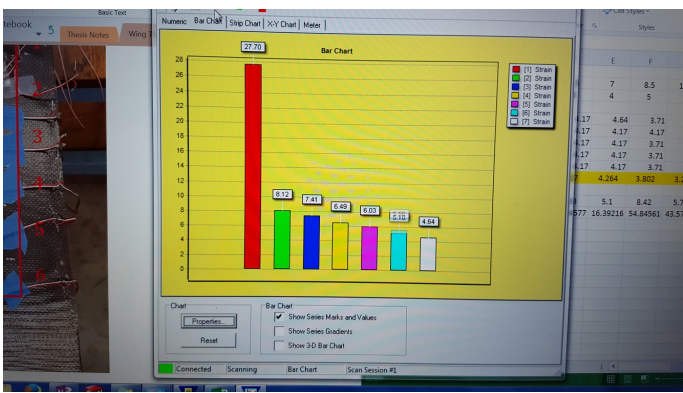

(a) Bar graph representation of strain data from test

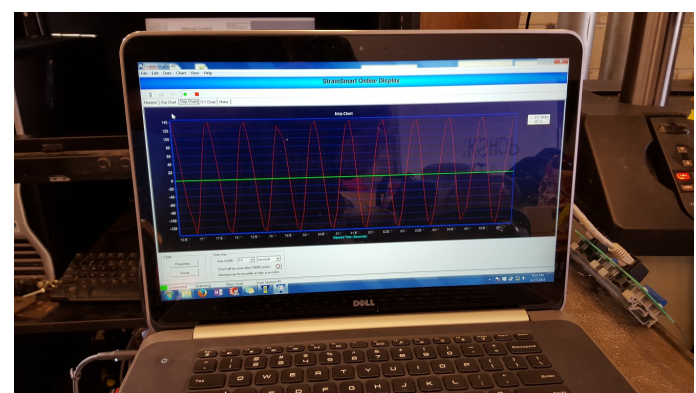

(b) Time dependent plot of strain data from test

Figure 4.9: Two types of ways that Strain Smart software can display results

Once the data was recorded it would be saved to the software's database where it could then be exported as a text file or even an Excel spreadsheet. For this project the data was exported as an Excel file so that the program's mathematical functions could be used to compile data and make plots.

\subsection{Material Properties}

In this section the procedures of how the strengths, modulus, and volume fraction of the composite materials were determined is discussed. The first subsection covers how the uni-axial tests were setup and conducted. Dimensions of the specimen along with measurement devices are covered. In the next subsection the procedures for determining the volume fraction of the composite materials were covered.

\subsubsection{Uni-axial Test Setup}

The carbon fiber used in this thesis was donated from aerospace companies to be used for Cal Poly projects. Since donated material tends to be expired or damaged in some way, the material properties from the manufacture can not be trusted. In order for the FEA model to correctly represent the experimental testing the properties were tested for using a model 8801 servo-hydraulic Instron 
machine. The testing followed procedures listed in ASTM D3039.[22] Poisson's ratio was also tested for according to the procedures in ASTM E132 - 04.[23] Since the materials used were weaves it was assumed that the material behaves the same in the 0 and 90 degree orientations. [24]

First a 12 " by 12 " flat plate was made in the Tetrahedron heat press according to the material cure cycle. The manufacture specifies a specific temperature and pressure that needs to be applied to the material for it to cure correctly.[25, 26] Once this is done, the test specimens are cut to the dimensions called for in their respective ASTM procedures. For the tensile samples the dimensions were 10 inches long by 1 inch wide and about .1 inch thick. A drawing of the tensile sample with dimensions can be seen in Fig.(4.10). Two inch metal tabs that were used for gripping can also be seen in the figure below.

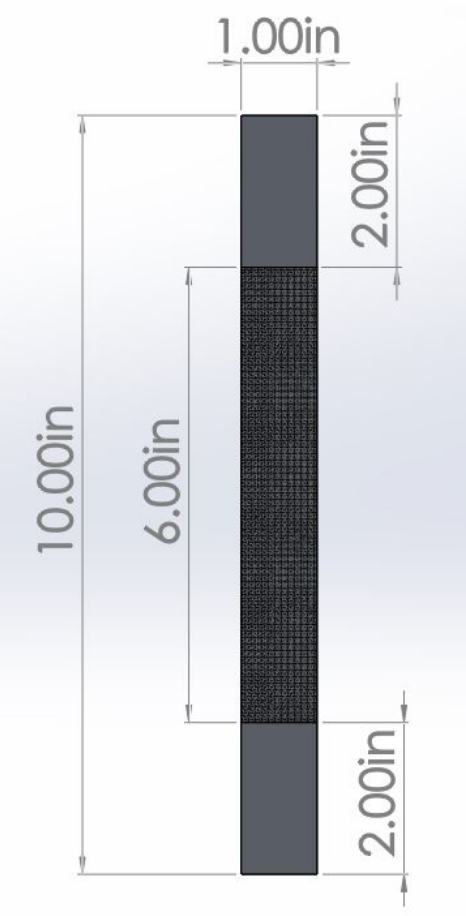

Figure 4.10: Drawing of tensile test specimen

Since these samples were expected to be tested at a load higher than 6000 lbf, emery cloth would not be suitable, for gripping. Therefore metal tabs would 
need to be bonded onto the ends of the specimens for the Instron to grip onto. In order to provide a good bond the surface of the specimen was abraded and then cleaned using isopropyl alcohol. Next the heat press was heated to $150^{\circ} \mathrm{F}$ while the Loctite EA 956 adhesive was mixed according to the data sheet and applied to the specimens.[27] Tensile specimens and the aluminum tabs can be seen in Fig. (4.11) below.

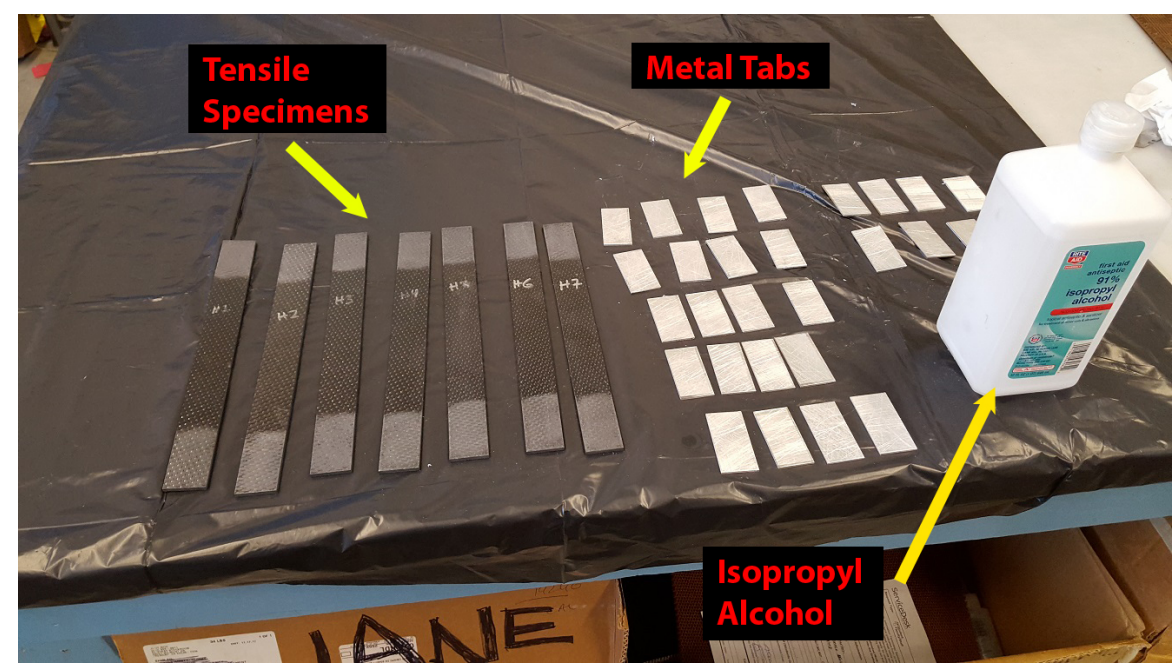

Figure 4.11: Abraded Tensile Specimens and Metal Tabs

The specimens are then put between two steel plates and into the press for a few minutes to let the adhesive gel. Then it was then taken out and the tabs were aligned and cured for an additional 2 minutes. The tensile samples can be seen in Fig.( 4.12) on top of the steel plate. 


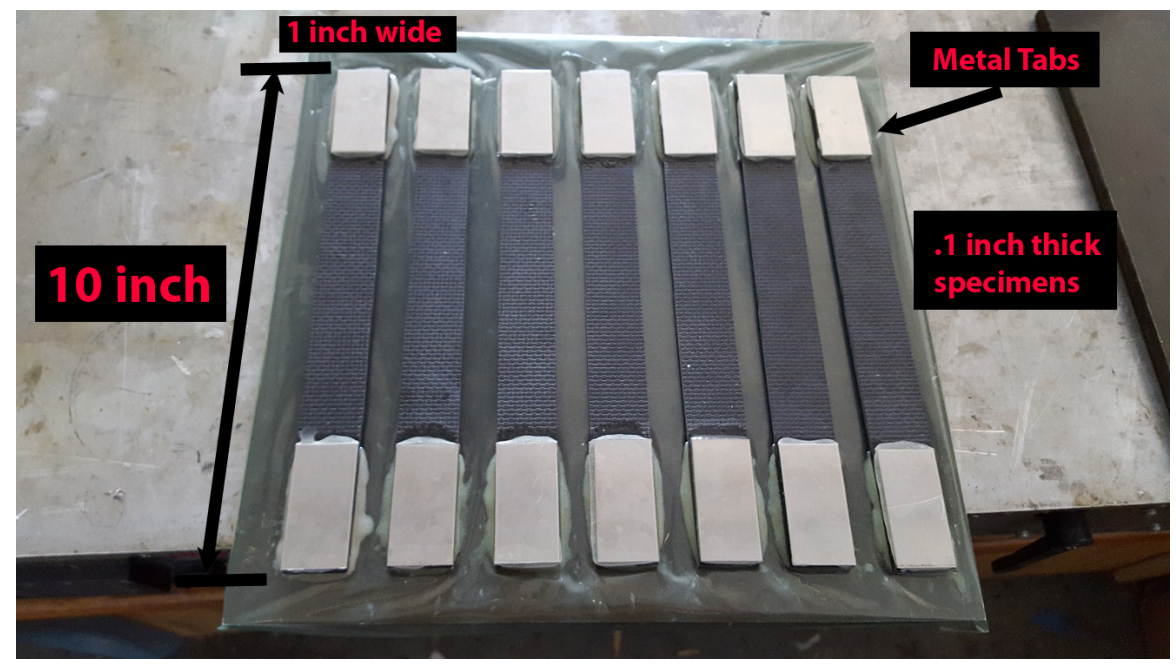

Figure 4.12: Tensile specimens

After the samples were finished, strain gages were bonded in the axial and transverse directions so that the Poisson's ratio of the material could be calculated. The strain gages on a test specimen can be seen in Fig.( 4.13) along with wires that are connected to the DAQ.

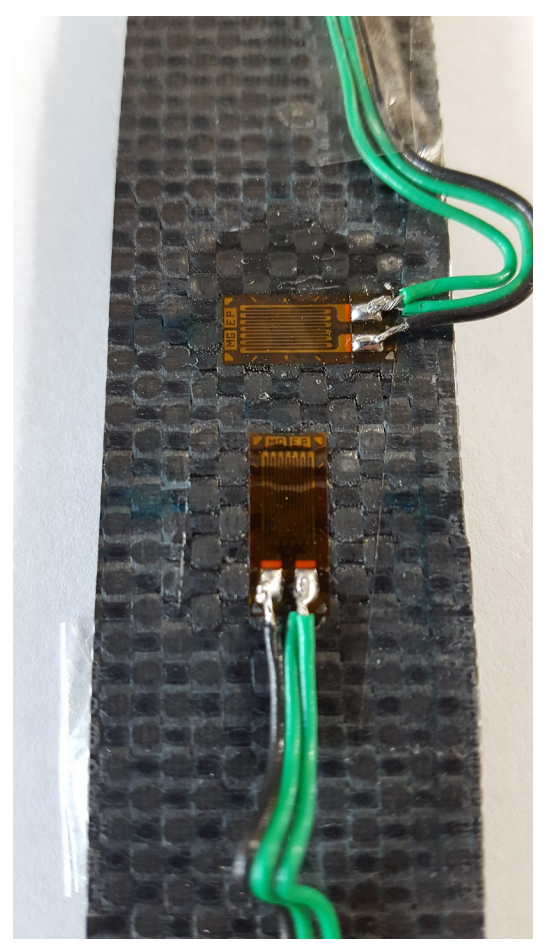

Figure 4.13: Specimen with strain gage 
Following the ASTM procedures the Instron actuator was set to displace at 0.05 inches per minute while the load, displacement, were being recorded. Since the specimen dimensions were programmed into the Instron software it automatically calculated stress and strain.

An extensometer was used for collecting displacement data since it can accurately determine displacements in the test specimen. A tensile specimen made out of AL 6061 was tested and it was found that the modulus was off by about $50 \%$ using the Instron displacement. After attaching an extensometer and strain gages onto the same specimen the modulus matched the expected modulus value from the manufacture very well. Therefore the carbon fiber specimens all would be tested with an extensometer and a few specimens will have strain gages for assurance. The ones with strain gages will also have them placed transversely to calculate Poisson's.

An extensometer was used to verify that the strain gages were reading correctly during testing. The extensometer works by holding one of its knife edges fixed onto to the specimen while the other is free to displace with the material when under tension. The extensometer on the test specimen can be seen in Fig.( 4.14). Once the displacement was accurately read the strain was calculated using its gage length of $0.5 \mathrm{in}$. The strain data from the strain gages and the extensometer displacement were recorded using the Model 8000 DAQ and its Strain Smart software. 


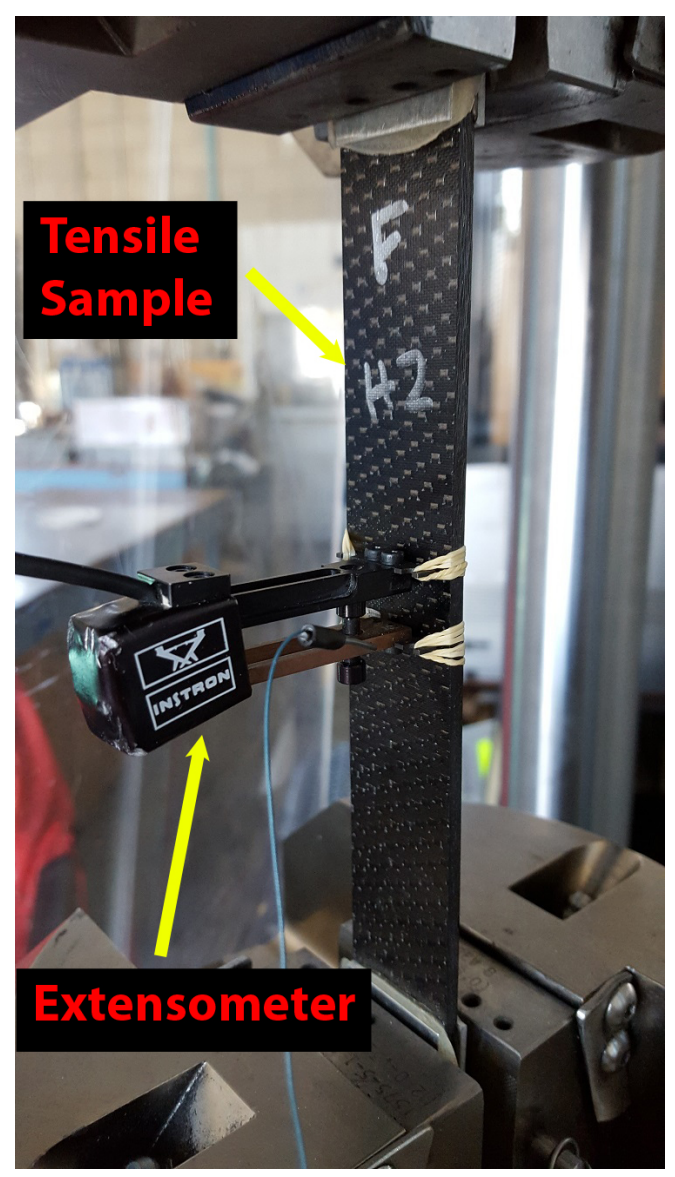

Figure 4.14: Instron extensometer on tensile specimen

Since the extensometer would be damaged if the sample would fail, an initial sample without the extensometer was tested to find the ultimate strength. The failure criterion that was used was a 40 percent load drop per the ASTM. Then the samples with and without strain gages along with the extensometer would be loaded to $34 \%$ of the ultimate load. Data collected showed a linear relationship that would be used for the modulus calculations. A tensile specimen along with a strain gage can be seen in the Instron grips in Fig.( $4.15 \mathrm{a})$. Once data for the modulus calculation was collected, the specimens without strain gages were failed to find their failure load. A desired failure is shown in Fig.( 4.15b) where it occurs near the center of the gauge length. 


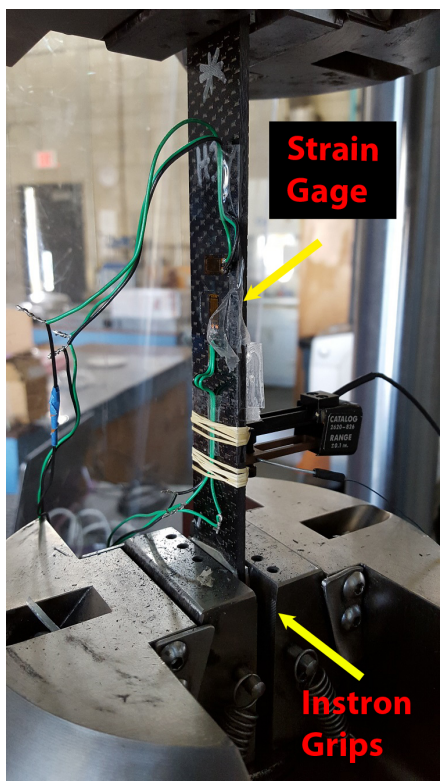

(a) Specimen in Instron grips

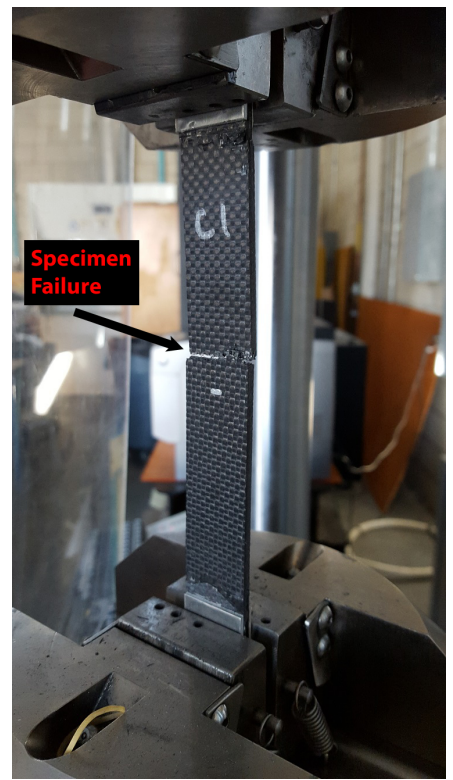

(b) Failed tensile specimen in Instron grips

Figure 4.15: Test specimens in Instron grips

After all of the specimens were tested, the data was collected and post processed for calculations. The stress and strain of each test specimen was calculated since they would be used to find the modulus of the material. The equations that were used for these calculations can be seen in eq.( 4.1) and eq.( 4.2) respectively.

$$
\begin{gathered}
\sigma=\frac{F}{A} \\
\epsilon=\frac{\Delta L}{L}
\end{gathered}
$$

where $\sigma$ is the axial stress in (psi), $\mathrm{F}$ is the axial force in lbf, $\mathrm{A}$ is the crosssectional area in $\left(i n^{2}\right), \epsilon$ is the axial strain in (in/in), $\Delta \mathrm{L}$ the change in length of the specimen in (in), and $\mathrm{L}$ is the gage length of the specimen in (in).

In order to calculate Poisson's ratio both the axial and transverse strains from the strain gages were plotted against the axial stress. After this was done the slope of the axial strain vs. axial stress was divided by the slope of the transverse 
strain vs. axial stress. This calculation can be seen in eq.( 4.3) below and note that there is a negative since the transverse strain is in compression. The Poisson's ratio conceptually is how much the material deforms perpendicularly to the force compared to the load direction.

$$
\nu=\frac{\frac{\partial \epsilon_{a}}{\partial \sigma}}{-\frac{\partial \epsilon_{t}}{\partial \sigma}}
$$

where $\nu$ is the Poisson's ratio and the subscripts ${ }_{a}$ and $t_{t}$ are axial and transverse designations.

\subsubsection{Fiber Volume Fraction}

In order to investigate how well the laminates cured, a volume fraction study was done. The data sheet specifies a nominal ratio between the fiber and matrix based on each of their volumes. Although this ratio is for an ideal cure cycle and in reality the ratio can vary from this value. In order to determine the actual volume fraction of the laminate, the epoxy is burned off allowing just the weight of the fiber to be left. This method is outlined in ASTM D2584 with exact procedures.[28] One of the test specimens used in this test is pictured in Fig. (4.16). Most woven composites have fiber volume ratios that are in the $35 \%$ to $55 \%$ range which was found to be the optimal for strength.[29] 


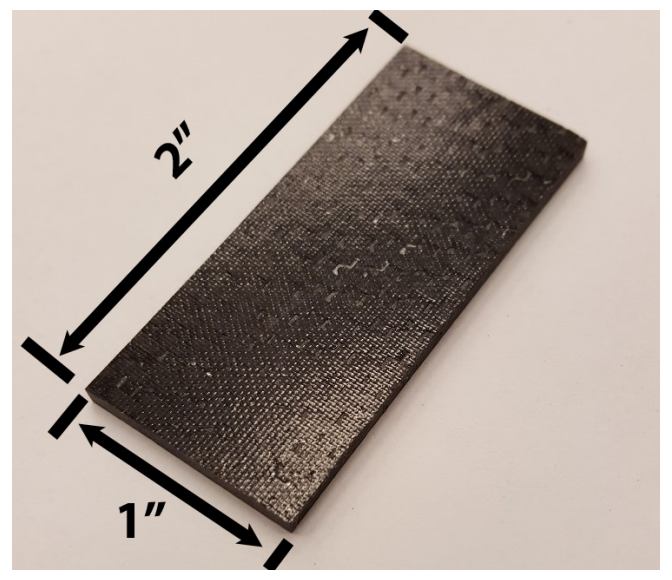

Figure 4.16: Sample used in volume fraction testing per ASTM D2584

First specimens were cut into a rectangular shape from the composite laminate plate. The weight of each specimen should weigh about $5 \mathrm{~g}$ and was measured for volume calculations. After this an oven was pre-heated to a temperature of $1050^{\circ} \mathrm{F}$ for ten minutes. Then a metal plate was pre-heated to clean off any contaminates. It was then weighed by itself and with one of the test specimens. Next both the plate and the specimen were placed in the oven until the epoxy burned completely away. The oven can be seen in Fig.( 4.17) with one volume fraction sample inside on a metal plate. 


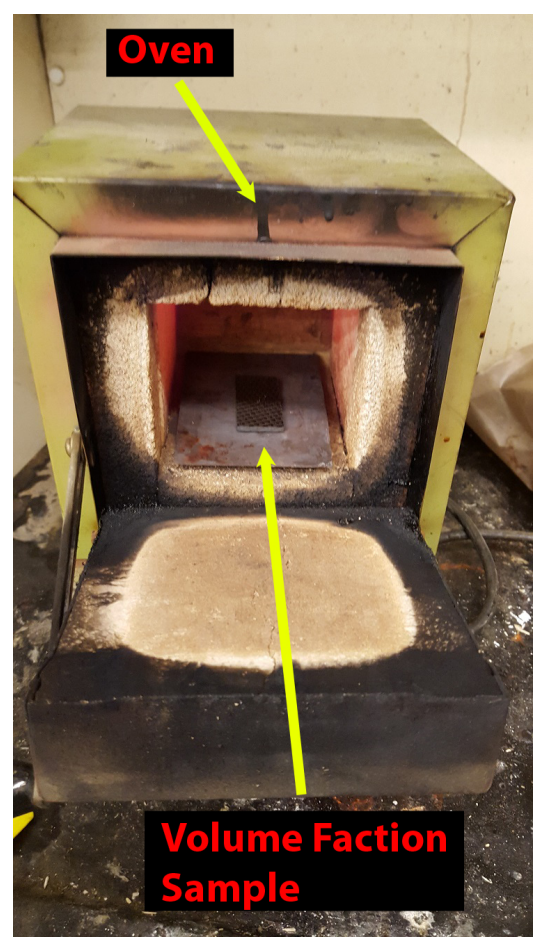

Figure 4.17: Oven used to burn the epoxy out of the carbon sample for volume faction calculations

The sample was ready to be taken out when the fibers turned shiny and the layers of the fabric could be seen without the epoxy. For both materials this took about 40 minutes to an hour. The plate was taken out of the oven to cool off and weighed along with the fibers. The necessary measurements had now been taken so that the fiber volume fraction could be calculated. In Fig.( 4.18) a cooked sample can be seen where the fibers appear shiny since the epoxy had been burned off. It also shows the layers of bare fibers picked up so that individual fibers can be seen. 


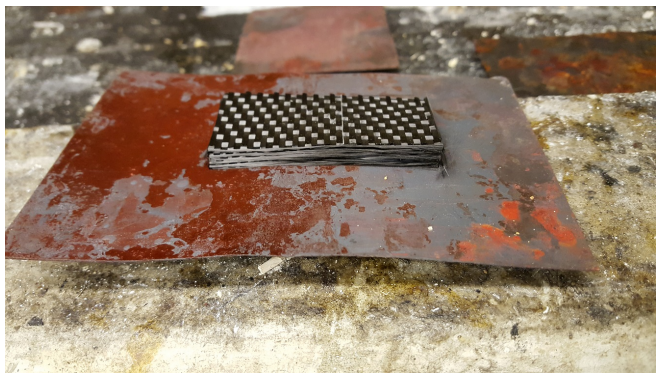

(a) Volume fraction sample after being pulled out of oven

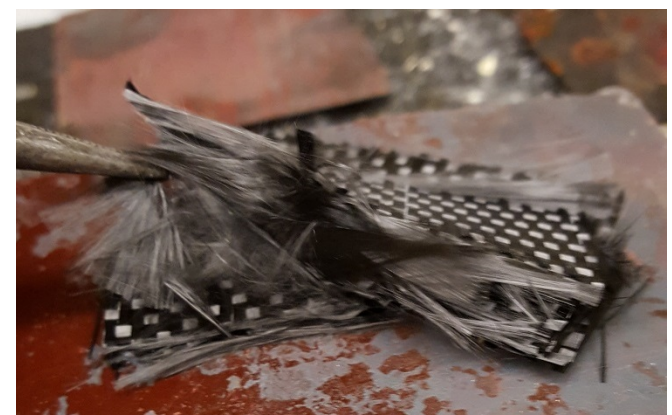

(b) Individual fibers can be seen in the specimen after burning epoxy

Figure 4.18: Example of specimen after being cooked in the oven

The equation used for each specimen of the two composite materials is shown in eq. (4.6)

$$
\begin{gathered}
K_{1}=\frac{w_{\text {lam }}}{\rho_{\text {lam }}} \\
K_{2}=\frac{w_{\text {matrix }}}{\rho_{\text {matrix }}} \\
V_{f}=\frac{K_{1}-K_{2}}{K_{1}}
\end{gathered}
$$

where $K$ is a substituted variable, $V_{f}$ is the fiber volume fraction ratio, $w$ is the weight in lbs, $\rho$ is the density in $\frac{l b}{i n^{3}}$, subscript lam denotes the laminate and subscript matrix denotes the epoxy.

For the Cycom material the laminate density was not given, therefore it was replaced with the measured density of the specimen. This was calculated using the eq. (4.7)

$$
\rho=\frac{w}{V}
$$

where $V$ is the measured volume of the specimen in $i n^{3}$. 


\subsection{Validation for Experimental Static Test}

After the material property testing was complete, different types of static test were conducted with applied loads. The first one covered in this section was a cantilever beam test of a composite laminate. The purpose of this was compare it to an FEA model to insure that experimental material properties are correct. After this was done physical loads were put on the wing specimens while taking data from the strain gages on the surface. Since during the Instron fatigue testing the load cell was not connected to the wing, a force and displacement study was done to estimate the force being applied.

\subsubsection{FEA Model Validation}

The first cantilever beam study that was done was on a 26 in long aluminum beam used in the aerospace experimental stress analysis course. The cross section of the beam measured 1.506 inches wide by 0.255 inches thick. The beam already had two strain gages positioned at 4 and 8 inches away from the fixed end. A load of 1.316 pounds was added one inch away from the end of the beam and the strains were collected simultaneously. The cantilever beam and its constraint can be seen in Fig.( 4.19) below. The strains collected from the test would then be compared to the FEA results. If the results are in agreement, then this would give confidence in how the FEA model is being constrained and loaded. Since the wing would be loaded in a similar fashion we hope to prove that the simulation works for a simple case initially. 


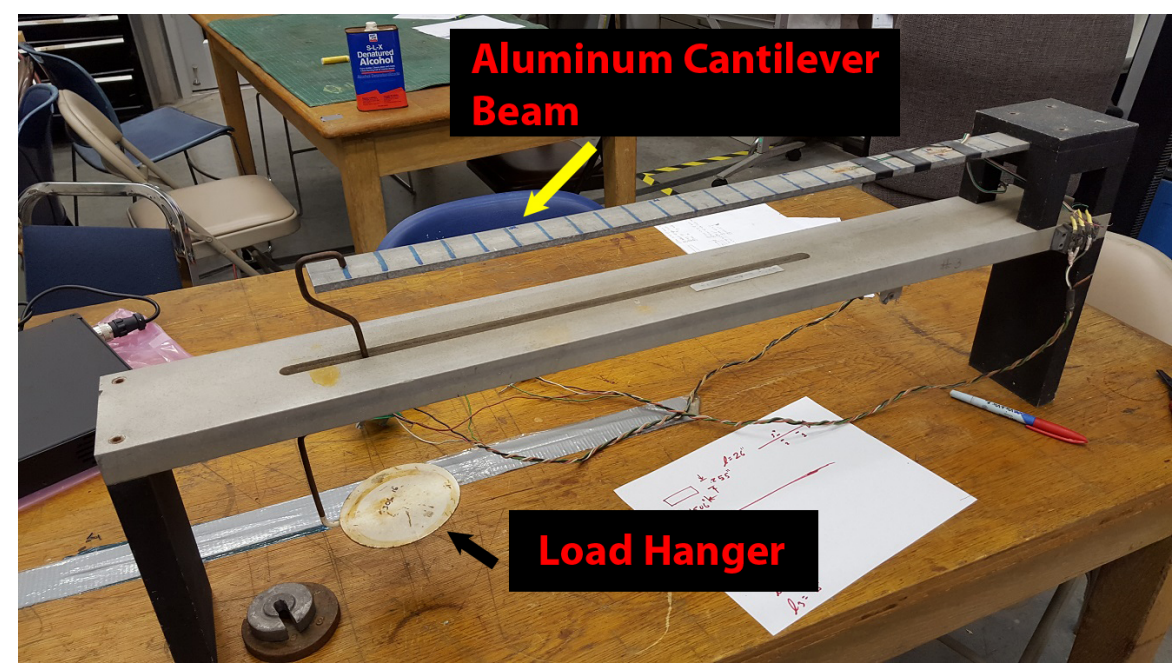

Figure 4.19: Aluminum cantilever beam experimental test setup

To compare to the experimental and numerical methods an analytical solution for the maximum displacement and the strain at the strain gage locations was done. In order to calculate the strain at strain gage locations the moment equation was derived for the beam. The equation is shown below in eq.( 4.8$)$

$$
M=-M_{1}+P x
$$

where $M$ is moment in terms of length from the fixed end in (in-lbf), $M_{1}$ is the reaction moment from the load in (in-lbf), $P$ is the load in (lbf), $x$ is the distance from the fixed end in (in). In Fig.( 4.20) the variables used in the formula can be seen in the free body diagram of the beam. 


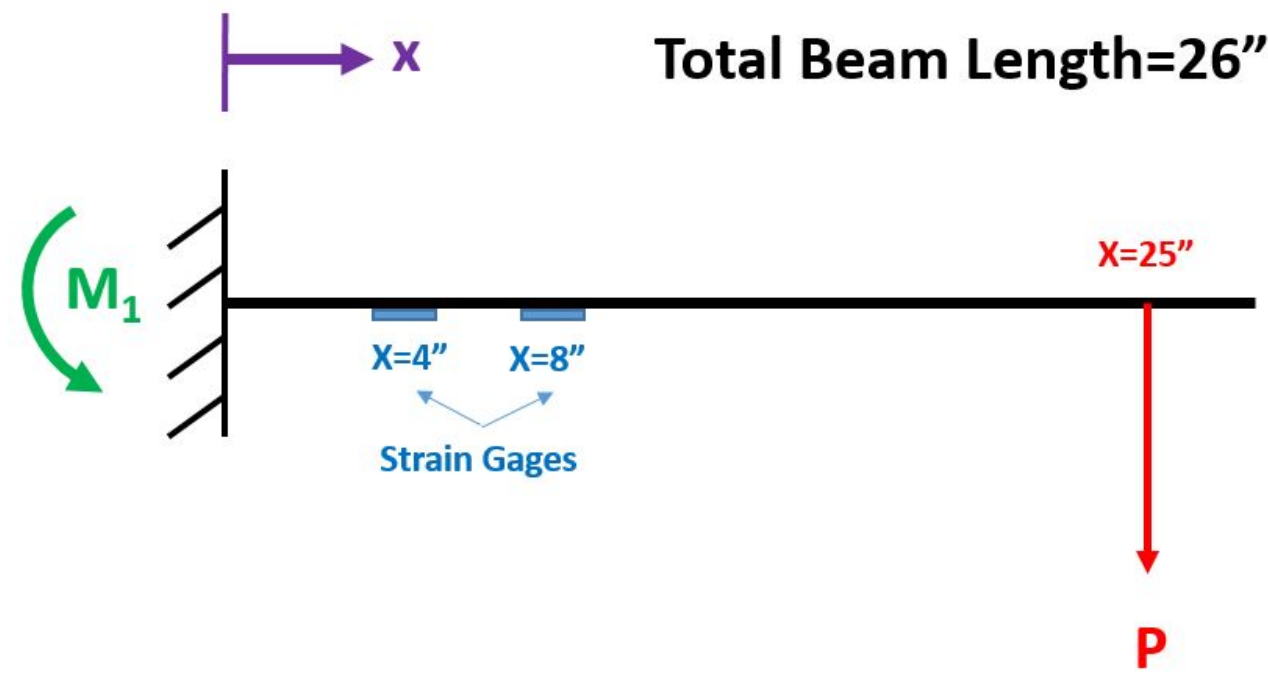

Figure 4.20: Free body diagram of aluminum beam

Once the moment for each location was found it was then used in eq.( 4.9) to calculate the bending stress.

$$
\sigma=\frac{M c}{I}
$$

where $c$ is the distance from the neutral axis in (in), and $I$ is the second moment of inertia of the beam in $\left(i n^{4}\right)$.

Once the stress was found, Hook's law could be used as shown in eq. (4.10) . The reason this equation was valid is because the beam is homogeneous, isotropic, and loaded in the linear elastic region

$$
\sigma=E \epsilon
$$

where $E$ is the theoretical tensile modulus in (psi). Since the modulus for the material was known from the data sheet the strain could then be calculated for each location.

Then the theoretical maximum displacement was calculated using an equation 
found in an online source, for a loaded beam at any distance from the fixed end. Since the beam was loaded one inch away from the tip this equation made it trivial to use since it was in terms of this location. The equation can be seen in the screen shot in Fig.( 4.21).[30]

\begin{tabular}{|c|c|c|c|}
\hline 2. Cartilever Be & ncentrated lo & & \\
\hline 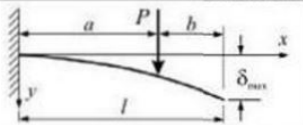 & $\theta=\frac{P a^{2}}{2 B I}$ & $\begin{array}{l}y=\frac{P x^{2}}{6 E I}(3 a-x) \text { for } 0<x<a \\
y=\frac{P a^{2}}{6 E I}(3 x-a) \text { for } a<x<l\end{array}$ & $\delta_{\operatorname{mat}}=\frac{P a^{2}}{6 E I}(3 l-a)$ \\
\hline
\end{tabular}

Figure 4.21: Equation used in analytical calculation of maximum displacement

where $\delta_{\max }$ is the max displacement in (in), $a$ is the load location from the fixed end in (in), and $l$ is the total length of the beam in (in). Since the displacement of the beam was not measured during the experimental test this result will only be compared to the FEA simulation.

In order to assist in validating the FEA model, a simple cantilever test of each composite material was conducted. If the numerical and experimental results are similar then it would give confidence to the material testing as well as the FEA composite material modeling. An analytical solution was also done to compare to the other two analysis methods. A specimen with dimensions of 11 in $\mathrm{x} 1$ in and .1 in thick was cut from the same plate as the tensile samples. Using a metal tab and a clamp, one side was held fixed while the other side would be loaded with the use of a metal hanger and weights. The test setup including the beam and constraints can be seen in Fig.( 4.23). Then strain gages were bonded along the length of the beam at the following distances from the edge of the clamped area: $0.5,1.5,3.0,4.5,6.0,7.5,9.0$ inches. A close up of two strain gages can be seen in Fig.( 4.22) with lead wires solder on to its tabs. 


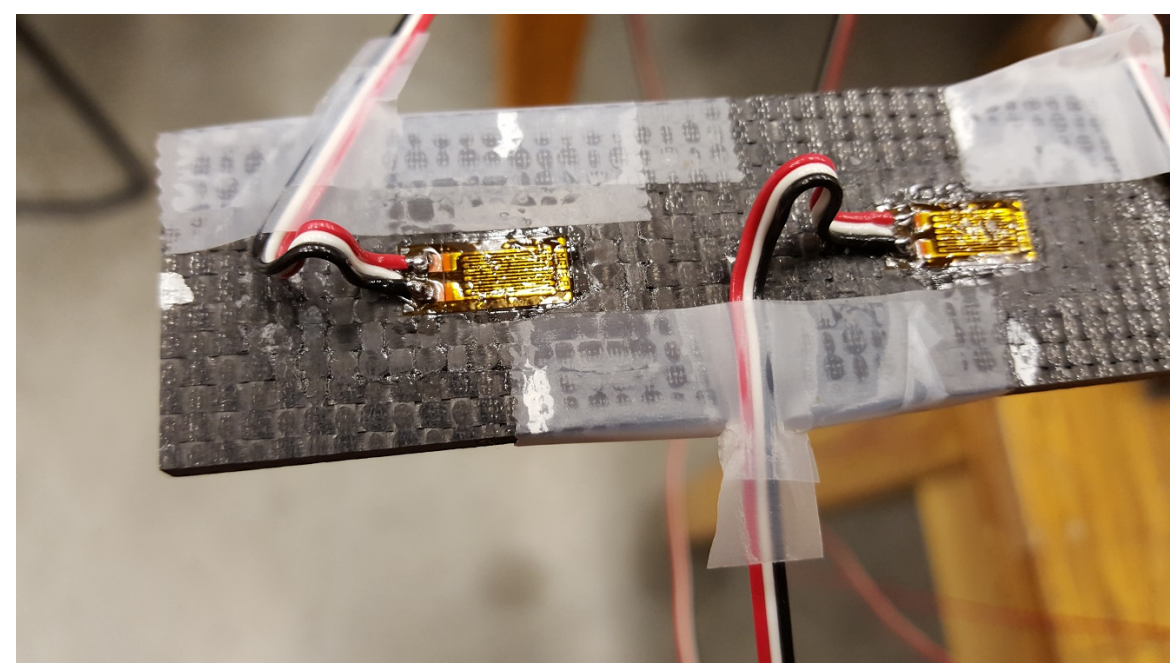

Figure 4.22: Close up of strain gages bonded onto cantilever beam specimen with wires

The strain gages were all connected to the DAQ and data was taken simultaneously. The setup instructions of the DAQ and computer used to take data are in Appendix A and B of this paper. Once the beam was constrained, the strain gages were zeroed and shunt calibrated, and data collection was started. A total force of $1.302 \mathrm{lbf}$ was added to the end which included the hanger and weights. The software allows for real time plotting of all the connected strain gages. It made it easy to make sure that the strain gages are reading correctly since the strains were highest at the constraint and least near the tip. After the readings showed a stable value the load was removed and the data collection was stopped. The test was repeated 5 times on the same specimen to make sure that the tests were consistent. This procedure was done for the two materials used in the thesis with strain gages in the same locations. 


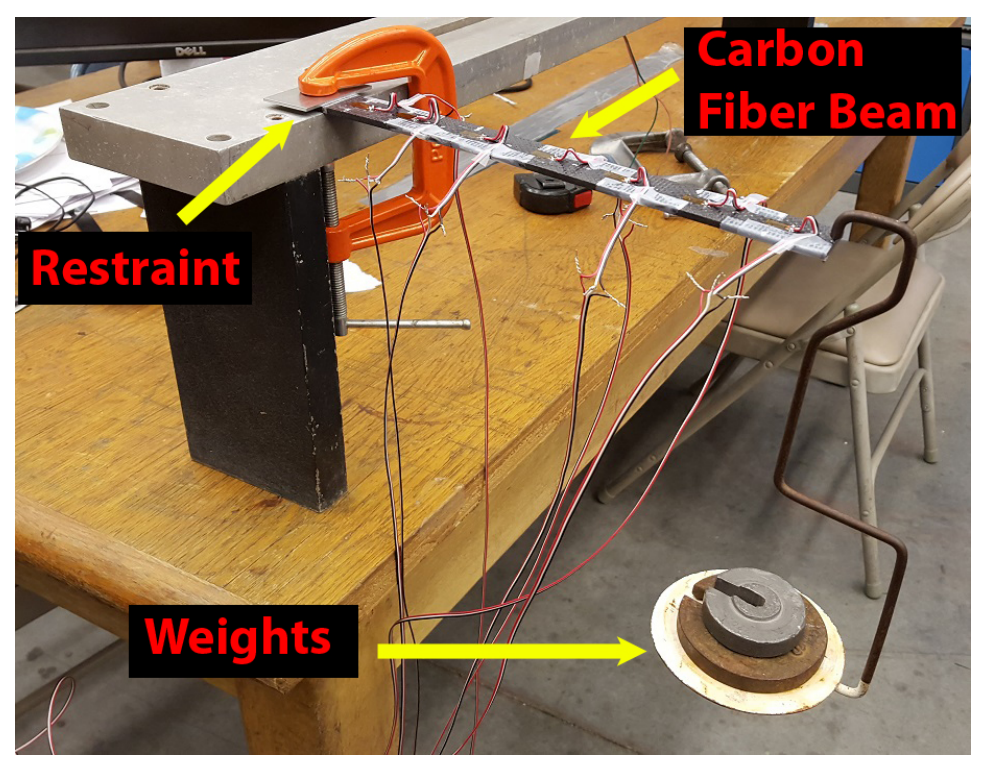

Figure 4.23: Carbon fiber cantilever testing

After the test was conducted, the results would also be compared to an analytical calculation. Since the material used in the beams was carbon fiber the equations used would need to model othrotropic materials. It can be seen in eq. (4.11) that the strain along the beam can be calculated by filling out the compliance and stress matrices for the cantilever beam. The first assumption used in this analysis, that only 2 dimensions are taken into account, therefore any out of plane forces are ignored.[31]

$$
\{\epsilon\}=\{S\}\{\sigma\}
$$

The matrices which show the variables that make up the vectors in the previous equation can be seen in Fig.( 4.24) . The direction 1 is defined along the long axis of the beam where the direction 2 is in the vertical direction perpendicular to the length of the beam. The free body diagram showing the beam boundary conditions, loads, and the direction orientations can be seen in Fig.( 4.25) . 


$$
\begin{gathered}
\{S\}=\left(\begin{array}{ccc}
S_{11} & S_{12} & 0 \\
S_{12} & S_{22} & 0 \\
0 & 0 & S_{66}
\end{array}\right) \\
\{\sigma\}=\left(\begin{array}{c}
\sigma_{1} \\
\sigma_{2} \\
\tau_{12}
\end{array}\right) \\
\{\epsilon\}=\left(\begin{array}{c}
\epsilon_{1} \\
\epsilon_{2} \\
\gamma_{12}
\end{array}\right)
\end{gathered}
$$

Figure 4.24: Matrix equations used in the analytical calculation

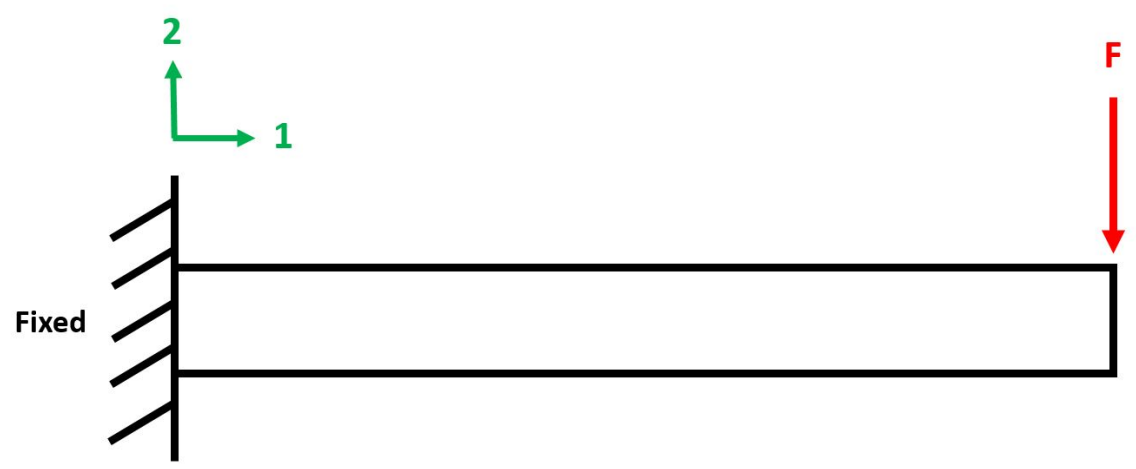

Figure 4.25: Free body diagram of cantilever beam problem

Since the cantilever beam was loaded in pure bending there is only a stress magnitude in the 1 direction. Therefore, by matrix math the resulting equation can be used to solve the problem, eq.( 4.12).

$$
\epsilon_{1}=S_{11} \sigma_{1}
$$

where $S_{11}$ is defined in eq.( 4.13$)$ which reduces the equation to essentially the same as was used for an isotropic cantilever beam problem.

$$
S_{11}=\frac{1}{E_{1}}
$$


The stress in the axial direction from the bending load can be seen in eq.( 4.9) which is the same as the one used in the aluminum beam calculation. The modulus used in the equation is the one from the data sheet for the respective material. Since the carbon materials were woven fabrics the modulus was the same in the 1 and 2 directions.

$$
\epsilon_{1}=\frac{\sigma_{1}}{E_{1}}
$$

In order to find the strain along the beam, the corresponding moment would be used since it was different along the beam. By doing this the strain distribution along the beams could be solved analytically. Once all the calculations were made for the two materials, their results were compared to the other two analysis methods.

\subsection{Experimental Static Test of the Wing}

During the static testing of the wing it will be loaded at one end while fixed on the opposite one. Under load, strains on the top surface of wing will be measured using strain gages. These strain measurements will be used to determine whether the FEA model would be able to correctly model the behavior of the wing. The test schematic that will be explained in this section is shown in Fig.( 4.26). 


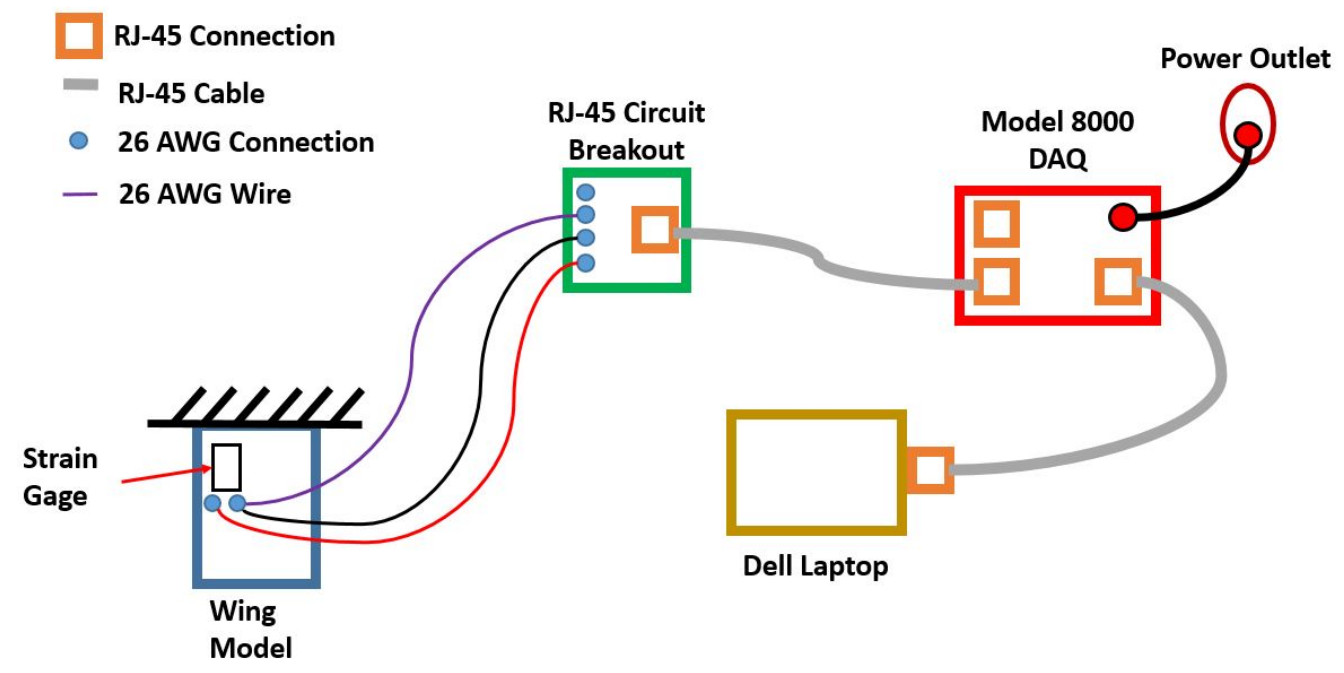

Figure 4.26: Experimental test schematic

After the strain gages on the wing were bonded and wired the wing model was attached to the wing fixture. Then the fixture assembly was bolted to the rigid cast iron angle plate. The wing model constrained to the metal fixture along with the angle plate are shown in Fig.( 4.27). The angle plate is substantially heavier than the wing and its loads but it was clamped down to a fixed table for assurance. Since only one edge of the angle plate was clamped, 30 pounds of weights were added on top of the angle plate as well.

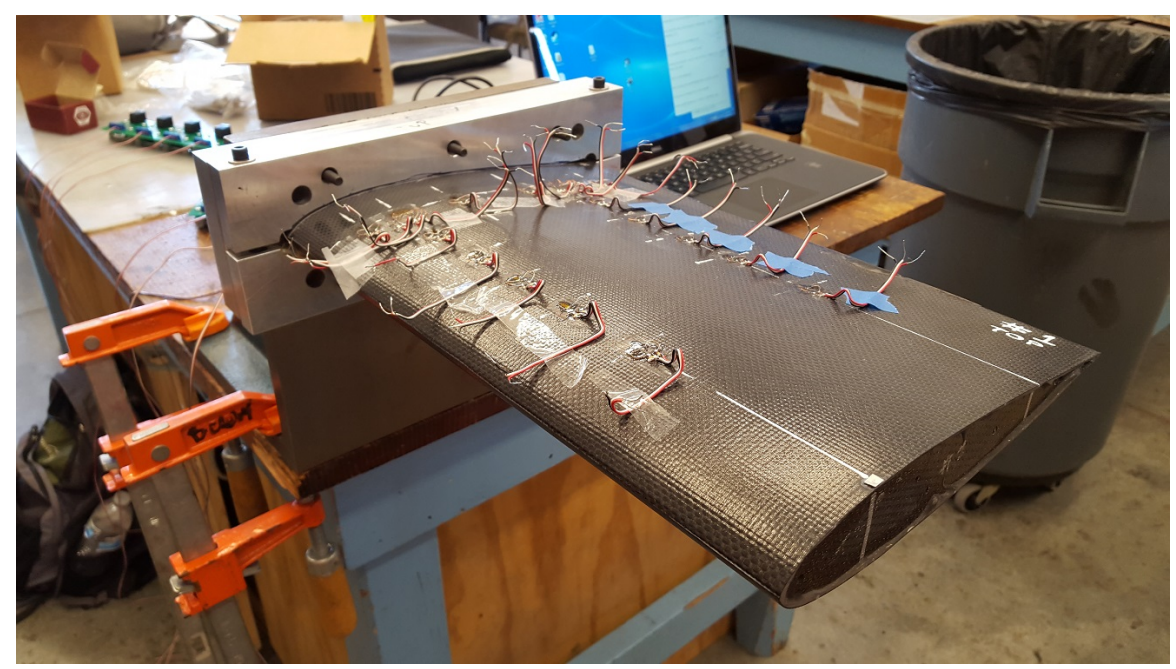

Figure 4.27: Static testing of wing model 
The DAQ is limited to 8 channels therefore each wing had to be tested 3 times to take data from all of the strain gages. Each test was repeated 5 times in order to insure consistent results. The strain gages were divided into groups as shown in Fig.( 4.28) below. Throughout the result sections the strain gages will be defined by the labels shown in this figure. The leading and trailing edge strain gages were placed at a distance of $2.5 ", 4 ", 5.5 ", 7 ", 8.5 "$, and $10.5 "$ from the edge of the root edge. While the chord-wise group was placed at 2.5 inches away from the root edge along a curve from leading to trailing edge. The distances from the nose that the chord-wise strain gages were located were: $1 ", 2 ", 3.5 ", 5.5 ", 6.66 ", 8 "$. In Fig.( 4.29) dimensions are shown between different strain gages mounted on the wing skin.

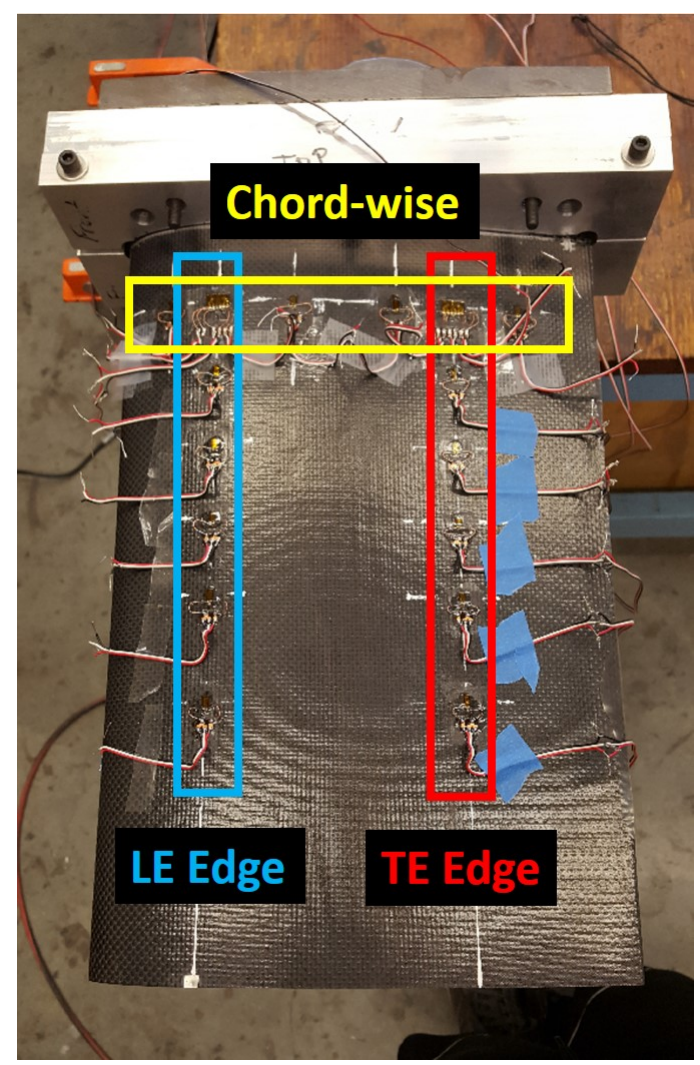

Figure 4.28: Strain gages on the wing model 


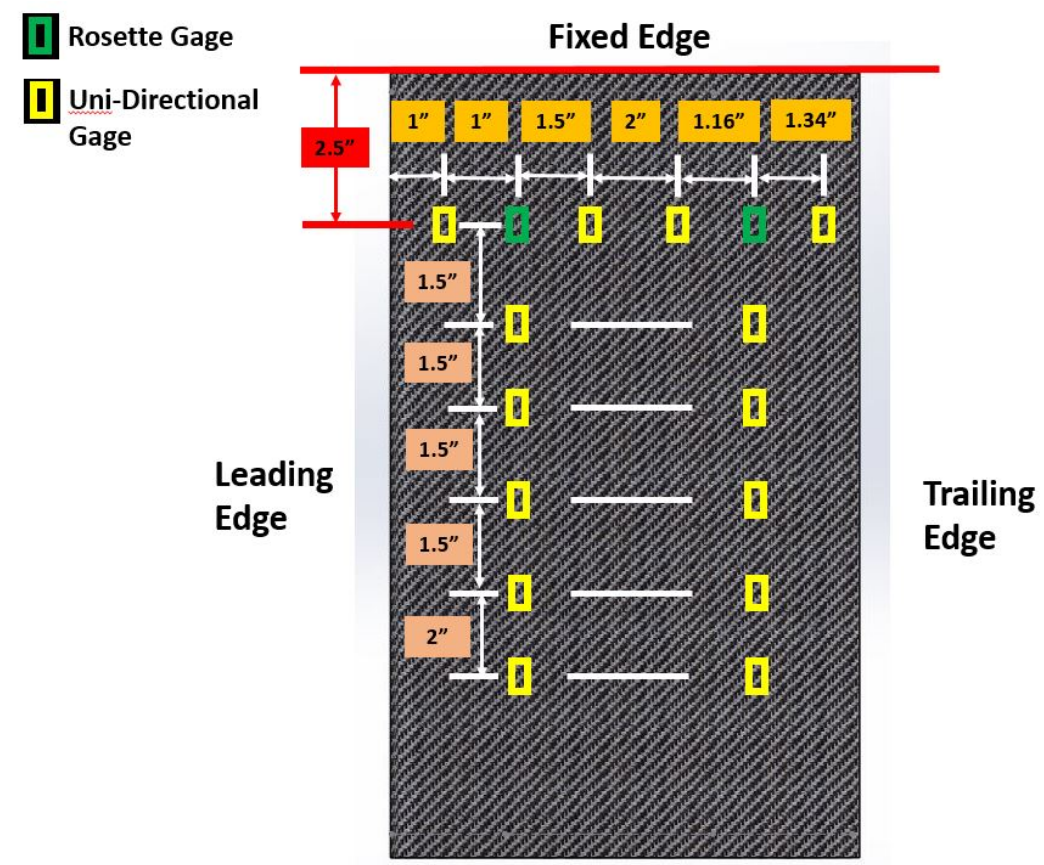

Figure 4.29: Dimensions between strain gages

After the wing and fixture were secured, the wires coming from the RJ-45 breakouts were connected to the lead-wires soldered to the strain gages. After this was done, the setup described in section 2.3 was followed to connect the DAQ to the laptop and start the Strain Smart software. The entire test setup including the laptop, DAQ, test fixture, and wing can be seen in Fig.( 4.30). At this point the strain gages were zeroed, shunt calibrated and data collection window was opened. The start button was pressed so that data would be shown in real time. After this the take data button was pressed to take one data point without any load. Then the weight hanger and weights totaling 2.41 pounds were positioned on the metal tab at the end of the wing. When the data stabilized another data point was taken with load. Finally the load was removed and a last data point was taken to compare to the original tare value. 


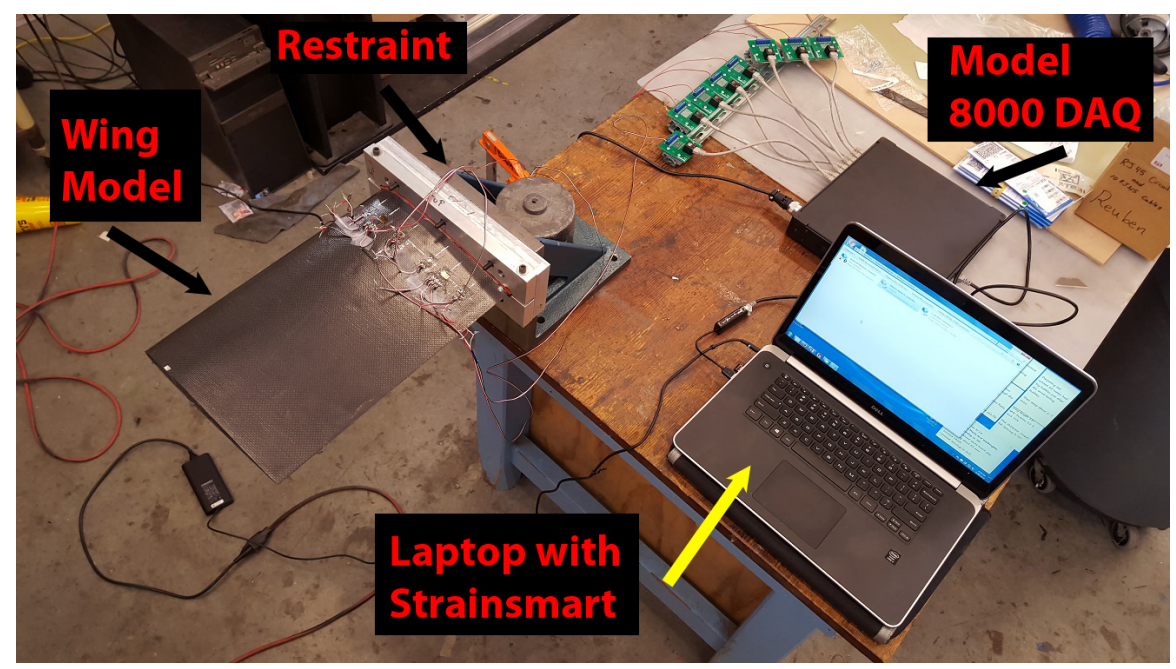

Figure 4.30: Static test setup of wing

It was noticed that there were discrepancies between the initial and final tare values. My hypothesis is that between the time that it takes to record data and balance and stabilize the weight the reading had changed by a small amount. This also could be caused by noise in the data collection or the wing not returning to its initial state after being loaded. To remedy this it was decided to compare the loaded reading with the final reading since they were taken right after each other. This was done for each group of the strain gages 5 times to make sure for consistent data and testing. Each wing was tested in the same manner to check for consistency between different wings manufactured in the same manner. After all the data was taken it was compiled in plots and compared to the FEA results in section 7.2 of this paper.

Since the Instron's actuator is not on the same mounting jaw as the load cell there would not be any force data taken during the fatigue testing. Getting force readings on the wing from the load cell on the top jaw would have required a spring connection from the wing mount to the top grip. In this case the wing would have forces from the spring force as well as the bending. Therefore, based on the elongation of the spring, the force would have been taken out of the load cell 
reading to obtain solely the bending force. Although this was not done because of doubts that this method would have resulted in accurate force readings.

In order to have an estimate of how much force is being applied to the wing a force and displacement study was conducted by static testing on the wing. Weights were added one by one onto the metal hanger and the tip displacement was measured at every load. The displacement was taken with the use of a dial gage and magnetic stand as shown in Fig.( 4.31). When the wing would displace downward under the load, the dial gage probe would elongate allowing for the difference to be measured. The test schematic showing the wing, dial gage, and fixture can be seen in Fig.( 4.32). The plunger of the gage was set adjacent to where the load was concentrated and then zeroed without any load. Once the wing was displaced by a force the plunger would displace with it reading the displacement on the gage. A magnetic stand on a steel block was used, so that the gage would not move after the initial tare was set.

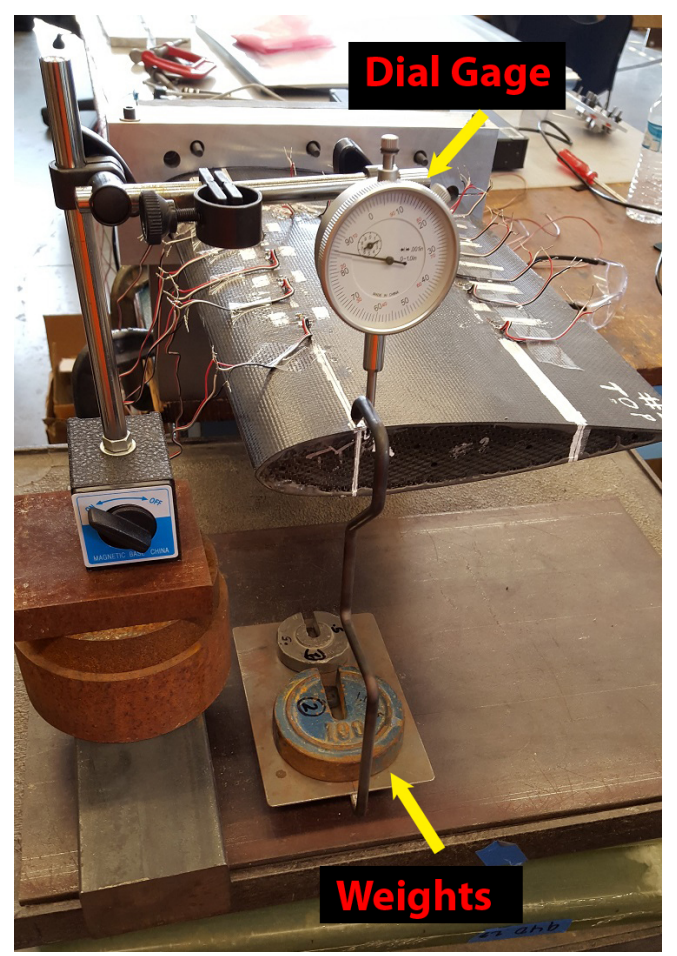

Figure 4.31: Force and displacement test setup 


\section{Dial Gage}

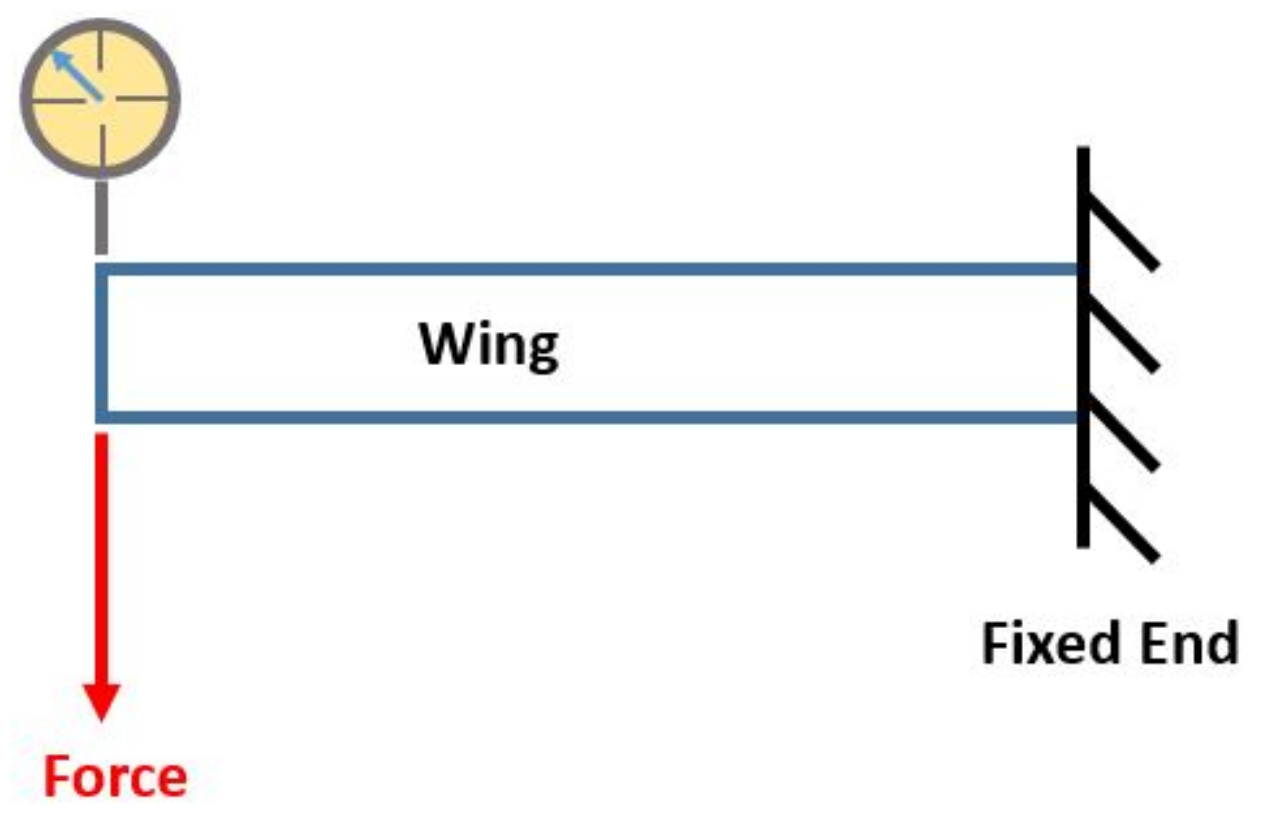

Figure 4.32: Test schematic

A maximum load of $8.27 \mathrm{lbf}$ was added to the wing in order to envelope the displacements that were put on the wing during the Instron fatigue testing. This test was also repeated 5 times in order to make sure that results were consistent with each other. Strain gage data was taken during the test but merely for reference. Following this test, the data was processed so that an estimate for the load being applied by the Instron could be made.

\subsection{Experimental Fatigue Test of the Wing}

In this section the test procedures for fatigue testing was covered first. After this a fatigue behavior study at different test frequencies is covered. Following this a strain behavior study between static and fatigue test outlined. Then the procedures to conduct the fatigue life test were shown. Lastly a test to make sure that no damage had been inflicted to the wings is covered. 


\subsubsection{Experimental Fatigue Test Setup}

Once all of the static testing was completed, it was time to setup the test fixture and wing in front of the Instron machine for fatigue testing. The test setup for all of the fatigue testing can be seen in Fig. (4.33) below. Since connecting the wing mount to the load cell in the top grip would have added complexity and unreliable results, the wing was driven by displacement only. For this reason displacements were placed on the wing rather than forces. The data collected during the fatigue testing was displacement from the Instron control panel and strain from the strain gages. The fatigue testing that was conducted was fully reversed meaning that the displacement on the wing would go positive then negative by the same magnitude then back to the stating point. Throughout this paper this is what would be considered one cycle.

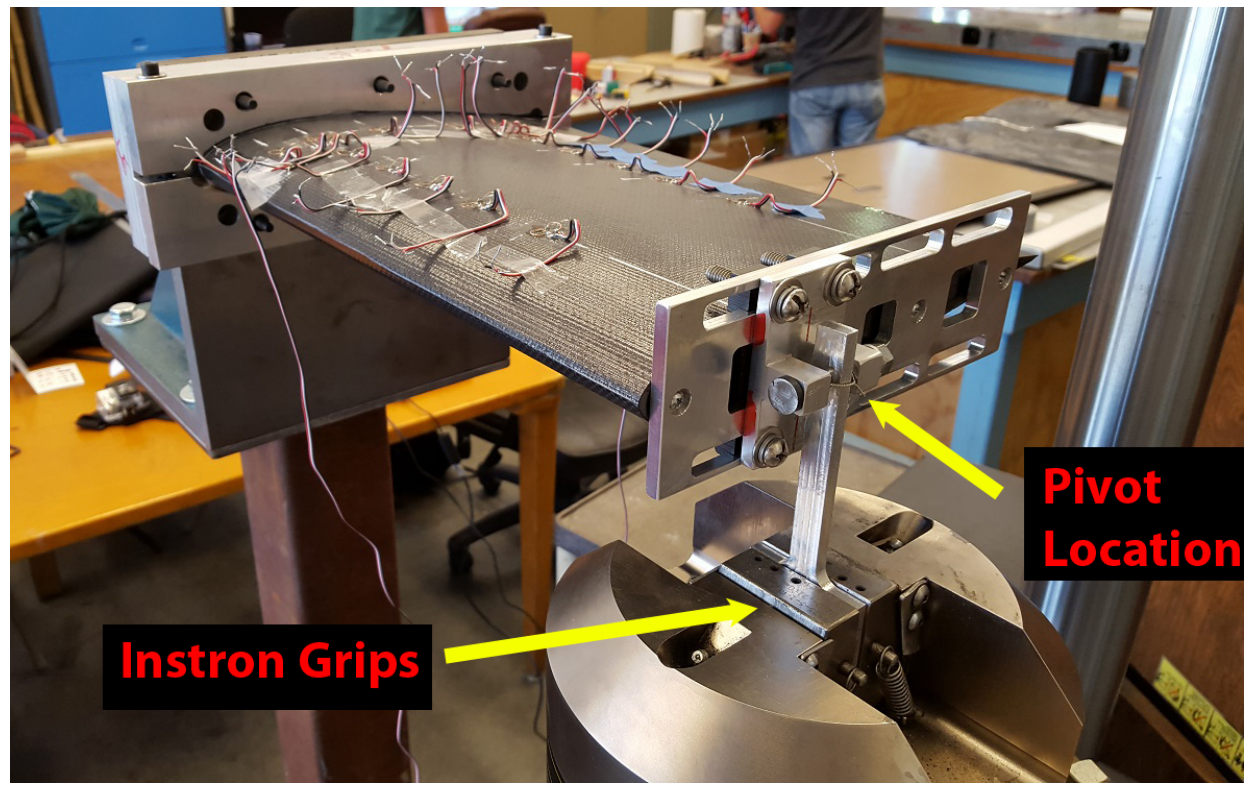

Figure 4.33: Fatigue test setup

It was unpredictable what kind of displacements would be able to be tested with wing. Therefore the starting cyclic displacement placed on the practice wing was decided to be .01 in at $1 \mathrm{~Hz}$. Once it was seen that the wing had no trouble 
with this load it was increased up to .05 in increments of .01 at the same frequency. The wing was not displaced any further than this because of risk of prematurely failing the wing. Additionally conducting a long term fatigue test at this slow frequency would not be ideal.

\subsubsection{Fatigue Behavior at Different Test Frequency}

The first study that was done with the fatigue testing was to determine what frequency the test would be conducted at. This was brought upon since the maximum number of cycles that would be tested for a wing would be 100,000. At this point the test would end since it would be assumed that the wing would not fail from any more fatigue. If a test frequency of $1 \mathrm{~Hz}$ is used then the test will take almost 28 hours, where at a frequency of $10 \mathrm{~Hz}$ it would only take about 2 hours and 45 minutes. Since the machine needed supervision throughout the testing process, a test frequency of $1 \mathrm{~Hz}$ is not feasible. Therefore the goal of this study is to see whether the results from the test change significantly, when changing the rate from $7 \mathrm{~Hz}$ to $10 \mathrm{~Hz}$.

It was learned that at increasing test frequencies the acceleration of the grips could introduce error when using a load cell. This relationship can be seen in Fig.( 4.34) below.[32] Although a load cell would not be used in the wing testing, this curve was used as a guideline. Therefore it was decided to not test at a frequency above $10 \mathrm{~Hz}$. 


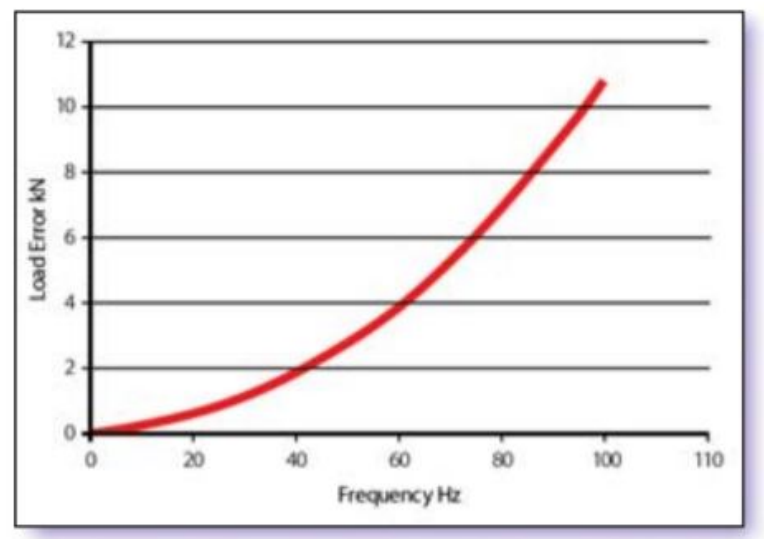

Figure 4.34: Error at different fatigue test frequencies

Cyclic displacements from .01 to .03 were then put on the wing model at test frequencies of 1,7 , and $10 \mathrm{~Hz}$. Data from the one strain gage on the practice wing was recorded to determine if the strains were the same for each displacement at higher test frequencies. If the strains at $10 \mathrm{~Hz}$ were approximately the same as the test done at $1 \mathrm{~Hz}$, then it would be favorable to test at the faster rate. It would take considerably less time to test all three of the wing models at the lower test frequency. In Fig. ( 4.35) the laptop that was used for data collection is shown and the cyclic pattern of the strain can be seen in the screen.

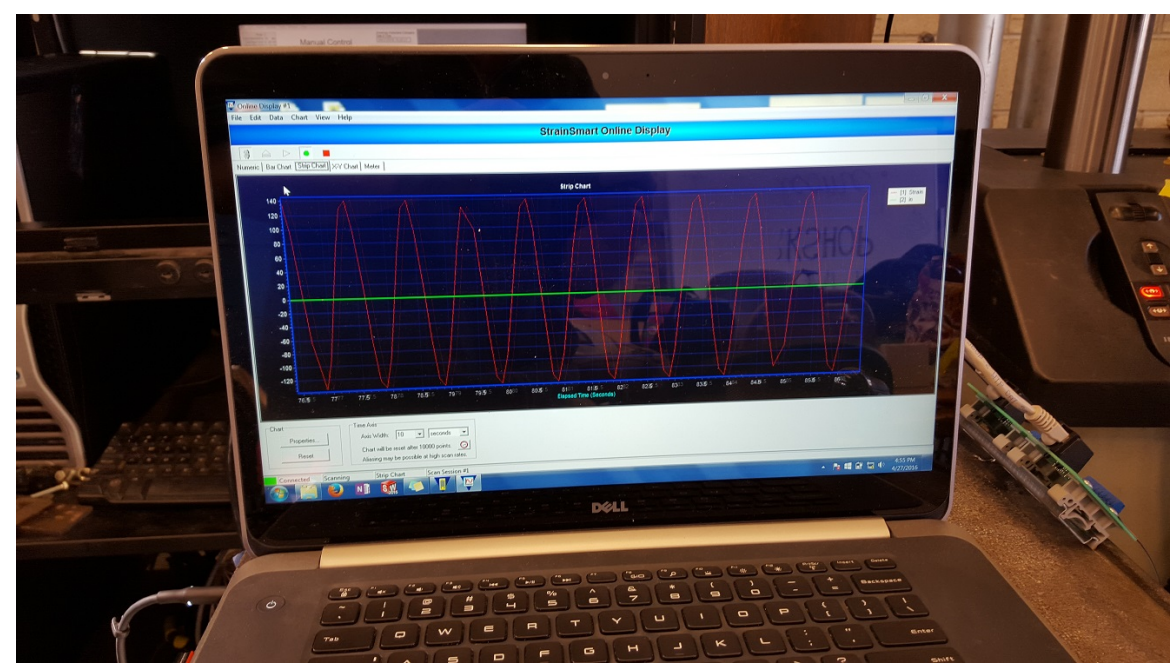

Figure 4.35: Data collection laptop collecting strain data during fatigue test 


\subsubsection{Strain Behavior under Static and Fatigue Loading}

Since the force was not being measured during the test, a way to relate the strain data to some sort of force was needed. It was decided that the strain values from a $1 \mathrm{~Hz}$ fatigue test would be compared to the static testing. Using the strain and force known from the static test and the displacement applied during the fatigue test the fatigue loads could be derived. Each wing was tested from 0.01 to 0.05 inches for 10 displacement cycles at $1 \mathrm{~Hz}$. It was decided to not test past a .05 displacement due to the possibility of premature failure. Data was taken from each strain gage on the wing models for each displacement value. The overall test setup for the fatigue testing is shown in Fig.( 4.36). In this test setup the Instron and its controller can be seen, along with the wing and its fixture, in addition to what was used for data collection which included the DAQ and the laptop. Since in static testing it was found that the leading edge strain gages were most reliable, these would be compared between the two different types of test. Depending where the strain value lied on the strain and cyclic displacement curve the force could be estimated for the fatigue testing.

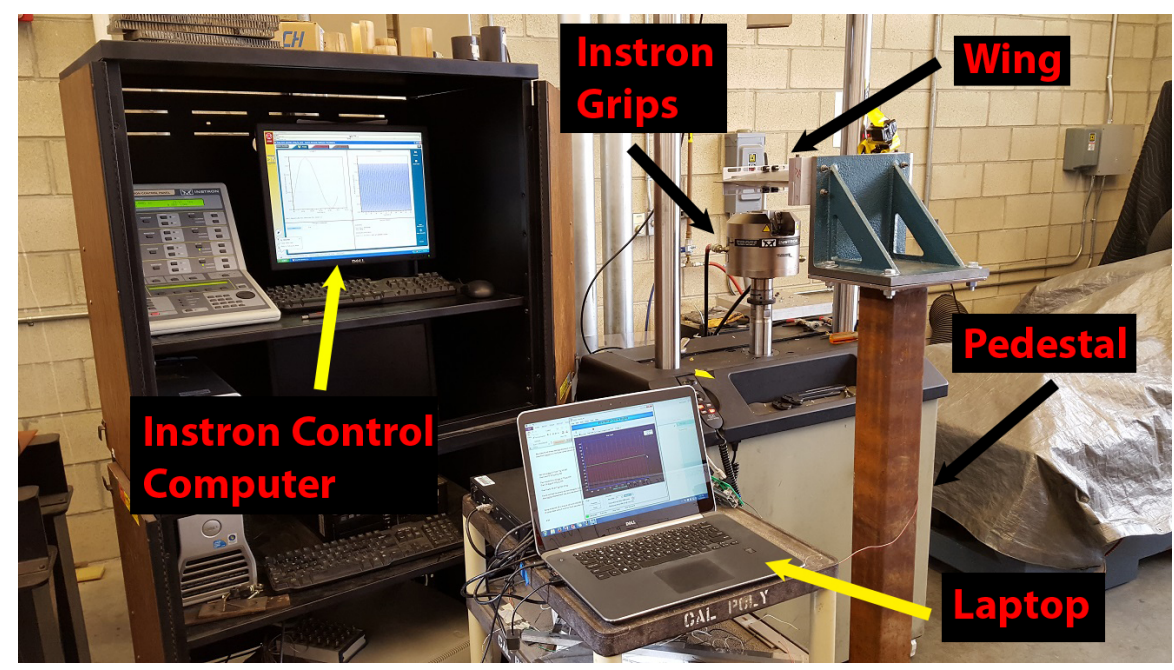

Figure 4.36: Fatigue test setup including Instron, wing, fixture, DAQ, latop 


\subsubsection{Fatigue Life Test}

The last type of fatigue study was the conventional long term test where the specimen would be cycled till failure or 100,000 cycles, whichever came first. The wing would be tested to a displacement amplitude of .03 at frequency of $10 \mathrm{~Hz}$. In Fig.( 4.37) the actual fatigue cycle that will be used for the test can be seen.

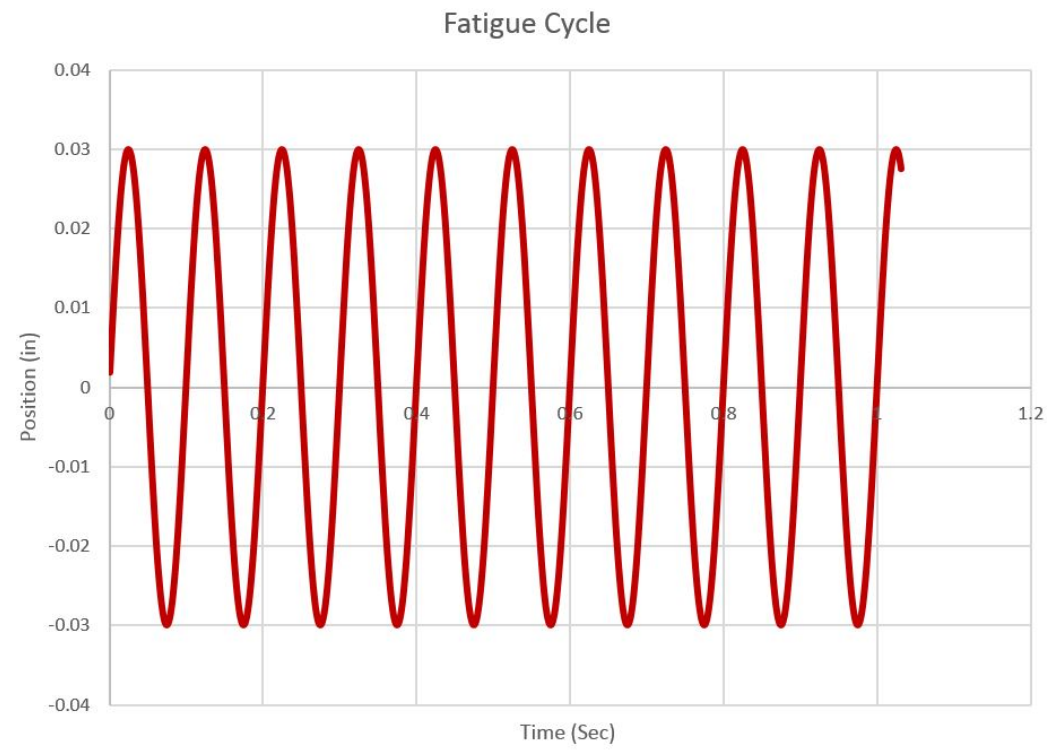

Figure 4.37: Position controled fatigue cycle

The cyclic displacement was clearly visible when standing next to the machine and it was apparent that it was applying a moderate load to the wing. Carbon fiber tends to have good fatigue properties so a failure in the part is not expected. However after so many cycles, it was expected that the joints or bonds would begin to fail. Throughout the test the fixture and base did not vibrate to the point where the data was affected or error was introduced it. None of the bolts and screws came loose after the almost 3 hour test of repeated cycles. 


\subsection{Static Test after Fatigue Test}

Composites can be damaged without showing any signs of fracture on the surfaces. Therefore it was decided to test one wing model under the same static loading as before to insure that the strains had not changed after the fatigue test. If the wing model had been damaged internally the strains from the static test should be different than the initial testing. A small difference in strain results is allowable since there is variability of mounting, and test conditions. The wing models were retested following the exact same procedure as the initial static testing. Only the leading edge strain gages were to be compared before and after the test since they were the most reliable. 
Chapter 5

EXPERIMENTAL RESULTS AND DISCUSSION

In this chapter all of the experimental results were discussed. The first results that were investigated were the material properties. Afterwards, the results for the metal and composite cantilever beams are covered and compared to theoretical

calculations. A study was conducted to determine if changing the boundary condition of the wing would change the results. Next the strain measurements on the wing skin are discussed for the three locations. The next section covered results for the fatigue behavior at different test frequencies. Then the strain results from the static and fatigue loadings are shown. The outcome of the fatigue life test of the wing models is discussed. Lastly, the results of the post-fatigue testing were explained which would show if any damage occurred.

5.1 Material Property Results

In this section, the results obtained for the composite materials used in the project are covered. The first subsection covers the tensile testing where the ultimate strength and modulus were measured. Data is shown for the different types of measurement devices used in the testing. In the next subsection, the volume fraction results are covered and discussed.

\subsubsection{Uni-axial Test Results}

After the data from the material testing was collected, it was imported into an Excel file so that it could be post-processed. The key properties that were 
looked at in this testing were modulus, ultimate strength and Poisson's ratio. The percent error was calculated between the experimental results and the material's data sheet.

In order to insure that the testing procedures were reliable an aluminum specimen with two strain gages was tested. The specimens did not yield since it was only loaded to $25 \%$ of its ultimate strength. After testing the modulus it was found to be $10.3 \mathrm{Msi}$ and 9.9 Msi using the strain gage and extensometer respectively. The corresponding errors for these values compared to the data sheet were $3.0 \%$ and $0.7 \%$ which shows that the testing is accurate. The measured Poisson's ratio for the sample was found to be 0.327 , which was only $0.9 \%$ away from the data sheet value. These results and errors between the experimental test and data sheet for the aluminum specimen are shown tabulated in Table ( 5.1).[33]

Table 5.1: Summary of aluminum sample material properties and percent errors from data sheet

\begin{tabular}{|l|l|l|l|}
\hline & Experimental & Data Sheet & Percent Error \\
\hline $\begin{array}{l}\text { Tensile Modulus - } \\
\text { Strain Gage (Msi) }\end{array}$ & 10.3 & 10.0 & 3.0 \\
\hline $\begin{array}{l}\text { Tensile Modulus - } \\
\text { Extensometer (Msi) }\end{array}$ & 9.9 & 10.0 & 0.7 \\
\hline Poisson's Ratio & 0.327 & 0.33 & 0.9 \\
\hline
\end{tabular}

In order to determine the tensile modulus of the composite tensile samples the strain was measured between $25 \%$ and $50 \%$ of the materials ultimate tensile strength. This was done to minimize the damage introduced into the sample during testing. One sample was tested without any strain measurement devices till failure, to determine its tensile strength. In Fig.( 5.1) the stress-strain curve of one of the failure samples is shown. As expected, for a composite material, the failure was catastrophic. Composite materials do not yield therefore they experience sudden failure.

Then two samples with strain gages were tested, along with an extensometer 
for comparison. The strain data measured was then plotted with the stress so that the modulus could be calculated. It was found that the extensometer and strain gage yielded similar modulus calculations. Since the extensometer was easier to utilize than the strain gages, it was used for the rest of the samples. Samples with the extensometer had the test paused at $50 \%$ of its strength and the extensometer was removed, and then test was continued until failure. This process was repeated to obtain the modulus and strengths for both materials.

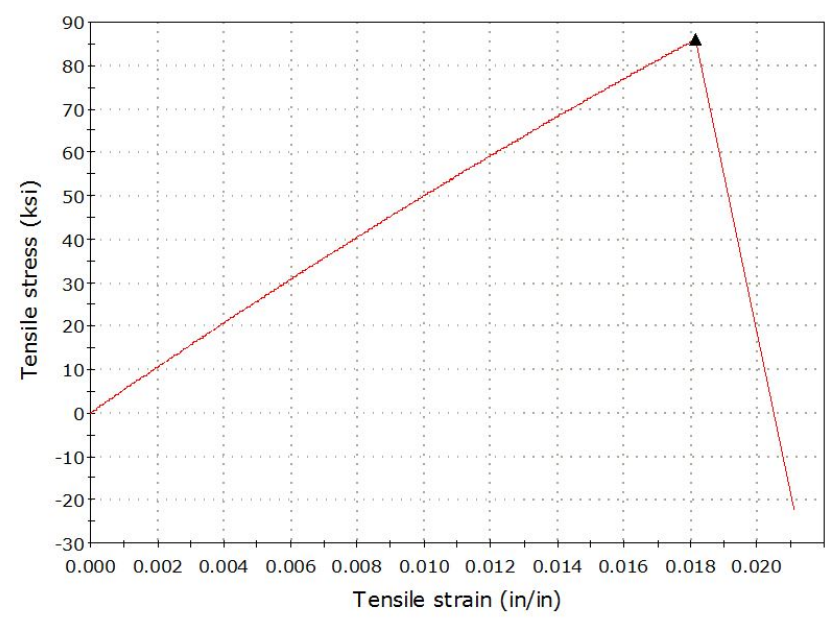

Figure 5.1: Stress stain curve of a failed composite tensile specimen

The first composite material that was tested was the Cycom weave. The specimens were 1 in $\mathrm{x} 10$ in and consisted of 10 layers of 0 degree composite fabric. The calculated tensile modulus using the extensometer and strain gage were $6.7 \mathrm{Msi}$ and 6.5 Msi respectively. Both of the curves for each of the strain measurement devices are shown in Fig.( 5.2). The errors associated with those values compared to the data sheet were $4.5 \%$ and $7.1 \%$ respectively. These errors are low considering that the material was donated and very well could be expired or defected. 


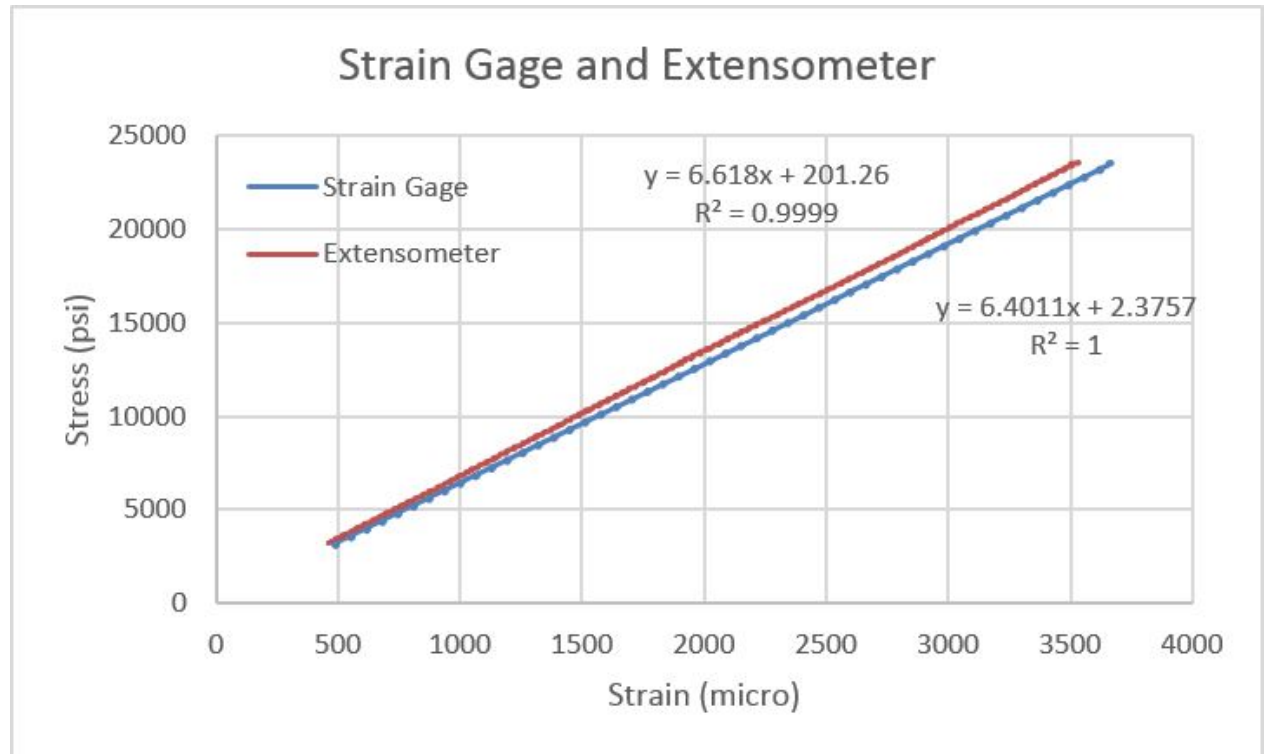

Figure 5.2: Extensometer and stain gage stress - strain curves

In Fig. ( 5.3) it can be seen from the stress-strain curve that the composite specimens suffered a catastrophic failure which is expected from a composite material. Note that the curve changed because before this point the displacement was being measured with the extensometer, and after it was read by the Instron actuator. The reason for this was to be able to remove the extensometer before specimen failure which would damage the extensometer. The erratic signal at the end was due to the load cell measuring the vibrations due to the failure of the specimen.

The failure tensile stress from the tested specimens was $83.5 \mathrm{ksi}$ which was higher compared to the data sheet by $28.5 \%$. A possibility is that there was more fiber content making it stronger in the axial direction. Many parts made with this material were found to have some epoxy starved areas from observation. Each specimen tested had a similar trend showing that they were made and tested in a consistent manner. The material was also donated, therefore it could have been mishandled or expired. In Table ( 5.2) the comparison of the experimental and data sheet properties is shown. 


\section{Specimen 1 to 4}
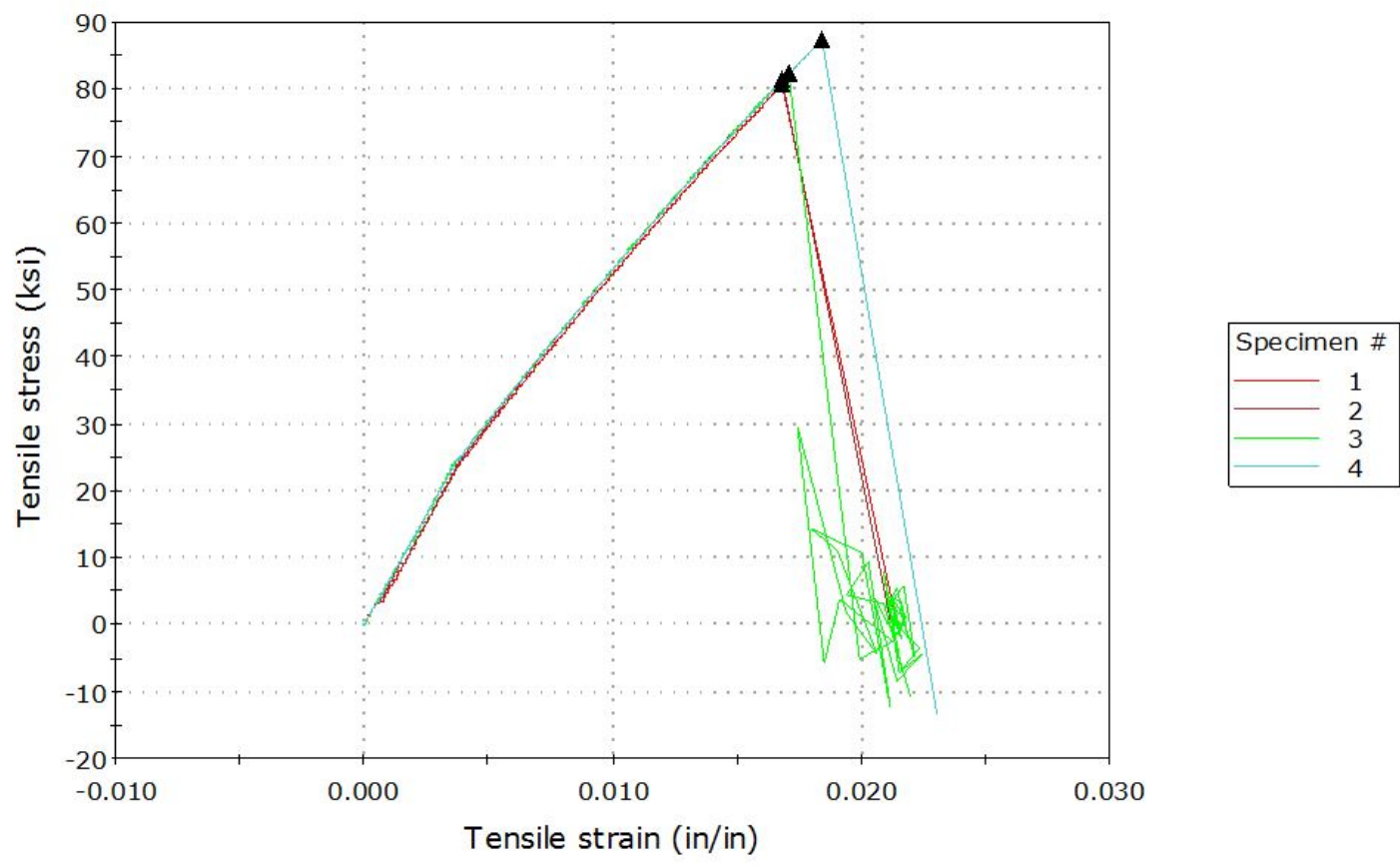

Figure 5.3: Stress stain curve of the Cycom specimens tested until failure

Table 5.2: Summary of Cycom tensile material properties and percent error from data sheet

\begin{tabular}{|l|l|l|l|}
\hline & Experimental & Data Sheet & Percent Error \\
\hline $\begin{array}{l}\text { Tensile Modulus - } \\
\text { Strain Gage (Msi) }\end{array}$ & 6.5 & 7 & 7.07 \\
\hline $\begin{array}{l}\text { Tensile Modulus - } \\
\text { Extensometer (Msi) }\end{array}$ & 6.7 & 7 & 4.5 \\
\hline Tensile Strength (ksi) & 83.5 & 65 & 28.52 \\
\hline
\end{tabular}

The next composite material that was tested was the Hexcel weave that would be used for the ribs. A plot of multiple test specimens that were tested can be seen in Fig.( 5.4). The calculated tensile modulus using the extensometer and strain gage were 16.2 Msi and 16.4 Msi respectively. The tensile modulus reported in the data sheet for this material was $9.7 \mathrm{Msi}$. The errors associated with those values compared to the data sheet were very high. This could have been because 
of the material being defected or resin loss due to improper storage. If resin is lost from the pre-impregnated material the cured part is likely to be resin starved increasing its strength because there is a larger percentage of fiber to carry the load. Since both methods to measure displacement reached a very similar result for the modulus it shows high confidence for the test result. These samples were tested in the same manner as the aluminum and Cycom samples that had very little error from the data sheet.

\section{Specimen 1 to 4}

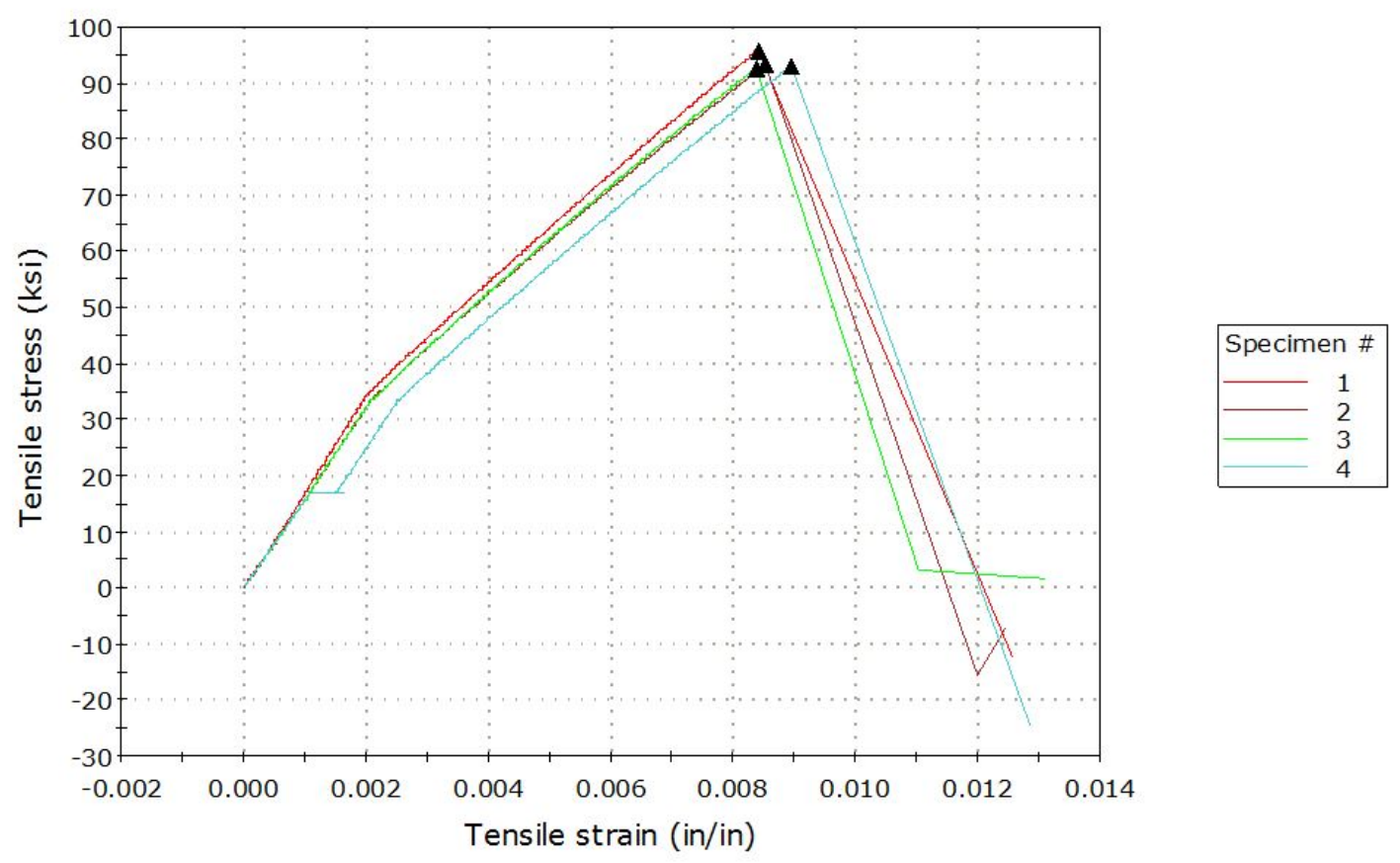

Figure 5.4: Stress stain curve of the Hexcel specimens tested till failure

The specimens of this material had the same type of failure mode as the Cycom material which is expected since they are both composite weaves. All samples followed a similar stress-strain curve and failed at about the same point. The average failure stress of the specimens was $94.7 \mathrm{ksi}$, which was less than the data sheet by $34.1 \%$. This could most likely be attributed to the material quality. Prior test have shown that the test procedures deliver consistent and reliable results. A 
summary of the percent errors compared to data sheet are shown in Table ( 5.3).

Table 5.3: Summary of Hexcel tensile material properties and percent error from data sheet

\begin{tabular}{|l|l|l|l|}
\hline & Experimental & Data Sheet & Percent Error \\
\hline $\begin{array}{l}\text { Tensile Modulus - } \\
\text { Strain Gage (Msi) }\end{array}$ & 16.4 & 9.7 & 40.9 \\
\hline $\begin{array}{l}\text { Tensile Modulus - } \\
\text { Extensometer (Msi) }\end{array}$ & 16.2 & 9.7 & 39.9 \\
\hline Tensile Strength (ksi) & 94.7 & 127 & 34.1 \\
\hline
\end{tabular}

The Poisson's ratio for the Cycom and Hexcel materials were found to be .04 and .015 respectively. These values are very low compared to the aluminum since composites do not deform much in the perpendicular direction to the load. Homogeneous materials on the other hand neck inwards at loads even lower than there yield. The data sheets for each composite material did not show a reference Poisson's ratio therefore a comparison to the experimental value was not possible. The Poisson's ratio for each material was used in the FEA model so that the material would be simulated accurately. In Table ( 5.4) below the Poisson's ratios for each of the three materials.

Table 5.4: Measured Poisson's ratio for both composite materials used

\begin{tabular}{|l|c|}
\hline Material & Measured Poisson's Ratio \\
\hline Cycom & 0.040 \\
\hline Hexcel & 0.015 \\
\hline
\end{tabular}

\subsubsection{Fiber Volume Fraction Results}

All of the data was recorded and imported into a Matlab file so that post processing could be done. A function was made that would calculate the volume fraction of each specimen based on its weight loss, which should have been only epoxy. The densities used for the matrix and laminate used in the calculations for the Hexcel material came from the data sheet. The laminate density was not 
shown in the Cycom data sheet therefore it had to be calculated based on the sample dimensions and mass. However the Cycom resin density was available and used in the calculations.

The theoretical fiber volume fraction of the Hexcel material from the data sheet was $55.3 \%$. The average volume fraction found from the test specimens was $56 \%$. One of the specimens was excluded from the average since it was an outlier. The average measured ratio differed from the data sheet by only $1.2 \%$ which showed that the curing process is similar to the manufactures. This therefore did not give much insight into why the stiffness of the specimens was considerably higher than the data sheet. The thickness of our specimens were thicker which might have had an affect on this value. Since the fibers are optimized to resist the axial loads it was expected that the material would have had a higher fiber ratio than the data sheet but this was not the case. The results for each specimen and the differences to the theoretical value can be seen in Table ( 5.5$)$.

Table 5.5: Summary of the Hexcel volume fraction results

\begin{tabular}{|c|c|c|c|}
\hline \multicolumn{4}{|c|}{ Hexcel Fiber Volume Fraction } \\
\hline Sample \# & Measured (\%) & Data Sheet (\%) & Percent difference (\%) \\
\hline 1 & 43.2 & 55.3 & 22.0 \\
\hline 2 & 58.3 & 55.3 & 5.5 \\
\hline 3 & 53.6 & 55.3 & 3.0 \\
\hline Average & 56.0 & 55.3 & 1.2 \\
\hline
\end{tabular}

The theoretical fiber volume fraction of the Cycom fabric was listed as $55 \%$ in its data sheet. The average volume fraction calculated for the test specimens was 49.3\%. This value differed from the reference value by $10.5 \%$ which is not a considerable amount. Since the fiber ratio was lower in our specimens the conclusion can be made that it is resin rich. This can be caused by not enough pressure being applied to the laminate during curing. The role of pressure in the curing process is to remove the excess epoxy from the part and for it to take the shape of the 
tooling. The data for each test specimen for this material can be seen in Table ( 5.6) along with the differences from the theoretical value.

Table 5.6: Summary of the Cycom volume fraction results

\begin{tabular}{|c|c|c|c|}
\hline \multicolumn{4}{|c|}{ Cycom Fiber Volume Fraction } \\
\hline Sample \# & Measured (\%) & Theoretical (\%) & Percent difference (\%) \\
\hline 1 & 49.5 & 55.0 & 10.0 \\
\hline 2 & 48.1 & 55.0 & 12.5 \\
\hline 3 & 50.4 & 55.0 & 8.4 \\
\hline Average & 49.3 & 55.0 & 10.5 \\
\hline
\end{tabular}

Another source of error in this calculation could have been the scale. It was seen to deviate from its nominal reading at times. It could also have been attributed to not all of the epoxy being burned off in the oven. If the specimen were pulled before all the epoxy were burned, there would be considerable error in the measurement. This kind of error would cause the specimen to have less fiber than expected. By visual inspection it was seen that some of the parts made with the Cycom material looked epoxy starved. Although the test results prove otherwise which could have been caused by the surface of the material lacking epoxy but the inside layers being resin rich. I had expected for the volume fraction of this material to be fiber rich based on visual inspection of the parts. The tested stiffness of this material did match the data sheet so this could have led us to believe that the fiber volume ratio of our specimens would have matched the data sheet.

\subsection{Experimental Static Test Results}

In this section different results for the static testing are covered. In the first sub section the results from studies used to validate the FEA model are covered. These studies consisted of applying bending loads to cantilever beams made of different materials. The next section covered how changing the boundary condition of the wing would affect the static bending test. Lastly, the strain results from the static 
bending test of the wing models are discussed.

\subsubsection{Validation Test Results}

After the metal cantilever beam was tested, the strain measurements were exported out of the DAQ software into an Excel spreadsheet. The strain at the 4" and 8" locations were measured to be $-172 \mu \epsilon$ and $-139 \mu \epsilon$ respectively. This trend was expected since the highest strain for a cantilever beam is at its fixed end. After calculating the theoretical strain at each location, they were found to be $-159 \mu \epsilon$ and $-129 \mu \epsilon$ respectively. The difference between the experimental test and theoretical calculation was $7.7 \%$ and $7.5 \%$ respectively for each strain gage. In Table (5.7), a summary and comparison of the experimental test results and the analytical calculations are shown.

Table 5.7: Comparison of experimental and analytical strain values of aluminum beam

\begin{tabular}{|l|c|c|c|}
\hline Strain Gage $\#$ & EXP $(\mu \epsilon)$ & THEO $(\mu \epsilon)$ & Percent Error $(\%)$ \\
\hline 1 & -172.0 & -159.7 & 7.7 \\
\hline 2 & -139.0 & -129.3 & 7.5 \\
\hline
\end{tabular}

The strains from the analytical solution were lower than the experimental test, which is usually the case since there are external factors in the latter. In reality the material tends to have flaws causing it to be weaker than the theoretical value. This would in turn allow a beam to deflect more and read a higher strain than a theoretical calculation. This explains why the experimental test yielded slightly higher strains than the calculation.

Additionally, in an experimental test, the load might not have been loaded in the exact location as the analytical calculation. Also, it might not have been loaded exactly in the center, which would have added a torsion load. A plot of the analytical equation assumes that beam is under pure bending and the loads are exactly where intended. 
The experimental test also was done with a beam that has been in the lab for many years and could have degraded its material stiffness which would cause the higher strains measured. The strain gages that were measured were also many years old and have experienced different weather conditions, that could affected their accuracy. The last possible source of error could have been the gage factor for the measurement since the strain gages were applied many years ago it was assumed to be 2 . Similar beams made of the composite materials were tested and covered next.

After the testing the cantilever beams made of both materials, the data was processed in Excel to show the strains across the beam length. It was found that the strains were the highest at the constraint and gradually decreased toward the free end. These results follow the expected trend for a cantilever beam under a load at its free end. This shows that this test is most likely constrained properly. After calculating the theoretical strains using an analytical calculation it was found that the Cycom material matched fairly accurately. The beam made of Hexcel material did not match accurately as the beam made of the Cycom material.

The results from both cantilever beams can be seen in Fig.( 5.5). In the Cycom results plot on the left, it can be seen that at the fixed end the difference between the data was greater than the free end. When the percent differences at each strain gage location were averaged it was found to be only a $8.5 \%$ difference. For the Hexcel cantilever beam the results differed considerably with an average error of $35 \%$. 


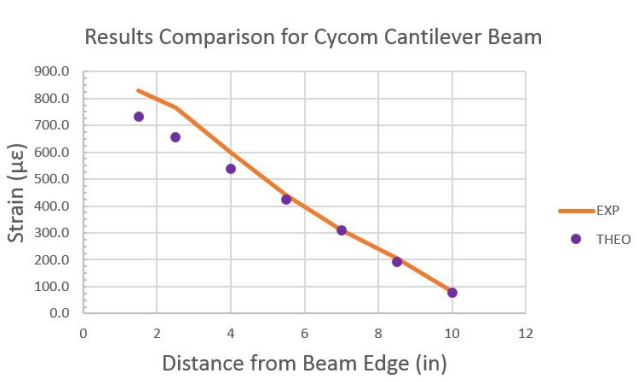

(a) Cycom material results

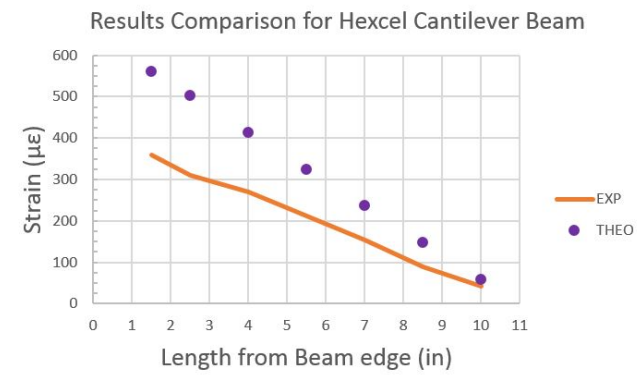

(b) Hexcel material results

Figure 5.5: Carbon fiber strain distribution along beam

The reason that the Hexcel results differed by so much was because the theoretical calculation was done with the modulus from the data sheet. Although, from the material testing, it was found that the the test samples were about $40 \%$ stiffer than expected. It can be seen that additional stiffness in the cured samples affected the strain results by a similar proportion. The cured material was stiffer therefore resulting in lower strains than the analytical calculation which used the data sheet stiffness. Some error can be explained by using standard super glue to bond the strain gages rather than the adhesive recommended by the manufacture.

After this, an FEA model was made of the cantilever beam so that it could be compared to the experimental results. The experimental material properties were inputted in the FEA software, so that it could be compared to the experiment. If both types of methods reach similar values for strains then it would give confidence in the experimental material properties, FEA model boundary conditions and testing methods. This would also show that the strain gages are showing realistic results, and that the strain values from the wing models would be trustworthy. The schematic of the cantilever beam test can be seen in Fig.( 5.6). 


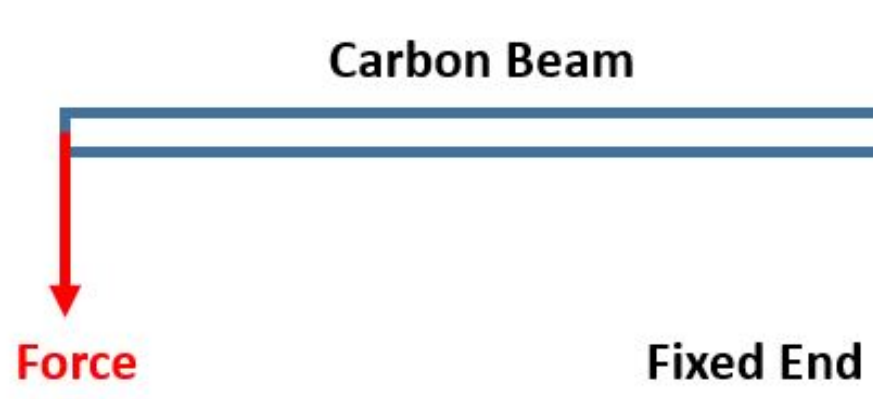

Figure 5.6: Schematic of carbon fiber beam test

\subsubsection{Adjusted Fixture Constraint Results}

It was noticed that because of damage in the area of the wing skin mold, there was a bump on one side of the wing model. Due to this defect, the wing model did not sit flush on the clamping fixture. The concern about this is that the wing would not be properly constrained and would change the strain results from the strain gages. If the wing is allowed to move in this area then the strains would be lower than anticipated.

In order to see if this problem could be fixed, epoxy was used to fill this gap. Release treatment was put on the metal fixture so that epoxy could be removed after testing. After testing the same wing with and without the epoxy, it was found that it did not affect the results greatly. The trailing edge strains did decrease compared to the one with the carbon fiber spacer. This could have been because of dampening of the epoxy compared to a stiff material such as the spacer. Dampening in this area is not how the boundary condition was intended to be. For this reason it was decided to carry on and test the rest of the wings without adding epoxy. A carbon fiber spacer was used to add a load path near the trailing edge spar. In Fig.( 5.7b), Fig.( 5.8a), and Fig.( 5.8b) the comparisons between with and without epoxy can be seen for the leading edge, trailing edge, and chord-wise strain gages respectively. 


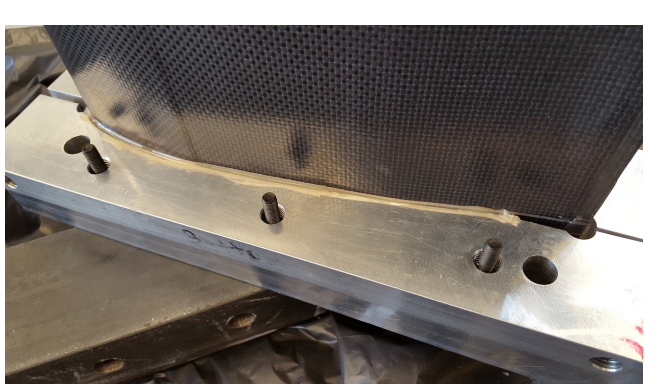

(a) Cured epoxy used to fill boundary condition void

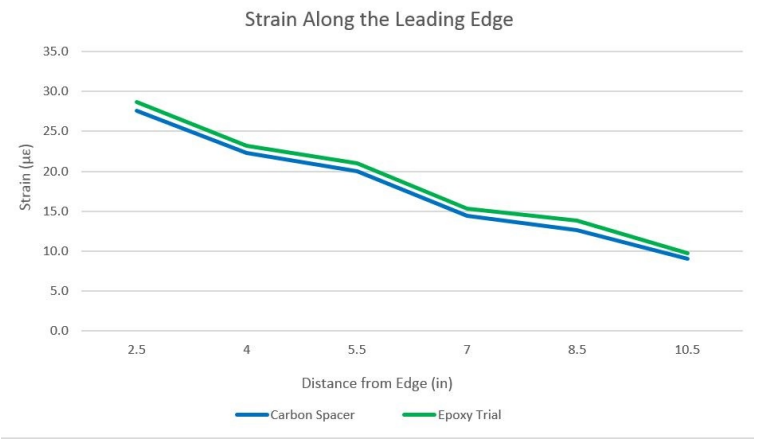

(b) Leading edge comparison results

Figure 5.7: Boundary condition filled in with epoxy and comparison results for leading edge strain gages

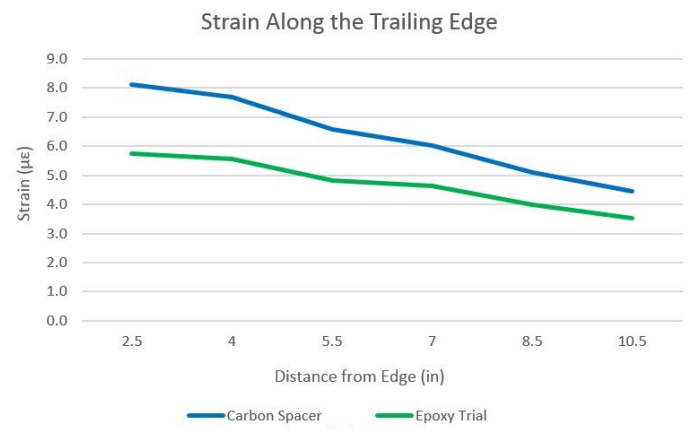

(a) Trailing edge comparison results

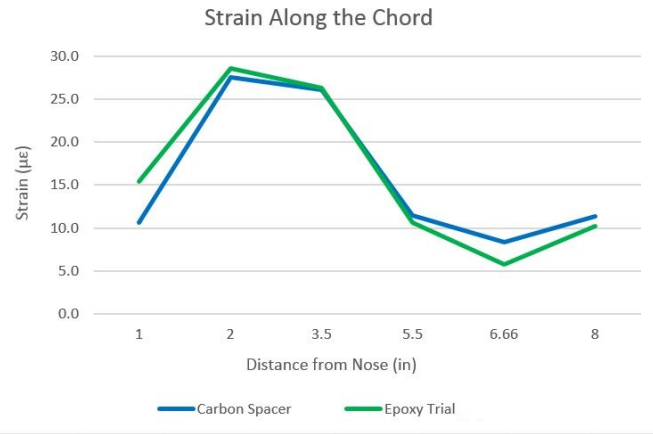

(b) Chord-wise comparison results

Figure 5.8: Comparison of carbon spacer and epoxy boundary condition adjustment

\subsubsection{Wing Static Test Results}

After all the data for the three groups of strain gages on the wing skin was collected, it was then complied in an Excel spread sheet for organization. It was found that the testing was very consistent after loading and unloading the weight 5 times for each strain gage group. Even when a wing was unmounted from the test fixture and retested in the same configuration, the values from the strain gages were nearly identical. This study was done in order to be compared to the FEA model for validation. Therefore it is desirable for the results from the three wings to be consistent. 
The first group of strain gages that were tested for each wing were the ones directly above the leading edge spar. It was seen that the strain gages closest to the fixed side read the highest strain and decreased in magnitude, closer to the wing tip. This was expected since the wing was tested as a cantilever structure with a point load at the end. All three wings showed this trend for their respective leading edge strain gages. Between the three wing models, there was only a small difference in strain between the corresponding strain gages along the wing shown in Fig.( 5.9). Which showed that the manufacturing and assembly methods allowed for similar characteristics in this area.

Comparison of Leading Edge Experimental Data

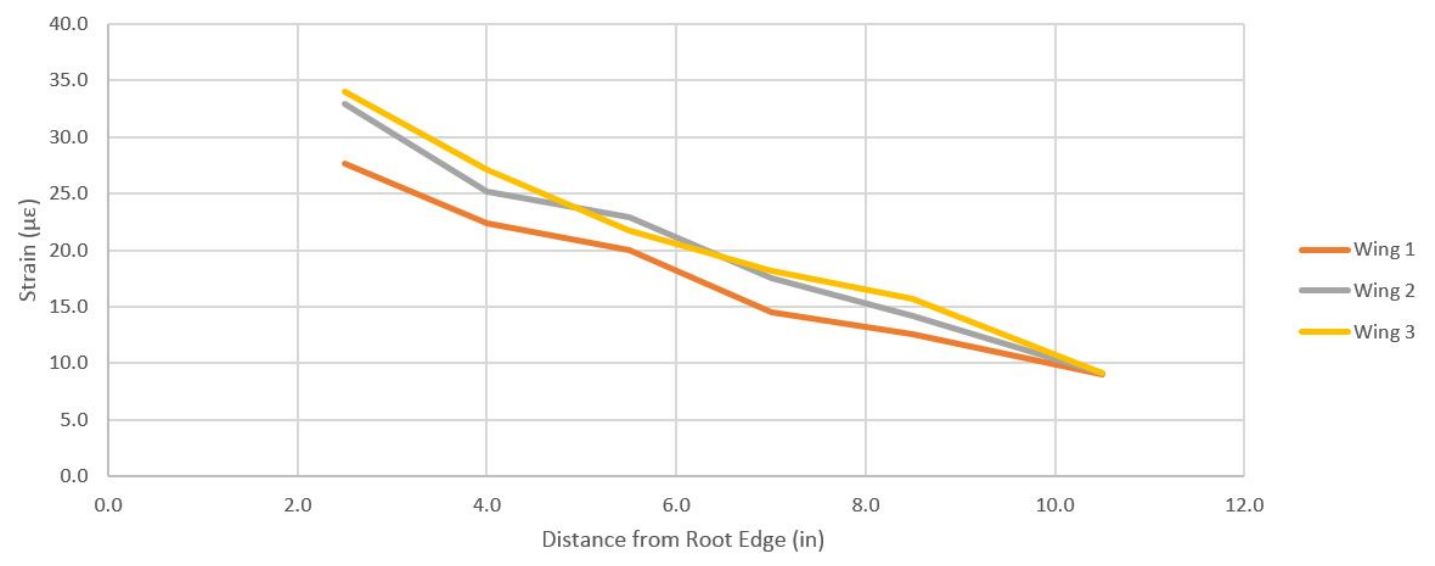

Figure 5.9: Strain values from the three wing models along the leading edge spar

The next group of strain gages which were tested were the ones located along the trailing edge spar. This group also had consistent strain results after being loaded and unloaded for each each wing model. Although the results between the multiple wings were not in agreement, wings one and three were found to have similar strain values along the spar, but wing two had strains that were twice as much in magnitude. The inconsistency could be attributed to the manufacturing process or possibly the boundary condition issue which was explained in the last subsection. The strain gages did show a diminishing strain from the fixed side to the free end, but it was not as apparent as the leading edge spars. 
Since structural adhesive was used for bonding different parts together, there might have been an inconsistency of how much adhesive was used between different models. A possible solution for this would have been to use an adhesive film so that a consistent bond line would be between the spar and the wing skin. A possible solution to solve the boundary condition issue would have been to use a solid aluminum mold, since the damage occurred when bonding the metal sheet onto the foam mold. The comparison can be seen in Fig.( 5.10) where the strains for the three wings are plotted against their distance away from the root edge.

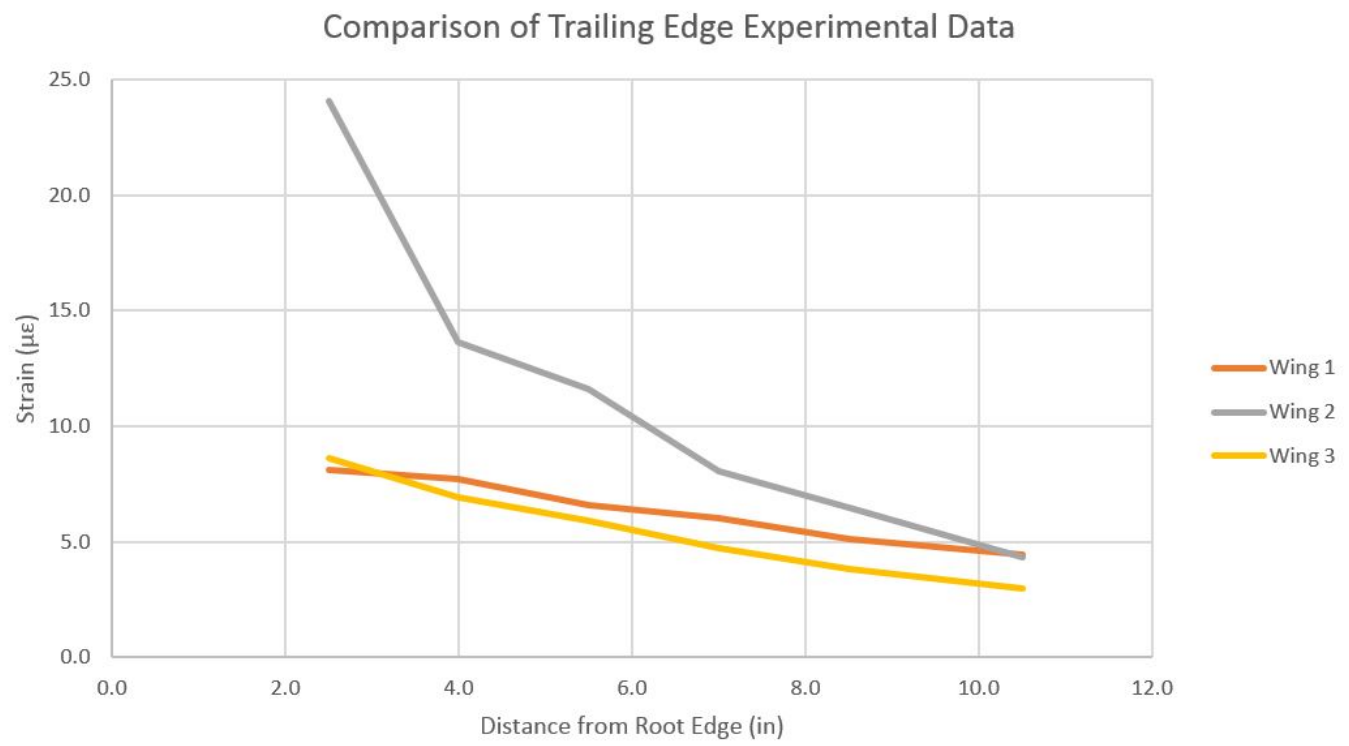

Figure 5.10: Strain values from the three wing models along the trailing edge spar

The last group of strain gages were in the chord-wise direction. Two of these strain gages were included in the leading and trailing edge groups. Since they were covered earlier in this section, emphasis is on the other four strain gages. Two other strain gages were placed on the outside of the spars towards the leading and trailing edge of the wing. The last two strain gages were placed between the spars. The trend that was expected for the chord-wise group was for the stress to be highest near the leading edge spar and then gradually decrease towards the wing's trailing edge. 
Looking at the strain gage on the leading edge of the model, wing two and three had similar results but wing one differed by a significant amount. The strains were also lower than expected, which could be because the strain gage would be on a curved surface in this area. The curved shape for the leading edge and could have been stiffer than what the FEA model simulated. Since the strains were low between the spars it could mean that this area was flexing under load. The strain gage just inside of the leading edge spar had wings two and three match but wing one showed a much greater strain than the latter. This lack of trend of data could mean that these locations were more susceptible to orientation of the strain gage and placement. Since they would all have to follow an exact strain line along the chord and any misplacement would throw off the smooth curve.

The spar strain gages on the other hand, if misplaced along the spar would still show a decreasing trend which was seen. The next strain gage closest to the trailing edge spar was in better agreement, between the three wings, although still not to the same degree as the leading edge spars. The strain gage closest to the trailing edge of the wing had similar agreement as the previous strain gage. The results from the chord-wise group can be seen in Fig.( 5.11) below.

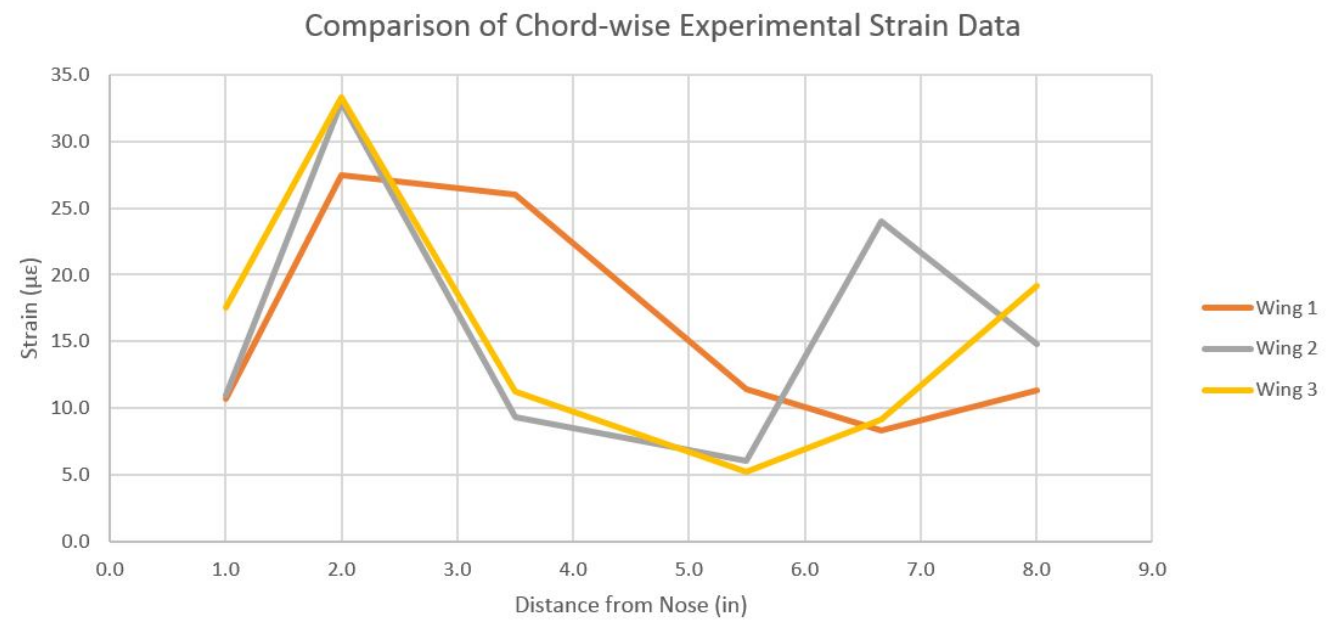

Figure 5.11: Strain values from the three wing models along the chord-wise direction 
These strain gages could also have been affected by torsion in the wing from the point load on top of the leading edge spar. Since the wing model is asymmetrical it would have needed the load to be applied to the center of mass to theoretically have zero angle of twist. Although for a wing in flight most of the lift force is concentrated at about $25 \%$ of the chord or on top of the leading edge spar. For this reason, it was decided to apply the point load at this location. No twist was visibly observed during testing so this is merely a possibility for what could have caused the results for this group to be erratic.

In order to have an idea of how much force was applied during the fatigue testing, a force and displacement study was done. This was important because the wing would not have a load cell connected to it to measure the force being applied.

After adding weights up to $8.3 \mathrm{lbf}$ and measuring vertical displacement, the data was imported into Excel so that plots could be made. This study was done with and without the front mount that is connected, to the Instron, to see if it would affect the data.

Similarly as other static testing, the test was repeated five times to insure that testing was consistent. After doing this for both instances, it was found that the wing displaced linearly for the load being applied. The displacement measurements, using the dial gage, were also very close to each other. The force and displacement curves with and without the mount can be seen in Fig.( 5.12) below. 


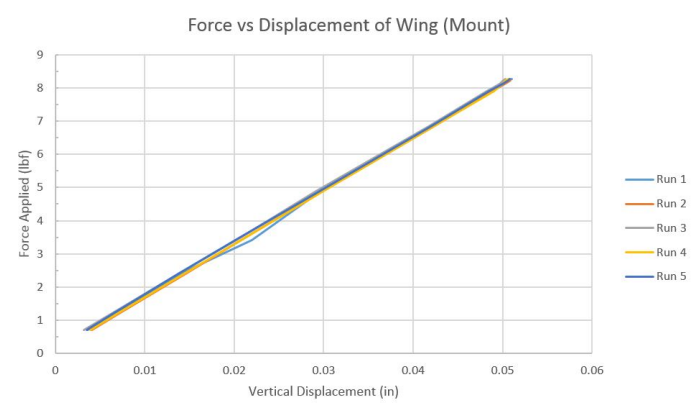

(a) Force applied vs displacement with the front mount

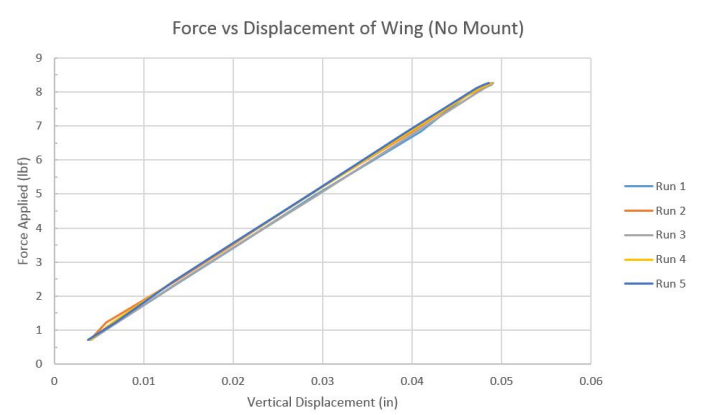

(b) Force applied vs displacement without the front mount

Figure 5.12: Displacement curves for the wing with and without the mount

Then the values from each configuration were averaged out so that a trend line could be made. The trend lines from the testing with and without the mount were then plotted on the same plot to be compared. It was found that up to $2 \mathrm{lbs}$, the two configurations displaced by about the same amount. At 2 lbs both test, had a displacement of around 0.011 inches which was a third of the displacement applied to the wing for the long term fatigue test. After this point, it was seen that with the mount the wing displaced more than without for the given load, although not by much. At the maximum load applied, the two displacements differed by $3.93 \%$ . The trend lines from the average data for each of test can be seen in Fig.( 5.13).

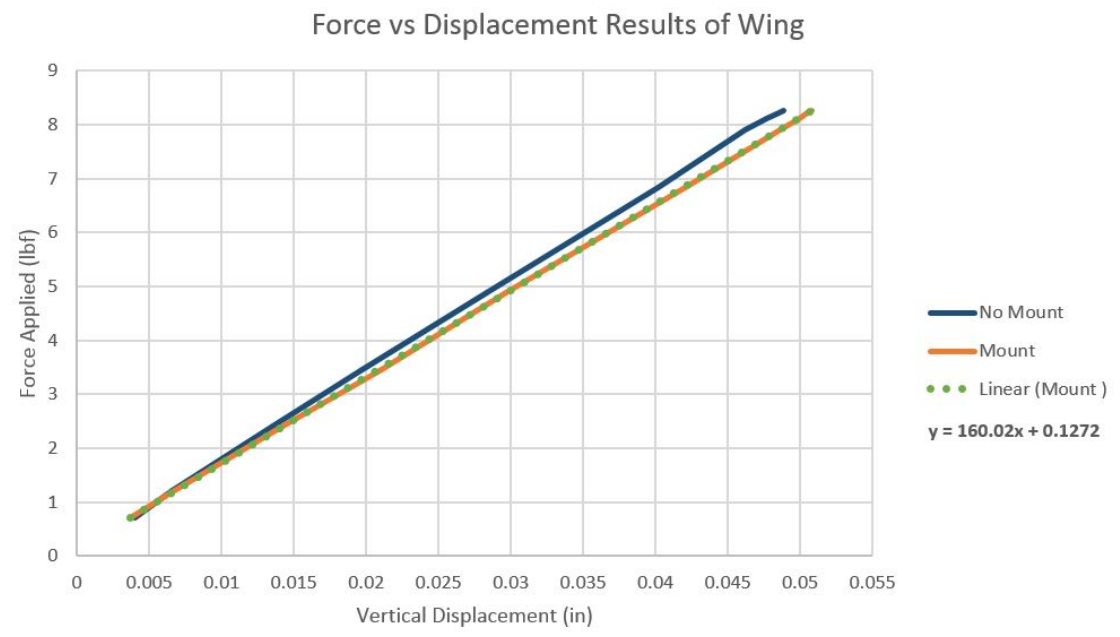

Figure 5.13: Comparison of the force and displacement study with and without the front mount 
From this curve it can be estimated that at the .03 in displacement amplitude, from the Instron, was applying a force of about $5 \mathrm{lbf}$. At the maximum displacement amplitude, that was tested in fatigue the force estimate was $8.25 \mathrm{lbf}$. These are just estimates and the only way to be certain of the load being applied would be to design a fixture so that a load cell could be used.

\subsection{Fatigue Test Results}

In this section, the results of the various fatigue testing studies are covered. The first subsection described the findings of changing the test frequency on the fatigue behavior. The next study was how the strain measurements compared between static and fatigue loadings. Lastly, the results of the fatigue life test of the wing models are described.

\subsubsection{Fatigue Behavior at Different Test Frequency Results}

The next study that was done came about due to the logistics of a fatigue test. The maximum number of cycles that the wing would be tested would be 100,000 which would take an unreasonable amount of time at a slow test frequency. A study was done to determine if the results would differ at different test frequencies

After testing the same wing model at $.01, .02$, and .03 in displacement amplitude, it was found the strain result at $1 \mathrm{~Hz}$ and $7 \mathrm{~Hz}$ differed by $2.0 \%, 7.3 \%$, and $8.5 \%$ respectively. All these differences were under $9 \%$ and since testing at $1 \mathrm{~Hz}$ would take longer than a day then $7 \mathrm{~Hz}$ would be a feasible test frequency. At $7 \mathrm{~Hz}$ the test would take almost 4 hours for each wing. Since there were 3 wing models to test this was a considerable amount of time. When tested at 10 $\mathrm{Hz}$ the strain values differed by $1.0 \%, 1.9 \%$, and .6 \% compared to $7 \mathrm{~Hz}$ for the respective displacements. By testing at $10 \mathrm{~Hz}$ a little over an hour can be saved per wing on test duration. The results for the 3 speeds and 3 displacements tested 
are tabulated in Table ( 5.8) along with differences in Table ( 5.9).

Table 5.8: Test frequency study results

\begin{tabular}{|c|c|c|c|}
\hline \multirow{2}{*}{$\begin{array}{c}\text { Strains } \\
(\mu \epsilon)\end{array}$} & \multicolumn{3}{|c|}{ Test Frequency $(\mathrm{Hz})$} \\
\hline Fatigue Amplitude (in) & 1 & 7 & 10 \\
\hline 0.01 & 48.6 & 49.6 & 49.1 \\
\hline 0.02 & 88.5 & 95.5 & 97.3 \\
\hline 0.03 & 128.5 & 140.5 & 141.4 \\
\hline
\end{tabular}

Table 5.9: Differences between test frequencies

\begin{tabular}{|c|c|c|c|}
\hline $\begin{array}{c}\text { Fatigue } \\
\begin{array}{c}\text { mplitude } \\
\text { (in) }\end{array}\end{array}$ & $\begin{array}{c}\text { Difference } \\
\text { between 1 and } \\
7 \mathrm{~Hz}(\%)\end{array}$ & $\begin{array}{c}\text { Difference } \\
\text { between } 7 \text { and } \\
10 \mathrm{~Hz}(\%)\end{array}$ & $\begin{array}{c}\text { Difference } \\
\text { between 1 and } \\
10 \mathrm{~Hz}(\%)\end{array}$ \\
\hline 0.01 & 2.0 & 1.1 & 1.1 \\
\hline 0.02 & 7.3 & 1.9 & 9.0 \\
\hline 0.03 & 8.5 & 0.6 & 9.1 \\
\hline
\end{tabular}

Since the differences in strain between the two faster speeds were small it was decided to test each wing at $10 \mathrm{~Hz}$ for the fatigue life test. If this test would have needed extremely accurate results where a deviation would be unacceptable then the wing would have been tested at $1 \mathrm{~Hz}$. Although to do this multiple operators would be needed to run the Instron machine in time segments. Precautions would also need to be taken to insure that the machine would not over heat which would damage it. In Fig.( 5.14) and Fig.( 5.15) the strain values for each test frequency at each displacement amplitude can be seen. These curves show how there is a difference between $1 \mathrm{~Hz}$ and $7 \mathrm{~Hz}$ but at faster frequencies the difference is small. 


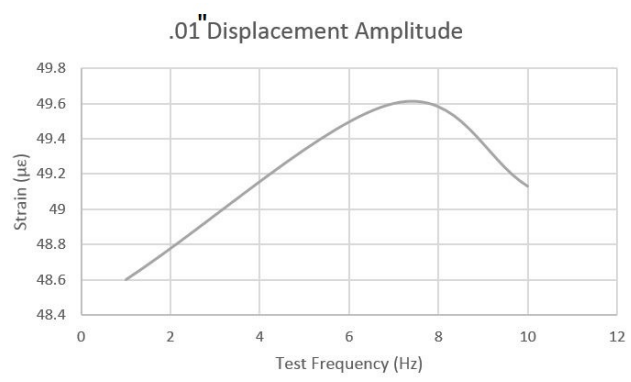

(a) Force applied vs displacement with the front mount

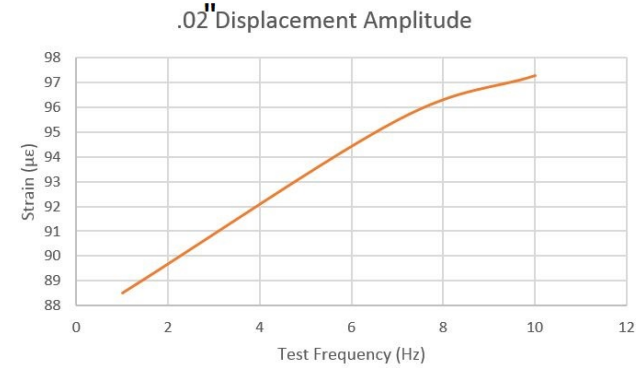

(b) Force applied vs displacement without the front mount

Figure 5.14: Displacement curves for the wing with and without the mount

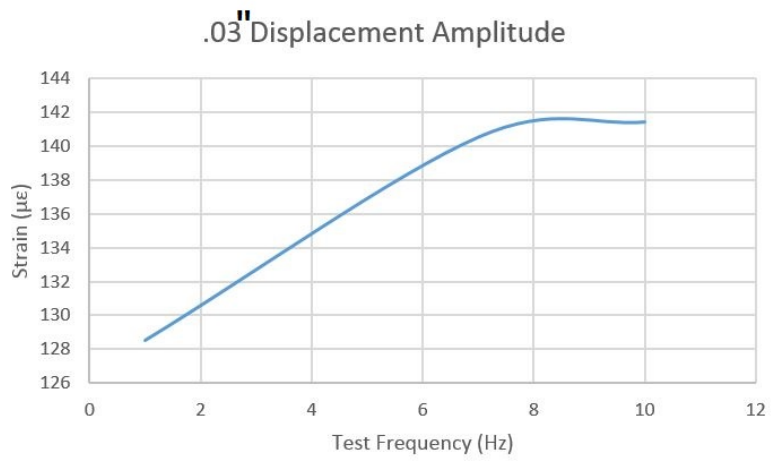

Figure 5.15: Comparison of the force and displacement study with and without the front mount

\subsubsection{Strain Behavior under Static and Fatigue Loading Results}

As mentioned before, the load could not be measured during the fatigue test, therefore methods were developed to estimate the load being applied. The second method used took advantage of the strain gage on top of the leading edge spar and closest to the fixed end. The wing models were tested at different fatigue displacements to see how the strains results compared to the static testing. Since the force applied during the static testing was known, a ratio could be used to estimate the load being applied during the fatigue testing. In order to limit the effects of the test frequency, the test was run at $1 \mathrm{~Hz}$. The ratio used can be seen in eq. ( 5.1) below. 


$$
F_{f}=F_{s} * \frac{\epsilon_{f}}{\epsilon_{s}}
$$

where $F_{f}$ is the calculated fatigue load in $l b f, F_{s}$ is the known static load in $l b f, \epsilon_{f}$ is the strain measured from fatigue testing in $\frac{i n}{i n}, \epsilon_{s}$ is the strain measured from static testing in $\frac{i n}{i n}$.

The known strains from the 2.41 lbf static load were $32.95 \mu \epsilon$ and $34 \mu \epsilon$ for wings two and three respectively. The ratio was used for strains from the fatigue testing at displacement amplitudes from .01 in to $.05 \mathrm{in}$. With this data a curve could be made estimating the force due to different displacements put on the wing model. The results are shown in Fig.( 5.16) along with the force and displacement study results.

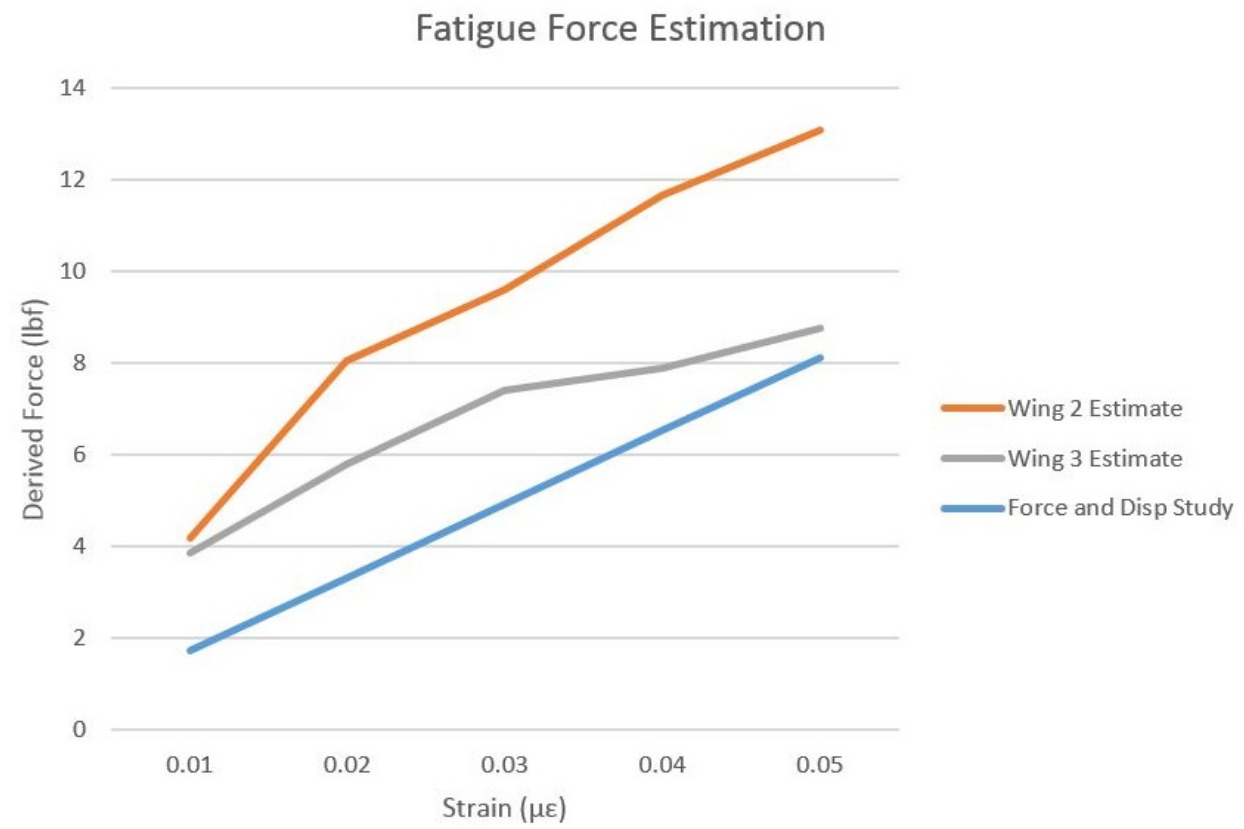

Figure 5.16: Fatigue force derivation from static and fatigue stain comparison

It can be seen that using this method to estimate the force applied did not yield a trustworthy trend. It would be expected for a cantilever beam structure to have a linear relationship between force and displacement. Wing 3 followed a 
relationship that was closer to linear than the second. Although except for the .01 in displacement data there was a considerable difference between the results from the two wings. The force and displacement study yielded a trend that provided more confidence in force estimations. Therefore it was concluded that the strains could not be compared between the two different test types in order to estimate the force being applied to the wing model.

Only the results from the second and third wings were shown, since there was a faulty strain gage on the 1st specimen. This strain gage could have been damaged by the fatigue displacement by debonding or a broken wire connection. No physical damage could be seen but strain readings were found to be low based on previous testing.

\subsubsection{Fatigue Life Test Results}

During the long term fatigue test, the wing was observed to insure there was no damage propagating on the wing. The mount and fixture bolts were also checked periodically to insure that they had not loosened from the vibration. Since lock washers were used with the bolts, they never had to be re-tightened.

Below in Fig.( 5.17), the strain measurements from the strain gage on the leading and trailing edge during the test can be seen. Following the same trend, as the static test, the strain at the leading edge was higher than the trailing edge. This snapshot of the test is for 1 second and it can clearly be seen that 10 cycles were completed in this amount of time. This also gives assurance that the wing displaced vertically in the upward direction and then in the downward. There is a slight difference in strain between the upward and downward displacements which could have been error in mounting. It also could have been due to inconsistent bond areas between the top and bottom of the wing. 


\section{Strain Measurement during Fatigue Test}

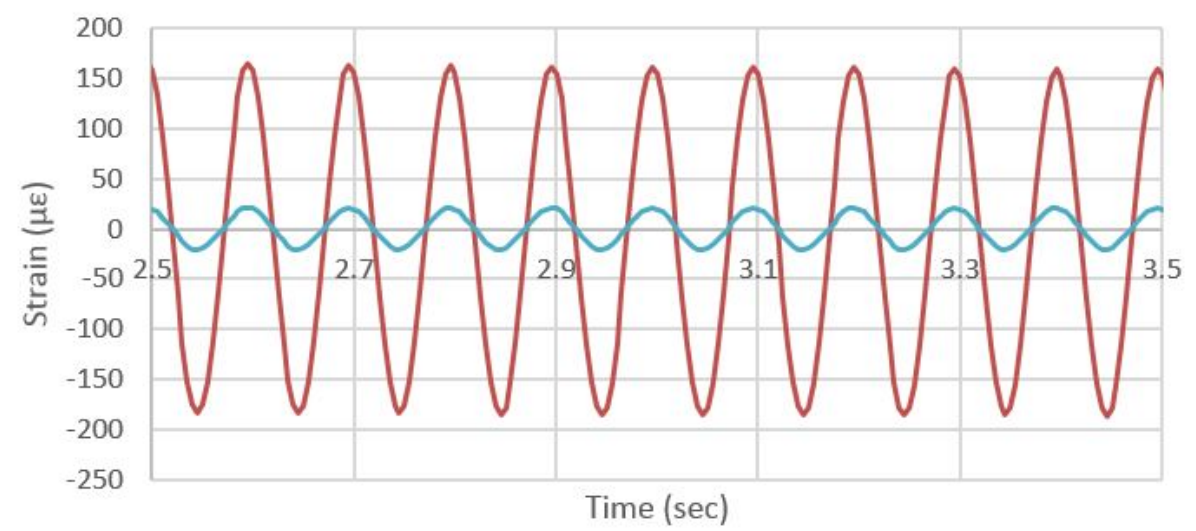

—Leading Edge Gage —Trailing Edge Gage

Figure 5.17: Stain measurements during fatigue test

By observation, the wing models were being displaced by a significant amount since the strains were higher than the static testing. The vertical displacement of the wing was easily seen since the maximum vertical displacement was .06 in , twice that of the cyclic amplitude displacement. The wing models were rather stiff so it was interesting to see that they deflected to these amounts repeatedly. The fixture did experience minimal vibrations although it did not seem to affect the strain results being measured by DAQ.

None of the wings suffered visual damage after 100,000 cycles had elapsed. At this point each wing was removed since it was expected that it would not break due to this displacement amplitude. This was surprising since it was expected for the composite material to withstand the repeated strains, but the bonded joints to fracture first. A possible reason that the bonds did not break was that the adhesive did not cure well, causing it to have flexible characteristics. Although throughout the assembly process, it was seen that the adhesive cured well due to careful mixing of the resin and epoxy. Due to the diligence, when preparing the adhesive, one would not attribute the survival of the wing due to the epoxy. Another reason that the wing did not fail was due to the wing being too stiff for the loading. A solution 
would be to reduce the amount of layers of composite throughout the wing model. The cyclic displacement could have also been increased to cause a failure.

Since only three wings were available for data collection, it would have been difficult to find a displacement that would allow the model to be fatigued and not break at the first cycle. When fatigue testing is done, in industry, there are numerous of samples that are made so that a fatigue test can be developed. Although when the models are expensive to manufacture, when its comes to hours and cost, proto-flight testing is ideal. Proto-flight testing involves testing to be done on the model that would also be used for the final product. The proto-flight model would have loads higher than expected in its mission to be imparted on it. These types of programs are risky since a full qualification of its abilities is not tested. Proto-flight testing should be reserved when material and time cost are substantial to manufacture the models.[34]

Strain data was collected throughout the entire fatigue test although it was found that the values gradually offset over the hours of the test. This could have been caused due to loose connections, since the wires were just twisted to each other. Another possibility could have been that a change in ambient temperature could have affected the measurements or possibly electrical interferences. The solution to the ambient temperature issue would have been to use temperature compensating strain gages. These strain gages would be put on an unloaded specimen of the same material to cancel out the strains due to temperature. Although this was not done because it is essential to keep both the loaded and unloaded strain gages at the same exact temperatures. Since it was not possible to do this it was decided to carry on without them. This offset of the strain measurement, only affected the long term test since the time frame was hours. The static test at most lasted 10 seconds where the ambient temperature change in the room was negligible. To eliminate electrical interferences from the measurements, all devices 
emitting these signals would need to be turned off throughout the test. An example a device that could send out electrical interferences would be an electric motor. One way around locating electrical interferences and turning them off, would be to use wires enclosed in electrical shielding. Wire with this type of shielding is more expensive than traditional wire. Carrying out the prior two solutions would have not made it certain that drift would not have occurred, since the cause is unknown.[35]

\subsection{Static Test after Fatigue Test Results}

After the fatigue tests, the wing models were tested statically once again to insure that no damage had occurred from the fatigue testing. When the testing was finished, the data from the leading edge strain gages from each wing were compiled in an Excel spreadsheet. Only data from the leading edge strain gages was taken since this was by far the most consistent data.

The percent differences between the initial and post-fatigue static test for the three wing models were $4.2 \%, 4.8 \%, 3.0 \%$. All of these differences were small. These deviations could have been caused from testing at different conditions including, mounting differently, and variability in loading, and environmental. The difference for each strain gage along the length of each model can be seen in the plots in Fig. ( 5.18) and Fig.( 5.19) below. The plots show how little the difference is between the two test results for each strain gage. If damage had been done to the wing model there would have been a considerable difference between the results. If the wings had been damaged then the strains would have been far lower than the initial test. The stains would have decreased because damage would have lowered the stiffness of the wing, allowing it to displace more without resisting the force. A difference of $10 \%$ would have been closer to being considered damage, and a

difference of $20 \%$ would have been classified as damage to the structure. Since 
the percent differences were far below both of these thresholds, it was determined that there was no damage to the specimens. This study is in agreement with the visual observations which tell that no damage occurred from the long term fatigue tests.

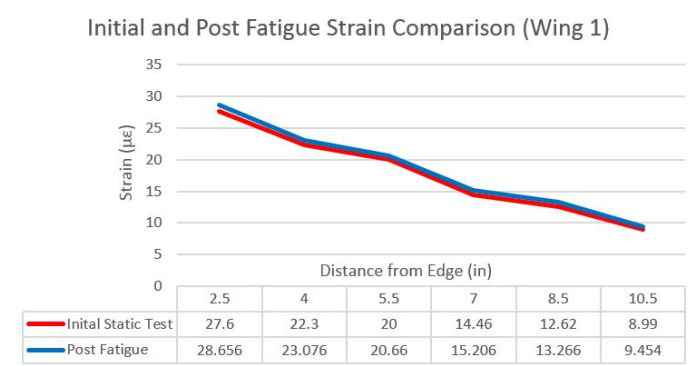

(a) Percent difference between initial and post fatigue testing of wing 1

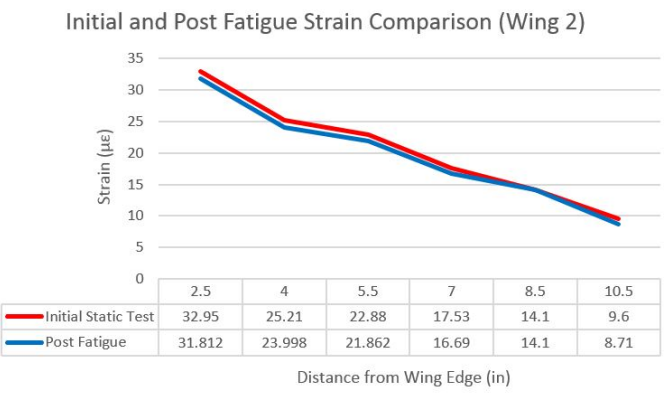

—Initial Static Test —Post Fatigue

(b) Percent difference between initial and post fatigue testing of wing 2

Figure 5.18: Displacement curves for the wing with and without the mount

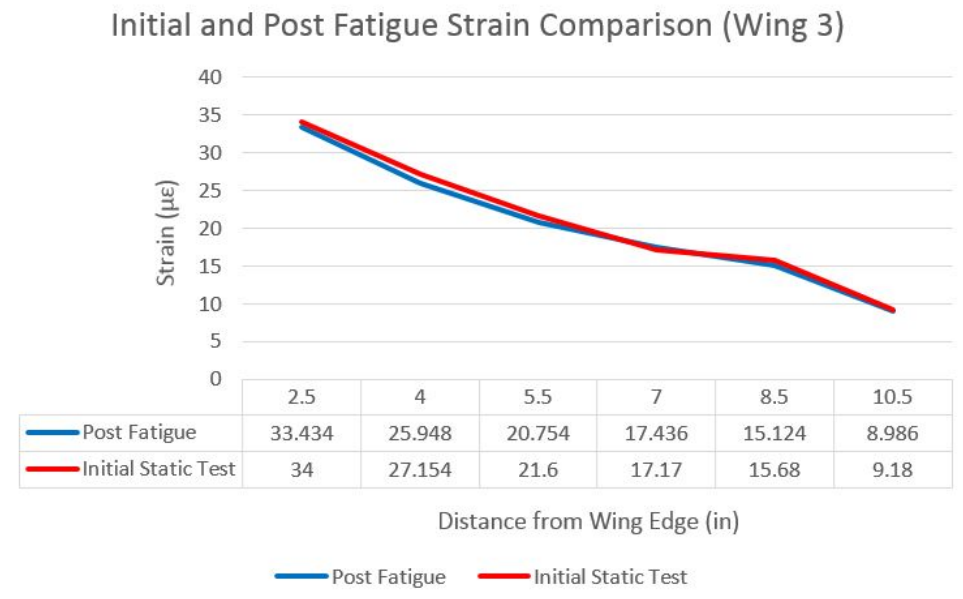

Figure 5.19: Percent difference between initial and post fatigue testing of wing 3 
Chapter 6

FEA SETUP AND RESULT DISCUSSION

This chapter covers the FEA software utilized and how the results were validated. After validation, a review of the wing model is discussed, and then the procedure to imported into ANSYS is explained. To insure that the FEA model is trustworthy, a mesh convergence was also performed and discussed in this previous section. Lastly the static and fatigue results using the FEA model are outlined and discussed.

\subsection{Selecting an FEA Solver}

The initial FEA software that was utilized for this thesis was Abaqus CAE 6.14 due to availability and familiarity. The static analysis were straight forward, and simulations were run for different types of loads to insure that the results were reasonable. Although when it came time do preliminary fatigue simulations, it proved to be complicated and little resources were available. Some resources even mentioned that a software extension would need to be installed to do fatigue solutions. This external software such as fe-safe was not available and would not be within the budget of the project.[36]

After much research it was found that Abaqus used an extrapolation method for cyclic loads. The solver would run a short amount of cycles, and then based on Hashin damage criteria it would determine if damage occurred, and how it evolves. Then it would calculate the same amount of cycles again, and if damage was damage present it would determine how the damage would propagate for 
every cycle. This method is very efficient computationally since it solves the whole fatigue test iteratively. Variables that are used in its damage initiation and evolution equations must be tested for, and it was not possible in our lab. These variables required energy measurements in small increments of time to be able to model cracks in a composite laminate. Due to this complexity other options were explored.

Another FEA software that was available was ANSYS 16.0, which I had not used extensively. Its interface proved to be very user friendly, and operated similarly as to Abaqus when running static analysis. A video by CAE Associates Inc. was found that showed detail steps on how to define a composite layup for a part. This proved very useful so that the structures could be correctly modeled as a laminate. Then it was researched what material properties ANSYS would require in order to run a fatigue analysis. ANSYS software also had a fatigue tool that made fatigue analysis simple to setup. This tool allowed the user to easily choose fatigue options and loads for the analysis. When a material in the ANSYS database was chosen it was seen that it had an S-N curve in its properties window. What this curve shows is how many cycles the material would withstand, at a certain percentage of its ultimate strength. When the fatigue analysis was run for a piece of aluminum it finished successfully, then offered damage, and life results. Due to the ease of use that ANSYS provided, it was determined to continue with it as the primary solver in this project.[37]

\subsection{Model Validation FEA Results}

The simplest case analyzed was an aluminum cantilever beam that was loaded 1 inch away from its free end. This study was done to insure that our methods used in the FEA model are correctly modeling a cantilever structure and its boundary conditions. For the simulation a solid model was made using the dimensions from 
the experimental test, as well as the boundary conditions and loads. It was meshed using SOLID 186 elements with an edge size of $0.05 "$ ". The reason that a shell model was not used is because the beam thickness was greater than a quarter of an inch. Additionally when using solid elements the detailed stresses in the structure could be investigated. Since not many solid elements were used for the aluminum beam computation time was not a concern.

The ACP Pre software used to model the composite plies in the Cycom and Hexcel beams required that shell elements be used. Shell elements are recommended for composite structures because of their thin features. Inaccuracies could also arise from using a solid element for a thin part. The FEA results of the composite beams will be discussed after the aluminum beam results.

After running the simulation for the aluminum beam the results were exported for post processing in Excel. The FEA model showed the correct strain distribution where it would be highest at the fixed end, and would diminish at the free end. This distribution can be seen in Fig. (6.1) where the highest strain at the root was about $200 \mu \epsilon$.

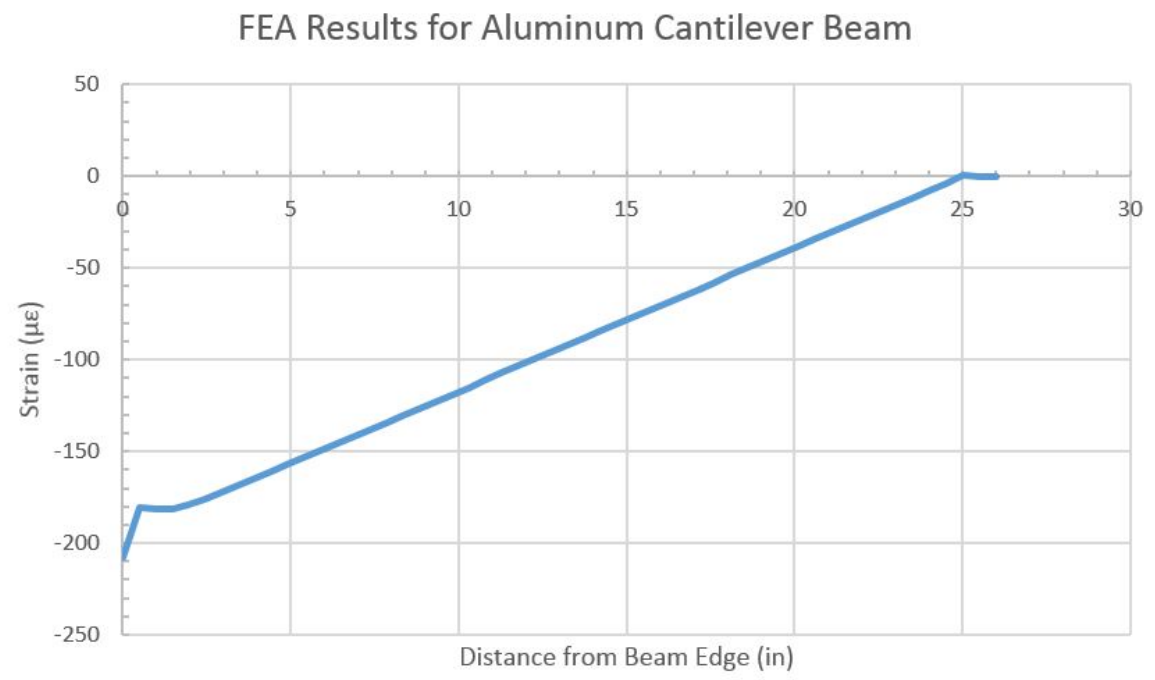

Figure 6.1: Strain distribution along the aluminum cantilever beam from FEA model 
The second study that was simulated with an FEA model were cantilever beams for each of the composite materials. A shell model of the beam with dimensions of 1" by 11" was made in ANSYS and given a thickness of $0.115 "$. Then the material properties tested for were inputted into the software. After this was done ACP Pre was used to define the composite materials as a fabric, and the number of layers that were used in the part were assigned. Care was taken to align the orientation of the composite as it was in the experimental test. Then the beam was constrained in the same manner as the experimental test. A square inch on one side was held fix, and at the tip a load of $1.302 \mathrm{lbf}$ was added. The boundary condition and force locations can be seen in Fig.( 6.2).

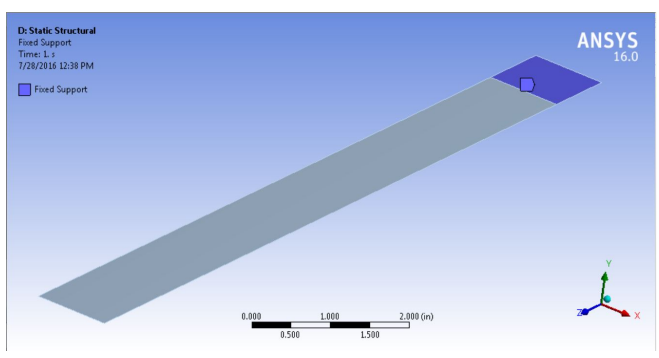

(a) Fixed constraint on the beam shell model

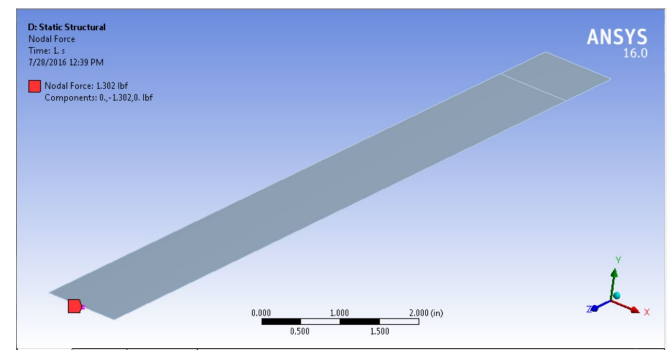

(b) Load location on the beam shell model

Figure 6.2: Cantilever beam model setup in ANSYS software

Next an element size of 0.05" was chosen, and meshed using SHELL 181 elements. This yielded 4620 elements on the part. Since the strain gages were placed along the center line of the beam, a path was made on the corresponding nodes on the model. This will allow a curve of the strains along the beam to easily be displayed. Since nodes were not located at the exact locations where the strain gages were placed, an interpolation method was used to obtain the result for the desired location. This was possible since the trend of that data was linear. In Fig. ( 6.3) the mesh that was used, as well as the resulting strains on the path can be seen. 


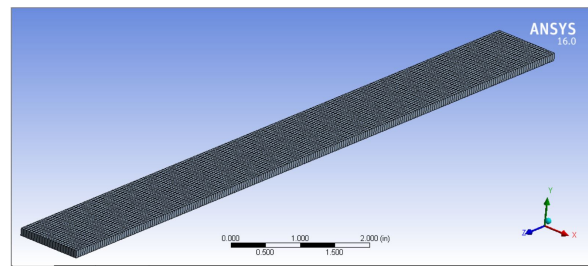

(a) Meshed cantilever beam model in ANSYS software

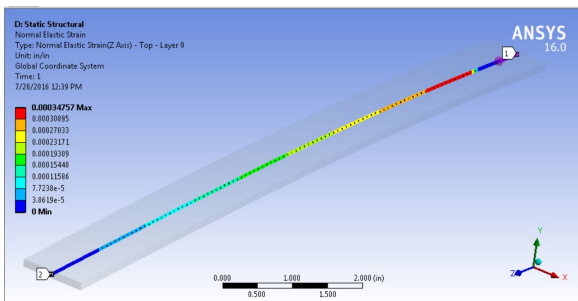

(b) Strain in the $\mathrm{Z}$ direction results along the path

Figure 6.3: Cantilever beam mesh and strain results

Results from each composite beam followed an expected trend for a beam fixed at one end, and loaded at the tip. The displacement curves showed a cubic relationship, which is expected for a cantilever beam. The displacement curves for each material can be seen in Fig.( 6.4 ). The two beams were loaded by the same force, although the Cycom beam displaced more because of its lower modulus.

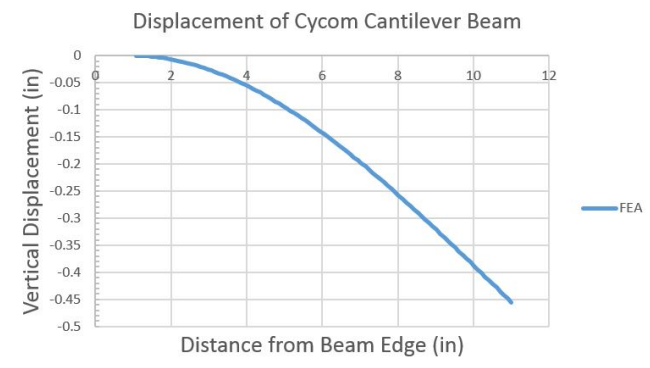

(a) FEA displacement curve for the Cycom material

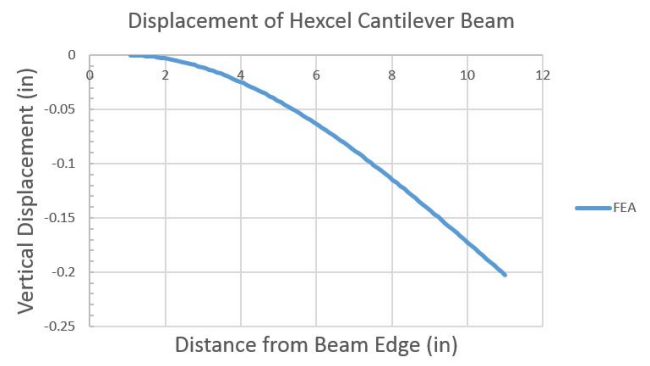

(b) FEA displacement curve for the Hexcel material

Figure 6.4: Strains along the cantilever beams for both materials used in wing model

The strains were highest at the fixed end, and diminish to zero at the free end. Since the structure is free to move at the load location, it does not carry any load, therefore had small strains. It can be seen that the Cycom beam measured higher strains than the Hexcel beam, even though the loads were identical. This is because of the lower stiffness of the Cycom material found through material testing. The curves for each beam also show a linear representation, which is expected for this type of loading and structure. Both strain distributions can be 
seen in Fig. ( 6.5) below.

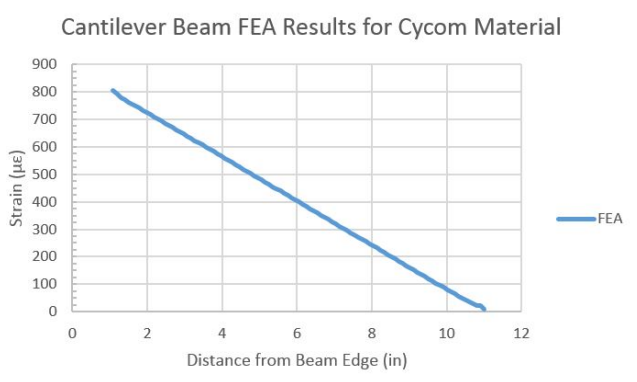

(a) FEA strain curve for the Cycom material

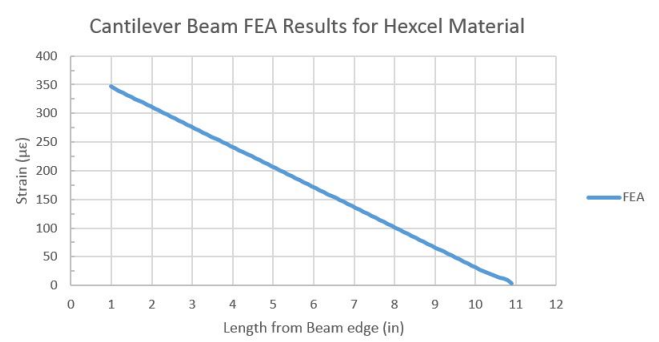

(b) FEA strain curve for the Hexcel material

Figure 6.5: Strains along the cantilever beams for both materials used in wing model

\subsection{Building the Wing Model}

In order to build the wing model in SolidWorks, the airfoil points would need to be obtained. This was done by choosing the NACA 0016 airfoil in the XFLR software, and exporting the points for a chord length of 1 inch. Then these points were imported into the SolidWorks CAD software. A spline tool was used to make a continuous curve through the airfoil points. Since the actual chord length of the wing was not $1 "$, the airfoil curve was scaled out to the desired chord dimensions. Then this curve was extruded to the length of the wing as a thin shell surface. This would be the part that would model the wing skin.

After this the spar locations were located along the chord of the wing skin and the cross-section of them was modeled. Then the face of each spar was extruded to the length along the wing, so that each side would be flush with the rib parts. The spars were also modeled as shell elements to maintain consistency in the model. The last part to be included in the assembly was the ribs which were made by taking the airfoil shape, and making a surface out of it. Parts with thickness were positioned offset from each other, so that after the thickness is included it they would be flush. The finished shell model in SolidWorks can be seen in Fig.( 6.6) 
below.

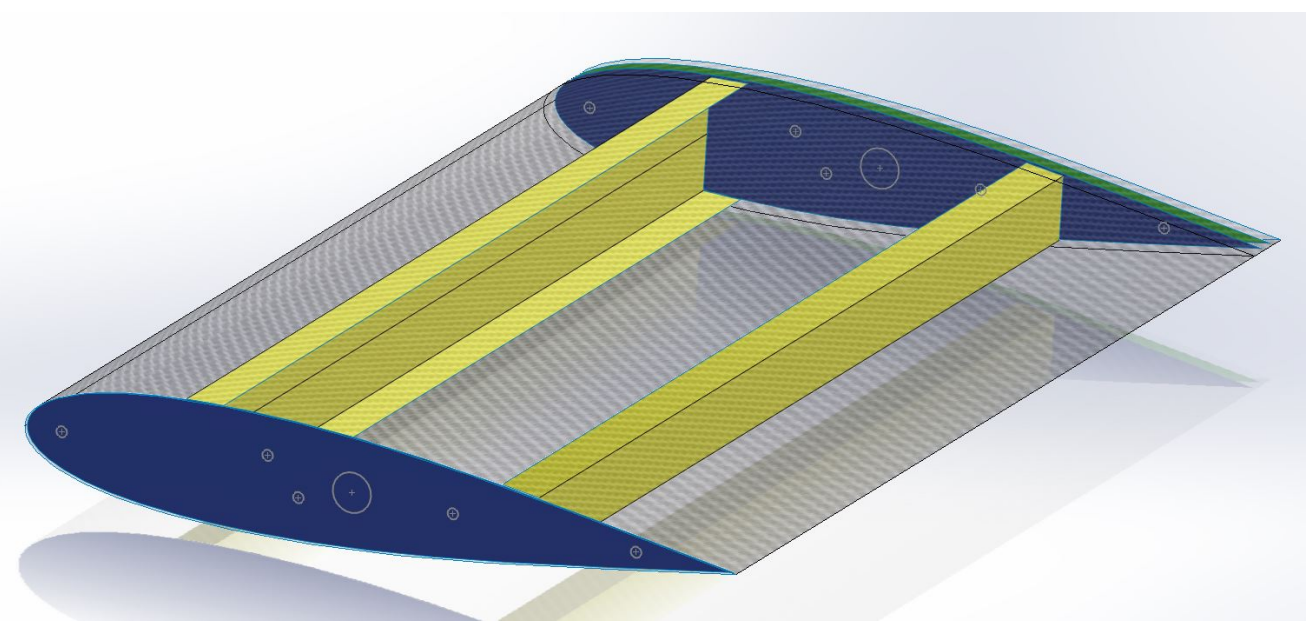

Figure 6.6: Shell model made in SolidWorks ready to be imported into ANSYS software

Now that the wing was modeled as a 3D shell assembly, it was then imported into the ANSYS FEA software using an ACIS (.sat) file. Many different types of file types can be used, but ACIS was used throughout the project. The wing model used the same two materials as the cantilever beams, therefore the same material properties were used. In order to define the composite layups in the wing model, ACP Pre was used in the same manner as the cantilever beams. Each part was assigned a set number of composite plies to represent the layup, as well as the fabric orientations.

After this the face of the root rib was selected to be fixed, to model the attachment to the fixture. In order to model the clamping areas from the fixture, these areas on the top and bottom were defined as fixed as well. Since the load in the static testing was a concentrated point, the load in the FEA model was assigned to one node at the end of the leading edge spar. The boundary conditions and loads can be seen in Fig.( 6.7). 


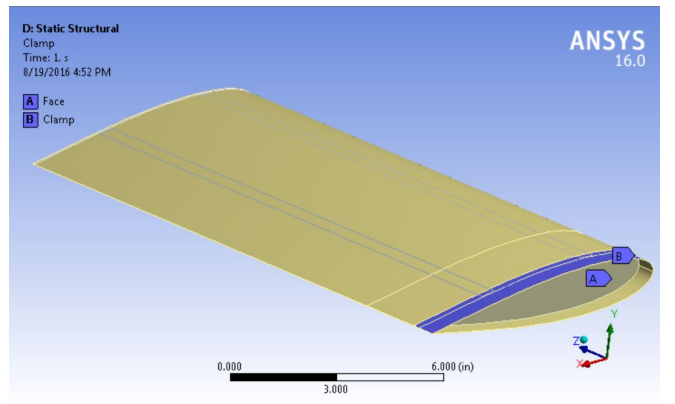

(a) Boundary conditions on the wing to model the experimental test

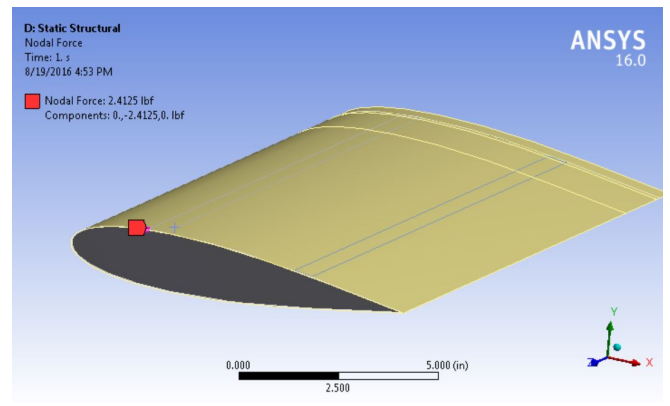

(b) Point load on the wing model to model experimental test

Figure 6.7: Setup of FEA wing model in ANSYS software

Then the wing model was meshed using the same SHELL 181 elements, used in the cantilever beam simulation model. In order to insure the mesh density did not affect the results, a convergence study was performed. The point of comparison between the models was 2.5 inches away from the root edge of the wing along the leading edge. This was the location of the strain gage closest to the fixed side, which would measure a higher strain compared to the rest. The average strain from the three wings at this location was $31.5 \mu \epsilon$ and would be compared to the FEA models.

The element size was changed from $0.75^{\prime \prime}$ to $0.125^{\prime \prime}$. For each mesh size the model was simulated, and the result from the strain gage was recorded, and compared to the experimental value. In Table ( 6.1) all the element sizes and their respective node and element counts for each model were shown. It also shows the difference in the strain value between the FEA and experimental data for each mesh density. The smaller the element used, the greater accuracy in the model due to it capturing the stress at a smaller area on the model. 
Table 6.1: Summary of the mesh densities used in the mesh convergence study

\begin{tabular}{|c|c|c|c|c|}
\hline $\begin{array}{c}\text { Edge size } \\
\text { (in) }\end{array}$ & Nodes & Elements & $\begin{array}{c}\text { FEA Result at } \\
\text { Gage }(\mu \epsilon)\end{array}$ & $\begin{array}{c}\text { Percent Difference } \\
\text { between FEA } \\
\text { and EXP (\%) }\end{array}$ \\
\hline 0.125 & 24432 & 24052 & 30.3 & 3.9 \\
\hline 0.15625 & 15775 & 15475 & 30.7 & 2.6 \\
\hline 0.1875 & 11058 & 10808 & 29.4 & 6.7 \\
\hline 0.25 & 6059 & 5946 & 28.8 & 8.6 \\
\hline 0.375 & 2746 & 2672 & 26.6 & 15.6 \\
\hline 0.4375 & 2040 & 1978 & 20.6 & 34.6 \\
\hline 0.5 & 1563 & 1509 & 18.5 & 41.3 \\
\hline 0.75 & 738 & 695 & 6.11 & 80.6 \\
\hline
\end{tabular}

After compiling all the data from the mesh convergence study, the strain result from the leading edge from each model was plotted against the number of elements in the model. This plot shows that by increasing the number of elements, which reduces the mesh size, would make the results converge to a value. The consequence of increasing the number of elements for the sake of accuracy, is longer computation times. It can be seen in Fig.( 6.8) that the plot converges to a value of about 30 $\mu \epsilon$, which is very close to the experimental data. This trend could also be seen by the diminishing error in the previous table above.

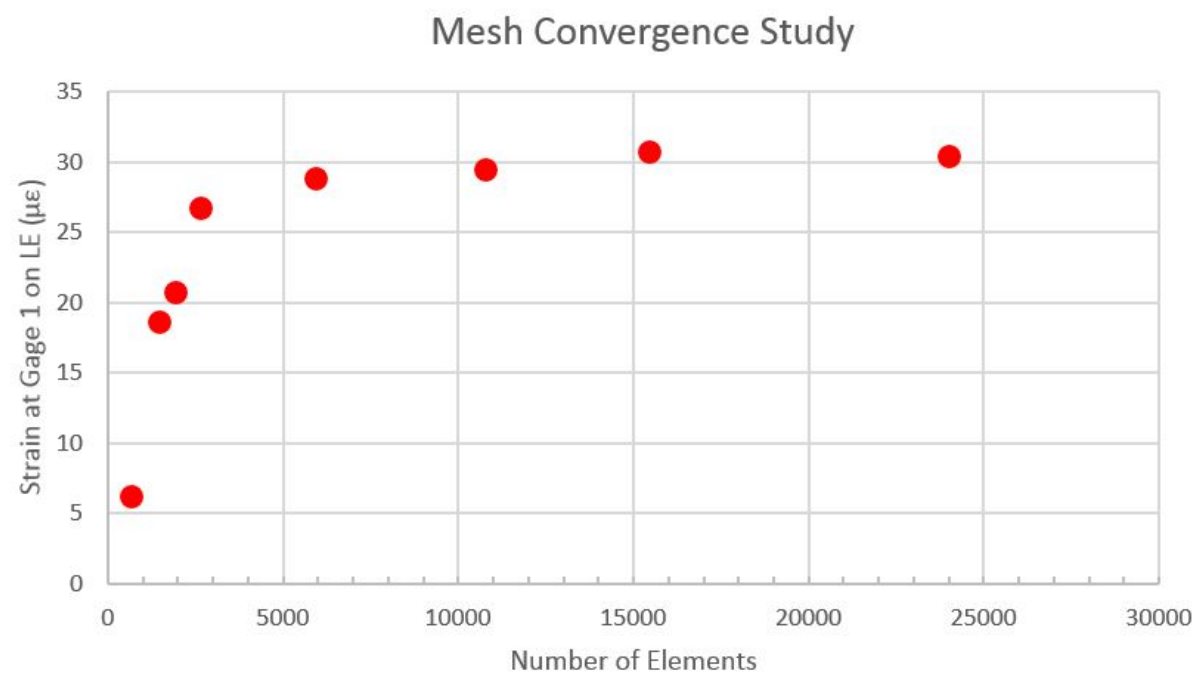

Figure 6.8: Convergence of strain result due to mesh density 
The final element size that was chosen was $0.15625 "$ since this provided the least amount of error, and did not take as long to complete than the $0.125 "$ mesh size. A comparison of the coarse mesh and the final mesh can be seen in Fig.( 6.9). The final mesh size chosen only took about 4 minutes to complete, which allowed for multiple models to be simulated without extensive delay. If solid elements were used, the computation times for each mesh size would have been considerably longer. Solid elements also could have lead to inaccuracies since the composite parts being modeled were very thin. It also not advised for thin parts to be modeled as solids, and in this case the ACP Pre software only allowed for shell elements to be used.

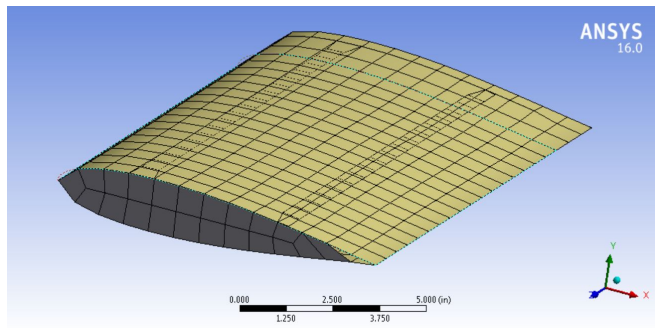

(a) Course mesh with 0.75" element size on the wing model

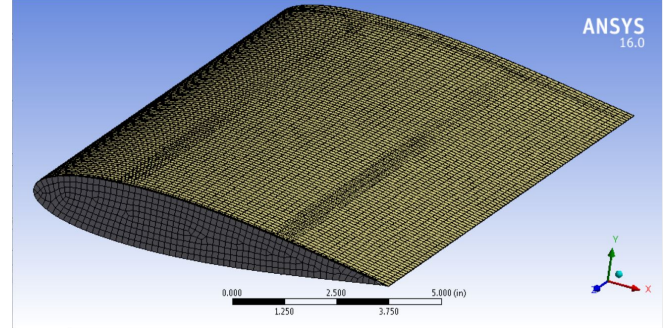

(b) Final mesh used for the wing simulations

Figure 6.9: Comparison of mesh sizes used in the convergence study

After the mesh size was decided, paths were made along the leading and trailing edge spars, so that the strain results could easily be viewed at these locations. A third path was also placed 2.5" away from the root edge so that the chord-wise strain results could also be viewed easily.

\subsection{Static Simulation Results}

Three areas were the main interest points when looking at results from the FEA simulation. These included the leading edge spar, trailing edge spar, and $2.5 "$ from the root edge in the chord-wise direction. These three areas had strain gages bonded to them for the experimental testing. 
The first strain gage group that was looked at was the strain gages on top of the leading edge spar. It was seen that the FEA results showed a trend expected from a cantilever structure, which was similar to the experimental testing. This trend can be seen in Fig.( 6.10) where the strain along the wing model is plotted. The strain was highest near the fixed condition, and lower in magnitude further away from this point. It was also observed that the strain was zero where the wing was being clamped by the metal fixture parts. By fixing this area with the fixture the stress was reduced at the spar and rib joint location. The solution for reducing the stress at the root joint is reinforced by the FEA results, since the strains in this area in the model were low. The path from where the leading edge strain results were probed from is shown in Fig.( 6.11).

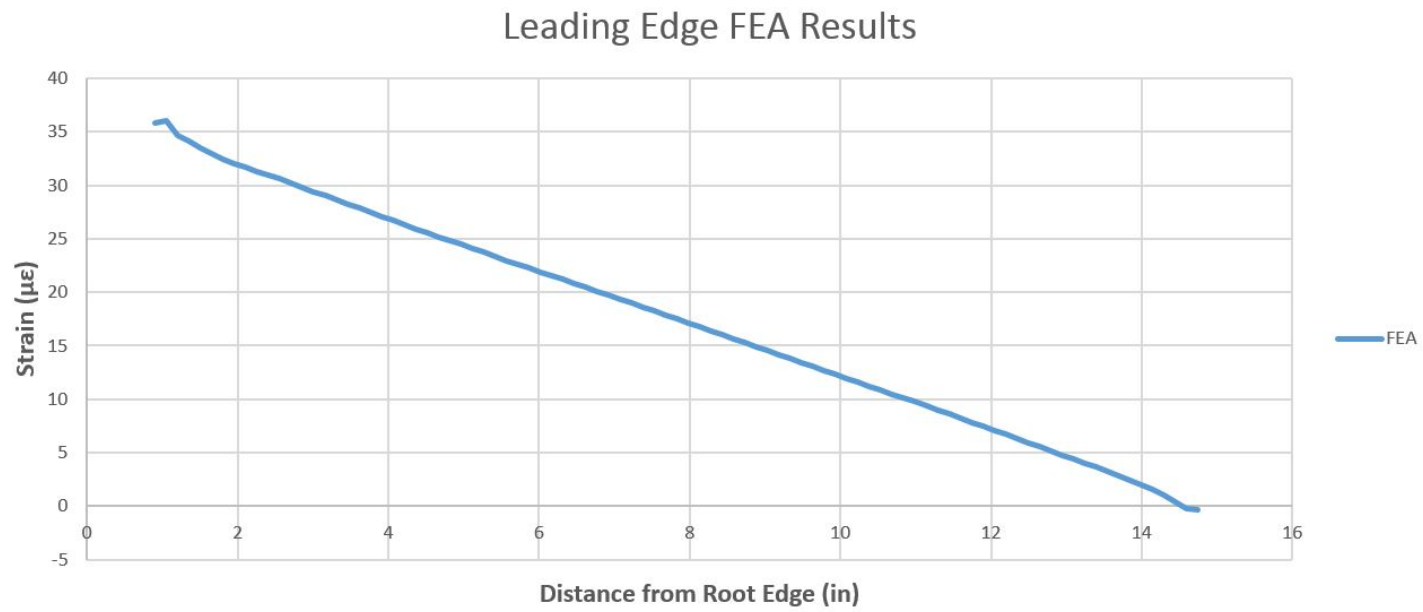

Figure 6.10: Strain distribution along the leading edge spar from the FEA simulation 


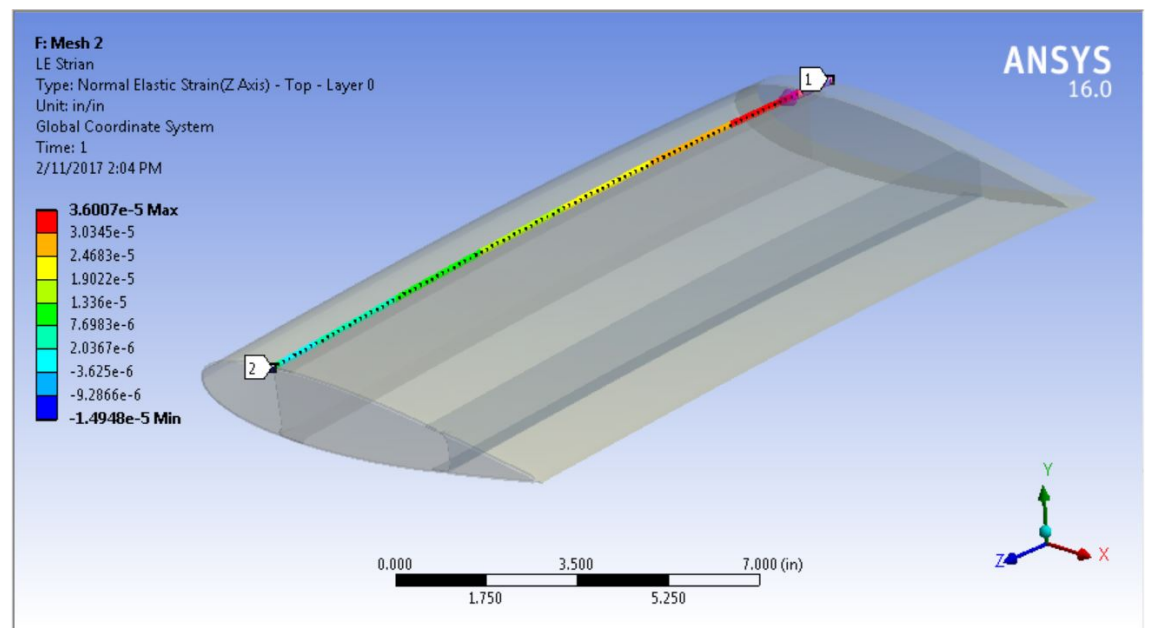

Figure 6.11: Strain result path for the leading edge spar results

The next group of interest was the trailing edge spar, which had its strain gages at the same locations span wise than the leading edge group. The curve for this area was very similar to the leading edge showing a diminishing strain from the fixed to free end. The strain distribution trend can be seen in Fig.( 6.12), where the strain vs the distance along the wing was shown. The strains overall were lower, which was expected since the load was centered at the leading edge. This was expected and for this reason the trailing edge spar was made thinner than the leading edge. Just like the last group discussed there was very low strains at the clamped area where the wing was held fixed. For the most part the distribution followed a linear trend expected in a cantilever structure. The path used to probe the strain results from the trailing edge is shown in Fig.( 6.13). 


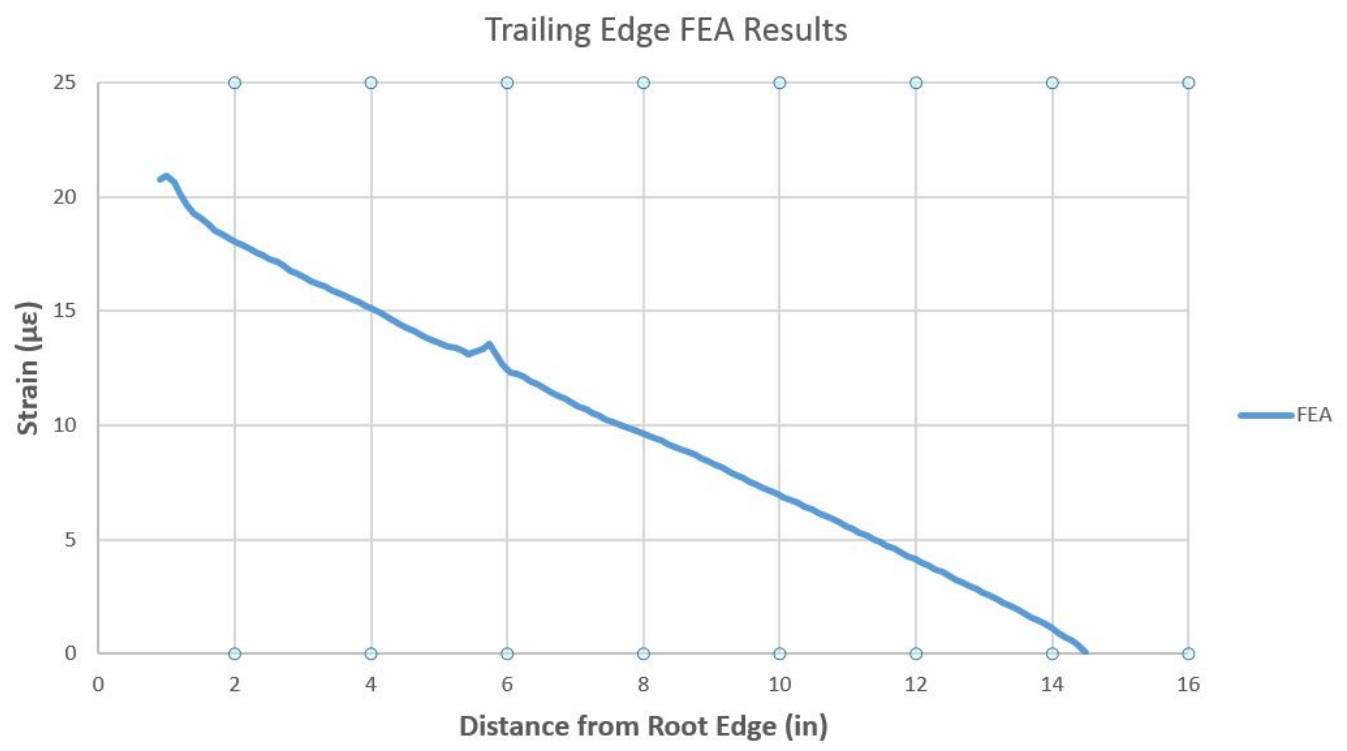

Figure 6.12: Strain distribution along the trailing edge spar for FEA and experimental data

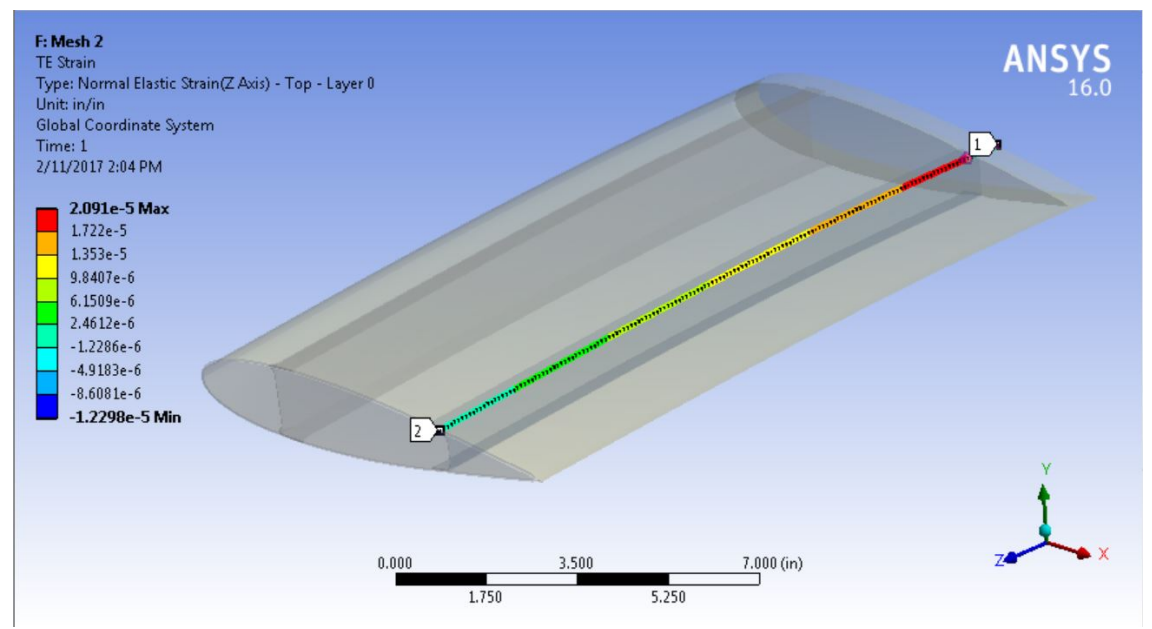

Figure 6.13: Strain result path for the trailing edge spar results

The FEA results for the chord-wise group showed a reasonable strain distribution that has an inverted parabolic shape that starts at the nose. This curve can be seen in Fig.( 6.14), where the strain along the chord at distance of 2.5 inches from the fixed end are plotted. The FEA results show that the strain increases sharply from the nose of the model, till the leading edge location. Thereafter the strain decreases until the trailing edge. This agreed with our design influences to 
make the leading edge thicker than the trailing edge, since more stress would be carried through this location. The strains peak at a value of around $32 \mu \epsilon$ just inside of the leading edge where the skin is solely taking the load. This would lead me to believe that in the simulation, the skin is carrying a significant portion of the load. The path from where the chord-wise results were probed from are shown in Fig. ( 6.15).

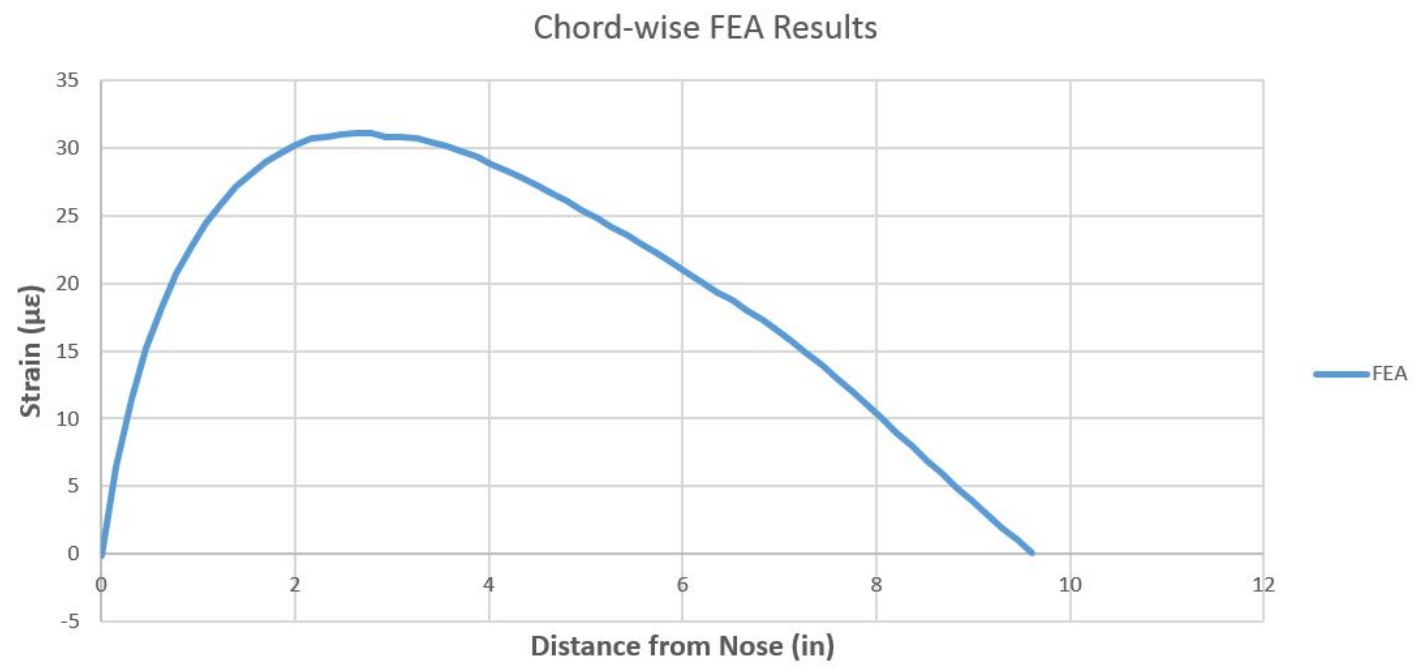

Figure 6.14: Strain distribution in the chord-wise direction for FEA and experimental data

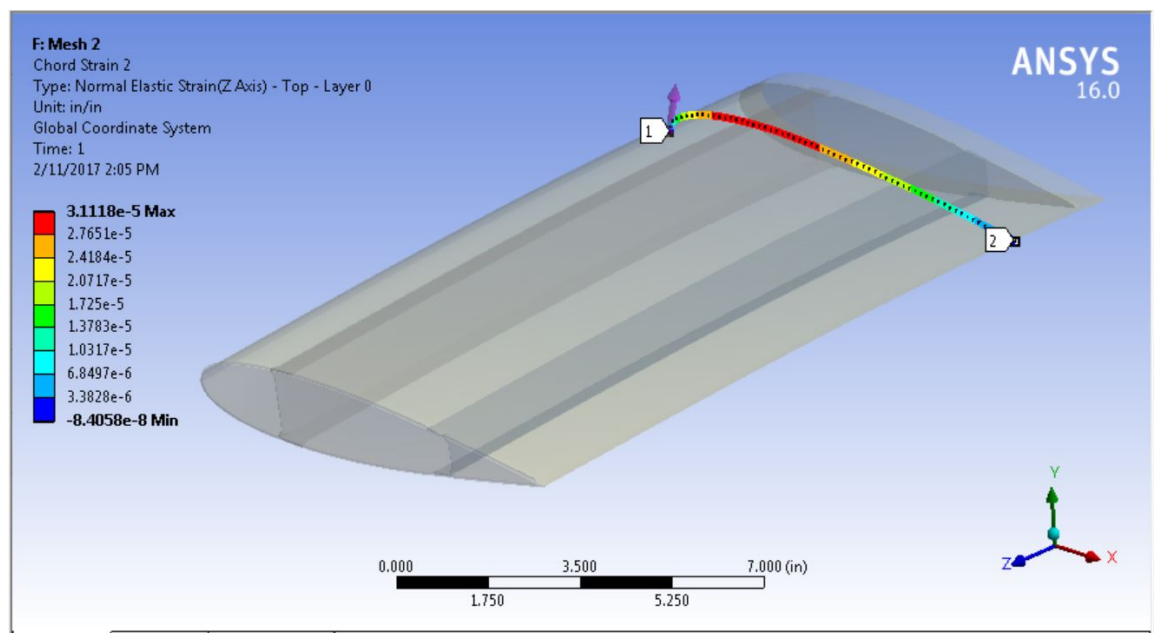

Figure 6.15: Strain result path for the chord-wise results

Five total spar geometries were compared to investigate which was optimum 
at resisting a force at the wing tip. Besides the $\mathrm{C}$ - channel the other designs included Z - spars, I beam, Box spar, and a box with flanges. The surface area of each spar design was held constant by keeping the flanges the same length, and not adding any additional thickness to the web. In the case of the box beam, half of the thickness of the I beam flange was used since there were two vertical webs. By doing this it would insure that the extra stiffness of the design was not due to increased material or thickness. The additional spar designs, not including the Cchannel can be seen in Fig.( 6.16) and Fig.( 6.17).

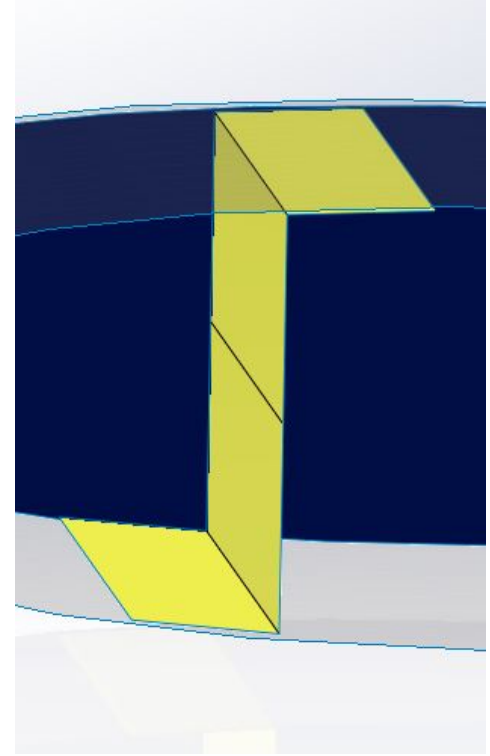

(a) Z - spar design

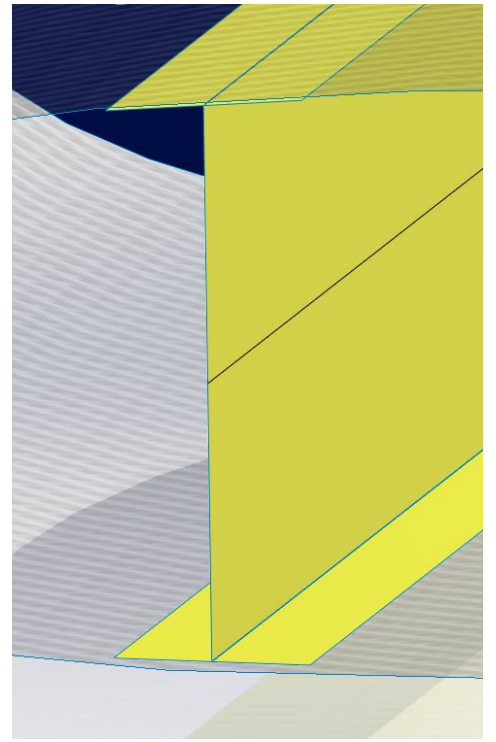

(b) I beam spar design

Figure 6.16: Two additional spar designs that utilized a one vertical web 


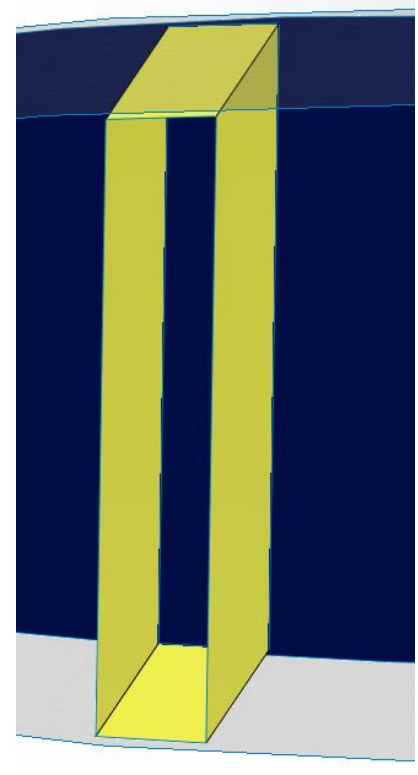

(a) Box spar design

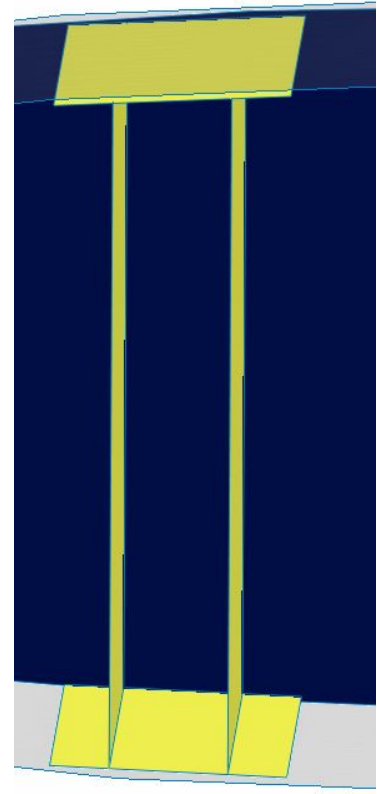

(b) Box with flanges spar design

Figure 6.17: Two additional spar designs that utilized a two vertical webs

Each spar design was imported into the same simulation model that was used for the validation of the $\mathrm{C}$ - channel design that was tested. In order to compare the designs, the strains on top of the leading edge were examined. All wing models showed a steady linear decrease of strain along the leading edge. The strain distribution for each spar design can be seen in Fig.( 6.18). Due to this trend it was decided that it would be reliable to compare each design based on the strain gage location that was closest to the fixed end. This location was 2.5 inches away from the root edge of the wing and on top of the leading edge spar. The force that was simulated on each of the designs was also held constant, and matched the experimental testing. Note that for some of the designs there was local compression at the end of the wing causing negative strains. 


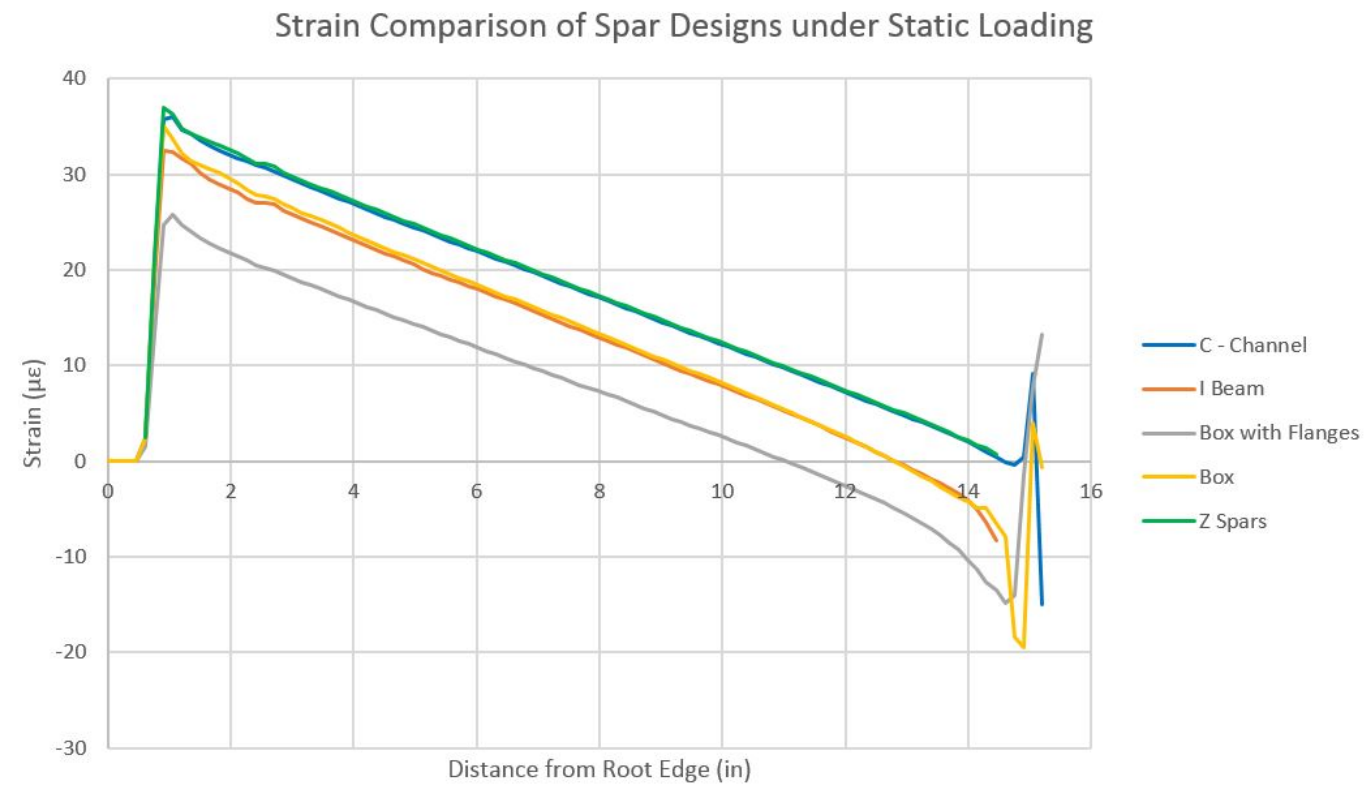

Figure 6.18: Strain distribution along the leading edge for each spar design simulated

It can be seen from the curves that the C- channel and the Z - spars had similar strain values. This could be because both of these feature similar designs except for one difference. The $\mathrm{Z}$ - spars have one flange on either side of the web, while the other has the flanges on the same side. The difference in strain between both designs was small, showing that the design difference did not have much of an effect on it stiffness. These two designs were the least stiff, since they had the highest strains compared to the others.

Two other designs that also had very similar strain curves were the I beam and the box designs. These two designs were similar in the sense that the total thicknesses of the webs were same. The difference is that the box beam used two webs that were half the thickness of the I beams single web. This shows that the box beam had no design advantage over the I beam in a bending situation. A possible advantage of the box beam could be in torsional resistance, since it has two webs spaced out increasing its width. I beam spars were manufactured as prototypes, and it was noticed that they were susceptible torsional forces. These 
two designs did show a reduction of strain of about $4 \mu \epsilon$ compared to the $\mathrm{C}$ channel and Z - spar designs.

The design that resisted the bending force the best was the box with flanges, which had flanges that extended past its webs. This design featured two webs like the box, although they were placed so that 0.125 inches of flange would extended past each side. This design showed a considerable strain reduction of $10.8 \mu \epsilon$, compared to the first two designs covered. The results from each of the designs can be seen in Table (6.2), in order of weakest to stiffest. A possible reason for this design performing better than the box, which was similar, could be due to bringing the webs closer together. This allowed it to use the advantages of the I beam and the box beam. It retained the advantages of having flanges, while also utilizing the two vertical web design. Having the two webs closer together could have increased its torsional stiffness even more, although this topic was not covered in this study.

Table 6.2: Summary of strain results from the 5 different spar designs simulated with the FEA model

\begin{tabular}{|c|c|}
\hline & Strain $(\mu \epsilon)$ \\
\hline Z - Spars & 31.1 \\
\hline C - Channel & 30.8 \\
\hline Box & 27.7 \\
\hline I Beam & 27.0 \\
\hline Box with Flanges & 20.3 \\
\hline
\end{tabular}

From this study it can be concluded that the best design for a static bending force would be a box beam with flanges. This design showed strain reductions of $35 \%$ compared to the $\mathrm{C}$ - channel design, that was made as a physical model for experimental testing. 


\subsection{Fatigue Simulation Results}

In order to model the fatigue life of the wing models, a stress life curve of the material was needed to show how the material behaves at varying stress levels. Due to large number of samples needed to experimentally test for the stress life curve of each material, a different method was investigated. An S-N curve for a carbon composite in terms of the percent of ultimate strength vs cycles to failure was applied to both of the materials. This was possible since the failure strength of the materials used in the project were tested for. The model used can be seen in Fig.( 6.19) below, along with fatigue behavior of other materials.

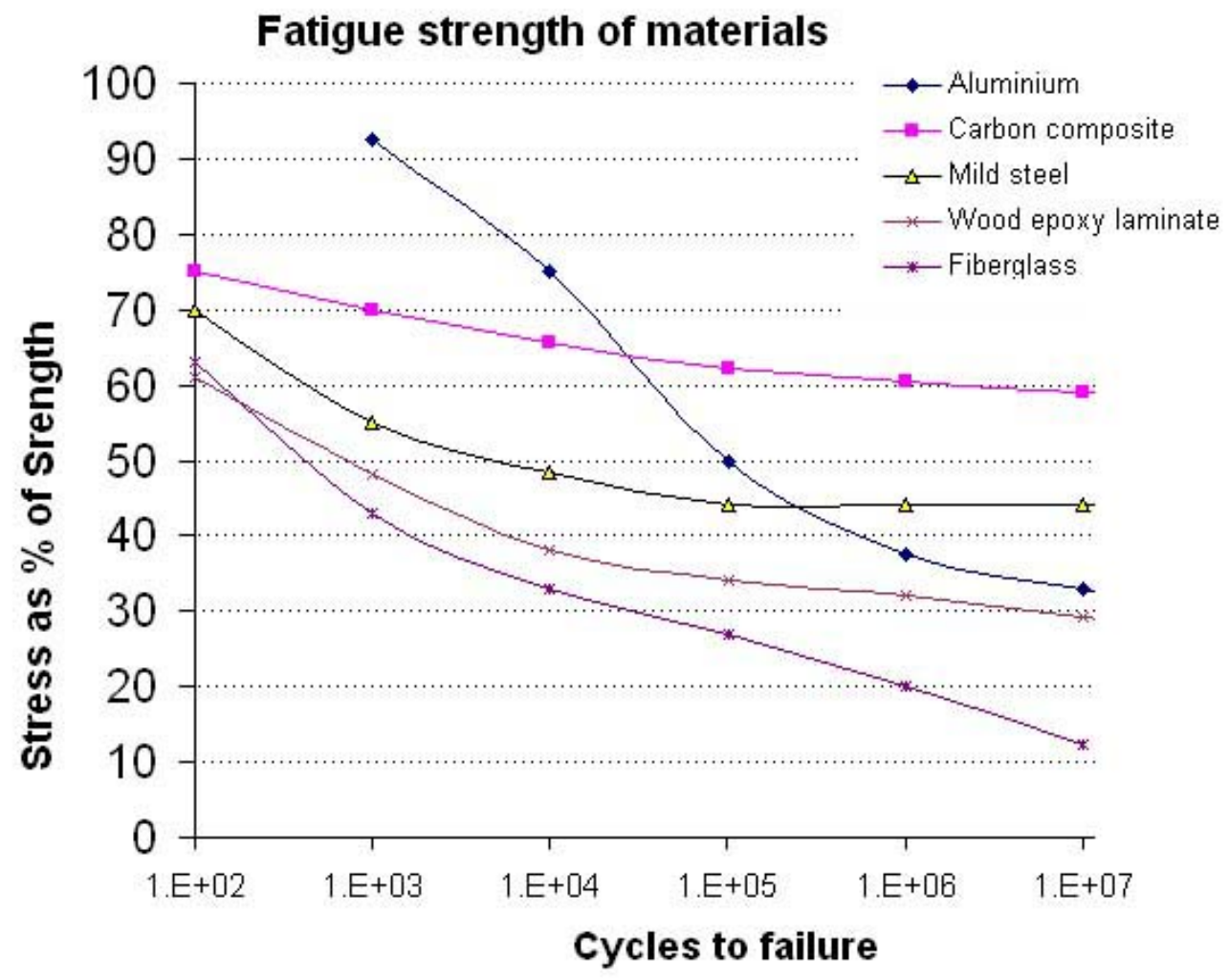

Figure 6.19: Stress life curve model applied to both carbon fiber materials used in this paper [38]

Using the fatigue tool in ANSYS, a cyclic displacement was put on the wing models in the same manner as the experimental testing. Everything else in the 
FEA model was held constant including the mesh, boundary conditions and material properties. In order to build the S-N curve for each wing, different displacement amplitudes were simulated on the wing models and plotted vs the number of cycles till failure. These steps were repeated for the each of the spar designs, so that it could be determined which would be the most fatigue resistant.

For all of the designs except for the $\mathrm{Z}$ - spars it was found that initial failure would occur at the tip of the wing. The joint where the spar and tip rib met was an area where the stress was higher than in other areas. This could be due to the sharp edges that are present in this area. It could also be attributed to the small thickness of the spars that was connected to the tip rib. An example of this stress concentration can be seen in Fig.( 6.20), where the location is shown by the red shading on the model.

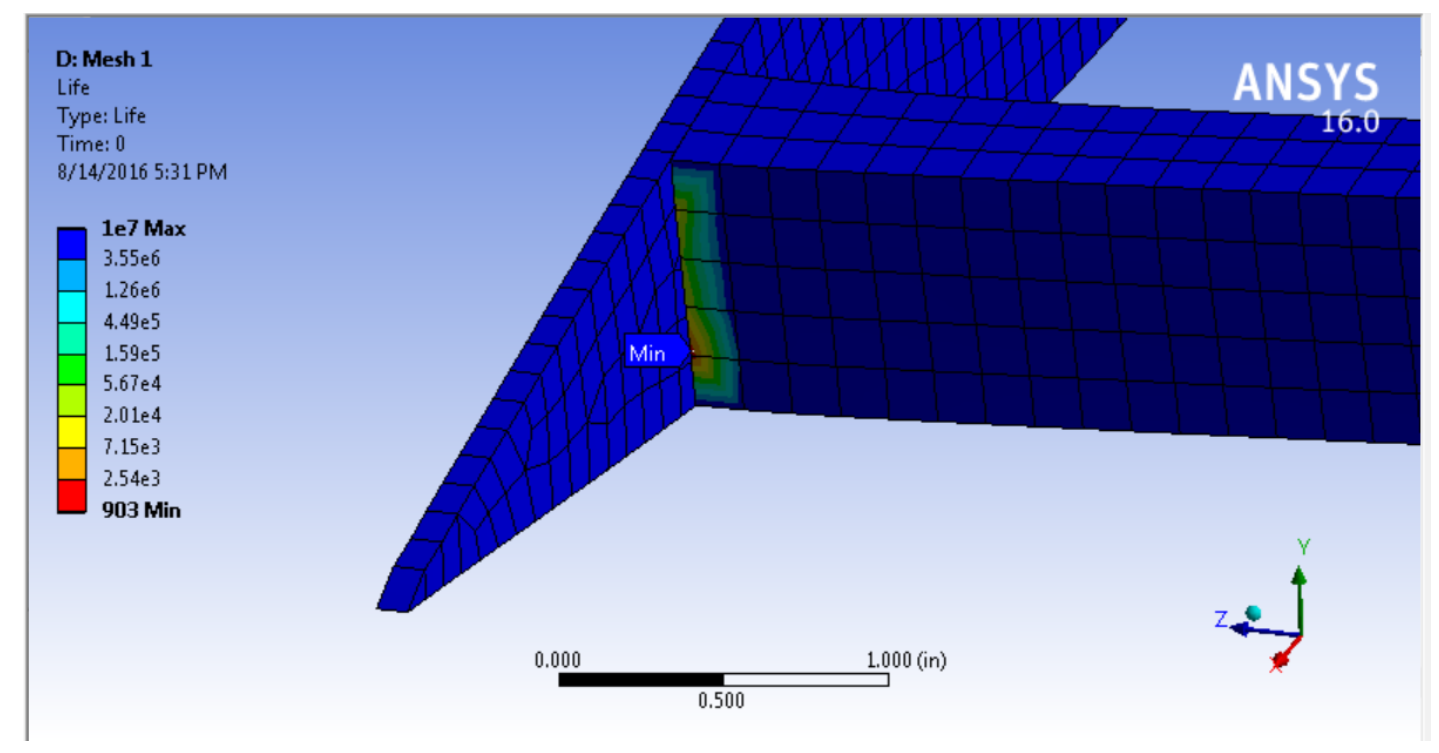

Figure 6.20: Location where most of the spar designs showed initial failure

The Z - spar design was unique in that the highest stress was at the leading edge spar, at the wing tip. This could have been caused by having both of the bottom flanges facing outward and the top inward. When the wing was displaced upward, it could have caused this location to buckle inward because of its instability. The 
$\mathrm{C}$ - channel design did not experience this problem since both of its spar flanges were facing inward making it stable for bending loads. The location where the $\mathrm{Z}$ - spar design had initial failure, can be seen in Fig.( 6.21).

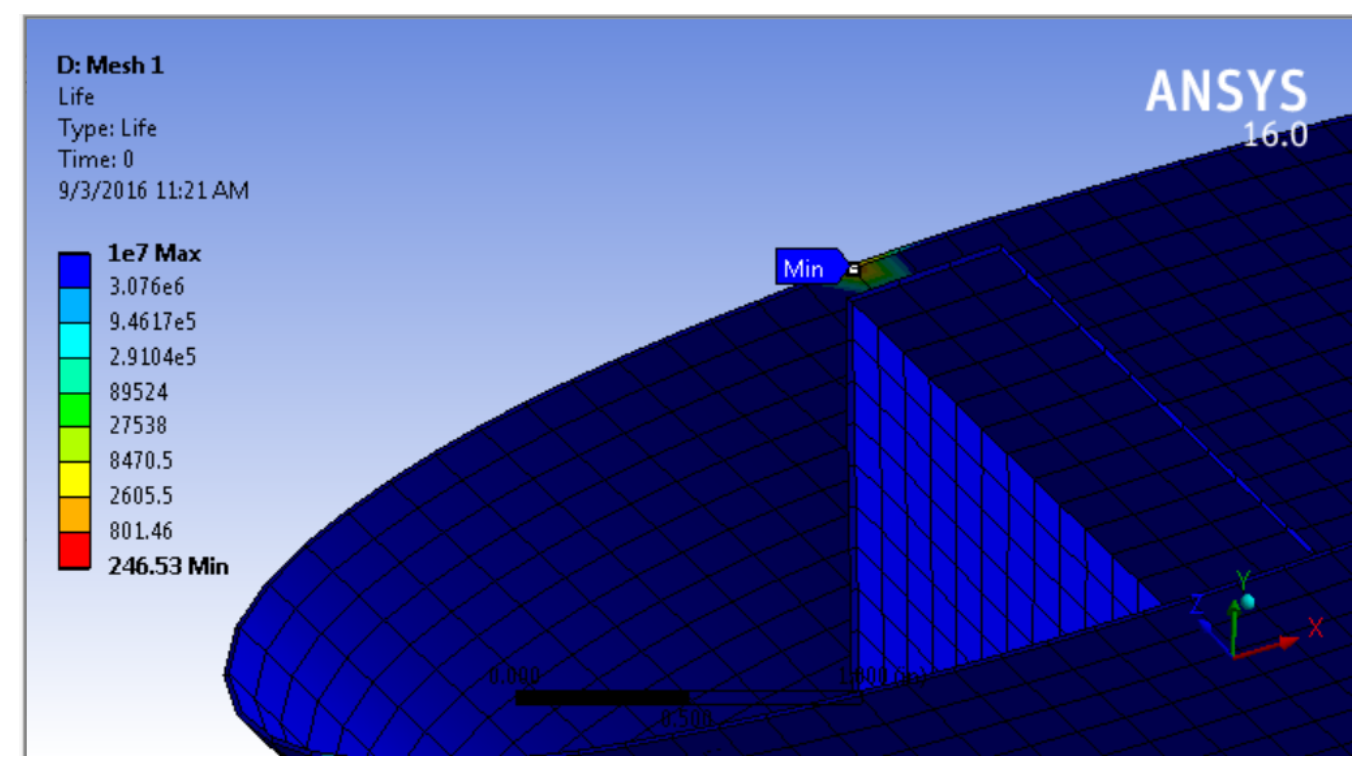

Figure 6.21: Location where the $\mathrm{Z}$ - spar design showed initial failure

After all the results for each of the designs were recorded, stress life curves for each were plotted together. This made it easy to distinguish which were the most fatigue resistant. From the plot it can be seen that the curves that were higher, were more resistant since they could withstand a higher displacement amplitude, and survive the same amount of cycles. The plot showing all of the designs stress life curves can be seen in Fig.( 6.22) 


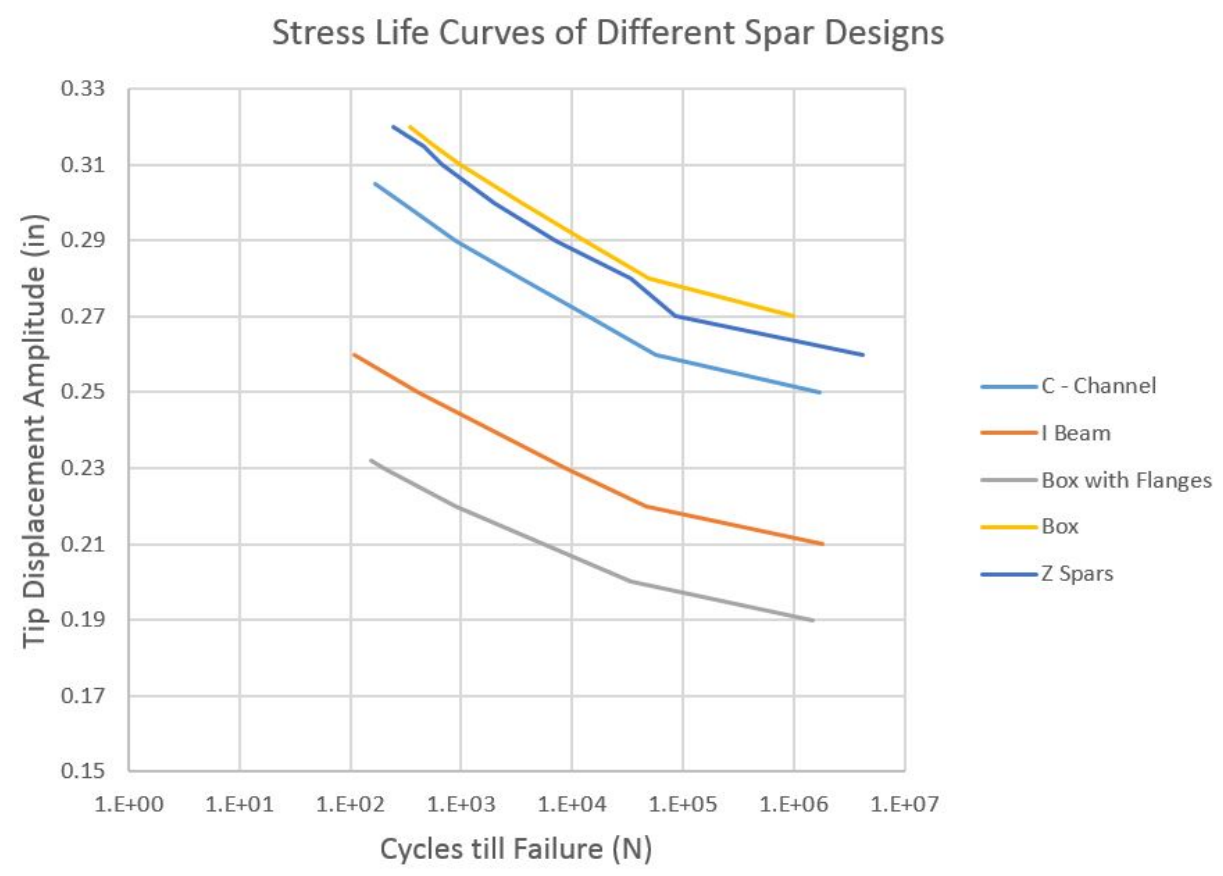

Figure 6.22: Stress life curves of each spar design

It can be seen that the two highest curves, meaning that they were most fatigue resistant are the box design and the $\mathrm{Z}$ - spars. What was interesting is that these designs were the least stiff in the static force study. This could attributed to the design's flexible nature. The flexibility in the wing would have allowed for it to take a far greater amount of cycles. In this case when designing a structure that would need fatigue resistance, it is important to find a balance between strength and flexibility. A rigid structure might be better suited for a high load but not for repeated small loads or displacements.

The curves of the I beam and box with flanges, show this trend since they were the stiffest designs from the static study, but did not fare well in the fatigue simulations. The box with flanges design could only withstand two thirds of the displacement amplitude as the box design, for the same amount of cycles till failure. An optimal design would need to be chosen based on the application of structure and its projected loadings. 
Chapter 7

RESULTS COMPARISON AND CONCLUSION

In this chapter the model validation results from the experimental testing, FEA model and theoretical calculation are compared. The next section compared the static test results for the wing model. Then the main results from this paper were outlined in the conclusion section. Improvements to this thesis are presented in a future work section.

\subsection{Model Validation Results Comparison}

From the FEA results for the aluminum beam it was found that the strains at 4" and 8" were $-164.4 \mu \epsilon$ and $-133.1 \mu \epsilon$ respectively. The difference between these results and the experimental test were $4.4 \%$ and $4.2 \%$ respectively. From the plot in Fig.( 7.1) you can see that the data points from the other two methods match well with the results from the FEA simulation. The FEA data lies approximately between the data from the two other methods. The theoretical calculation results shown by the purple dots, had respective differences of $2.8 \%$ and $2.9 \%$ to the FEA results. The results for the strain at the two locations using the three methods are shown in Table ( 7.1). 


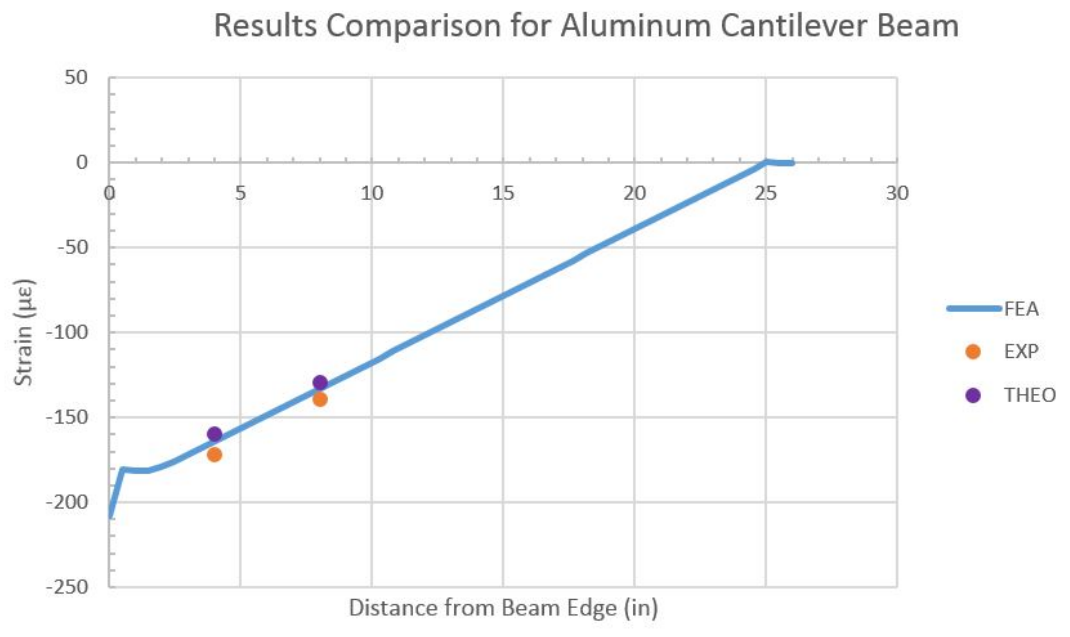

Figure 7.1: Strain distribution comparison along the aluminum cantilever beam

Table 7.1: Strain at strain gage location comparison for all three methods

\begin{tabular}{|l|c|c|c|l|l|l|}
\hline \multirow{2}{*}{ Gage $\#$} & EXP & FEA & THEO & \multicolumn{3}{|c|}{ Percent Error (\%) } \\
\cline { 5 - 7 } & $(\mu \epsilon)$ & $(\mu \epsilon)$ & $(\mu \epsilon)$ & Exp \& Theo & FEA \& Exp & FEA \& Theo \\
\hline 1 & -172 & -164.4 & -159.7 & 7.7 & 4.4 & 2.8 \\
\hline 2 & -139 & -133.1 & -129.3 & 7.5 & 4.2 & 2.9 \\
\hline
\end{tabular}

After calculating the maximum displacement using the theoretical equation, it was found that it would be 0.329 inches due to pure bending. This value was then compared to the FEA model and its result of 0.337 inches. These two values differed by $2.3 \%$, showing that they are in good agreement. The difference in these can be attributed to the FEA model being an approximate solution of the analytical calculation. A summary of the maximum displacement results for the two methods is in Table ( 7.2).

Table 7.2: Maximum displacement of aluminum beam comparison of FEA and analytical results

\begin{tabular}{|l|c|l|c|}
\hline & Theoretical & FEA & Percent Error (\%) \\
\hline Displacement (in) & 0.329 & 0.337 & 2.30 \\
\hline
\end{tabular}

This study shows that the simulation model made is a good predictor for not only the experimental test, but also for an analytical calculation. With these 
results we gain greater confidence in the boundary conditions and loadings in the FEA software.

After running the simulation for both beams made of the two different composite materials, the strains were compared to the experimental results. The strain data along the path was exported into an excel spread sheet, so that the numerical and experimental curves could be compared. In Table ( 7.3) and Table ( 7.4), the results at the strain gage locations for both methods for each material can be seen. Both simulations yielded close results to the experimental data. The average percent error of all the strain gages for the Cycom and Hexcel material were 4.6 $\%$ and $8.6 \%$ respectively. This showed that the FEA software correctly modeled the experimental test, and that the strain gages are working as intended. The Hexcel beam did show a slightly greater difference, which could have been due to not using the correct strain gage adhesive. Since this beam was tested before the recommended materials were ordered, these strain gages were bonded using super glue and 3M scotch tape. Using the correct adhesive allows for a quick and consistent cure, as well as a thin bond line. This slight difference in the curves between the experimental and FEA results, could have been due to not using the recommended practices by Micro Measurements. The strains along the beams made of both composite materials can be seen in the tables below.

Table 7.3: Summary of strains along the Cycom Cantilever Beam

\begin{tabular}{|c|c|c|c|}
\hline Distance (in) & EXP $(\mu \epsilon)$ & FEA $(\mu \epsilon)$ & Percent difference $(\%)$ \\
\hline 1.5 & 828.3 & 762.1 & 8.0 \\
\hline 2.5 & 766.5 & 684.5 & 10.7 \\
\hline 4 & 601.0 & 564.0 & 6.2 \\
\hline 5.5 & 440.5 & 443.1 & 0.6 \\
\hline 7 & 311.6 & 322.3 & 3.4 \\
\hline 8.5 & 205.3 & 201.4 & 1.9 \\
\hline 10 & 82.0 & 81.0 & 1.3 \\
\hline & & Average & 4.6 \\
\hline
\end{tabular}


Table 7.4: Summary of strains along the Hexcel Cantilever Beam

\begin{tabular}{|c|c|c|c|}
\hline Distance (in) & EXP $(\mu \epsilon)$ & FEA $(\mu \epsilon)$ & Percent difference $(\%)$ \\
\hline 1.5 & 359 & 331.8 & 7.6 \\
\hline 2.5 & 310 & 297.2 & 4.1 \\
\hline 4 & 270 & 244.8 & 9.3 \\
\hline 5.5 & 211 & 192.3 & 8.8 \\
\hline 7 & 154 & 139.9 & 9.2 \\
\hline 8.5 & 90 & 87.4 & 2.9 \\
\hline 10 & 43 & 35.2 & 18.2 \\
\hline & & Average & 8.6 \\
\hline
\end{tabular}

When the theoretical results were compared to the FEA results, it was found that the Cycom material matched well but the Hexcel did not. This comparison can be seen in Fig.( 7.2), where the theoretical calculations are shown as a purple circle. The average percent difference between experimental and numerical for the Cycom and Hexcel materials were $4.6 \%$ and $41 \%$ respectively. The reason that the theoretical calculation did not match for the Hexcel is because the FEA simulation used the measured modulus from the material testing. Although, since the FEA model arrived at similar results as the experiment it can be concluded that it is validated. The theoretical calculation used the modulus from the data sheet which differed from the cured specimens.

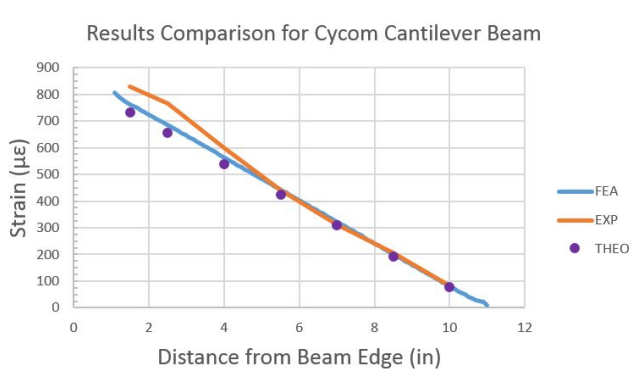

(a) FEA and experimental strain curves for the Cycom material

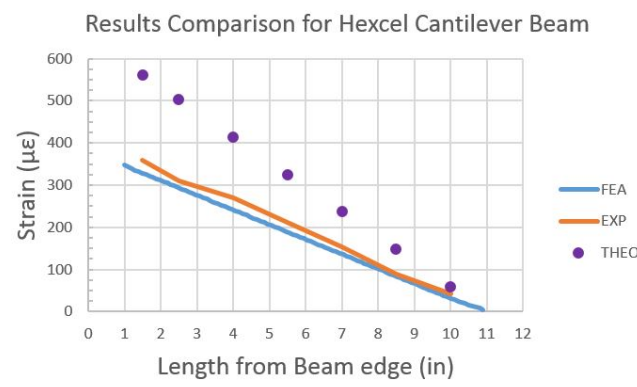

(b) FEA and experimental strain curves for the Hexcel material

Figure 7.2: Strains along the cantilever beams for both materials used in wing model

Since the tested modulus of the Cycom material was close to the data sheet 
both of the methods yielded similar results. This also shows the simplification of the othrotropic equation to an isotropic equation is correct, since it matches the FEA software. If the FEA model for the Hexcel would have used the modulus from the data sheet, it would have had very close results to the theoretical.

The minimal differences for the Cycom material can be attributed to an unstable load during the measurement, load placement, or material properties. Although, the final percent differences were small enough, that the model can be used for simulation. With this confidence in the testing and FEA modeling methods, the actual wing model would then be analyzed in the ANSYS software.

\subsection{Static Simulation Results Comparison}

Then the static test results of the wing for the experimental method and the FEA simulation were compared for the static loading. The purpose of this study was to investigate whether the FEA model would be a good representation of the testing. If the results match, it would allow more spar designs to be simulated in the FEA model, without making a physical model. The benefits of not having to make a physical model, are cost as well as manufacturing and assembly time savings.

When comparing strain values from the two methods, it was found that for the leading edge group the results were similar. It can be seen in Fig. ( 7.3) that the curves for each wing, and the FEA followed the same trend and are close in magnitude. Since the percent differences varied strain gage to strain gage the average was taken for each wing. The average percent difference for each wing was $17.5 \%, 8.7 \%$, and $7 \%$ which is lower than the rest of the groups. Since strain gages were not placed along the whole length of the wing, the experimental curves only had data from 2.5 inches until 10.5 inches. On the other hand there are strain results from the FEA model along the whole length of the wing. Between the wings 
it was observed that two and three had close results to each other, as well as the FEA results. Wing one measured slightly lower strains along its leading edge spar. These variations were small but could possibly be attributed to the assembly of the wings. The carbon fiber materials used could have also cured differently for various parts. Overall due to the small percent differences, the FEA model would be able to simulate the experimental data for this group.

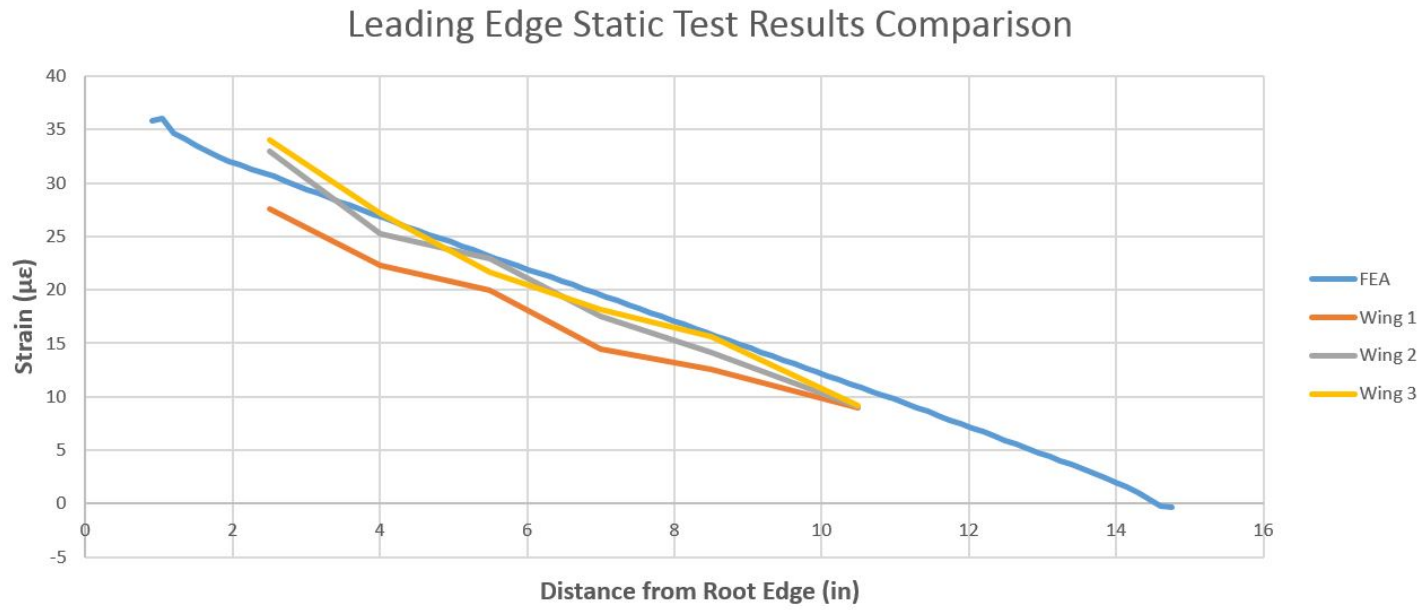

Figure 7.3: Strain distribution comparison along the leading edge spar

The next group of interest was the trailing edge spar, which had its strain gages at the same locations span wise than the leading edge group. For this area the strain results between the two methods differed by a greater amount than the previous. The percent differences for each wing to the FEA model were $44.9 \%$, $24.3 \%$, and $54.5 \%$ respectively. The differences in strain values can easily be seen in the plot in Fig.( 7.4). 


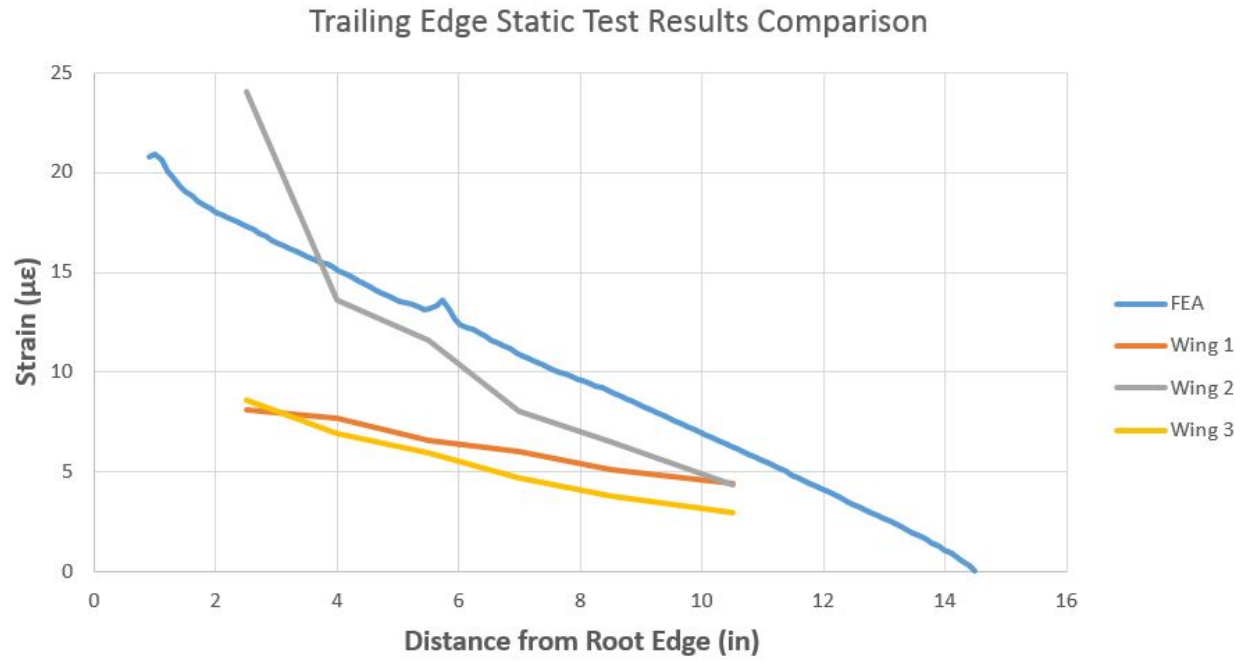

Figure 7.4: Strain distribution along the trailing edge spar for FEA and experimental data

Wings one and three showed similar results, although they were far lower than the FEA model strains. A possible explanation for the greater strain could be that excessive adhesive was used in these areas compared to wing two. Wing two showed strain values that were closer in magnitude to the FEA result, although it is still not in an ideal range to have great confidence in the simulation. Another reason for the low strains from the experimental test could be that the boundary condition issue affected this area more than the leading edge. The bump in the wing skin could have caused enough discontinuity at the boundary condition in this area to change the load path of the wing.

If the wing was not fully constrained in this area the wing would displace without resistance at the constraint. Due to movement the stress, and in turn the strains, would be lower than they would be expected to be.

Another issue is that the FEA model treats all contact areas as perfect bonds. Although in reality these locations are bonded joints that are not perfectly fixed. In order to model the adhesive it would have required extensive testing of the adhesive used. Then the properties would have to be implemented into the simulation 
model.

Due to the difference in the results there is not much confidence in the results from this group. The FEA simulation showed good results for the leading edge but when comparing the trailing edge strains issues arose.

The last group that was investigated in this study, was the chord-wise location. This was the group that had the least agreement between the experimental and FEA results. Some of the strain gages displayed similar results between the methods, but most differed by a substantial amount. A small deviation in strain gage angle could have caused the strain value to vary greatly, compared to the spar strain gages which were lined up. The strain gages might also have not been lined up at the same distance away from the fixed end.

The FEA model had higher strains between the spars than what was actually measured. This would lead me to believe that in the simulation the skin is carrying a significant portion of the load. Where in the physical model perhaps the load flexes in this area relieving the strain. Another explanation could have been that the wing was not fully constrained. If the constraint between the two spars on the physical model is not adequate, the structure would be allowed to displace in this location. Therefore it would register a low strain measurement since it is not resisting a force.

As explained the FEA model treats every bond as fixed locations which provides heavier loaded paths throughout. For this reason the chord and trailing edge locations did not have as much strain as the model. The bonds could have allowed for some displacement reliving the stress in these areas.

The average percent differences for each tested wing to the FEA results were all greater than 35\%, therefore results from the simulation for this area are not trusted. Any further studies conducted with this simulation model would focus on the leading edge spars. A comparison of the three wings along with the FEA 
results can be seen in Fig.( 7.5) below.

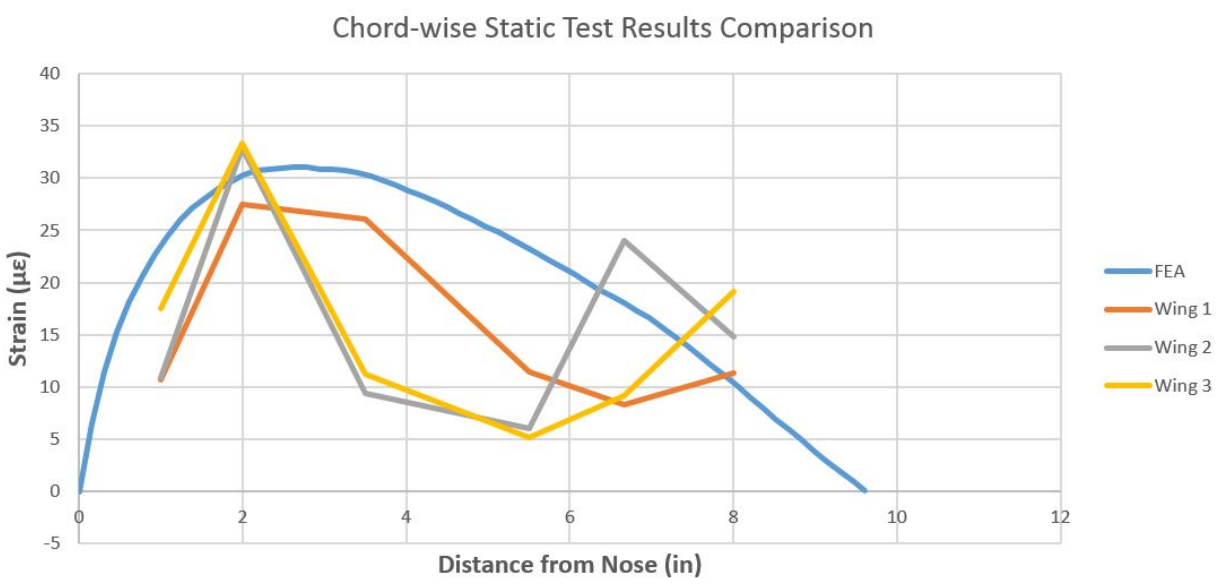

Figure 7.5: Strain distribution in the chord-wise direction for FEA and experimental data

It was found through fatigue simulations that the displacement amplitude used in the experimental testing, was low compared to a magnitude that would cause damage. The amplitude used in the testing was 0.03 in and all three wings ended up not experiencing any damage. This displacement was found to impart an estimate of about $5 \mathrm{lbf}$, which was believed to be a considerable amount. There was also a risk of prematurely failing the wing by increasing the displacement.

When the FEA simulation for the C- channel design was run for this displacement, it was found that the wing would survive more than a million cycles. Therefore the simulation showed the same result for the fatigue testing, since the wing model survived 100,000 cycles. When the displacement amplitude was increased to 0.26 inches the cycles to failure was decreased to 57 thousand cycles. If the testing was done at this magnitude then the failure could have been seen, since the cycle limit was up to 100 thousand cycles. When the simulation was done for a displacement of 0.305 inches then the structure would only take 170 cycles till failure. When a displacement of 0.28 inches which was approximately between the latter two was used, it would last 3500 cycles. It can be seen that the number of 
cycles decreased substantially due to this increase in amplitude.

It was difficult to predict what amount of displacement to be used in the testing since only a few models were made. Fatigue behavior is non linear and a small increase in amplitude led to far lower cycles that it could withstand. In order to better investigate the fatigue failure through experimental testing, numerous samples would need to be made. Since the wing model took a considerable time to make, it was not possible to make many specimens.

\subsection{Conclusion}

In this study, a composite wing model was made in order to investigate static and fatigue bending resistance. A finite element model was implemented to simulate other spar designs after validation.

First task was the static testing. Experimental test results for carbon fiber cantilever beams were similar to theoretical and FEA calculations. Strain measurements along the leading edge of the wing matched with the FEA simulation by $9.8 \%$. Agreement between the experimental and FEA results was dependent on the experimental fixture. Strain gages on the wing helped to study the deformation of the wing. The box with flange spar design was the stiffest from all the designs simulated with the FEA model.

Second task was the fatigue testing. The test frequency had a minimal effect on the strain results. Since it was minimal, it was advantageous to increase the frequency to reduce the test duration. All wing models survived a $0.03 "$ displacement amplitude for 100,000 cycles without damage. From the fatigue simulation model, it was determined that the box spar design was the most resistant to bending fatigue. The fatigue test from the FEA model were largely influenced by wing stiffness. 


\subsection{Future Work}

Future work should utilize a jig, in order to insure strain gages are orientated as accurate as possible. Using consistent bond lines between parts could also decrease some of the variability between different wing models. It would also be beneficial to test the wing models at a higher displacement amplitude, so that the life of the structure could be compared to the simulation. It is recommended that one of the additional wing designs be made, so that it could be determined if the FEA model can be used for predictions. 


\section{BIBLIOGRAPHY}

[1] History of Composites. about money. 2016. http://composite.about.com/od/ aboutcompositesplastics/a/HistoryofComposites.htm.

[2] V. Ryan. Composite Materials - Concrete. Technology Student. 2010. http:// www.technologystudent.com/joints/concret1.html.

[3] carbonfiberglass. 2010. http://images.carbonfiberglass.com/image/data/wpc /2010/11/hand-lay-up-300x259.jpg.

[4] Advanced Composite Materials. faa. http://www.faa.gov/regulations ${ }_{p}$ olicies/

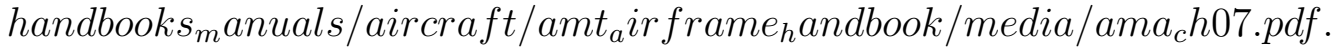

[5] Falzon Brian. The Airbus A380 wing cracks: an engineer's perspective. The Conversation. 2012. http://theconversation.com/the-airbus-a380-wing-cracksan-engineers-perspective- 5318 .

[6] John Tomblin. Waruna Seneviratne. Determining the Fatigue Life of Composite Aircraft Structures Using Life and Load-Enhancement Factors. Air Traffic Organization. 2011.

[7] Ali Movaghghar. Gennady Ivanovich Lvov. Theoretical and Experimental Study of Fatigue Strength of Plain Woven Glass/Epoxy Composite. Kharkov State Polytechnic University, Faculty of Mechanical Engineering, Ukraine. 2012.

[8] Nyman Tonny. Fatigue and Residual Strength of Composite Aircraft Structures. Department of Aeronautics Kungliga Tekniska Högskolan (Royal Institute of Technology). 1999.

[9] Kopeliovich Dmitri. Fatigue. SubsTech. 2003. http://www.substech.com/doku wiki/doku.php?id=fatigue. 
[10] Bak Michael. Engineering Advantage - Mean Stress Corrections in Fatigue. CAE Associates. 2016. https://caeai.com/blog/mean-stress-corrections-fatigue.

[11] Warp and woof. Wikipedia. 2016. https://en.wikipedia.org/wiki/Warp ${ }_{a} n d_{w}$ oof.

[12] Aminmi Ahmad. Static and Fatigue Failure Response of Woven Carbon Fiber Specimens with Double-Edge Notches. California Polytechnic State University, Aerospace Engineering, San Luis Obispo. 2010.

[13] STOL CH 701 Construction. Zenith Aircraft Company. 2014. http://www.zenit hair.com/stolch701/7-construc.html.

[14] Rapid Prototyping. Stratasys. 2016. http://www.stratasys.com/solutions/rapidprototyping.

[15] Chemlease 41-90 EZ Semi-Permanent Release Agent. ChemTrend. December 2004.

[16] deLuna Richard. Effect of Low Velocity Impact on the Vibrational Behavior of a Composite Wing. California Polytechnic State University, Aerospace Engineering, San Luis Obispo. 2016.

[17] System 8000 StrainSmart Data Acquisition System. Micro Measurements. 2015.

[18] markopolo. Tripp Lite Inverter/Charger - How to make a remote switch. Class B Forums. 2006. http://www.classbforum.com/forums/f8/tripp-liteinverter-charger-how-to-make-a-remote-switch-18.html.

[19] The Three-Wire Quarter-Bridge Circuit. Micro Measurements. 2015.

[20] Model 8000-8-SM Instruction Manual. Micro Measurements. 2014.

[21] Shunt Calibration of Strain Gage Instrumentation. Micro Measurements. 2013. 
[22] D3039: Standard Test Method for Tensile Properties of Polymer Matrix Composite Materials. ASTM. 2008.

[23] Standard Test Method for Poisson's Ratio at Room Temperature. ASTM. 2010.

[24] Alexander Ron. Basics of Composite Construction. Alexander SportAir Workshops. 1999. http://exp-aircraft.com/library/alexande/composit.html.

[25] Cycom 7714A Epoxy Resin. Cytec Engineered Materials. 2012.

[26] HexPly 8552 Product Data. Hexcel Composites. 2013.

[27] LOCTITE EA 956 AERO Epoxy Paste Adhesive. Loctite. 2013.

[28] Standard Test Method for Ignition Loss of Cured Reinforced Resins1. ASTM. 2002.

[29] Fibre Content. Quinn. 2002.

[30] Al Makky Ahmed. Computational Fluid Dynamics is the Future. Alexander SportAir Workshops. 2013. http://cfd2012.com/matlab-beam-analysis.html.

[31] Kolkailah Faysal. Dr. Kolkailah's Notes Composites. California Polytechnic State University, Aerospace Engineering, San Luis Obispo. 2013.

[32] 8801 Series Fatigue Testing Systems. Instron. 2005.

[33] Aluminum 6061-T6; 6061-T651. ASM Aerospace Specification metals Inc. http://asm.matweb.com/search/SpecificMaterial.asp?bassnum=MA6061t6.

[34] Timins A. R. Test and space experience with protoflight spacecraft. NASA. 1995. https://ntrs.nasa.gov/search.jsp?R=19760032840.

[35] Omega. Practical Strain Gage Measurements. Omega. 1999. http://www.omeg

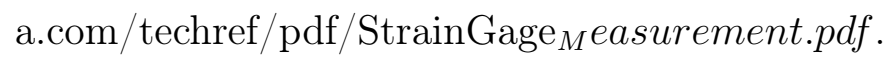


[36] FE-SAFE DURABILITY ANALYSIS SOFTWARE FOR FINITE ELEMENT MODELS. Simulia. 2016. http://www.3ds.com/products-services/simulia/pro ducts/fe-safe/.

[37] Using ANSYS ACP for Innovative Composite Part Analysis - CAE Associates - ANSYS e-Learning. CAE Associates. 2014. https://www.youtube.com / watch? $\mathrm{v}=\mathrm{udmLaBKx} 7 \mathrm{rA}$.

[38] MikeJohns. Material strength and fatigue. boatdesign. 2004. http://www.boat design.net/forums/boat-design/material-strength-fatigue-13174.html. 


\section{APPENDICES}

A. Model 8000 DAQ IP Address Setup

Setting up IP address so that the DAQ can communicate with Computer Make sure Strain Smart software is installed. It will be needed after these steps are finished

1. Without having DAQ connected or powered on click on control panel - Network and Sharing Center - Change adapter settings

2. Right click on any wireless connection and click on disable

3. Right click on Local Area Connection - Properties

4. Click on Internet Protocol Version 4 - Properties

5. Click on "Use the following IP address" bubble

6. Manually enter the IP address: 192.168.0.2 ** If the Subnet mask automatically filled its self in then this set is done correctly. If it didn't its because full administrative rights are not being allowed on the computer. $* * *$

7. Click OK on both windows to go back to where the Local Area Connection button is on the Network connections window

8. Connect the Ethernet cable from the computer to the DAQ *** Make sure to plug it in where it says Ethernet next to the power switch on the DAQ******* 
Make sure not to plug it into the "relay" port on the back ******

9. Then power on the DAQ with the switch and you should see it change from "Network cable unplugged" to something like "identifying"

10. If it connects then you are now ready to start the strain smart software to start a new project

B. Model 8000 DAQ Connections and Strain Smart Setup

First you will need to setup the IP address on the computer to be used by following the setup instructions preceding this one.

1. Connect the model 8000 power cable to the daq. The plug needs to be inserted and twisted till it locks in place.

2. Next connect an Ethernet cable from the computer for data collection to the model 8000 daq. Make sure that the port is labeled Ethernet and not relay as this two ports use the same type of cable. [20]

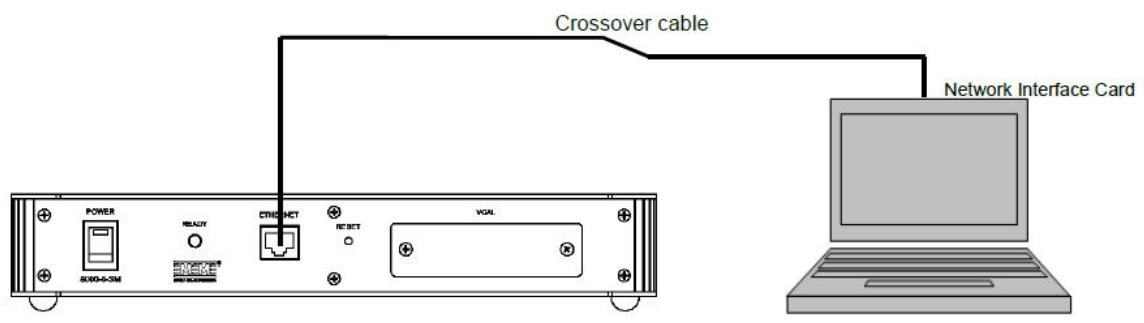

3. You can then begin to connect $1 \mathrm{ft}$ Ethernet cables to the back of the daq where the channels 1-8 are labeled. 
4. Next short Ethernet cables will be plugged into the RJ-45 circuit breaks out.

The breakouts make it easy to plug the leadwires from the strain gages to its screw type connections.

They are labeled from 1-8 for each individual wire in the cable.

This eliminates having to splice the ethernet cable.

The breakout labels also correspond to the diagrams below and in the manual for the system.

5. You can then plug in the wires from your strain gages to the breakouts according to the diagrams below.[20]

Note: If you are connecting a highlevel device such as the instron output signal then use the last connection shown.

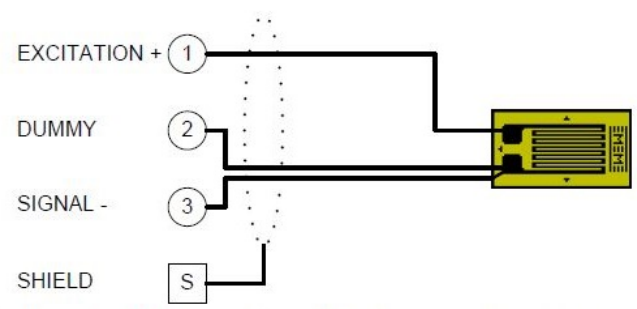

Quarter-Bridge /Three-Wire Connection (Internal Dummy)

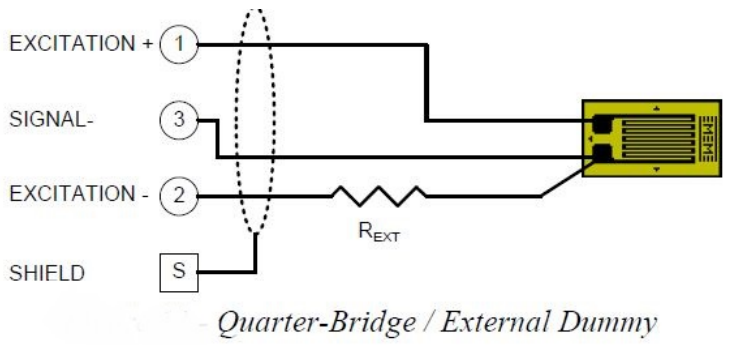




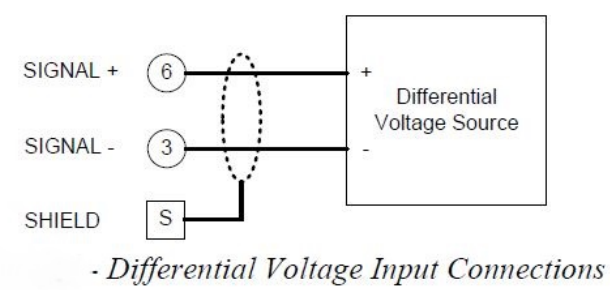

6. Next make sure your computers wifi is turned off.

7. Now open up the strain smart software and click on the new project button.

8. Name your project and the click on next

9. Next click on new sensor and from the top bar select the type of device that will be used for the channels.

These could be strain gages, thermocouples, and high level devices, etc

10. For a uniaxial strain gage type in the gage factor, and resistance in the window. 


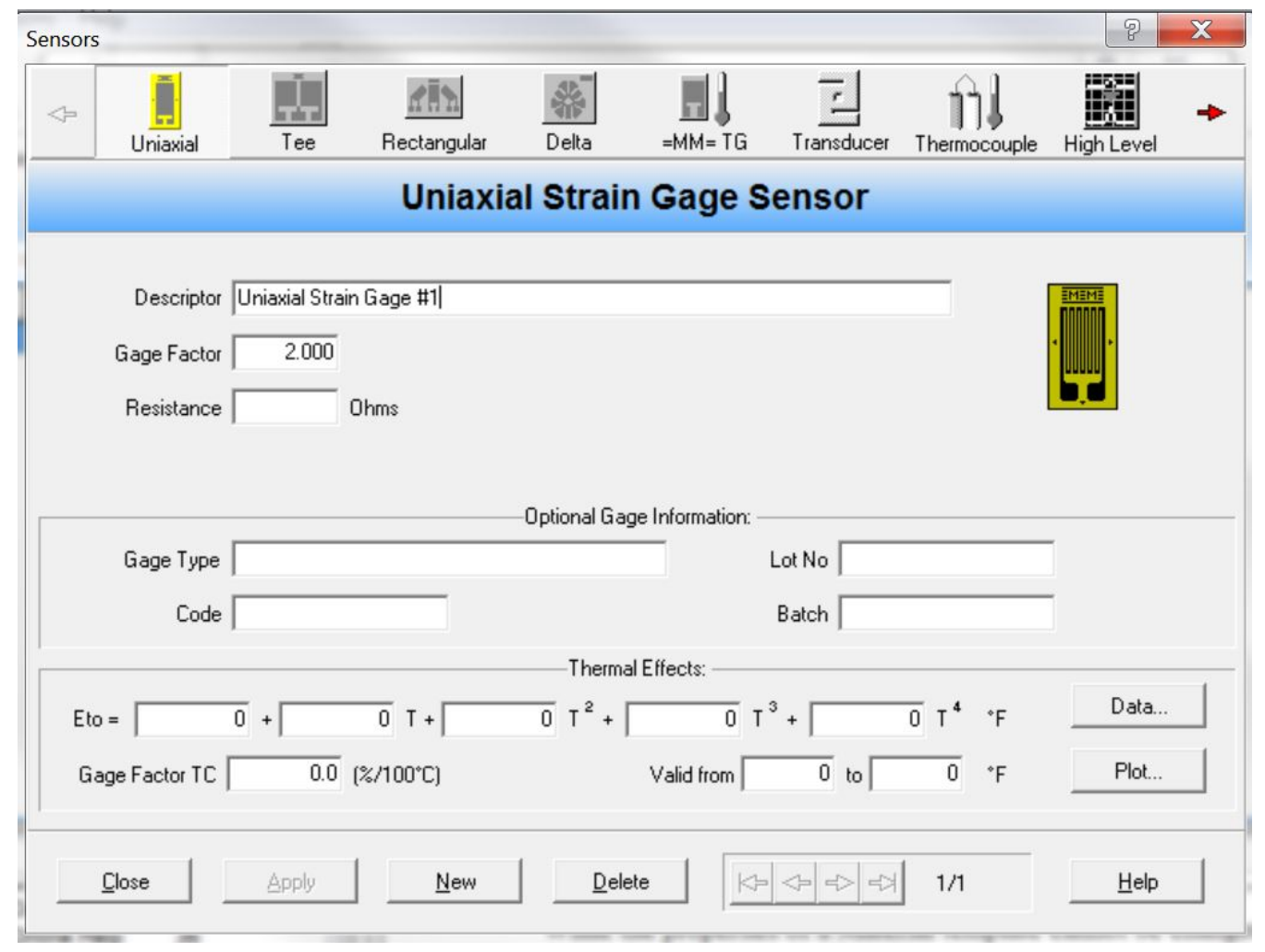

11. For a high level device select the output units and click on the calibration information button.

This will allow you to input the voltage that correspond to the physical unit that will be output form the instron controller through a BNC cable.

In the instron settings this calibration can be defined based on the limits of the sensor. 

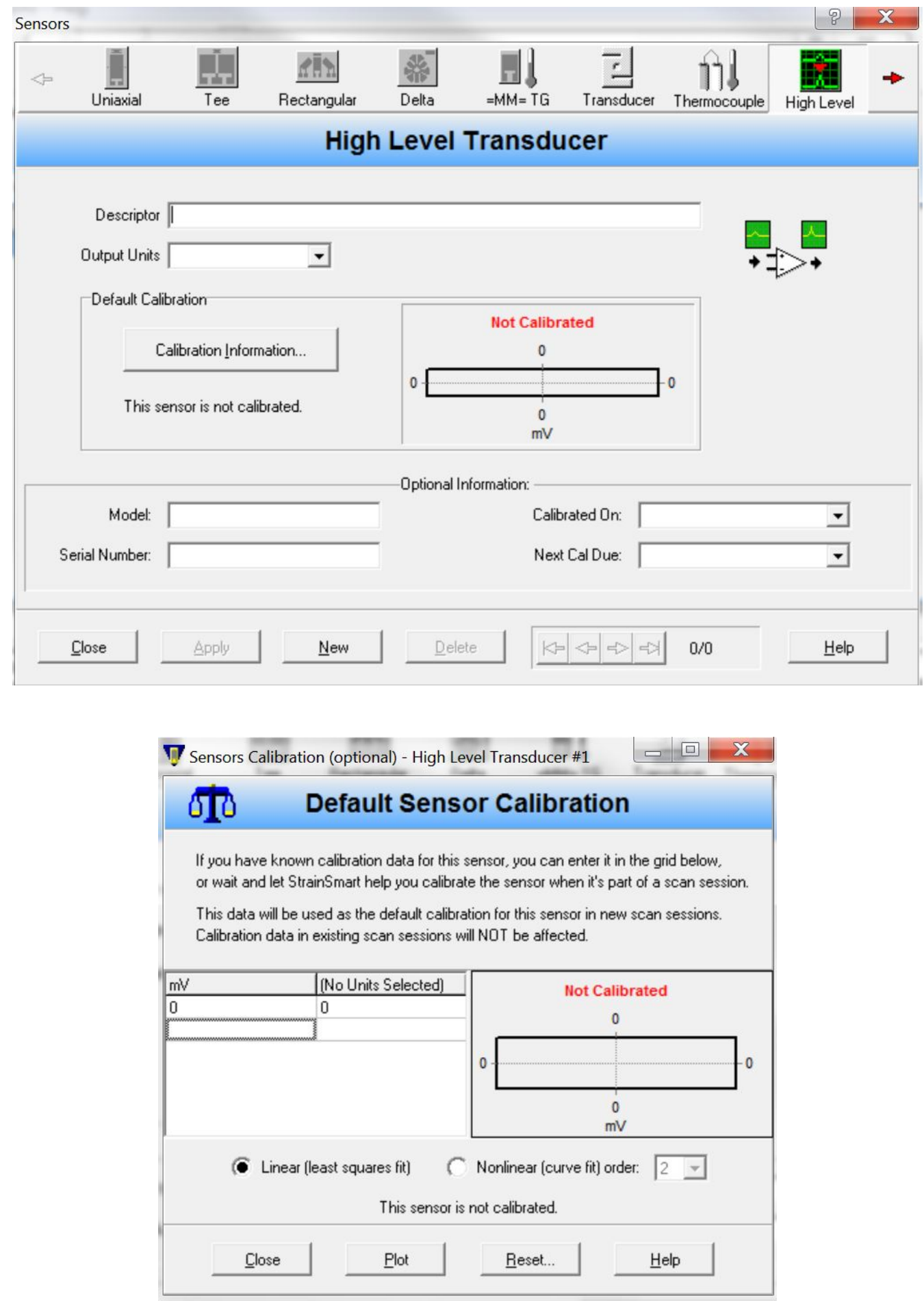

12. Next close the sensors window and click on next in the wizard window.

13. The next window in the wizard can be used to in put a material so that the software would calculate stress automatically. This works best for homogenous 
materials.

14. Click next to select what channels will be used for data collection.

Click on new channels and highlight the channels that have strain gages connected and press ok.

If a high level device is being used for the channel make sure to select the high level button before pressing ok.

Once the channels have been selected press next in the wizard.

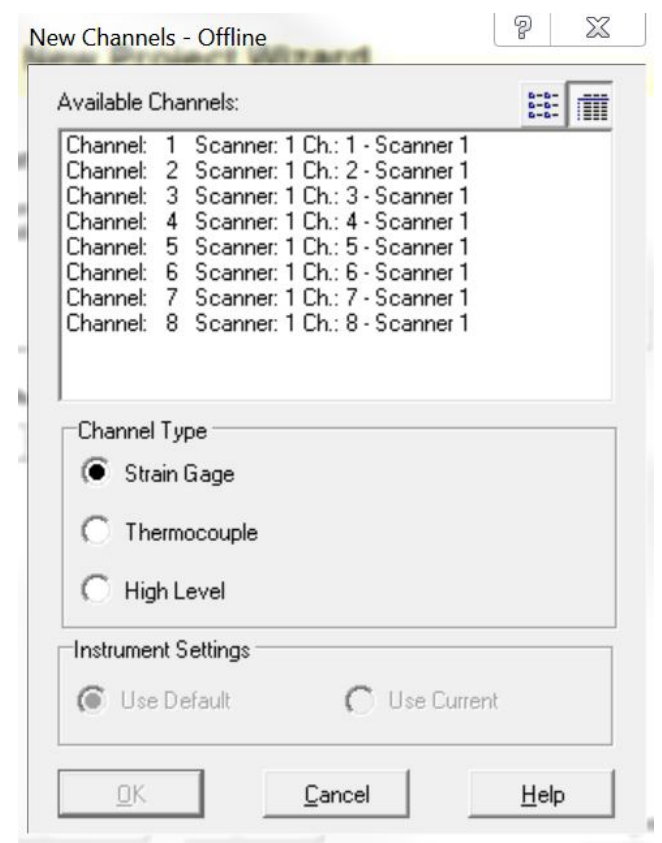

15. Next you will be assigning what type of sensor is being used on each channel. Click on new channel assignment to bring up the window.

In this window click on the sensor pull down tab and select the strain gage or high level device that was defined in earlier steps. 


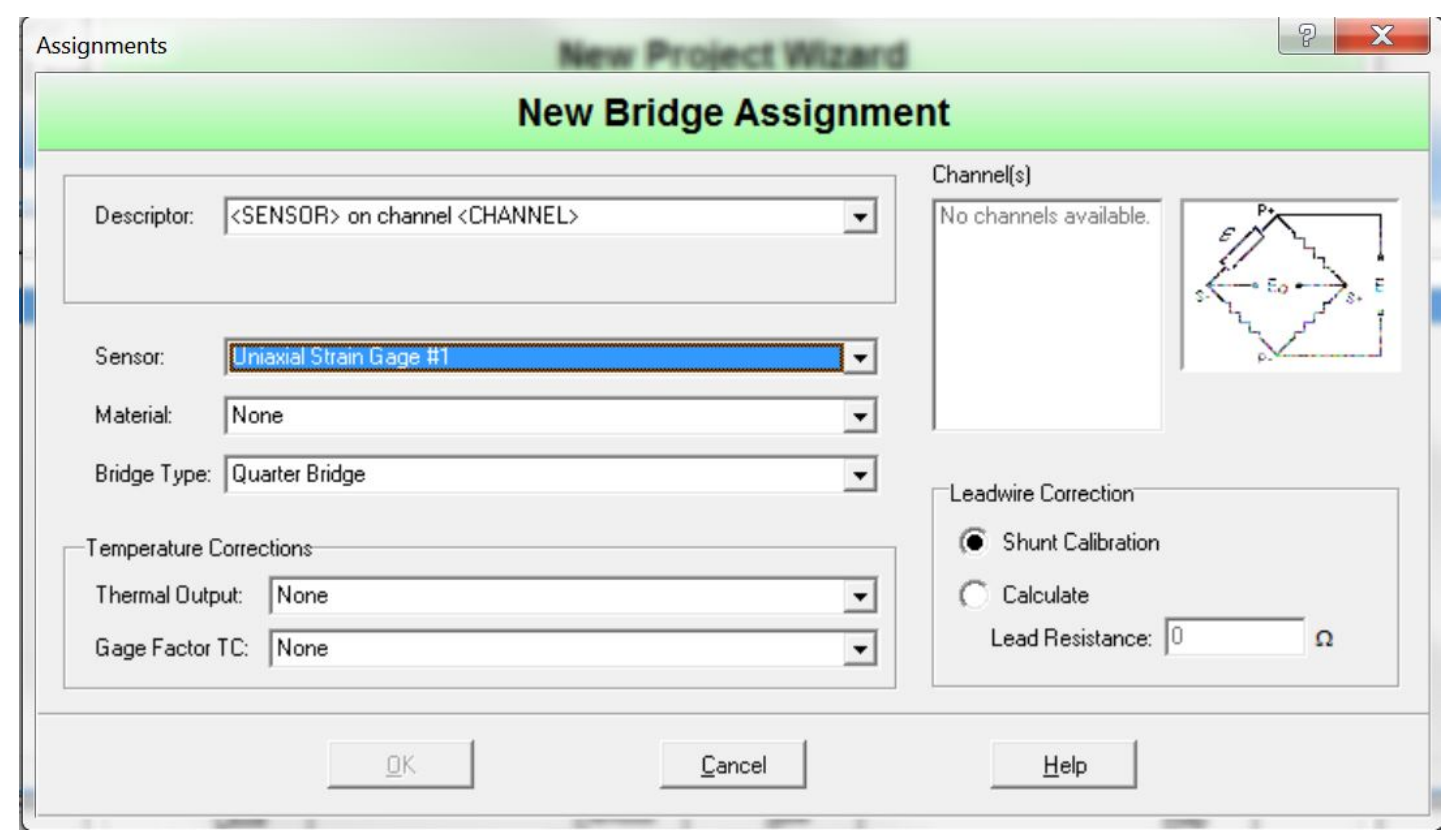

For a high level device do this similarly.

Now you can press next in the wizard window.

16. Simply press finish in the window called "step 6: Organize Assignments (Optional)“"

Save the project where you wish.

17. The new scan button will now be available to be clicked in the main window of the strain smart software. Click on it.

18. The next steps will be to define the scan rate for the data measurements. In the window define the desired number of scans or measurements per second that you would like for the test.

Then press next. 


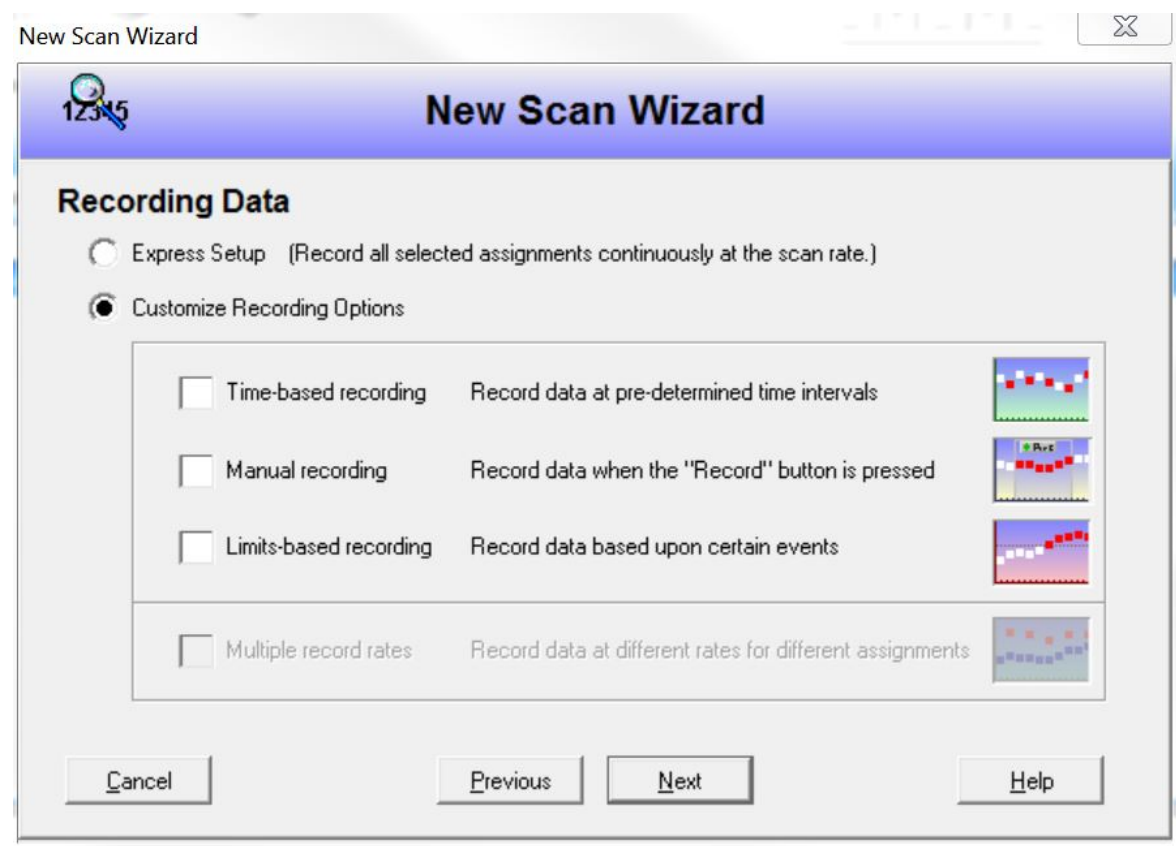

If express setup is selected then the scan rate selected in the previous step will be used continuously.

Selecting the "Customize Recording Options" button allows for other methods for collecting data.

The one that was commonly used in this thesis was manual recording where the user would press the run button to be begin taking data. Then a stop button would be pressed to stop scanning.

Press next.

19. In the next window decide if you would like to collect only a single data point when the button is pressed or if you would like to continuously scan.

The one scan option is useful for static testing and the continuous scanning is good for dynamic or a tensile test.

Then press next twice. 
20. In the Summary window press the finish button.

21. Once this is done make sure that all connections have been made and power one the DAQ.

You may now press the online button in the left bottom corner of the strain smart window.

This will make the daq active and check the connection with the computer.

22. After this the Zero/Cal button will be made available. Click on the zero button. Make sure to zero all channels in the window that pops up.

23. Also make sure to shunt calibrate channels that correspond to a strain gage.

24. After this the daq is ready to be armed by pressing the button called Arm that looks like a traffic signal.

25. The data can be displayed in many forms by clicking on the Display button at top of the window.

26. In the display window that is created you will find small buttons to start and stop data collection and also arm the daq.

27. Once data is collected it will be stored in the software in the "Data" tab as long as a new project is not made.

28. It is advisable to export your results after every test session. This can be 
done by clicking on the scan session in the data tab and selecting the export option.

29. In the windows that appear there are options to output the data collected as text files or files readable in excel.

An important option is to set the number of sig figs that the data would be exported with.

30. After all data is exported open the files and make sure that the data saved correctly.

31. The daq can be powered off at any time once done testing and the wires can all be removed thereafter.
C. Matlab Functions

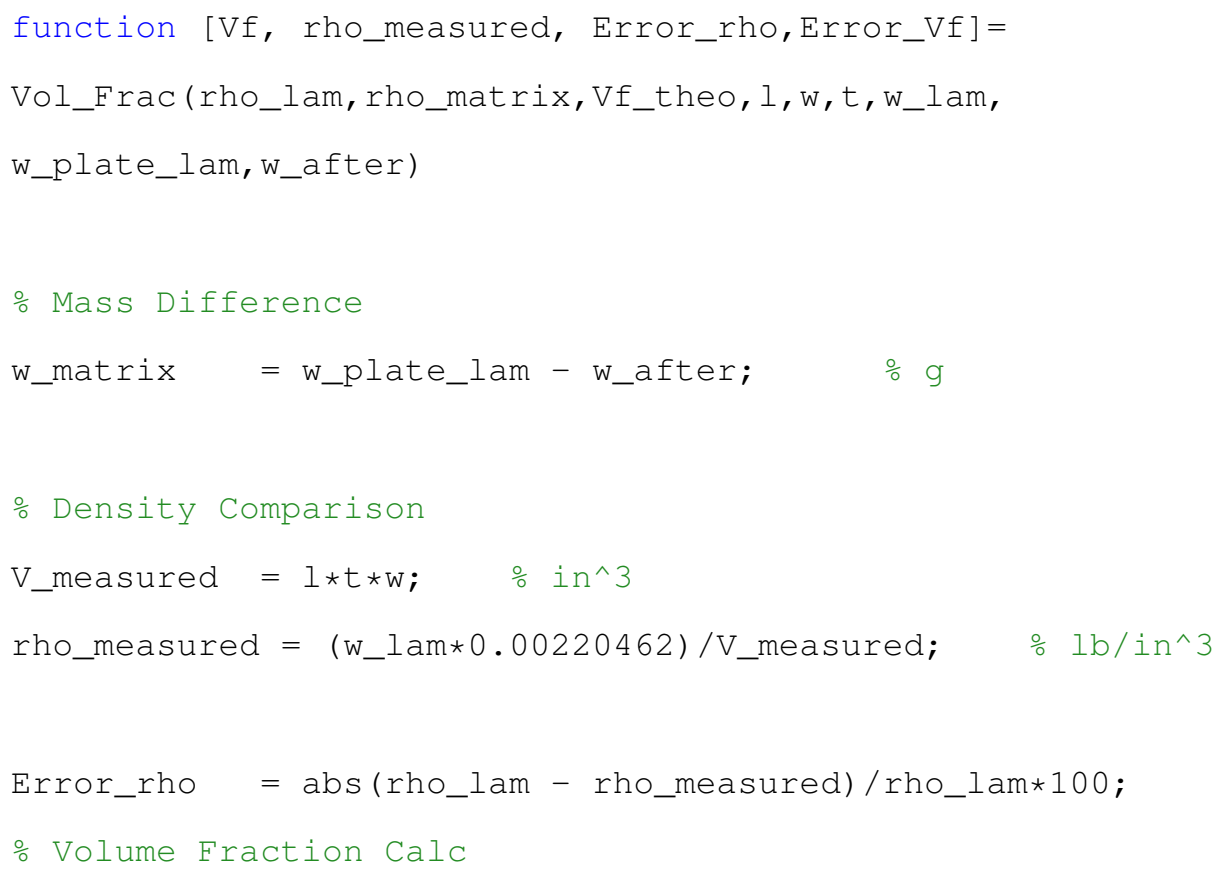




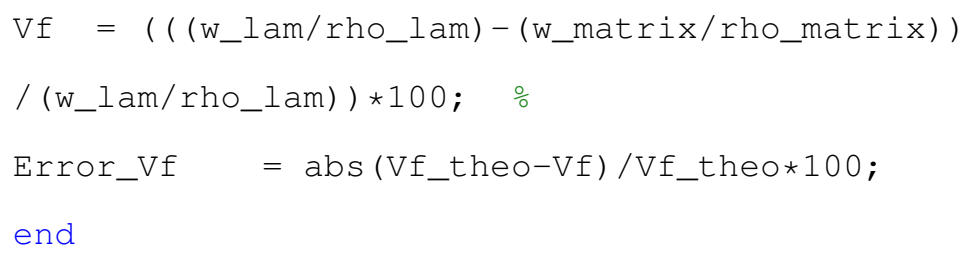

\% Calculation

[Vf_1, rho_measured1, Error_rho1,Error_Vf1] = 


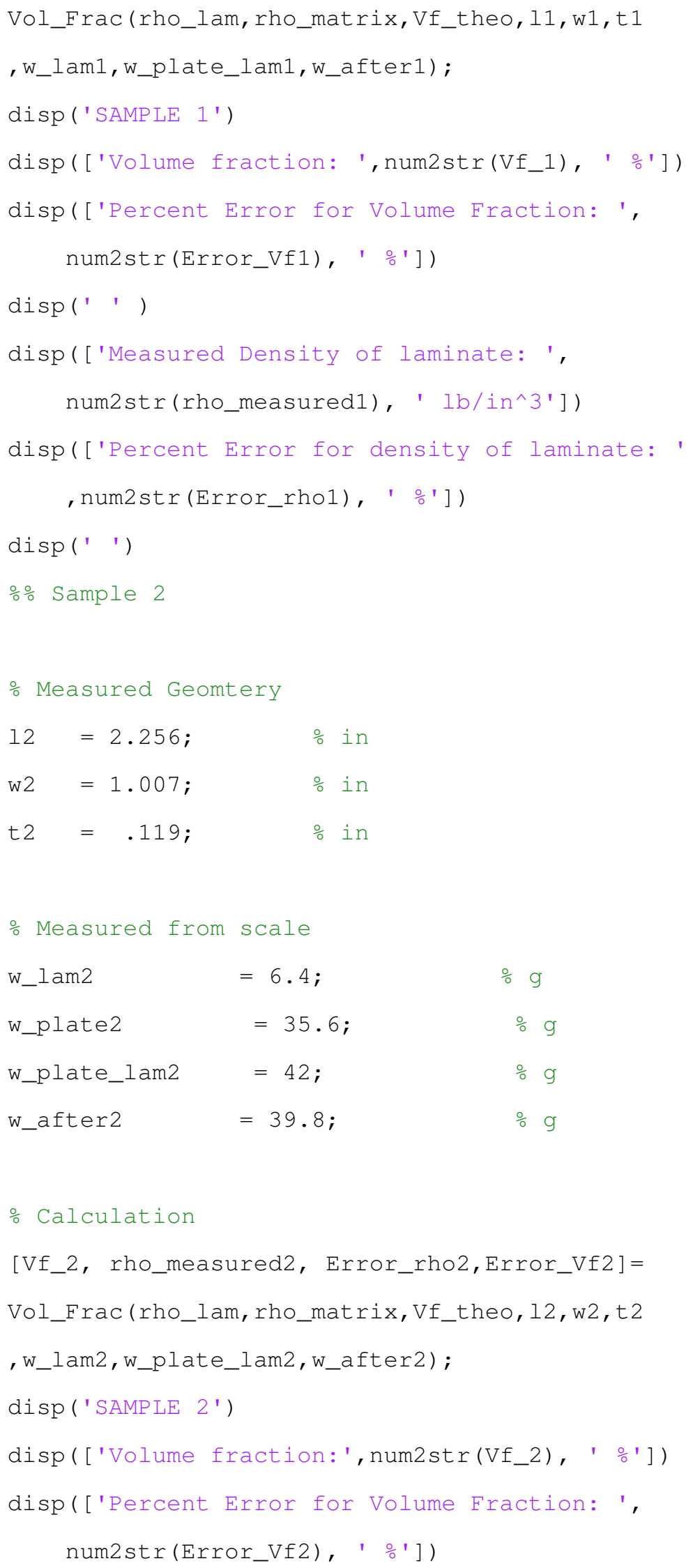




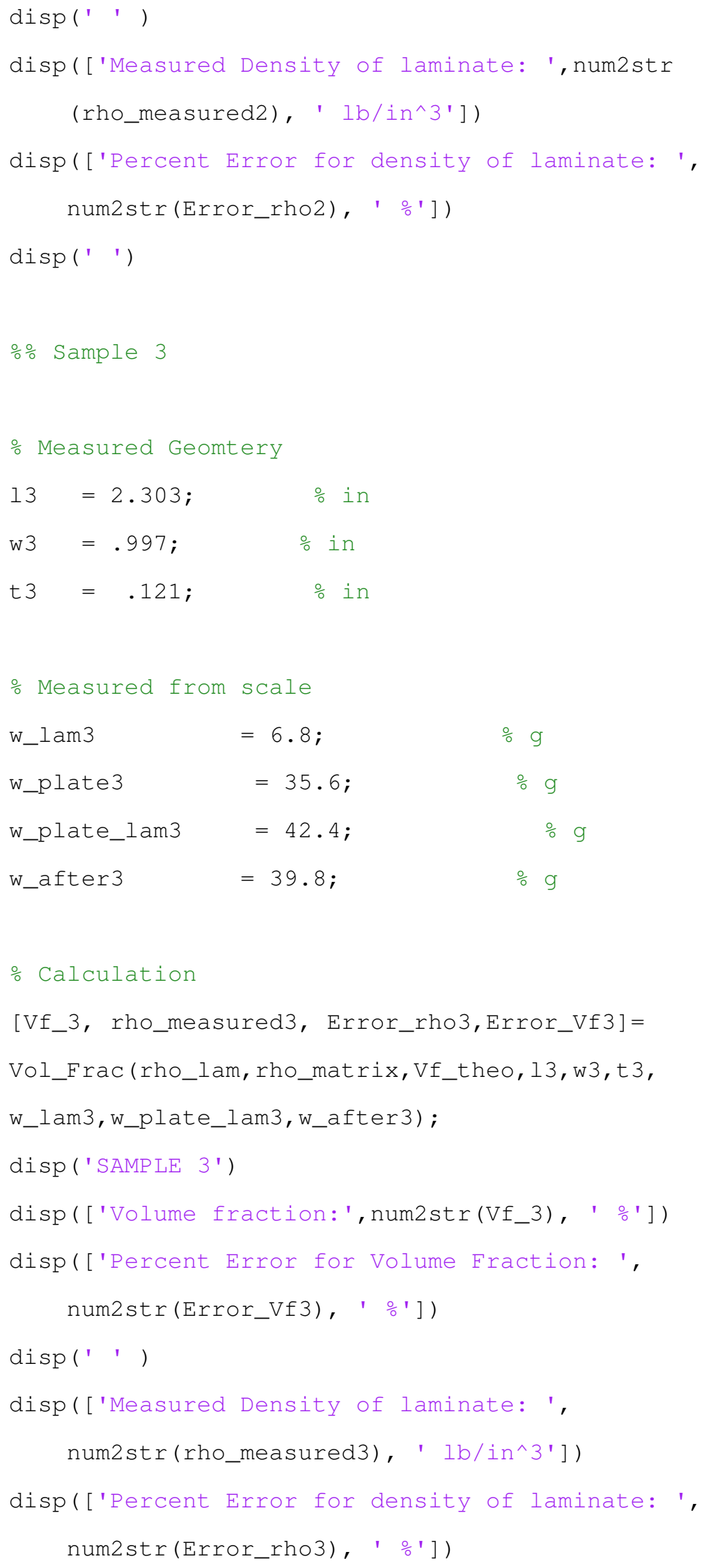




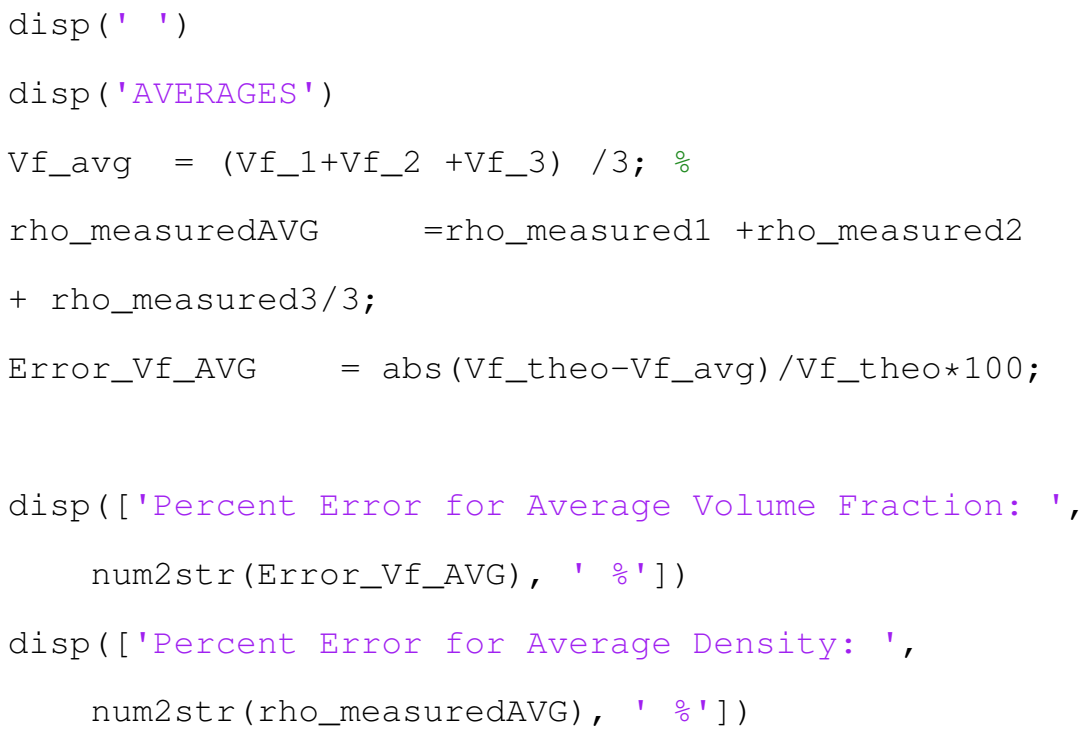


end

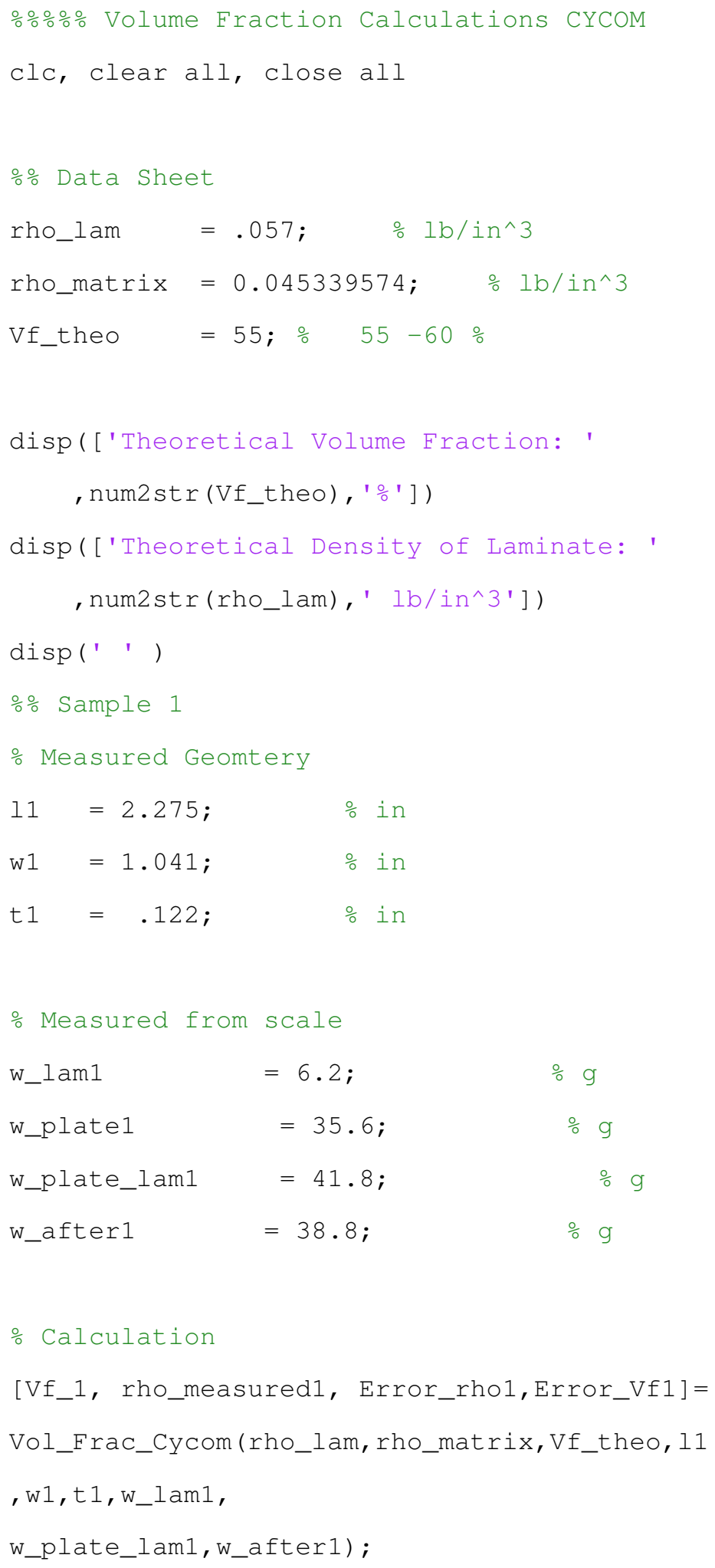




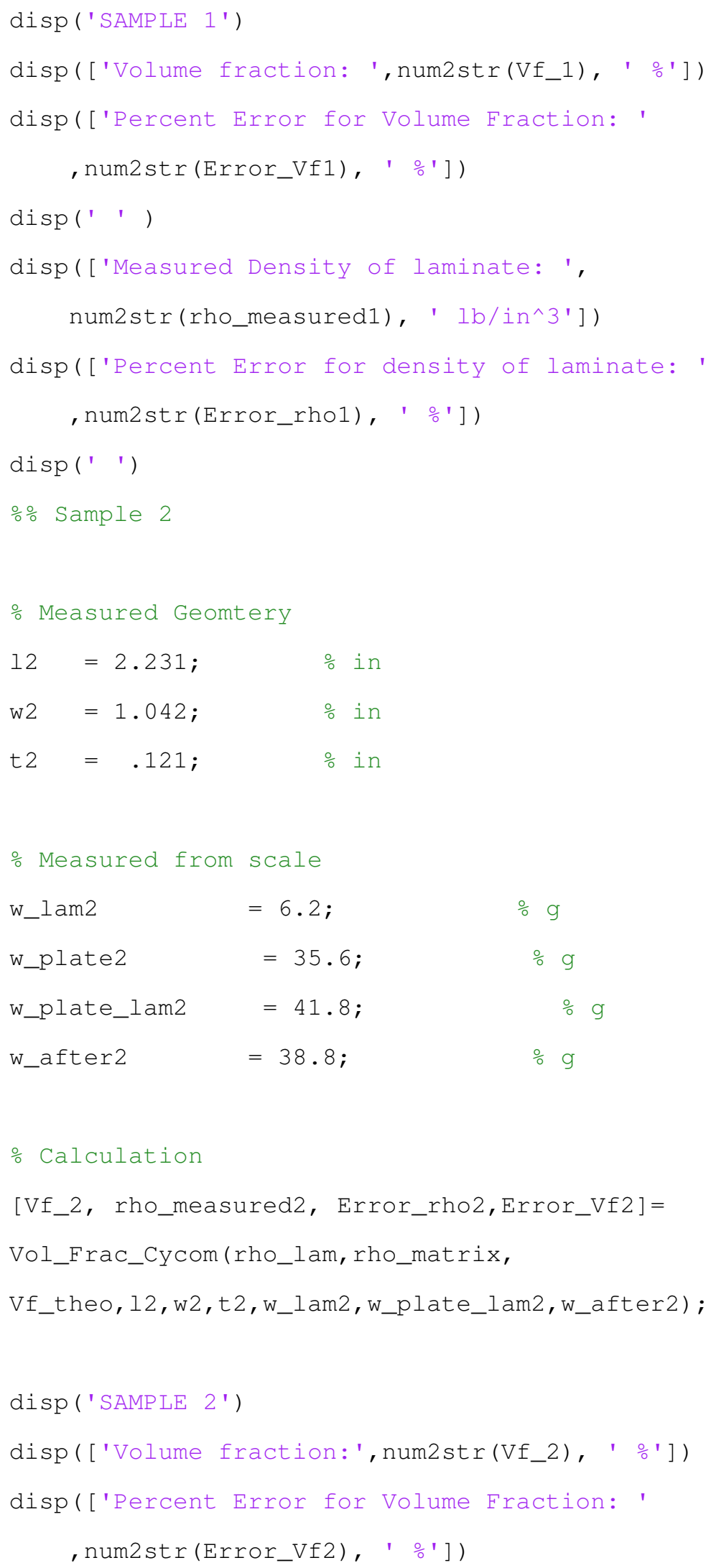




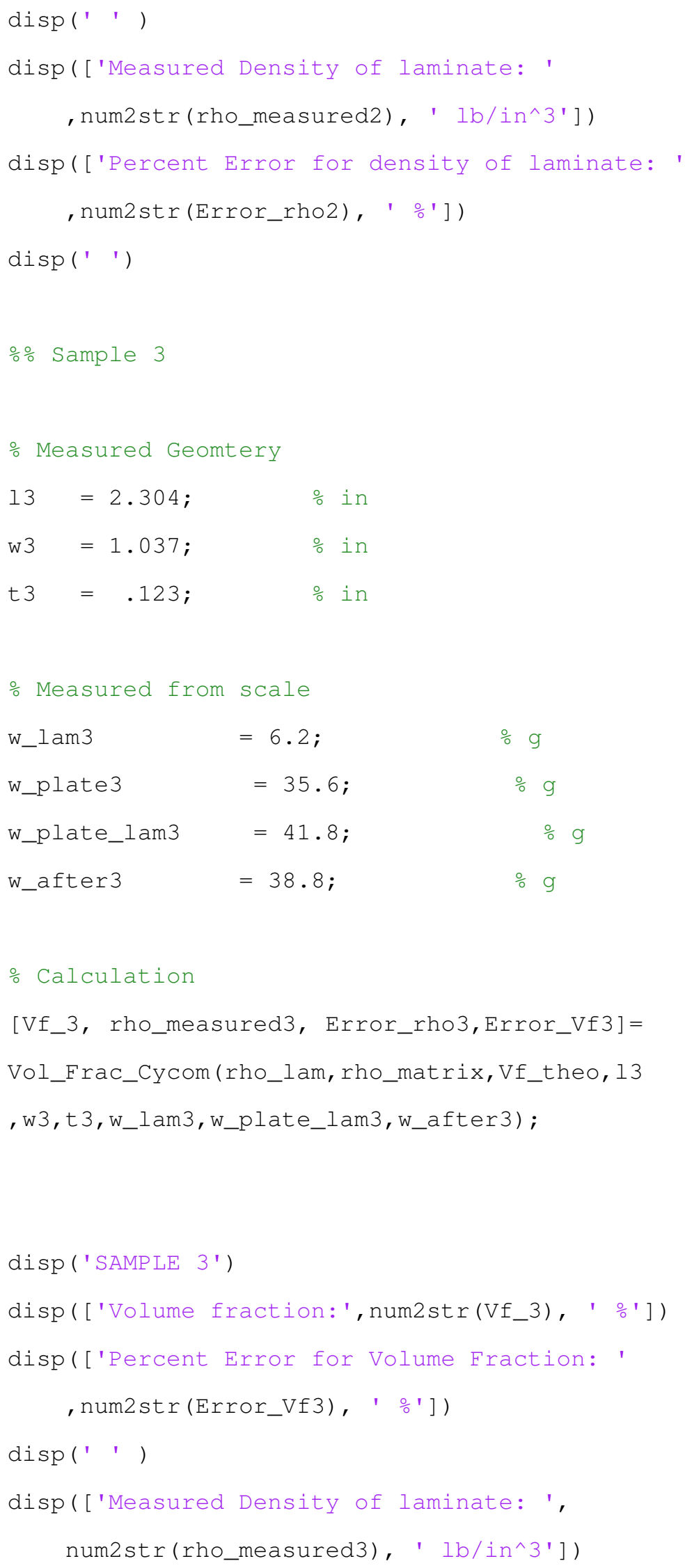




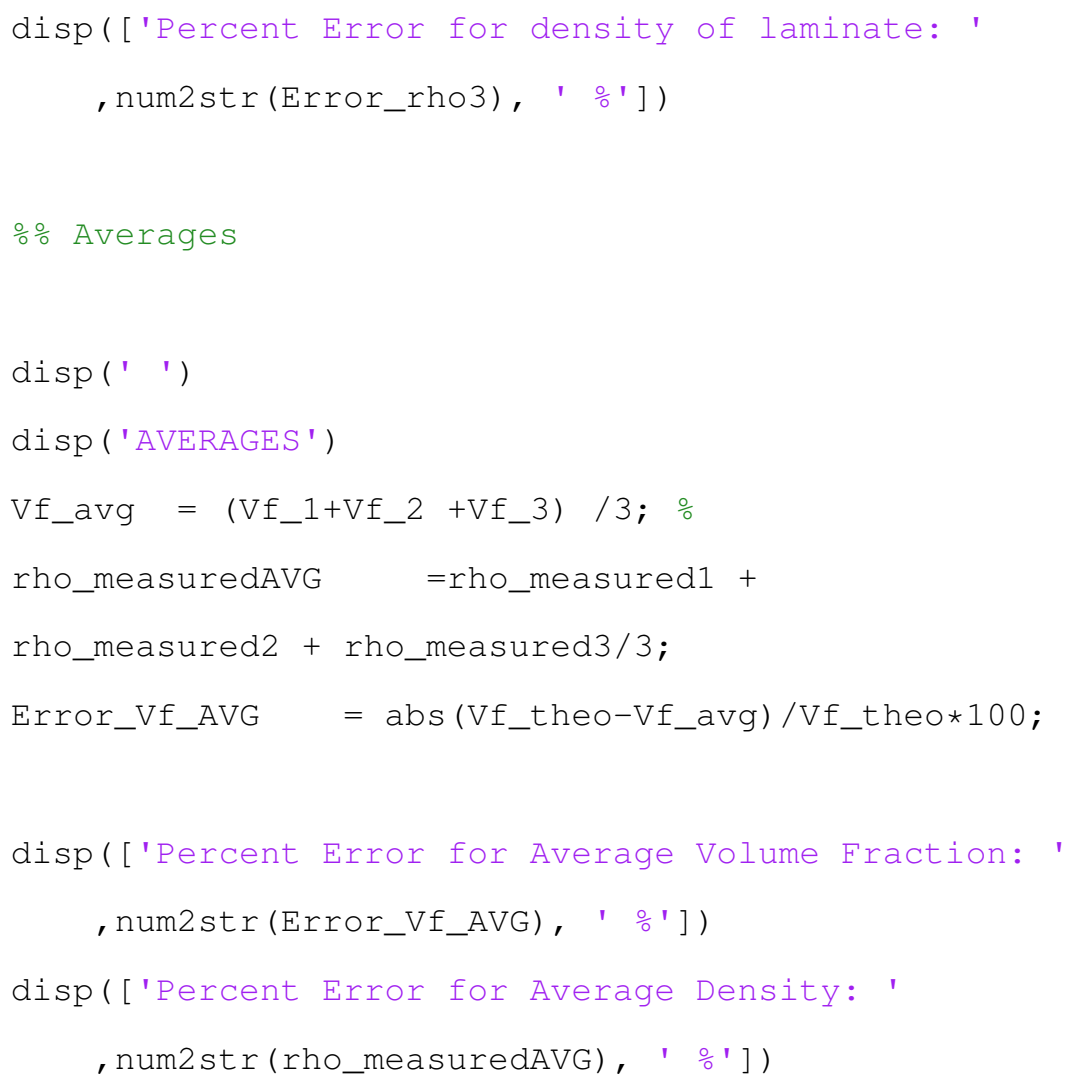

